Kai Bauche

\title{
Segmentierung von Kundendienstleistungen auf investiven Märkten
}


Kai Bauche

\section{Segmentierung von Kundendienstleistungen auf investiven Märkten}

Viele Unternehmen nutzen bestehende Umsatz- und Gewinnmöglichkeiten im Kundendienst bislang nicht konsequent genug aus. Voraussetzung für eine umfassende Ausschöpfung dieser Potentiale ist eine differenzierte und zielgruppengerechte Servicepolitik. Bisher existierten jedoch kaum geeignete Vorschläge zur Segmentierung von Servicemärkten. Die vorliegende Arbeit zeigt praktikable Lösungsmöglichkeiten für dieses kundendienstpolitische Defizit auf. Ausgehend von der servicespezifischen Bedürfnissituation von Unternehmen wird ein zweistufiges Segmentierungsmodell für investive Kundendienstmärkte entwickelt. Auf Grundlage einer empirischen Studie im Markt für kommerzielle Personal Computer werden u.a. praxisrelevante Normstrategien für eine zielgruppengerechte Bearbeitung von Kundendienstmärkten abgeleitet.

Kai Bauche wurde 1961 in Frankfurt a.M. geboren. Studium der Betriebswirtschaftslehre von 1982 bis 1989 an der Universität Münster. Während des Studiums Praktika im In- und Ausland. Zweifach prämierte Diplomarbeit im Bereich Marketing. 1989 Abschluß als Diplom-Kaufmann in Münster. Seither Tätigkeiten im Kundendienstmarketing bei der Nixdorf Computer AG in Paderborn und der Siemens Nixdorf Informationssysteme AG in München. Zwischenzeitliche Beschäftigung am Institut für Marketing der Westfälischen Wilhelms-Universität Münster. 
Segmentierung von Kundendienstleistungen auf investiven Märkten Dargestellt am Beispiel von Personal Computern 


\section{Schriften zu Marketine und Manasement}

Herausgegeben von Prof.Dr. Dr.h.c. Heribert Meffert

Band 23

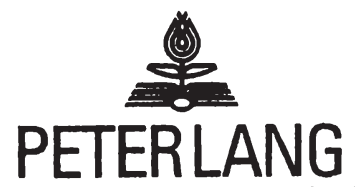

Frankfurtam Main · Berlin · Bern - NewYork · Paris - Wien

Kai Bauche - 978-3-631-75089-6

Downloaded from PubFactory at 01/11/2019 08:30:54AM

via free access 


\section{KaiBauche}

\section{Segmentierung von Kundendienstleistungen aufinvestiven Märkten \\ Dargestelltam Beispielvon Personal Computern}

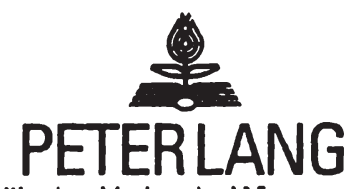

EuropäischerVerlag derWissenschaften

Kai Bauche - 978-3-631-75089-6

Downloaded from PubFactory at 01/11/2019 08:30:54AM 
Die Deutsche Bibliothek - CIP-Einheitsaufnahme

Bauche, Kai:

Segmentierung von Kundendienstleistungen auf investiven Märkten : dargestellt am Beispiel von Personal Computern / Kai Bauche. - Frankfurt am Main ; Berlin ; Bern ; New York ; Paris ; Wien : Lang, 1994

(Schriften zu Marketing und Management ; Bd. 23)

Zugl.: Münster (Westfalen), Univ., Diss., 1993 ISBN 3-631-46808-3

NE: GT

Open Access: The online version of this publication is published on www.peterlang.com and www.econstor.eu under the international Creative Commons License CC-BY 4.0. Learn more on how you can use and share this work: http://creativecommons.org/ licenses/by/4.0.

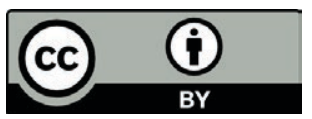

This book is available Open Access thanks to the kind support of ZBW - Leibniz-Informationszentrum Wirtschaft.

\author{
D 6 \\ ISSN 0176-2729 \\ ISBN 3-631-46808-3 \\ ISBN 978-3-631-75089-6 (eBook) \\ (C) Peter Lang GmbH \\ Europäischer Verlag der Wissenschaften \\ Frankfurt am Main 1994 \\ Alle Rechte vorbehalten.
}

Das Werk einschließlich aller seiner Teile ist urheberrechtlich geschützt. Jede Verwertung außerhalb der engen Grenzen des Urheberrechtsgesetzes ist ohne Zustimmung des Verlages unzulässig und strafbar. Das gilt insbesondere für Vervielfältigungen, Übersetzungen, Mikroverfilmungen und die Einspeicherung und Verarbeitung in elektronischen Systemen.

\title{
Printed in Germany 134567
}




\section{Meinen Eltern}

in Dankbarkeit

Kai Bauche - 978-3-631-75089-6

Downloaded from PubFactory at 01/11/2019 08:30:54AM

via free access 
Kai Bauche - 978-3-631-75089-6

Downloaded from PubFactory at 01/11/2019 08:30:54AM

via free access 


\section{Vorwort des Herausgebers}

Die wissenschaftliche Forschung hat kundendienstspezifische Fragestellungen lange Zeit stiefmütterlich behandelt. Aspekte des Servicemarketing wurden erst in den 70 er und zu Beginn der 80 er Jahre verstärkt aufgegriffen. Gegenwärtig erhält diese Diskussion sowohl von wissenschaftlicher als auch von praktischer Seite neue Impulse.

Die Kundendienst- und Servicebereiche bei vielen Unternehmen haben sich inzwischen zu eigenverantwortlichen, umsatz- und gewinnorientierten Geschäftsbereichen entwickelt. Dabei werden vielfach die Wettbewerbsgrenzen neu definiert: Über die unternehmenseigenen Serviceleistungen hinaus wird auch die Betreuung von Konkurrenzprodukten angestrebt. Kundendienst unterliegt damit aber auch zunehmend allen Chancen und Risiken einer eigenständigen Absatzpolitik.

Hierbei kristallisieren sich besonders im Bereich nachfragebedingter Serviceleistungen, den sog. "Kann"-Dienstleistungen, für Kundendienstanbieter in vielen Branchen noch umfangreiche, unausgeschöpfte Wachstumspotentiale heraus. Im Gegensatz zu "Muß"- (bereitschaftsherstellende Dienste) und "Soll"-Leistungen (absehbare Wartungs- und Reparaturdienste), die von den meisten Nachfragern bereits als selbstverständliche "Kulanzleistungen" angesehen werden bzW. im Kaufpreis der Hauptleistung berūcksichtigt werden, hăngen "Kann"-Leistungen von den spezifischen Servicebedürnissen der Verwender ab.

Erst eine attraktive Gestaltung dieser Kundendienste gewährleistet eine wirksame Profilierung gegenüber Konkurrenten und führt zu einer erfolgversprechenden Wettbewerbsposition. Insofern stehen die Anbieter vor der Aufgabe, den Servicebedürfnissen einzelner Marktsegmente mit differenzierten, d.h. zielgruppengerechten Servicelösungen entgegenzutreten.

Obgleich Wissenschaft und Praxis den generellen Stellenwert der Kundendienstpolitik weitestgehend erkannt haben, existieren im Vergleich zum Konsumund Investitionsgütermarketing bislang kaum Vorschlăge zur Segmentierung von Kundendienstmärkten. 
So stellt sich die Aufgabe, bestehende Segmentierungskonzepte hinsichtlich servicebezogener Besonderheiten zu erweitern. Angesprochen sind dabei vor allem die sich aus der Verbundsituation zwischen Hauptleistung und Kundendienst ergebenden Konsequenzen. Aus kundendienstpolitischer Sicht zeigt sich dieser Verbund durch Ausstattungs- und Leistungsmerkmale der Hauptleistung sowie insbesondere durch das kundendienstspezifisches Verwendungsverhalten.

Ferner sind Zeitpunkt und Umfang der Bedürfnisentstehung zu berücksichtigen. Das Verhalten der Kundendienstnachfrager hinsichtlich der Intensität und Art der Inanspruchnahme von Kundendienstleistungen bei Gebrauchsgütern, wie z.B. bei Kraftfahrzeugen und Computern, ändert sich im Lebenszyklus der Produkte. Während Lieferungs- und Installationsleistungen vielfach schon beim Produktkauf vereinbart werden, entsteht eine Nachfrage nach anderen Serviceleistungen, wie z.B. Fahrertrainings von Automobilherstellern und Anwender-Workshops für Computernutzer erst zu einem wesentlich späteren Zeitpunkt der Produktnutzung.

Dieser komplexe Problembereich wird in der vorliegenden Untersuchung aufgegriffen. Auf Grundlage der Erkenntnisse allgemeiner Kundendienstforschung entwickelt der Verfasser am Beispiel von professionell genutzten Personal Computern ein zweistufiges, servicespezifisches Segmentierungsmodell für investive Kundendienstmärkte. In den Mittelpunkt der eigentlichen Marktaufteilung stellt er die kundendienstpolitische Bedürnissituation von Unternehmen. Zur Ableitung genereller Ansatzpunkte der servicespezifischen Marktbearbeitung werden relevante Entscheidungs- und Einflußstrukturen servicebezogener Kaufentscheidungsprozesse von Unternehmen analysiert. Auf der Basis einer Befragung von 198 Unternehmen, die im Rahmen ihrer betrieblichen Datenverarbeitung Personal Computer einsetzen, erfolgt eine fundierte Abgrenzung verschiedener Kundendienstsegmente.

Dem Verfasser ist mit seiner Arbeit die Synthese zwischen theoretischer Fundierung und empirischer Überprüfung praxisrelevanter Fragestellungen des Kundendienstmarketing gut gelungen. Vor dem Hintergrund des bislang bestehenden Forschungsdefizits auf dem Gebiet der Kundendienstsegmentierung zeigt die Untersuchung interessante weiterführende Ansatzpunkte für eine differenzierte Kundendienstpolitik auf. Es ist daher zu wünschen, daß sie in Wissenschaft und Praxis entsprechende Beachtung erfährt. 
Die Erhebung der umfangreichen Datenbasis der vorliegenden Untersuchung erfolgte mit Unterstützung der Siemens Nixdorf Informationssysteme AG in München. Dafür gilt mein besonderer Dank.

Münster, im August 1993

Prof. Dr. Dr. h.c. Heribert Meffert 
Kai Bauche - 978-3-631-75089-6

Downloaded from PubFactory at 01/11/2019 08:30:54AM

via free access 


\section{Vorwort des Verfassers}

Die Betriebswirtschaftslehre setzt sich seit geraumer Zeit intensiv mit Aspekten der Kundendienstpolitik auseinander. Dabei hat sich der Service in vielen Branchen mittlerweile zu einem zentralen Umsatz- und Gewinnträger entwickelt. An Aktualität gewinnt diese Diskussion angesichts der gegenwärtig rezessiven Tendenzen auch aufgrund des nachhaltigen Einflusses der Servicepolitik in bezug auf das Kaufentscheidungsverhalten der Nachfrager, die Kundenzufriedenheit und damit nicht zuletzt auch auf die Kundenbindung selbst. Vor diesem Hintergrund erlangt eine differenzierte Kundendienstpolitik besondere Bedeutung. Dies setzt jedoch eine spezifische Marktsegmentierung voraus. Ein direkter Transfer bestehender Hauptleistungskonzepte zur Marktaufteilung und -bearbeitung kann diesen Anforderungen nur unzureichend gerecht werden.

Mit der vorliegenden Arbeit wird das Ziel verfolgt, ein spezifisches Segmentierungsmodell für investive Kundendienstmärkte zu entwickeln, um bedürfnishomogene Gruppen von Servicenachfragern identifizieren zu können. Darüber hinaus soll die Machtsituation einzelner Buying Center-Mitglieder beim Leistungskauf analysiert werden, um grundlegende Marktbearbeitungsstrategien i.S.v. Normstrategien für den Service ableiten zu können. Die Identifikation trennscharfer Segmente im Kundendienstmarkt für kommerzielle Personal Computer (PCs) stellt dabei die empirische Zielsetzung der Arbeit dar.

Angesichts der bisher noch bestehenden Forschungslücke zur Segmentierung von Servicemärkten versteht sich die Arbeit letztlich als Basisuntersuchung. Neben der Beantwortung der zugrundeliegenden Untersuchungshypothesen, sollen insbesondere Ansatzpunkte für weiterführende Fragestellungen bereitgestellt werden.

Die Abfassung der Arbeit war nur mit vielfältiger Unterstützung verschiedener Personen möglich.

Mein ganz besonderer Dank gilt an dieser Stelle meinem akademischen Lehrer Herrn Prof. Dr. Dr. h.c. Heribert Meffert. Umfassend förderte und begleitete er die Fertigstellung dieser Arbeit in allen Phasen. Herrn Prof. Dr. Klaus Backhaus sei für die Übernahme des Zweitgutachtens herzlich gedankt. 
Die der Arbeit zugrunde liegende empirische Untersuchung wurde von mir im Rahmen eines Forschungsprojektes der Siemens Nixdorf Informationssysteme AG durchgeführt. SNI unterstützte das Projekt, aber auch mich persönlich in vorbildlicher Weise und dokumentierte damit ein beispielgebendes Zusammenspiel von Wissenschaft und Praxis. Stellvertretend für die vielfältige Unterstützung und Hilfsbereitschaft, die mir entgegengebracht wurde, gilt mein Dank Herrn Dipl.- Ing. Peter Burgdorf, Herrn Dipl.- Ing. Dipl.- Wirt.- .Ing. Karl Abril und Herrn Dipl.- Ing. Karl Kuntke. Ganz besonders möchte ich mich aber bei Herrn Dipl.- Kfm. Guido Kaufmann bedanken und ihm an dieser Stelle viel Erfolg für seine eigene Forschungsarbeit wünschen.

Nicht zuletzt gilt mein Dank allen Kolleginnen und Kollegen am Institut für Marketing. Im Mittelpunkt steht dabei Herr Dipl.- Kfm. Dr. Marcus Poggenpohl. Selbstlos hat er an vielen Wochenenden und in ungezählten nächtlichen Diskussionsrunden zum Gelingen dieser Arbeit beigetragen. Ein herzlicher Dank gilt auch Herrn Prof. Dr. Martin Benkenstein, von dessen konstruktiven Verbesserungsvorschlägen ich sehr profitiert habe. Bei Herrn Dipl.- Kfm. Dr. Manfred Kirchgeorg bedanke ich mich für die vielfältigen Diskussionen und Anregungen.

Meinen Eltern gebührt ein besonders tiefer Dank. Ohne ihre Liebe und Hilfe hätte ich dieses Ziel nicht erreichen können. Sie haben mich stets in umfassender, selbstloser Weise unterstützt und mir dabei stets Vertrauen entgegen gebracht. Schließlich aber möchte ich ganz besonders meiner Verlobten Julia Vorwerk danken. Ohne ihr Engagement, ihren Zuspruch und ihre tatkräftige Hilfe hätte ich diese Arbeit nicht schreiben können. 


\section{INHALTSVERZEICHNIS}

\section{A. MARKTSEGMENTIERUNG VON KUNDENDIENSTLEISTUNGEN ALS} HERAUSFORDERUNG DES INVESTITIONSGÜTERMARKETING

1. Entwicklung und Bedeutung des Kundendienstes im Wandel der Absatzpolitik.

2. Kundendienst als Gegenstand der Marktsegmentierung .5

2.1 Kundendienst als Spezialfall des investiven Dienstleistungsmarketing 5

2.2 Kundendienstleistungen als eigenständige Absatzleistungen. 10

2.3 Marktsegmentierung von Kundendienstleistungen 15

3. Der Markt für kommerzielle Personal Computer als Gegenstand der Untersuchung 19

4. Ziel und Gang der Untersuchung 23

B. KONZEPTIONELLE GRUNDLAGEN ZUR SEGMENTIERUNG VON KUNDENDIENSTLEISTUNGEN IN INVESTIVEN MÄRKTEN.

1. Ansätze der Marktsegmentierung und ihre Übertragbarkeit auf Kundendienstprobleme

1.1 Marktsegmentierung im Spannungsfeld der Marketingforschung...... 28

1.2 Mehrstufige Segmentierungsansätze. 33

1.3 Bewertung bestehender Segmentierungsansätze in bezug auf investive Kundendienstleistungen

2. Die Operationalisierung des Kundendienstbedarfs von Unternehmen

2.1 Brückenvariablen als Mittel zur Operationalisierung des Kundendienstbedarfs.

2.2 Kundendienstbezogene Brückenvariablen

2.21 Der hauptleistungsbezogene Abhängigkeitsgrad. 47

2.22 Die hauptleistungsbezogene Kundenkompetenz 51 
2.3 Kundendienstbezogene Ausgangsvariablen.................................. 57

2.31 Variablen der Makrosegmentierung ...................................... 57

2.32 Variablen der Prozeß- und Strukturanalyse ............................... 60

2.33 Variablen der Mikrosegmentierung ......................................... 62

3. Kundendienstpolitische Marktsegmentierung als zweistufiges Problem

3.1 Die Bildung bedarfsorientierter Kundendienstsegmente als primäre Problemstufe

3.2 Marktbearbeitungsorientierte Analyse kaufrelevanter Machtstrukturen als sekundäre Problemstufe 68

4. Bezugsrahmen und Hypothesen der Untersuchung 70

C. EMPIRISCHE ANALYSE ZUR SEGMENTIERUNG VON KUNDENDIENSTLEISTUNGEN IN INVESTIVEN MÄRKTEN 73

1. Design der Untersuchung 73

2. Zentrale Voraussetzungen der Kundendienstsegmentierung..... 76

2.1 Die Einbindung der EDV-Funktion in die Organisation von Unternehmen

2.2 Der EDV-Beauftragte als Kompetenzführer bei PC-bezogenen Aufgabenstellungen 78

3. Die Bildung bedarfsorientierter Kundendienstsegmente im Markt für kommerzielle Personal Computer

3.1 Die Bildung kundendienstbezogener Kerndimensionen 83

3.11 Der Abhängigkeitsgrad 83

3.12 Die Kundenkompetenz. 88

3.2 Kundendienstsegmente des Marktes für kommerzielle Personal Computer 
3.21 Methodische Grundlagen zur Gruppenbildung........................ 93

3.22 Darstellung bedürnisbezogener Kundendienstsegmente.......... 97

3.221 Das kundendienstunsensible Segment......................... 98

3.222 Das Kundendienst-Nischensegment ............................. 102

3.223 Das Standard-Kundendienstsegment............................. 105

3.224 Das kundendienstskeptische Segment.......................... 108

3.225 Das kundendienstunbetroffene Segment ....................... 112

3.226 Das kundendienstignorante Segment.............................115

3.23 Trennschärfe der Kundendienstsegmente ................................ 119

4. Die Analyse segmentspezifischer Machtstrukturen bei kundendienstbezogenen Kaufentscheidungsprozessen ................................................ 123

4.1 Machtpromotoren bei Kundendienstkaufsituationen............................ 124

4.2 Fachpromotoren bei Kundendienstkaufsituationen ........................... 128

4.3 Initiatoren kundendienstbezogener Kaufentscheidungsprozesse....... 133

5. Beurteilung der empirischen Segmentierungsergebnisse ........................... 138

D. IMPLIKATIONEN DER UNTERSUCHUNG .............................................. 145

1. Marktbearbeitungsstrategische Implikationen .......................................... 145

1.1 Formen der Integration von Personal Computern in den Prozeß betrieblicher Datenverarbeitung...................................................... 146

1.2 Entwicklung kundendienstpolitischer Normstrategien ........................ 150

2. Ansatzpunkte für die weitere Forschung ................................................. 155

Anhang l: Tabellen der empirischen Untersuchung ............................................. 159

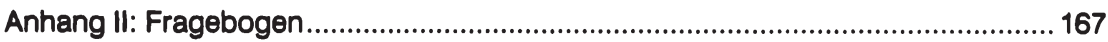

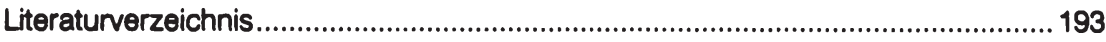


Kai Bauche - 978-3-631-75089-6

Downloaded from PubFactory at 01/11/2019 08:30:54AM

via free access 


\section{ABBILDUNGSVERZEICHNIS}

Abbildung 1: Systematisierung von Dienstleistungsarten ............................ 6

Abbildung 2: Typologie von Kundendienstleistungen am Beispiel von Computerservices ........................................................ 12

Abbildung 3: Zuordnung von Serviceleistungen zu verschiedenen Kaufentscheidungssituationen .............................................. 14

Abbildung 4: Segmentierungsproblematik am Beispiel des Computermarktes....................................................................... 18

Abbildung 5: Basissegmente des Computermarktes ................................ 21

Abbildung 6: Kundendienstbezogene Nutzensituation................................ 27

Abbildung 7: Ablaufschema der zweistufigen Marktsegmentierung nach Wind/Cardozo

Abbildung 8: Aggregationsproblematik des Segmentierungsansatzes von Wind/Cardozo .................................................. 38

Abbildung 9: Analyse der Verhaltensrelevanz nach Gröne.......................... 41

Abbildung 10: Konstrukt der Verhaltensrelevanz von Segmentierungskriterien.......................................................................... 46

Abbildung 11: Integrationsformen von Personal Computern ........................ 50

Abbildung 12: PC-bezogene Kompetenzstrukturen ..................................... 56

Abbildung 13: Strukturvergleich investitionsgut- und kundendienstbedürfnisbezogener Segmentierungsprozesse..................... 63

Abbildung 14: Bedarfsniveau für Kundendienstleistungen ............................ 64

Abbildung 15: Zusammenhang zwischen Ausgangs- und Brückenvariablen im Rahmen der Bildung bedūrfnisbezogener Kundendienstsegmente

Abbildung 16: Bezugsrahmen der empirischen Untersuchung.................... 71

Abbildung 17: Institutionalisierung der EDV-Funktion .................................. 77 
Abbildung 18: PC-Kompetenz verschiedener Funktionsträger.................. 80

Abbildung 19: PC-bezogene Gruppenkompetenz ................................ 82

Abbildung 20: PC-Abhängigkeit in verschiedenen Unternehmensbereichen

Abbildung 21: Varianzkriterium für die Clusterlösung............................... 96

Abbildung 22: Besetzung der 6-Cluster-Lösung .................................... 98

Abbildung 23: Kundendienstbezogene Bedeutungs- und Zufriedenheitsbeurteilung des kundendienstunsensiblen Segments.

Abbildung 24: Kundendienstbezogene Bedeutungs- und Zufriedenheitsbeurteilung des Kundendienst-Nischensegments

Abbildung 25: Kundendienstbezogene Bedeutungs- und Zufriedenheitsbeurteilung des Standard-Kundendienstsegments.

Abbildung 26: Kundendienstbezogene Bedeutungs- und Zufriedenheitsbeurteilung des kundendienstskeptischen Segments.

Abbildung 27: Kundendienstbezogene Bedeutungs- und Zufriedenheitsbeurteilung des kundendienstunbetroffenen Segments.

Abbildung 28: Kundendienstbezogene Bedeutungs- und Zufriedenheitsbeurteilung des kundendienstignoranten Segments.

Abbildung 29: Gütemaße der Diskriminanzfunktion (SPSS-Tableau) ........... 120

Abbildung 30: Entscheidungsmacht bei PC- und kundendienstbezogenen Kaufentscheidungen

Abbildung 31a: Segmentspezifische Entscheidungsmacht bei PCund kundendienstbezogenen Kaufsituationen (Segmente 1, 2 und 3) 
Abbildung 31b: Segmentspezifische Entscheidungsmacht bei PCund kundendienstbezogenen Kaufsituationen (Segmente 4, 5 und 6)

Abbildung 32: Entscheidungseinfluß bei PC- und kundendienstbezogenen Kaufentscheidungen

Abbildung 33a: Segmentspezifische Einflußstrukturen bei PC- und kundendienstbezogenen Kaufentscheidungsprozessen (Segmente 1, 2 und 3) 130

Abbildung 33b: Segmentspezifische Entscheidungsmacht bei PCund kundendienstbezogenen Kaufentscheidungsprozessen (Segmente 4, 5 und 6) 131

Abbildung 34: Anregungsverhalten zu PC- und kundendienstbezogenen Kaufentscheidungsprozessen 133

Abbildung 35a: Segmentspezifisches Anregungsverhalten von PCund kundendienstbezogenen Kaufentscheidungsprozessen (Segmente 1, 2 und 3) 135

Abbildung 35b: Segmentspezifisches Anregungsverhalten von PCund kundendienstbezogenen Kaufentscheidungsprozessen (Segmente 4, 5 und 6)

Abbildung 36: Gegenwärtiger und zukünftiger Stellenwert von Personal Computern im Rahmen der unternehmensbezogenen Datenverarbeitung

Abbildung 37: Gegenwärtige und geplante PC-Konfigurationen von Unternehmen

Abbildung 38: Strategien zur Implementierung von Personal Computern in betriebliche Datenverarbeitungprozesse 148

Abbildung 39: Normstrategien der Kundendienstpolitik 151 


\section{TABELLENVERZEICHNIS}

Tabelle 1: Überblick über ausgewählte Auffassungsinhalte der Marktsegmentierung.

Tabelle 2: Faktoranalytisch verdichtete Dimensionen des Abhängigkeitsgrades von Personal Computern

Tabelle 3: Faktoranalytisch verdichtete Dimensionen der PC-Kompetenz von Unternehmen 90

Tabelle 4: Input-Faktoren der kundendienstbezogenen Clusteranalyse.

Tabelle 5: Diskriminatorische Bedeutung segmentbildender Faktoren.

Tabelle 6: Rollensituation in kundendienstbezogenen Kaufentscheidungsprozessen

Tabelle 7: Zusammenfassende Übersicht zur Prüfung der Untersuchungshypothesen. 


\section{ABKÜRZUNGSVERZEICHNIS}

a.a.O.

Abb.

AL

ASM

asw

Aufl.

AW

AWSW

b.

Bd.

BGB

BS

bzgl.

ca.

Cal.

CORR

DBW

d.h.

DF

Diss.

DOS

DV

EDV

EDV-B

ed.

et. al.

etc.

f. am angegebenen Ort

Abbildung

Abteilungsleiter

Aktueller Software Markt

Absatzwirtschaft

Auflage

Anwender

Anwendersoftware

bei

Band

Bürgerliches Gesetzbuch

Betriebssystem

bezüglich

circa

California

Correlation

Die Betriebswirtschaft

das heißt

Degrees of Freedom

Dissertation

Disk Operating System

Datenverarbeitung

elektronische Datenverarbeitung

EDV-Beauftragter

Edition

et alteri

et cetera

folgende 
f.d.

ff.

FCN

FQS

Frankfurt/M.

GF

GS

HBR

Hrsg.

HW

HWA

i.d.R.

incl.

i.e.S.

insb.

i.R.

i.S.e.

i.S.v.

Jg.

JMR

JoM

Kap.

KD

$\mathrm{kfm}$.

Ifd.

MA

Mass.

MS

N.J.

$\mathrm{Nr}$.

No. für den

fortfolgende

Function

Fehlerquadratsumme

Frankfurt am Main

Geschäftsführer

Grundservice

Harvard Business Review

Herausgeber

Hardware

Handwörterbuch der Absatzwirtschaft

in der Regel

inclusive

im engeren Sinne

insbesondere

im Rahmen

im Sinne einer

im Sinne von

Jahrgang

Journal of Marketing Research

Journal of Marketing

Kapitel

Kundendienst

kaufmännisch

laufend

Mitarbeiter

Massachusetts

Microsoft

New Jersey

Nummer

Number 
0.0.

ohne Ortsangabe

OS/2

Operating System 2

o.V.

ohne Verfasser

$\mathrm{PC}(\mathrm{s})$

Personal Computer (Plural)

PCT

Percent

S.

Seite

SIG

Signifikanz

sog.

sogenannte

SPSS

Statistical Package for Social Sciences

SW

Software

Tab.

Tabelle

TKD

Technischer Kundendienst

u.a.

und andere

u.U.

unter Umständen

vgl.

vergleiche

Vol.

Volume

z.B.

zum Beispiel

$\mathrm{Zfb}$

Zeitschrift für Betriebswirtschaft

ZfbF

Zeitschrift für betriebswirtschaftliche Forschung

ZFP

Zeitschrift für Forschung und Praxis 
Kai Bauche - 978-3-631-75089-6

Downloaded from PubFactory at 01/11/2019 08:30:54AM

via free access 


\section{A. Marktsegmentierung von Kundendienstleistungen als Herausforde- rung des Investitionsgütermarketing}

\section{Entwicklung und Bedeutung des Kundendienstes im Wandel der Ab- satzpolitik}

Kundendienstleistungen gewinnen seit geraumer Zeit sowohl für Hersteller als auch für Kunden an Bedeutung. Zentrale Ursachen für diesen Wandel liegen in einer fortschreitend technologiebestimmten Lebens- und Arbeitswelt, ansteigender Technologiekomplexität und einem dadurch bedingten zunehmenden Servicebewußtsein der Nachfrager. ${ }^{1}$ Darüber hinaus hat die fortschreitende Ähnlichkeit der Leistungsangebote in vielen Branchen dazu geführt, daß sich die Hersteller gegenüber ihren Wettbewerbern häufig nur noch durch spezifische Dienstleistungsangebote differenzieren und damit komparative Konkurrenzvorteile aufbauen können. ${ }^{2}$ Diese Entwicklungen treffen in besonderem Maße auf technologiedominante Produkte zu. In der Haushaltsgeräte-, HiFi-, Fernseh-, Film-/Foto-, Freizeit- und insbesondere der elektronischen Datenverarbeitungsindustrie spielen Umfang und Qualität des Kundendienstes häufig eine zentrale Rolle im Kaufentscheidungsprozeß der Nachfrager. ${ }^{3}$

Institutionelle Nachfrager stehen in diesem Zusammenhang vor einer besonderen Herausforderung. Einerseits besteht die Notwendigkeit, stets modernste Informations-, Kommunikations- und Datenverarbeitungstechnologien in ihren Unternehmen einzusetzen, um Wettbewerbsvorteile auf- und auszubauen. 4

Andererseits erschweren die ansteigende technologische Komplexität, unverständliche Bedienungsanleitungen sowie mangelnde Informationen über Nutzungsmöglichkeiten jedoch vielfach die "Beherrschbarkeit" hochtechnisierter Systeme. Es kommt zu wachsenden Unsicherheiten, längeren Einarbeitungszeiten und zunehmendem Hardware- und Softwareunverständnis der Anwender. Be-

1 Vgl. Meffert, H., Kundendienstpolitik, in: Marketing ZFP, 9. Jg., 1987, Nr. 2, S. 93.

2 Nach Backhaus besitzt ein Unternehmen dann einen komparativen Konkurrenzvorteil, "...wenn es in der Wahrnehmung seiner Abnehmer ein besseres Leistungsangebot im Hinblick auf das nachfragerbezogene Problemlösungspotential besitzt als die Konkurrenz."; Backhaus, K., Investitionsgütermarketing, 2. Auff., München 1990, S. 6 f.

3 Vgl. Little, A.D., Innovation als Führungsaufgabe, Frankfurt/M., New York 1988, S. 148 f.

4 Vgl. Porter, M.E., Wettbewerbsvorteile, Frankfurt/M., New York 1986, S. 223. 
dienungsfehler und damit verbundene Motivationseinbußen und Produktivitätsverluste sind die Folge. Wettbewerbsvorteile, die Investoren mit dem Kauf solcher Technologien anstreben, gehen so häufig wieder verloren. 5

Aus diesen Gründen wächst der Bedarf an umfassender, kompetenter Anwendungsunterstützung, um die Funktionalität und den Nutzen derartiger Technologien sicherzustellen. 6 Diese umfaßt neben der technischen Hilfestellung insbesondere die Beratung der Systemanwender. In der Konsequenz ergibt sich damit ein zusätzlicher Kundendienstbedarf von Nachfragern.

Auch die Anbieter haben den veränderten Stellenwert von Kundendienstleistungen erkannt. Der Einfluß von Service auf das Kaufentscheidungsverhalten der Nachfrager, und nicht zuletzt die Möglichkeit durch Dienstleistungen das Produkt während der gesamten Lebensdauer beim Kunden zu begleiten, haben dazu beigetragen. Hierbei steht vor allem die besondere Bedeutung von Services in bezug auf psychographische Zielgrößen wie Kundenzufriedenheit, Kundenbindung und Präferenzbildung im Vordergrund. Letztendlich beeinflussen diese auch ökonomische Erfolgsgrößen wie Umsatz und Gewinn. ${ }^{7}$ Die Auswirkungen, die sich vor allem in bezug auf die Produktzufriedenheit des Kunden ergeben sind gravierend. Die hieraus resultierende Kompetenzbeurteilung des Anbieters und der ihm entsprechend beigemessene Vertrauensvorsprung haben entscheidenden Einfluß auf die Wiederkaufchancen eines Produktes ("Die erste Maschine verkauft der Vertrieb, die zweite verkauft der Kundendienst")8.

In vielen Branchen hat sich deshalb das kundendienstbezogene Leistungsangebot über traditionelle Reparatur- und Wartungsdienste hinaus um umfassende, an den Kundenbedürfnissen orientierte Serviceleistungen erweitert. Ferner haben viele Unternehmen ihre weit verzweigten Kundendienstabteilungen zu eigenverantwortlichen, leistungsfähigen Geschäftsbereichen zusammengefaßt.

5 Dies trifft besonders auf die hier betrachteten, eher hochwertigen Investitionsgüter zu. Vgl. Schönrock, A., Die Gestaltung des Leistungsmix im marktorientierten Kundendienst, in: Kundendienst-Management, Hrsg.: Meffert, H., Frankfurt/M., Bern 1982, S. 83.

6 Vgl. Benkenstein, M., High Tech-Marketing, in: DBW, 50. Jg., 1990, S. 398.

7 Vgl. Meffert, H., Marktorientierte Führung von Dienstleistungsunternehmen - neue Entwicklungen in Theorie und Praxis, Arbeitspapler Nr. 78 der Wissenschaftlichen Gesellschaft für Marketing und Unternehmensführung e.V., Hrsg.: Meffert, H., Wagner, H., Backhaus, K., Münster 1993, S. 1.

8 Backhaus, K., Investitionsgütermarketing, a.a.O., S. 230. 
Kennzeichnend für den servicepolitischen Bedeutungswandel ist darüber hinaus, daß gerade im Investitionsgüterbereich häufig nicht mehr die eigentlichen Hauptleistungen, sondern bereits Service- und Kundendienstprodukte die dominierenden Umsatz- und Gewinnträger darstellen. ${ }^{9}$

Die aktuelle Bedeutung der Servicepolitik ist jedoch vor dem Hintergrund eines relativ langen Entwicklungsprozesses zu verstehen. Hierbei lassen sich drei Phasen abgrenzen: 10

\section{Kundendienst als "unerwünschte Nebenleistung"}

Nach dem Ende der beiden Weltkriege war das wirtschaftliche Geschehen geprägt durch die Marktmacht der Anbieter (Verkäufermarktsituation). Angesichts einer zunehmenden technischen Komplexität vieler Produkte konnten Reparaturarbeiten in der Regel nicht mehr eigenständig von den Anwendern durchgeführt werden. Die Aufgabe des Kundendienstes bestand daher ausschließlich in der Funktionssicherung von Gebrauchsgütern. Der herrschenden Auffassung nach waren "gute" Produkte jedoch frei von Defekten und ausfallsicher. Demzufolge war das Kundendienstimage in dieser Zeit ausgesprochen negativ. Kundendienstleistungen galten stets als Zugeständnis einer schlechten Produktqualität. Sie wurden deshalb zumeist auch unentgeltlich und freiwillig erbracht.

2. Kundendienst als "Zusatzleistung"

Veränderte Wettbewerbsverhältnisse, zunehmende Produkt- und Markenkonkurrenz sowie nicht zuletzt steigende Ansprüche und differenziertere Kundenwünsche veränderten das Bild des Kundendienstes in den $60 e r$

9 Vgl. Zerr, K., Jugel, S., Dienstleistungen als strategisches Element eines TechnologieMarketing, in: Marketing ZFP, 1989, Nr. 3, S. 164; Quinn, J.B., Baruch, J.J., Paquette, P.C., Exploiting the Manufacturing-Service Interface, in: Sloan Management Review, 1988, No. 4, S. $49 \mathrm{ff}$; Wegwart, J., Preis- und Kontrahierungspolitik im Kundendienst unter besonderer Berücksichtigung von Wartungs- und Call-Service, in: Kundendienst-Management, Hrsg.: Meffert, H., Frankfurt/M., Bern 1982, S. 116 f.; o.V., Marktforscher warnen die Industrie: PCGeschäft wird härter, in: Chip, 1990, Nr. 11, S. 42; Input, Value Added Customer Service Products, 0.O. 1985, S. 1; o.V., Das Geschäft der 90er Jahre, in: Chip, 1991, Nr. 7, S. 11; o.V., Service bringt das Geld, in: Chip, 1991, Nr. 4, S. 13.

10 Vgl. zur Entwicklungsgeschichte des Kundendienstes auch: Meffert, H., Der Kundendienst als Marketinginstrument - Einführung in die Problemkreise des Kundendienst-Managements, in: Kundendienst-Management, Hrsg.: Meffert, H., Frankfurt/M., Bern 1982, S. 2 f.; Frisch, W., Service-Management, Wiesbaden 1989, S. 1 ff.; Weber, M.R., Erfolgreiches Service-Management: Gewinnbringende Vermarktung von Dienstleistungen, Landsberg am Lech 1989, S. 34. 
Jahren (Käufermarktsituation). Besonders seine akquisitorische und absatzpolitische Bedeutung wurde erkannt. Er bekam den Charakter einer Zusatzleistung und diente den Unternehmen als Differenzierungsinstrument gegenüber den Mitbewerbern. In dieser Zeit wurden eine Vielzahl unterschiedlicher Leistungsvarianten entwickelt. Steigende Kundendienstbereitschafts- und -ausführungskosten relativieren jedoch weiterhin noch sein insgesamt verbessertes Image.

3. Kundendienst als "eigenständiger Leistungsträger"

Zu seiner heutigen Bedeutung wandelte sich der Kundendienst Mitte der 70er Jahre. Eine tadellose Produktqualität wird inzwischen als selbstverständlich angesehen. Darüber hinaus werden die Hersteller zunehmend mit immateriellen Leistungsanforderungen der Kunden konfrontiert. Gerade die Anwender komplexer Technologien sind daran interessiert, daß Produkte Nutzen stiften, weniger jedoch, wie dies geschieht. ${ }^{11}$ Levitt stellt hierzu fest, daß Nachfrager zunehmend "Erwartungen" kaufen und keine Erzeugnisse. Der "erwartete Nutzen" wird damit auch zum Maßstab der Kundenzufriedenheit. 12 Die klassischen Prinzipien der Unentgeltlichkeit und des Zusatzcharakters sind inzwischen vollständig zugunsten einer auf Gewinnerzielung ausgelegten Kundendienstpolitik gewichen. Diese orientiert sich heute mehr an den umfassenden und komplexen Bedürfnissen der Anwender, als an der Funktionsfähigkeit und Qualität der Produkte. In der Konsequenz hat sich der Kundendienst damit zu einer zunehmend selbständigen Absatzleistung entwickelt, die mit eigenem Gewinnanspruch allen Risiken und Chancen einer differenzierten Marktleistungspolitik gegenübersteht. 13

11 Vgl. Kotler, P., Marketing Management, 4. Aufl., Englewood Cliffs, N.J., 1980, S. 141 f.; Hampl, R.K., Zunehmende Bedeutung des technischen Kundendienstes (TKD), in: Maschinenbau-Nachrichten (NDMA), 1988, Nr. 9, S. 7; Egli, M., Stutz, H.-R., Es lebe die Kundennutzenforschung, in: io-Management Zeitschrift, 58. Jg., 1989, Nr. 1, S. 48.

12 Vgl. Levitt, Th., Der Verkaufsabschluß ist erst ein Anfang, in: HARVARDmanager, 1985, Nr. 1. S. $16 \mathrm{f}$.

13 Vgl. Meffert, H., Der Kundendienst als Marketinginstrument, a.a.O., S. 3; Weber, R., Erfolgreiches Service-Management, a.a.O., S. $34 \mathrm{f}$. 


\section{Kundendienst als Gegenstand der Marktsegmentierung}

\subsection{Kundendienst als Spezialfall des investiven Dienstleistungsmarketing}

Die wissenschaftliche Auseinandersetzung mit dem Begriff Dienstleistung hat bislang zu keiner einheitlichen Definition geführt. Bezüglich zentraler konstitutiver Merkmale und Besonderheiten von Dienstleistungen gegenüber Sachleistungen besteht jedoch weitestgehend Einigkeit. Dies betrifft die Immaterialität der Leistung, die Leistungsbereitstellung des Dienstleistungsanbieters und die Integration eines externen Faktors (Kunde und/oder Objekt). ${ }^{14}$

Anhand kontextspezifischer Aspekte können Dienstleistungen systematisiert werden (vgl. Abbildung 1). ${ }^{15}$ Zum einen sind dies Leistungen, die im sog. Dienstleistungs- bzw. Servicesektor angeboten werden. Diese Dienstleistungen werden auch als "Primärdienstleistungen" bezeichnet (z.B. Versicherungs- und Bankgeschäfte, Unternehmensberatung). Sie stellen eigenständige, marktfähige Absatzleistungen dar. Im weiteren Verlauf der Arbeit sind Primärdienstleistungen nicht Gegenstand der Untersuchung.

Zum anderen existieren Dienstleistungen, deren eigentlicher Zweck in der Förderung einer übergeordneten Hauptleistung besteht. Diese Dienstleistungen sind häufig integraler Bestandteil eines Systemangebotes, dessen Kernelement aus einer Sachleistung besteht. Ein Hauptleistungsverbund ist damit imma-

14 Zum aktuellen Stand der Dienstleistungsdiskussion vgl. insbesondere Meffert, H., Marktorientierte Führung von Dienstleistungsunternehmen, a.a.O., S. 2 f. Zur Begriffsdiskussion und zu den generellen Besonderheiten von Dienstleistungen u.a. Hilke, W., Dienstleistungs-Marketing, Schriften zur Unternehmensführung, Bd. 35, Hrsg.: Jacob, H., U.a., Wiesbaden 1989, S. 6 ff.; Corsten, H., Betriebswirtschaftslehre der Dienstleistungsunternehmungen, München u.a. 1988, S. 15 ff.; Meyer, A., Dienstleistungsmarketing, 3. Auff., Augsburg 1988, S. 5 ff.; Scheuch, F., Dienstleistungsmarketing, München 1982, S. 2 ff.; Schwenker, B., Dienstleistungsunternehmen im Wettbewerb, Wiesbaden 1989, S. 26 ff.; Meffert, H., Marketing, 7. Aufl., Wiesbaden 1986, S. 43 ff.; Forschner, G., Investitionsgüter-Marketing mit funktionellen Dienstleistungen, Berlin 1989, S. 70 ff.; Büker, B., Qualitätsbeurtellung investiver Dienstlelstungen, Frankfurt/M. U.a. 1991, S. 4 ff.

15 Vgl. Haller, M., Kundenorientierung zwischen High Tech und High Touch, in: THEXIS, 1986, Nr. 1, S. 10. 
nent. 16 Demzufolge lassen sich diese Leistungen auch als "Sekundärdienstleistungen" bezeichnen. 17

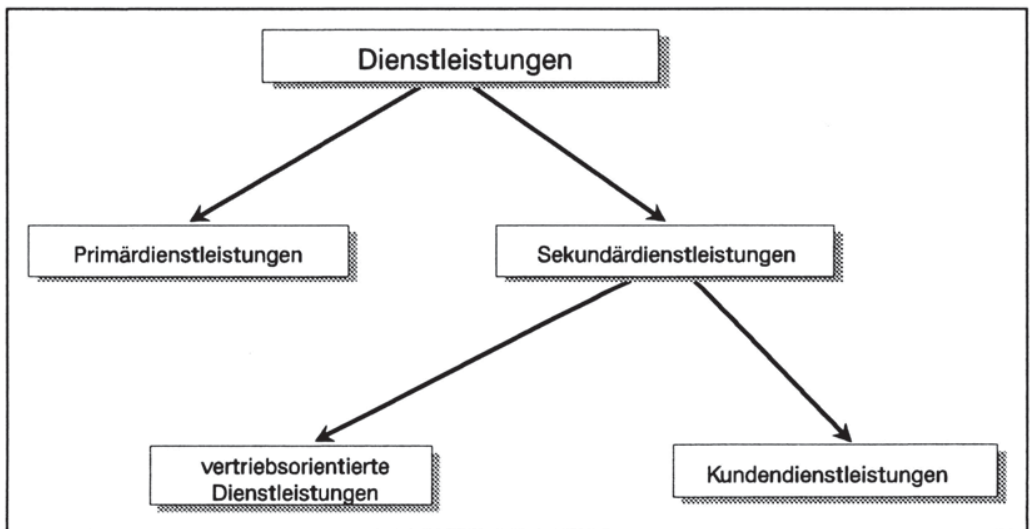

Abbildung 1: Systematisierung von Dienstleistungsarten

Unterschiede zwischen Primär- und Sekundärdienstleistungen ergeben sich insbesondere durch die Aspekte "Immaterialität" und "Selbständigkeit der Absatzleistung". Zum einen bestehen Sekundärdienste im Gegensatz zu Primärdiensten i.d.R. aus einer Kombination von materiellen (Ersatzteile, Dokumentationen, Arbeitsmaterialien) und immateriellen Elementen, wenn auch der immaterielle Anteil vielfach dominiert. 18 Zum anderen werden sie aufgrund des Hauptleistungsverbundes von den Anbietern zumeist nicht als eigenständige Marktleistungen betrachtet und behandelt. Die daraus folgende Unterordnung des Dienstleistungs- unter das Hauptleistungsmarketing führt jedoch häufig zu einem wenig flexiblen und nicht marktorientiert ausgerichteten (Sekundär-) Dienstleistungsmanagement.

16 Vgl. Zerr, K., Jugel, S., Dienstleistung als strategisches Element eines Technologie-Marketing, Arbeitspapier Nr. 68 des Instituts für Marketing der Universität Mannheim, Mannheim 1989 , S. 7.

17 Ebenda, S. $10 \mathrm{f}$.

18 Vgl. Gerstung, F., Die Servicepolitik im Großhandel, Göttingen 1975, S. 16 f.; Belz, Ch., Kundendienst als Marketinginstrument, in: THEXIS, 1986, Nr. 1, S. 3. 
Die Aufgabe von Sekundärdienstleistungen besteht darin, vor, während und nach dem Kauf eines Investitionsgutes ${ }^{19}$ dazu beizutragen,

- dessen Funktionsfähigkeit herzustellen bzw. aufrecht zu erhalten und/oder

- Unternehmen in die Lage zu versetzen, ihr gekauftes Gut optimal zu nutzen und/oder

- das Problemlösungspotential des Produktes durch die Schaffung von Zusatznutzen zu erweitern. 20

Darüber hinaus stellen sie bei technologisch sehr ähnlichen Produkten für Hersteller eine zentrale Chance zur eigenständigen Profilierung gegenüber Wettbewerbern dar. ${ }^{21}$ In diesem Zusammenhang sind vor allem Services von Interesse, die Leistungen über die Herstellung der Funktionalität hinaus beinhalten. Für eine kundenspezifische Servicegestaltung ist dabei jedoch ein vom Hauptleistungsmarketing getrenntes Dienstleistungsmanagement erforderlich.

Sekundärdienstleistungen lassen sich weiter in vertriebsorientierte Dienste und Kundendienstleistungen differenzieren. Dabei umfassen die erstgenannten sämtliche Dienstleistungen, die vor dem eigentlichen Kaufzeitpunkt der Hauptleistung erbracht werden (Pre-Sales-Services). Typische Beispiele sind Beratung, Informationsveranstaltungen u.a. Diese "Vor-Kauf-Dienste" sind ebenfalls nicht Gegenstand der weiteren Ausführungen.

Kundendienstleistungen stehen dagegen im Mittelpunkt der Untersuchung. Sie werden im Rahmen dieser Arbeit wie folgt definiert:

Kundendienstleistungen stehen immer in Bezug zu einem bereits vollzogenen oder beabsichtigten Kauf einer anderen, gleichsam übergeordneten Leistung (Primär- oder Hauptleistung). Diese stellt zunāchst den eigentlichen Mittelpunkt einer Kaufentscheidung dar. ${ }^{22}$

19 Obwohl die geschilderten Zusammenhānge in hohem Maße auch für langlebige Konsumgüter zutreffen, beschränken sich die weiteren Ausführungen aufgrund der Themensetzung aut die Situation von Investitionsgütern.

20 Vgl. Backhaus, K., Investitionsgütermarketing, a.a.O., S. 229.

21 Vgl. Abratt, R., Industrial Buying in High-Tech Markets, in: Industrial Marketing Management, 1986, Nr. 15, S. 297.

22 Vgl. Bennewitz, H.I., Die Eigenständigkeit des absatzpolitischen Instrumentes Kundendienst und seine Bedeutung im modernen Marketingdenken, München 1968, S. 74. 
Versteht man die Hauptleistung als ursprünglich problemlösendes und nutzenstiftendes Gut, so umfassen Kundendienstleistungen alle After-Sales-Servicelejstungen, die sich auf die Sicherstellung, Wiederherstellung und Ausweitung des Gebrauchsnutzens der Hauptleistung beziehen. 23

Das Leistungsspektrum des Kundendienstes umfaßt somit neben den klassischen technischen Kundendienstleistungen (z.B. Installation, Wartung und Reparatur) auch kaufmännische (z.B. Lieferung, Schulungen, Gewährleistungsvereinbarungen) und lösungsbezogene Serviceleistungen (z.B. Anlagenverwaltung, Lösungsdesign und -management). 24 Diese Abgrenzung entspricht in ihren wesentlichen Merkmalen der heute vorherrschenden Auffassung des Kundendienstbegriffs. 25

Die wissenschaftliche Auseinandersetzung mit kundendienstpolitischen Fragen läßt sich analog zur geschichtlichen Entwicklung in drei Phasen einteilen. 26

23 Die Diskussion um den Kundendienst- und Servicebegriff wird in der wissenschaftlichen Literatur kontrovers geführt. Angesichts der Vielzahl unterschiedlicher Interpretationen erscheint es nicht sinnvol eine weitere Variante zu entwickeln. Statt dessen soll mit der hier gewählten Definition eine Grundlage gelegt werden, die sich durch ihre Breite und Anpassungsfähigkelt und damit ihre praktischen Anwendungsmöglichkeiten auszeichnet. Im folgenden werden deshalb die Begriffe "Kundendienst(-leistung)" und "Service(-leistung)" synonym verwendet. Damit wird der zunehmend festzustellenden Gleichsetzung dieser Begriffe in der Praxis entsprochen. Zu einer ausführlichen begrifflichen Literaturdiskussion vgl. besonders Frisch, W., Service-Management, a.a.O., S. $112 \mathrm{ff}$. und die dort angegebene Literatur.

24 Töpfer spricht im Zusammenhang mit der Typisierung von Kundendienstleistungen von einer Entwicklung der "Hardware"-, über die "Software"- hin zu "Solutionware"-Diensten. Vgl. Töpfer, A., Marketing für Start-up-Geschäfte mit Technologieprodukten, in: Technologie Marketing, Hrsg.: Töpfer, A., Sommerlatte, T., Landsberg am Lech 1991, S. 195.

25 Wesentliche Vertreter der zugrundegelegten Kundendienst-Definition sind: Frisch, W., Service-Management, a.a.O., S.112 ff.; Weber, M.R., Erfolgreiches Service-Management, a.a.O., S.27 ff.; Meffert, H., Der Kundendienst als Marketinginstrument, a.a.O., S. 2. ff.; Meffert, H., Kundendienstpolitik, a.a.O., S. 93; Zerr, K., Jugel, S., Dienstleistung als strategisches Element eines Technologie Marketing, a.a.O., S. 6 f.; Haller, M., Kundenorientierung zwischen High Tech und High Touch, a.a.O., S. 10.; Gerstung, F., Die Servicepolitik als Instrument des Handelsmarketing, Göttingen 1978; Konrad, E., Kundendienstpolitik als Marketinginstrument von Konsumgüterherstellern, Zürich 1974, S. 9; Bazzi, R., Pelz, N., Investitionsgüterservice Direkt am Puls des Marketing, in: THEXIS, 1986, Nr. 1, S. 15.

26 Streng genommen läßt sich der Ursprung kundendienstpolitischer Auseinandersetzungen bis zum Beginn dieses Jahrhunderts zurückverfolgen. Zu dieser Zeit wurde mit Kundendienst noch vornehmlich ein Geschäftsprinzip assoziiert - der Dienst am Kunden. Vgl. hierzu z.B. Jacoby, A., Kundendienst - Der erfolgreiche Verkäufer im Detailhandel, Schweizer Schriften für rationelles Wirtschaften, Nr. 4, Zürich 1927 S. 8 f. 
Intensive begriffliche Grundlagenarbeit kennzeichnete die Diskussion in den 60er und 70er Jahren. 27 Im Mittelpunkt stand dabei der Aspekt einer Kundendienstleistung als "Neben- bzw. Zusatzleistung", die erforderlich ist, um den Absatz der Hauptleistung zu ermöglichen bzw. zu fördern. ${ }^{28}$ Servicepolitik wurde dabei zumeist gleichgesetzt mit technischem Kundendienst.

Wesentliche Veränderungen in der wissenschaftlichen Auseinandersetzung erfolgten zu Beginn der 80er Jahre. Zunehmend wurden Aspekte eines umfassenden Kundenmanagements entwickelt und diskutiert. 29 Diese Phase ist gekennzeichnet durch ein Verständnis des Kundendienstmanagements als zielbezogener und marktgerichteter Entscheidungsprozeß, der sich mit der "gegenwärtigen und zukünftigen Gestaltung von Serviceleistungen und deren Zusammensetzung zu einem attraktiven Serviceprogramm" beschäftigt. 30

Neben der Etablierung einer entscheidungs- und marktorientierten Denkweise in der Kundendienstpolitik, stammen aus dieser Zeit eine Reihe von Arbeiten, die sich mit Detailproblemen auseinandersetzten. Schwerpunkte stellten dabei insbesondere kundendienstbezogene Prognose-, Organisations- und Steuerungssowie Optimierungs-, Interdependenz- und Koordinationsprobleme dar. 31

27 Vgl. Bennewitz, H.J., Die Eigenständigkeit des absatzpolitischen Instrumentes Kundendienst und seine Bedeutung im modernen Marketingdenken, a.a.O., S. 85 f.; Theisen, P., Kundendienstpolitik, in: HWA, Hrsg.: Tietz, B., Stuttgart 1974, Sp. 1155 ff.; Konrad, E., Kundendienstpolitik als Marketing-Instrument von Konsumgüterherstellern, a.a.O., S. 9 f.; Koch, W., Grundlagen und Technik des Vertriebes, Bd. 1, Berlin 1958, S. 720; Gerstung, F., Die Servicepolitik im Großhandel, a.a.O., S. 21 f.

28 Vgl. Frisch, W., Service-Management, a.a.O., S. $115 \mathrm{f}$.

29 Vgl. hierzu besonders: Weber, M.R., Erfolgreiches Service-Management, a.a.O.; Frisch, W., Service-Management, a.a.O., Forschner, G., Investitionsgüter-Marketing mit funktionellen Dienstleistungen, a.a.O. u.a.

30 Meffert, H. , Kundendienstpolitik, a.a.O., S. 93.

$31 \mathrm{Zu}$ Prognosemöglichkeiten in der Kundendienstpolitik vgl. Lo, L., Prognoseinformationen für kundendienstpolitische Entscheidungen, Münster 1979; zu Organisations- und Steuerungsaspekten vgl. Fußbahn, K.-H., Organisation und Steuerung des Technischen Kundendienstes - Profit- oder Costcenter?, in: Kundendienst-Management, Hrsg.: Meffert, H., Frankfurt/M., Bern 1982, S. 125 ff.; zu Optimierungsproblemen vgl.: Hammann, P., Das Optimierungsproblem im Kundendienst - Aussagewert und Stand der Diskussion, in: KundendienstManagement, Hrsg.: Meffert, H., Frankfurt/M., Bern 1982, S. 145 ff.; zu Interdependenz- und Koordinationsaspekten vgl. Meffert, $\mathrm{H}$., Interdependenzen und Koordination zwischen Marketing und Kundendienst, in: Kundendienst-Management, Hrsg.: Meffert, $H$., Frankfurt/M, Bern 1982, S. $171 \mathrm{ff}$. 
In der modernen Literaturauffassung wird der Kundendienst zunehmend als eine der zentralen Schlüsselgrößen im gesamten Unternehmensprozeß diskutiert. 32 Im Mittelpunkt stehen hierbei insbesondere die Möglichkeiten des Service in bezug auf Kundenzufriedenheit und Kundenbindung. Diese Konzepte werden vielfach unter dem Stichwort einer "Zero-Migration" diskutiert. 33 Auch Gesichtspunkte der Kundendienstqualität ${ }^{34}$ sowie die kundendienstpolitischen Möglichkeiten im Rahmen eines Total Quality Managements finden zunehmend Beachtung. 35

\subsection{Kundendienstlelstungen als elgenständige Absatzleistung}

Vor einer differenzierteren Diskussion möglicher Segmentierungsansätze ist es notwendig, das Serviceangebot zu strukturleren, um einen Überblick über das Spektrum zu analysierender Kundendienstprodukte zu erhalten. In Theorie und Praxis gleichermaßen anerkannt ist dabei eine Klassifizierung von Kundendienstleistungen in "MUß"-, "Soll"- und "Kann"-Dienste. Obgleich aufgrund rechtlicher, soziokultureller, wirtschaftlicher und technischer Rahmenbedingungen die Freiheitsgrade des servicebezogenen Leistungsmix recht verschieden sind, ${ }^{36}$ las-

32 Vgl. Graumann, J., Gebhardt, P., Die Managementaufgabe der Zukunft: Dienstleistungsmarketing, in: Markenartlkel, 1987, Nr. 10, S. 486 ff.; Quinn, J.B., Gagnon, Ch.E., Dle Dienstlelstungen werden automatisiert, In: HARVARDmanager, 1987, Nr. 2; S. 74 ff.; Ghemawat, P., Dauerhafte Wettbewerbsvortelle aufbauen, In: HARVARDmanager, 1987, Nr. 2., S. 104 ff.

33 Vgl. Reichheld, F.F., Sasser, W.E., Zero-Migration: Dlenstleister im Sog der Qualitätsrevolutlon, In: HARVARDmanager, 1991, Nr. 4, S. 108 ff.; Hart, Ch.W., Heskett, J.L., Dasser, W.E., Wie Sie aus Pannen Proftt zlehen, In: HARVARDmanager, 1991, Nr. 1, S. 128 ff.; Lingenfelder, M., Schnelder, W., Die Kundenzufriedenhelt, in: Marketing ZFP, 1991, Nr. 2, S. 109 ff.; Lovelock, Ch., Dienstleister können Efflzienz und Kundenzufrledenhelt verbinden, In: HARVARDmanager, 1993, Nr. 2, S. $68 \mathrm{ff}$.

34 Vgl. Stauss, B., Kundendienstqualităt als Erfolgsfaktor im Wettbwerb, In: THEXIS, 1991, Nr. 2, S. 47 ff.; Buker, B., Qualtätsbeurtellung Investlver Dienstleistungen, a.a.O., S. 1 ff.

35 Schlesinger, L.A., Heskett, J.L., The Service-Drlven Service Company, in: Harvard Business Revlew, 1991, No. 10/11, S. 71 ff.; Braun, K., Lawrence Ch., TQM I: Ohne gemeinsame Werte wird es nichts, In: HARVARDmanager, 1993, Nr. 2, S. 77 ff.; Raffio, T., TQM II: Wie Delta Dental Plan Service Excellence erreichte, in: HARVARDmanager, 1993, Nr. 2, S. 86 ff.

36 So hat z.B. Jeder Käufer hinsichtlich der Funktionalltăt elnes Neuproduktes einen gesetzllch definlerten Gewährleistungsanspruch (z.B. eine halbjährlge Gewăhrleistungszelt für elnen Automotor). In der Konsequenz sind Hersteller damit zur Bereltstellung grundlegender Kundendienstleistungen verpflichtet. Darüber hinaus obliegt es jedoch jedem Anbleter selbst, diese Leistungsverpflichtung durch frelwillige Garantlezusagen zu erweltern (z.B. elne 
sen sich durch einen zielgruppengerechten, d.h. segmentgerechten Zuschnitt von Kundendienstleistungen zusätzliche Erfolgspotentiale ausschöpfen.

Zu den "Muß"-Leistungen gehören Leistungen, die eine Nutzung des gekauften Produktes überhaupt ermöglichen, d.h. die Hauptleistungsfunktion erstmalig herstellen. Ferner sind dieser Gruppe Services zuzuordnen, die aus verkaufstechnischen bzw. marktbedingten Gründen vom Anbieter erbracht werden müssen (z.B. Lieferung, Aufbau, Installation, Gewährleistungsverpflichtungen und Garantiezusagen).

Zu der Gruppe der "Soll"-Dienste werden Leistungen gerechnet, die aufgrund von Zuverlässigkeitsschätzungen und Prognosen (Materialermüdung, Lebensdauer bestimmter Bauteile) vorhersehbar sind. 37 Dazu zählen vorrangig alle Wartungs- und Reparaturleistungen. Gerade in diesem Bereich sind viele Hersteller seit geraumer Zeit bemüht, ihren Käufern Soll-Leistungen in attraktiveren Serviceformen anzubieten. So werden in der Computerindustrie z.B. seit Jahren Kundendienstverträge angeboten, die neben den originären Soll-Leistungen je nach Form eine Fülle von Zusatzleistungen umfassen (z.B. garantierte Reaktionszeiten, 24-Stunden-Services, Versicherungsschutz u.a.). 38

Während das Leistungsspektrum der Muß- und Soll-Leistungen vorwiegend zu den "klassischen" Kundendienstleistungen zählt, ordnet man die "Kann"-Leistungen der Gruppe der additiven Dienstleistungen zu. Sie sind im hohen Maße nachfragebedingt und hängen von den spezifischen Bedürfnissen der Hauptleistungs-Anwender ab. ${ }^{39}$ Stellen die klassischen Services in erster Linie die zugesagte Funktion der Hauptleistung her bzw. sichern diese ab, zielt das Angebot von "Kann"-Leistungen auf die Schaffung von Zusatznutzen beim Anwender. In Abhängigkeit von den individuellen Kosten-/Nutzen-Vorstellungen der Hauptleistungsnehmer ergeben sich hierbei Chancen für die Entwicklung zielgruppenspezifischer Kundendienstprodukte. Abbildung 2 faßt die Typologie von Kundendienstleistungen am Beispiel von Computersystemen zusammen.

sechsjährige Garantie gegen Rostschăden an einer Autokarosserie). Vgl. hlerzu $\$ \S 433$ ff., BGB.

37 Vgl. Meffert, H. , Kundendienstpolltik, a.a.O., S. 97; Lo, L., Prognoseinformationen für kundendienstpolitische Entscheidungen, a.a.O., S. 28.

38 Vgl. Wegwart, J., Preis- und Kontrahierungspolitik im Kundendienst unter besonderer Berücksichtlgung von Wartungs- und Call-Service, a.a.O., S. 121 f.

39

Vgl. Meffert, H., Kundendienstpolitik, a.a.O., S. 97. 


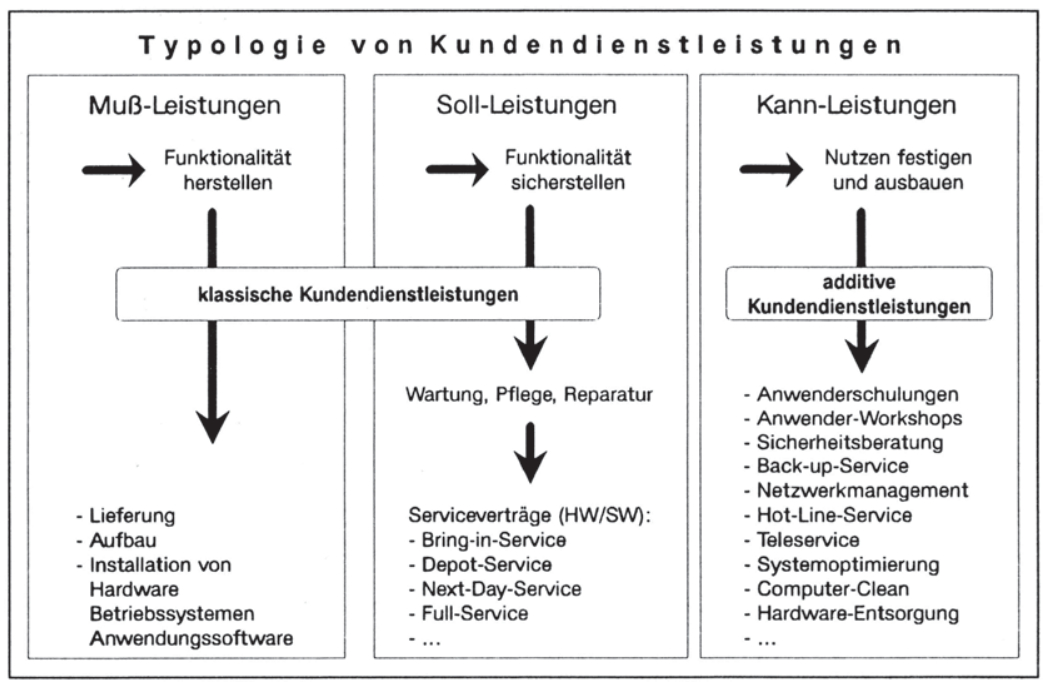

Abbildung 2: Typologie von Kundendienstleistungen am Beispiel von Computerservices

Die vorgestellte Typisierung von Kundendienstleistungen bietet eine klare Einteilung und Systematisierung verschiedener Einzelservices. Als Trennungskriterium zwischen eigenständig marktfähigen und dem Hauptleistungsmarketing beigeordneten Serviceleistungen ist diese Kategorisierung jedoch nicht geeignet.

Besonders aufgrund des in Investitionsgütermärkten eher rationalen Kaufverhaltens werden Unternehmen, die neue Technologien erwerben, neben deren Anschaffungskosten auch die antizipierbaren Pflege- und Wartungskosten in ihre Investitionüberlegungen einbeziehen. Diese "life-cycle-costs"-orientierte Betrachtungsweise führt z.B. beim Kauf von Computersystemen häufig dazu, daß das eigentliche Kaufpaket nicht nur aus Hard- und Softwarekomponenten besteht, sondern den unternehmensbezogenen Erwartungen und Erfahrungsstrukturen entsprechende Dienstleistungen bereits mit eingeschlossen werden. 40 Derartige Leistungen bestehen i.d.R. aus Transport- und Installationsdiensten. Jedoch auch Wartungs- und Serviceverträge werden vielfach beim Kaufabschluß der

40 Zum Konzept des Life-Cycle-Costing vgl. Backhaus, K., Investitionsgütermarketing, a.a.O., S. 455 ff.; Finkelstein, W., Guertin, R.J.A., Integrated Logistics Support - The Design Engineering Link, Berlin u.a. 1988, S. $167 \mathrm{ff}$. 
Hauptleistung vereinbart und gehören damit faktisch zum Lösungspaket. Die wettbewerbs- und zielgruppengerechte Zusammenstellung attraktiver Lösungspakete, und damit gleichzeitig verbunden auch die Auswahl und der Umfang entsprechender Serviceleistungen, ist Aufgabe des Produkt-, d.h. Hauptleistungsmarketing. Fragen der Markteinteilung, -wahl und -bearbeitung sowie die jeweils geeignete Angebotszusammenstellung (Hardware, Software und Service) fallen damit in dessen Aufgabenbereich.

Services, die aufgrund fehlender Erfahrungen nur teilweise bzw. gar nicht antizipiert wurden, oder die nicht antizipierbar sind (z.B. die notwendige Schulung neuer Mitarbeiter, die durch den Ausfall erfahrener Mitarbeiter erforderlich wird), müssen durch das Unternehmen zu einem späteren Zeitpunkt erworben werden. Sie unterliegen damit eigenständigen Marktprozessen.

Problematisch ist bei dieser Abgrenzung, daß bestimmte Services nicht eindeutig zugeordnet werden können, da die Zuordnung vom situativen Kontext der Hauptleistungskaufentscheidung abhängt. Damit offenbart sich die besondere Schwierigkeit der Kaufprozeßzuordnung bzw. -abgrenzung von Serviceleistungen. Einerseits sind Dienstleistungen regelmäßig der Produktleistung im Sinne von "value added service" beigefügt. ${ }^{41}$ Derartige Kundendienste sollen den Nutzen und die Attraktivität des "Hauptleistungspakets" erhöhen und damit Präferenzen gegenüber anderen Angeboten beim Kunden aufbauen. Solche Leistungen können als "Grundservices" bezeichnet werden. In bezug auf den Entscheidungsprozeß der Nachfrager sind sie zeitlich und inhaltlich eindeutig dem Primärleistungskauf zuzurechen. Segmentierungsbezogene Fragestellungen unterliegen damit dem Hauptleistungsmarketing und werden deshalb auch aus der weiteren Untersuchung ausgegrenzt. 42

41 Zum "value added"-Begriff vgl. Remmerbach, K.U., Value Added Marketing, in: Marktforschung \& Management, 1990, Nr. 2, S. 13 ff.; Mertens, P., Schumann, M., Hohe, U., Informationstechnik als Mittel zur Verbesserung der Wettbewerbsposition - Erkenntnisse aus einer Beispielsammlung, in: Informationstechnologie und strategische Führung. Hrsg.: Spremann, K., Zur, E., Wiesbaden 1989, S. 124 ff.; Cordroch, C., Kundendienst unter Druck, in: Online, 1988, Nr. 1, S. 25; Berry, D., Value added marketing emerging as key to competitiveness, in: Marketing news, 1987, Nr. 7, S. 3 ff.; Meyer, A., Produktdifferenzierung durch Dienstleistungen, in: Marketing ZFP, 1985, S. 99 ff.; Backhaus, K., Weiber, R., Systemtechnologie - Herausforderung des Investitionsgütermarketing, in: HARVARDmanager, 1987, Nr. 4, S. $70 \mathrm{ff}$.

42 Die Ausgrenzung der Grundservices für die weitere Untersuchung im Bereich der Marktsegmentierung für Kundendienstleistungen korrespondiert nicht mit der faktischen organisationsbezogenen Verantwortung im Unternehmen. So wird ein eigenständiger Kundendlenst- 


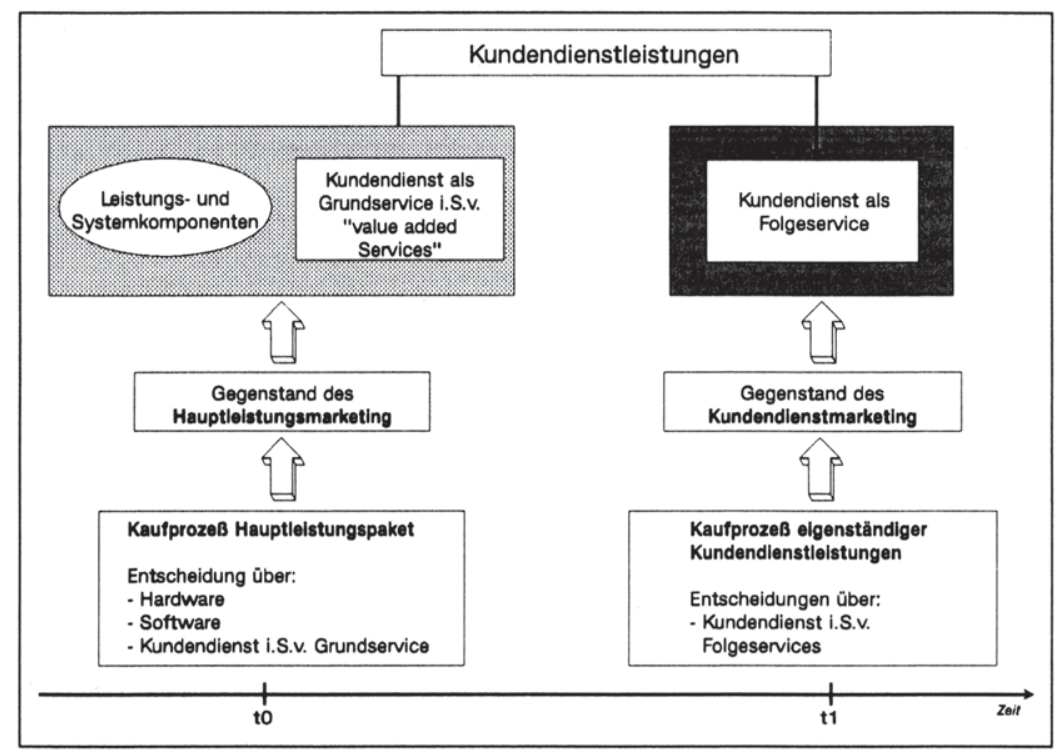

Abbildung 3: Zuordnung von Serviceleistungen zu verschiedenen Kaufentscheidungssituationen

Andererseits besteht eine Gruppe eigenständiger Kundendienstleistungen, deren Nachfrage überhaupt erst aufgrund der Nutzungs- und Einsatzerfahrung mit der Hauptleistung entsteht. Diese Leistungen stehen im Mittelpunkt der weiteren Ausführungen. Der Bedarf an solchen "Folgeservices" entwickelt sich erst im Laufe der Hauptleistungsnutzung und unterliegt damit vollständig eigenen Kaufprozessen (vgl. Abbildung 3). 43

bereich für alle Services verantwortlich sein. Daraus resultiert einerselts eine klare Organisationsstruktur. Andererselts ergeben sich aber, insbesondere wenn der Bereich als ProfitCenter geführt wird, in der Praxis Probleme der internen Leistungsverrechnung, die hier allerdings nicht welter diskutlert werden. Vgl. zur Verrechnungspreisproblematik, Zimmermann, G., Grundzüge der Kostenrechnung, 2. Aufl., Stuttgant 1982, S. 85 ff.; Hummel, S., Mănnel, W., Kostenrechnung 1 - Grundlagen, Aufbau und Anwendung, 4. Aufl., Wlesbaden 1986, S. 30 f.; Schlerenbeck, H., Grundzüge der Betriebswirtschaftlehre, 9. Aufl., München 1987, S. $596 \mathrm{f}$.

43 Z.B. stehen Im Rahmen des Kaứs einer Computeranlage für eln Unternehmen zunächst Fragen der geelgneten Hardware- und Softwarekomponenten Im Miltelpunkt des Entscheidungsprozesses. Daneben werden in bestimmtem Umfang jedoch berelts Kundendienstleistungen in die Kaufüberlegungen einbezogen (Grundservices). So muß die Anlage richtlg aufgebaut und verkabelt werden (Lleferung, Aufbau). Die Betrlebssystem- und Anwendungs- 


\subsection{Marktsegmentlerung von Kundendienstleistungen}

Das Marketing beschäftigt sich bereits seit vielen Jahren mit Fragen der Marktsegmentlerung. Im Mittelpunkt steht dabei die Aufteilung von Gesamtmärkten in homogene Käufergruppen und -segmente. 44 Dabei ist die Segmentierung von Märkten eine zentrale Voraussetzung jeder differenzierten Zielgruppenpolitik. 45 Sowohl Vertreter eines reinen Marktaufteilungsansatzes als auch der strategischen Marktsegmentierung 46 haben sich zunächst vor allem der Erforschung von Konsumgütermärkten zugewandt. 47 Eine Unterteilung des Gesamtmarktes findet mit Hilfe verschiedener Segmentierungsmerkmale statt. Dabei steht das Bestreben im Vordergrund, Kriterien so zu wählen und zusammenzustellen, daß sie den Markt und seine Teilnehmer vor allem in bezug auf das Kaufverhalten eindeutig charakterisieren. Auf dieser Grundlage können dann unterschiedliche Strukturen eines Gesamtmarktes identifiziert und voneinander abgegrenzt werden. 48 Nur wenn Segmentierungskriterien eine dementsprechend hohe Kaufverhaltensrelevanz aufweisen, ist gewährleistet, daß die einzelnen

software ist fachgerecht zu installieren (Installation und Inbetriebnahme). Zudem soll eine erste Elnweisung der Anwender den Umgang mit dem neuen System erlelchtern (Einweisung, Instruktion). Nach einer gewissen Einsatz- und Nutzungszelt der neuen Anlage im Unternehmen, äußern sich zunehmend viele Anwender beim EDV-Beauttragten über Handhabungsprobleme bel einem bestimmten Softwareprogramm. Das Unternehmen wendet sich darauthin an den Kundendienst und vereinbart für seine Anwender ein Schulungsseminar (Folgeservice).

44 Vgl. Meffert, H., Marketing, a.a.O., S. 243.

45 Ebenda, S. $27 \mathrm{f}$.

46 Zu einer Unterscheidung beider Interpretationen vgl. Kap. B.11.

47 Freter, H., Marktsegmentlerung, Stuttgart, Berlin, Köln, Mainz 1983; Bauer, E., Marktsegmentlerung als Marketing-Strategle, Berlin 1976; Kaiser, A., Die Identfflkatlon von Marktsegmenten, Berlin 1978; Böhler, H., Methoden und Modelle der Marktsegmentierung, Stuttgart 1977; Crone, B., Marktsegmentlerung, Bern 1977; Frank, R.E., Massy, W.F., Wind, Y., Market Segmentation, Englewood Cliffs, N.J. 1972; Winter, F.W., A Cost-Benefit Approach to Market Segmentation, In: Journal of Marketing, Vol. 43, 1979, No. 4, S. 103 - 111.; Wind, Y., Issues and Advances in Segmentation Research, In: Journal of Marketing Research, Vo. 15, 1978, No. 8, S. 317 - 337; Martin, C.R., Wright, R.L., Profit-Oriented Data Analysis for Market Segmentation, In: Journal of Marketing Research, Vol. 11, 1974, No. 8, S. 237 - 242; Dichtl, E., Müller-Heimann, G., Konsumententypologische und produktorientlerte Marktsegmentlerung, In: Jahrbuch der Absatz- und Verbrauchsforschung, 1972, Nr. 4, S. 249 - 265.

48 Vgl. Kols, P., Bedarfsorlentlerte Marktsegmentlerung auf Produktlvgütermärkten, Frankfurt/M., 1986, S. 29. 
Marktsegmente im Rahmen von Marktbearbeitungsmaßnahmen auch homogen auf die spezifischen Marketingimpulse eines Anbieters reagieren. 49

In der jüngeren Vergangenheit rücken zunehmend auch Investitionsgütermärkte in den Mittelpunkt segmentierungsbezogener Untersuchungen.50 Aufgrund der kollektiven Entscheidungs- und Kaufstrukturen von Unternehmen erweist sich dabei besonders die Marktaufteilung als Problem. Diesbezüglich sind deshalb Unterschiede in der Buying-Center-Besetzung, im Rollenverhalten sowie den jeweilig bestehenden Einfluß- und Machtstrukturen der am Kaufentscheidungsprozeß beteiligten Unternehmensmitglieder von zentralem Interesse. Erst mit der Einbeziehung dieser zusätzlichen Aspekte wird den Erfordernissen einer Segmentierung auf investiven Märkten entsprochen.

Dienstleistungen, insbesondere Kundendienstleistungen widmeten die Segmentierungsforscher bislang wenig Beachtung. ${ }^{51}$ Eine globale Behandlung von Kundendienstmärkten kann aber kaum zu einer optimalen, kundenorientierten Servicepolitik führen. Vielmehr ist die Identifizierung spezifischer Bedürfnissegmente eine Voraussetzung für eine effiziente, eigenständige Absatzpolitik im Bereich von Kundendienstleistungen. Ein servicebezogener Segmentierungsansatz kann dabei nur vor dem Hintergrund einer Beachtung seiner kennzeichnenden Besonderheiten entwickelt werden. Bestehende Segmentierungskonzepte aus

49 Vgl. Horst, B., Ein mehrdimensionaler Ansatz zur Segmentierung von Investitionsgütermärkten, Köln 1988, S. 131.

50 Kols, P., Bedarfsorientierte Marktsegmentierung auf Produktivgütermärkten, a.a.O.; Gröne, A., Marktsegmentierung bei Investitionsgütern, Wiesbaden 1977; Horst, B., Ein mehrdimensionaler Ansatz zur Segmentierung von Investitionsgütermärkten, a.a.O., Bonoma, T.V., Shapiro, B.P., Segmenting the Industrial Market, Lexington, Mass./Toronto 1983.

51 So spricht Meffert in diesem Zusammenhang auch von einem "Segmentierungsproblem" in der Kundendienstpolitik. Neben der Feststellung, daß natürlich zunächst zu klären sel, ob Kunden gleich oder verschleden zu behandeln sind, weist er besonders auf dle Eignung von Kriterienkomblnationen zur Kundendienstsegmentierung hin. Vgl. hierzu Meffert, H., Der Kundendienst als Marketinginstrument - Einführung in die Problemkreise des KundendienstManagements, a.a.O., S. 23; Meffert, H., Kundendienstpolitik, a.a.O., S. 96. Zu ersten Ansätzen der Dienstleistungs-Segmentierung vgl. Mühlbacher, H., Botschen, G., Benefit-Segmentierung von Dienstleistungsmärkten, in: Marketing ZFP, 12. Jg., 1990, Nr. 3, S. 159 - 168; Davidow, W.H., Uttal, B., So wird ihr Kundendienst unschlagbar, in: HARVARDmanager, 1990, Nr. 2, S. 14 - 21; Lynn, S.A., Segmenting a Business Market for a Professional Service, in: Industrial Marketing Management, Vol. 15, 1986, S. 13 - 21; Thiesing, E.-O., Strategische Marketingplanung in filialisierten Universalbanken. Integrierte Filial- und Kundengruppenstrategien auf der Grundlage erfolgsbeeinflussender Schlüsselfaktoren, Frankfurt/M., Bern, New York, Paris 1986.; Davidow, W.H., High Tech Marketing, Frankfurt/M. 1987. 
anderen Bereichen des Marketing berücksichtigen diese Eigenarten jedoch nur unzureichend.

Auch praxisbezogene Handlungsweisen zur Marktauf- bzw. -einteilung von Kundendienstmärkten scheinen den Anforderungen eines zielgruppengerechten Servicemarketing bislang wenig gerecht zu werden. Die meisten Hersteller ordnen ihre Kundendienstpolitik in marktbearbeitungsstrategischer Hinsicht nach wie vor, zumeist unreflektiert, den Zielgruppenkonzepten ihrer Hauptleistungen unter. Segmentierungsmerkmale und Marktsegmente werden dabei vom Hauptleistungsmarketing unverändert auf die Kundendienstpolitik übertragen. So werden z.B. Märkte für Computersysteme vielfach nach branchenbezogenen Kriterien differenziert, anstatt einer Segmentierung die jeweils spezifische Bedürfnissituation der Unternehmen zugrunde zu legen.

Dabei kann die Branchenzugehörigkeit (Industrie, Handel, selbständige Wirtschaft u.a.) durchaus einen starken Einfluß auf das Anforderungsprofil eines Computersystems als eigentlicher Problemlösung haben. Probleme und damit zwangsläufig verbundene Nutzeneinbußen beim Betreiber resultieren jedoch häufig aus mangelndem Bedienungsverständnis und Anwendungsfehlverhalten. Diesbezüglich bestehen aber bei vielen Unternehmen branchenintern unterschiedliche und branchenübergreifend vergleichbare Voraussetzungen ( $\mathrm{gl}$. Abbildung 4).

Insgesamt bestehen also weder in der Wissenschaft noch in der Praxis geeignete Lösungsansätze für die spezifischen Erfordernisse einer Segmentierung von Kundendienstleistungen i.S.v. Folgeservices.

Zur Segmentierung des Marktes für Folgeservices ist es insbesondere wichtig aufzudecken, welche Bestimmungsfaktoren ein unterschiedliches Kaufverhalten von Serviceleistungen maßgeblich prägen. Hierzu ist vor allem die bedürfnisbezogene Situation der Nachfrager heranzuziehen. 52 Eine Aufgabe der Kundendienstsegmentierung ist damit die Suche nach Indikatoren, die in möglichst engem Bezug zu den tatsächlichen Serviceanforderungen stehen und so die Voraussetzungen für eine sinnvolle Marktaufteilung schaffen. Bei einer investitions-

52 Zum Prozeß der Bedürnisbefriedigung vgl. Hammann, P., Marktforschung für Investitionsgüter, in: AnlagenMarketing, Sonderheft Nr. 7 der ZfbF, Hrsg.: Engelhardt, W.H., Lassmann, G., Opladen 1977, S. 96. 


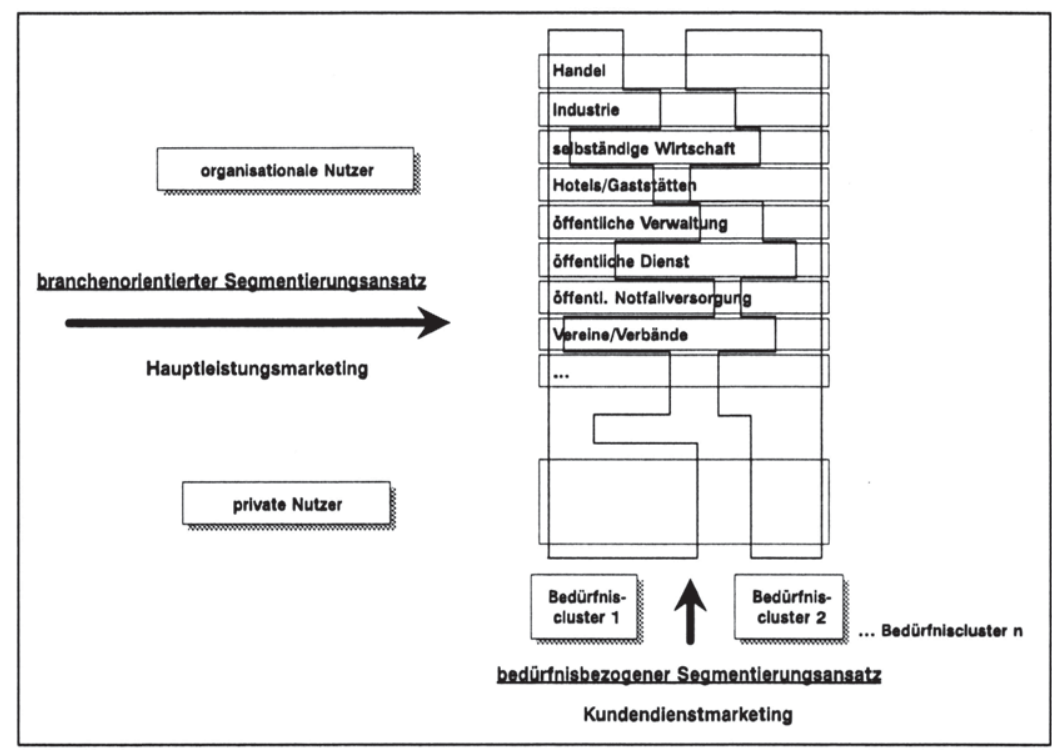

Abbildung 4: Segmentierungsproblematik am Beispiel des Computermarktes

güterbezogenen Betrachtung gehört hierzu auch die Identifikation von zentral bedürfnisprägenden bzw. -bestimmenden Personen in Unternehmen.

Auch hinsichtlich der Struktur und des Ablaufs betrieblicher Kaufprozesse sind Verflechtungen zwischen Kundendienst und Hauptleistung zu berücksichtigen. Im Zusammenhang mit diesen Prozessen gilt es zu klären, inwieweit sich derartige Buying Center-Strukturen beim Hauptleistungskauf und beim Servicekauf unterscheiden bzw. gleichen. 53 Angefangen mit der Frage, welche Mitarbeiterkreise in derartige Entscheidungen überhaupt einbezogen sind, ist zu analysieren, welchen Einfluß bzw. welche Macht diese dann im Rahmen der Entscheidungsfindung haben. So ist auch zu untersuchen, inwieweit diese Kaufentschei-

53 Backhaus definlert das Buying Center eines Unternehmens als die "gedankliche Zusammenfassung aller am Kaufprozeß betelligten Personen". Demnach gehört es zu den zentralen Aufgaben eines Investitlongüteranbleters zunächst herauszufinden, wer die betelligten Personen sind und wie sich dlese im Rahmen dieses Gremlums verhalten (Informations-, Elnfluß-, Entscheidungs- und Anregungsverhalten). Backhaus, K., Investitlonsgütermarketing, 2. Auff., München 1990, S. 32 ff. Vgl. zum Buying Center Modell auch: Meffert, H., Marketlngforschung und Kăuferverhalten, 2. Aufl., Wiesbaden 1992, S. 144 f.; Kratz, J., Der Interakt1onsprozess beim Kauf von einzeln gefertigten Investitionsgütern, Bochum 1975, S. 124 ff. 
dungsstrukturen mit den Anforderungsstrukturen eines Unternehmens im Einklang stehen, d.h. ob bestehende Bedürfnisse auch tatsächlich in konkrete Nachfrage nach Serviceleistungen umgesetzt werden. 54 Dies unterstreicht, daß für eine strategische Kundendienstpolitik außer der Bedarfs- auch die Buying Center-Situation in Unternehmen zu berücksichtigen ist. 55

\section{Der Markt für kommerzlelle Personal Computer als Gegenstand der Untersuchung}

Die Segmentierung von Kundendienstleistungen soll in der vorliegenden Untersuchung am Beispiel des Marktes für kommerzielle Personal Computer (PCs) dargestellt werden. 56 Um die Notwendigkeit einer differenzierten Servicepolitik kennzeichnen zu können, ist es erforderlich, wichtige Entwicklungen im Computermarkt aufzuzeigen, um so zentrale Aufgaben des Kundendienstes ableiten zu können.

Die Bedeutung der Kundendienstpolitik in der Computerbranche ist traditionell sehr hoch. Der zentrale Stellenwert von EDV-Systemen in nahezu allen Aufgabenbereichen von Unternehmen einerseits sowie die hohe technologische Komplexität solcher Lösungen andererseits, hat Computeranbieter frühzeitig dazu veranlaßt, ihren Kunden neben dem Angebot an Hardware- und Softwarekomponenten auch ein umfangreiches Spektrum an Kundendienstleistungen zur Verfügung zu stellen. Eines der Hauptinstrumente bei nahezu allen großen Anbietern und gleichzeitig einer der aussagekräftigsten Indikatoren für die Konjunktur von Serviceleistungen stellt der Kundendienstvertrag dar.

54 Zum "Elnfluß-"und "Macht"-Begrlff Im unternehmensbezogenen Kautverhalten vgl. Backhaus, K., Investitionsgütermarketing, a.a.O., S. 46 ff.; Kern, E., Der Interaktionsansatz Im Investitionsgütermarketing, Berlin 1990, S $41 \mathrm{ff}$.

55 Vgl. hierzu Im auch Kap. B.3.2

56 Personal Computer stellen damit im folgenden die Hauptlelstung dar, auf dle sich die Kun. dendienstleistungen beziehen. Im einzelnen handelt es sich hierbel um PC Systeme, die aus handelsüblichen Hardwarekomponenten (BHdschirm, Tastatur, Zentralelnhelt, Drucker) bestehen und mit PC-ubllcher Betriebssystem-Software (Dos, Windows, OS/2, Unbx, Netzwerksoftware) sowle Standardanwendungs-Software (Textverarbeltung, Tabellenkalkulation, Graflk u.a.) betrleben werden. Welteres Abgrenzungskriterlum ist der Elnsatz dieser Systeme Im betrieblichen, kommerziellen Umfeld. PC-Systeme privater und semiprofessioneller Nutzer werden nicht einbezogen. Damit gliedert sich die Untersuchung in das Tätigkeltsfeld des Investitionsgütermarketing. 
Im Rahmen des Systemkaufs (Hardware, Software und Grundservice) kann der Kunde mit dem Anbieter einen Servicevertrag abschließen, der neben einer ganzen Reihe von Leistungen im Kern vor allem die regelmäßige Systemwartung vorsieht. 57 Für einen regelmäßig zu zahlenden Betrag wird dem Kunden ferner zugesagt, im Fall von Störungen oder Ausfällen eine kurzfristige Instandsetzung der Anlage zu gewährleisten, wobei der wesentliche Anteil aller Material- und Technikerkosten durch den Vertrag bereits abgegolten ist.

Die wesentlichen Vorteile dieser Verträge bestehen für die Kundendienstanbieter zum einen darin, sicher vorhersehbare Kundendienstumsätze zu erzielen. Zum anderen wird eine Präsenz des Kundendienstes beim Kunden nicht mehr zwangsläufig nur mit defekten Geräten und somit schlechter Produktqualität in Verbindung gebracht. Vielmehr gewährleistet die kontinuierliche Betreuung der Hauptleistung durch das Kundendienstpersonal ein Vertrauensverhältnis zwischen Hersteller und Kunden. Der Techniker tritt nicht immer nur bei Negativereignissen in Erscheinung, sondern er wartet und pflegt auch das Computersystem. Damit erhöht er dessen Funktions- und Wirkungsgrad. Für die Nutzer von Computersystemen dagegen werden die laufenden Kosten ihres EDV-Betriebs kalkulierbar. Ein besonderes finanzielles Risiko, verursacht durch Systemausfälle und -störungen, besteht kaum noch.

Mit Hilfe derartiger Verträge erzielte die Branche in den vergangenen Jahren einen erheblichen Anteil ihrer gesamten Kundendienstumsätze. 58 In der letzten Zeit dokumentiert sich jedoch in den verschiedenen Systemmärkten - Großrechnersysteme, mittlere proprietary Systeme, mittlere offene Systeme und Personal Computer - ein zunehmend anderes Serviceverhalten der Nachfrager. ${ }^{59}$ Eine

57 Mit Systemgeschäften werden im Investitionsgütermarketing Kaufpakete bezeichnet, die aus mehreren voneinander abhängigen Teilsystemen oder -komponenten bestehen. Mit der Ausgangsinvestition erfolgt eine Festlegung auf einen Systemtyp und damit eine "Systemarchitektur", die für Folgekäufe eine Bindewirkung hat. Zum Marketing in Systemgeschäften vgl. u.a. Backhaus, K., Investitionsgütermarketing, a.a.O., S. $318 \mathrm{ff}$.

58 Vgl. Nixdorf Computer AG, Kundendienst - Kaufmännischer Produktbericht 6/89, Paderborn 1989, S. 1.

59 Großrechnersysteme, auch häufig als Mainframe-Computer bezeichnet, sind derzeit die leistungsstärksten Mehr-Platz-Computer. Dabei greifen eine Vielzahl verschiedener, dezentraler Bildschirm-Arbeitsplätze aư eine zentrale Systemeinheit zurück, die Anwendungssoftware zur Verfügung stellt. Mittlere Systeme stellen die nächst kleinere Leistungsstufe an Mehr-Platz-Computern dar. Während "proprietary" Systeme dabei mit einer herstellerspezifischen Betriebssystem-Software gesteuert werden, arbeiten "offene" Systeme mit etablierten Betriebssystem-Standards wie z.B. Unix. Personal Computer sind selbständige Ein-Platz-Sy- 
der Hauptursachen liegt dabei in den unterschiedlichen Voraussetzungen und Rahmenbedingungen, die den Produkt- und Servicewettbewerb in diesen Teilmärkten kennzeichnen (vgl. hierzu und im folgenden Abbildung 5).

Der Großrechnermarkt hat den größten Bedarf an klassischen TKD-Leistungen (TKD = Technischer Kundendienst) und aufgrund massiver Technologiebarrieren damit zugleich auch die höchste Vetragsdurchdringungsrate. Dies bewirkt eine sehr enge Bindung an den Hauptleistungs-Hersteller. Daher bestehen bislang kaum Möglichkeiten für einen aufkommenden Servicewettbewerb. Bei mittleren proprietary und mittleren offenen Systemen ist dieses Bild schwächer ausgeprägt, doch ähnlich strukturiert.

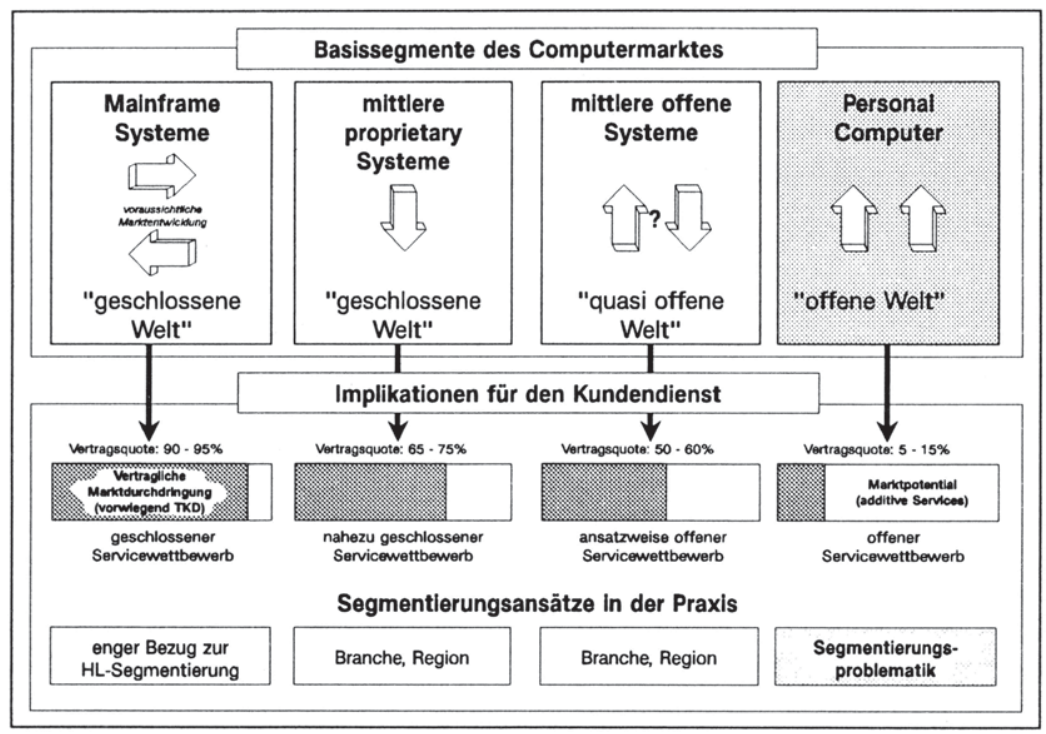

Abbildung. 5: Basissegmente des Computermarktes

Ein tatsächlich offener Kundendienstwettbewerb besteht nur im PC-Markt. Dieser Markt unterlag im Laufe der letzten Jahre den größten Veränderungen. Einige der herausragenden Entwicklungen sind dabei:

steme mit eigenem Betriebssystem und eigener Anwendungssoftware. Eine Vernetzung mehrerer PCs (PC-Netz), und damit ein Mehrplatzbetrieb, ist möglich. 
- rapide Entwicklungssprünge in der Hardwaretechnologie, 60

- $\quad$ in auf breiter Basis akzeptierter Betriebssystemstandard mit MS-Dos, 61

- fortschreitender Ausbau von Schnittstellen zu anderen Betriebssystemen (z.B. Integration Apple/Dos), 62

- ein fast unüberschaubar breites, qualitativ hochwertiges Angebot an Anwendungssoftware,

- hohe Anwenderfreundlichkeit durch immer einfacher werdende Bedienung mittels grafischer Benutzeroberflächen,

- ansteigende Kompatibilitäten zwischen Hardware- und Softwareprodukten und nicht zuletzt

- ein hoher Preisverfall bei Hardwarekomponenten durch den Markteintritt vieler No-Name-Anbieter. 63

Dementsprechend vielfältig gestaltet sich auch der PC-Servicewettbewerb. Angesichts zunehmend "ausfallsicherer Rechner" entsprechen traditionelle Kundendienstverträge kaum noch heutigen Anforderungen. Bei vielen Nachfragern hat sich daher Skepsis hinsichtlich des Nutzens einer umfassenden und verhältnismäßig teueren Ausfallprävention aufgebaut. Insofern gilt es innovative, kundengerechte Dienstleistungsformen zu finden, die den jeweils spezifischen Anforderungen entsprechen.

Bedingt durch die Eigenarten der PC-Technologie ergeben sich in diesem Bereich besondere Schwierigkeiten. In den mehr oder weniger geschlossenen Computermarktsegmenten, ist es relativ einfach, die kundendienstspezifische Bedürfnissituation aus der jeweiligen Einsatzsituation der Hauptleistung - der Computeranlage - abzuleiten. Die Individualität von Großrechnerlösungen oder der starke Branchenbezug mittlerer Systeme bestimmen vielfach direkt die servicebezogenen Anforderungen. Kundendienstsegmente sind somit für eine Ser-

60 Vgl. o.V., Windows stărkt die Nachfrage, In: Chlp, 1992, Nr. 1, S. 14;

61 Vgl. o.V., Barometer Prognose für Betriebssysteme - Dos blelbt Spltze, in: Chip, 1992, Nr. 2 , S. 14.

62 Vgl. o.V., Bus-Produkte stark gefragt, In: Chlp, 1991, Nr. 10, S. 13.

63 Vgl. o.V., PC-Markt in Deutschland, Vobis ist spitze, in: Chip, 1991, Nr. 5, S. 14. 
viceorganisation in diesen Teilmärkten anhand relativ einfacher Faktoren (z.B. Anlagentyp, Branche, Region) zu klassifizieren.

Im PC-Markt ist diese Aufgabe wesentlich komplexer. Obwohl Kunden hier häufig gleiche PC-Konfigurationen (Hardware, Software) betreiben, ist davon auszugehen, daß dennoch gänzlich verschiedene Dienstleistungsbedürfnisse bestehen. Ursächlich hierfür können einerseits unterschiedliche Anwendungsvoraussetzungen und -fähigkeiten sein. Andererseits sind verschiedene Einbindungstiefen der Systeme in den Aufgaben- und Lösungsprozeß der Unternehmung einzubeziehen.

\section{Zlel und Gang der Untersuchung}

Zielsetzung der vorliegenden Untersuchung ist die Entwicklung eines spezifischen Segmentierungsmodells für investive Kundendienstmärkte. Mit Hilfe eines solchen Konzeptes sollen bedürfnishomogene Gruppen von Servicenachfragern identifiziert werden. Darüber hinaus ist die Machtsituation einzelner Buying Center-Mitglieder beim Leistungskauf zu analysieren, um geeignete Marktbearbeitungsstrategien für die Kundendienstpolitik ableiten zu können.

Voraussetzung für diese Zielsetzung ist zunächst die Operationalisierung der kundendienstspezifischen Bedürfnissituation von Unternehmen. Die Generierung eines derartigen theoretischen Konstruktes bildet deshalb auch den Ausgangspunkt der weiteren Überlegungen.

In einem zweiten Schritt gilt es, die verfahrenstechnischen Grundlagen der Servicesegmentierung zu bilden. Der konzeptionelle Ansatz geht dabei im wesentlichen auf mehrstufige Ansätze der Investitionsgütersegmentierung zurück. Diese Modelle berücksichtigen die im Vergleich zum privaten Kautverhalten komplexeren Strukturen betrieblicher Einkauf- und Entscheidungsprozesse. Um den strategischen Charakter der Marktsegmentierung zu unterstreichen, soll darüber hinaus ein enger Bezug zu Marktbearbeitungsmöglichkeiten geschaffen werden.

Die Identifizierung trennscharfer Segmente im Servicemarkt für kommerzielle Personal Computer stellt neben einer hypothesengestützten Überprüfung der 
konzeptionellen Überlegungen die empirische Zielsetzung der Untersuchung dar.

Mit der beschriebenen Ziel- und Schwerpunktlegung ist bereits der Gang der Untersuchung vorgezeichnet. In Kapitel B werden zunächst die theoretischen und konzeptionellen Grundlagen der Arbeit gelegt. Hierzu werden eingangs zentrale Besonderheiten des Kundendienstes im Rahmen einer strategischen Marktsegmentierung dargestellt. Aufbauend auf einem Literaturüberblick über die wichtigsten mehrstufigen Segmentierungsansätze, wird dann die Eignung bestehender Konzepte für Probleme des Kundendienstmarketing diskutiert (B.1). Daraufhin erfolgt im Rahmen theoriegeleiteter Überlegungen die Operationalisierung des kundendienstbezogenen Kaufverhaltens. Hierzu wird mit Hilfe von Indikatoren (Brückenvariablen) ein Zusammenhang zwischen dem Kundendienstbedarf von Unternehmen und den servicebezogenen Ausgangsvariablen hergestellt (B.2). In Kapitel B.3 wird ein zweistufiges Modell zur Kundendienstsegmentierung entwikkelt. Mit einem Bezugsrahmen und grundlegenden Hypothesen für die weitere empirische Untersuchung schließt der theoriegeleitete Teil dieser Arbeit ab (B.4).

Im Teil C der Untersuchung erfolgt die empirische Analyse zur Segmentierung von Kundendienstleistungen. Ausgehend von einer Beschreibung der Stichprobenstruktur und des Untersuchungsdesigns (C.1) werden zunächst die grundlegenden Annahmen und Voraussetzungen des aufgestellten Segmentierungsansatzes überprüft (C.2). Im Mittelpunkt von Kapitel C.3 steht die Ermittlung kundendienstbezogener Kerndimensionen. Die Bildung, Darstellung und Trennschärfenanalyse von Bedürfnisclustern im Kundendienstmarkt für PCs schließt sich an. Ansatzpunkte für eine zielgruppengerechte Ansprache und Bearbeitung der einzelnen Segmente werden in Kapitel C.4 ermittelt. Hierzu werden jeweils die gruppenspezifischen Machtstrukturen hinsichtlich ihres kundendienstbezogenen Kaufverhaltens untersucht. Die Aussagekraft des Segmentierungsergebnisses wird in Kapitel C.5 diskutiert.

Kapitel D dient schließlich der Formulierung von Implikationen für die Praxis. Es stehen grundsätzliche Möglichkeiten der kundendienstbezogenen Marktbearbeitung zur Diskussion. U.a. erfolgt dabei eine Darstellung grundsätzlich erfolgversprechender Normstrategien im PC-Kundendienstmarkt. Anregungen zu weiteren Forschungsaufgaben im Rahmen der Kundendienstsegmentierung bilden den Abschluß der Arbeit. 


\section{B. Konzeptionelle Grundlagen zur Segmentierung von Kundendienst- leistungen in investiven Märkten}

\section{Ansätze der Marktsegmentierung und ihre Übertragbarkeit auf Kun- dendienstprobleme}

Die Auseinandersetzung mit Serviceleistungen im Sinne von Folgeservices bedingt die Betrachtung eines eigenständigen Marktes. 64 Der enge Hauptleistungsbezug beeinflußt allerdings den Typ dieses Marktes. Im Rahmen der vorliegenden Untersuchung stellt die Hauptleistung ein Investitionsgut dar. Insofern weisen auch diesbezügliche Kundendienstkäufe investive Charakteristika auf. Zentrale Kennzeichen von Investitionsgütermärkten treffen somit auch für die zugrundeliegende Problemstellung zu. Dies betrifft insbesondere das multipersonale Entscheidungsverhalten beim Servicekauf. 65

Unternehmen fällen eine Kaufentscheidung üblicherweise für das System, von dessen Einsatz sie sich angesichts ihrer vorliegenden betrieblichen Aufgabenstellung den größten Nutzen versprechen. Aus kundendienstbezogener Perspektive haben jedoch alle Käufer die Entscheidung über eine Systemarchitektur (hier: PC-Technologie) in der Vergangenheit bereits getroffen, d.h. der zu segmentierende Servicemarkt besteht aus Unternehmen mit gleicher bzw. vergleichbarer Systemphilosophie. 66 Jede Systementscheidung war dabei mit einer feststehenden Nutzenerwartung verknüft.

Die Ausgangssituation bei kundendienstbezogenen Segmentierungsaufgaben stellt sich damit anders dar als bei hauptleistungsbezogenen. In bezug auf ser-

64 Im folgenden sind die Begriffe Kundendienst(-leistung) bzw. Service(-leistung) Immer I.S.v. Folgeservices zu verstehen.

65 Vgl. zu Kennzeichen des Investitionsgütermarketing Backhaus, K., Investitionsgütermarketing, a.a.O., S. 3 ff; Kratz, J., Der Interaktionsprozeß beim Kauf von einzelgefertigten Investitlonsgütern, a.a.O., S.54 f; Kern, E., Der Interaktionsansatz im Investitionsgütermarketing, a.a.O., S. 1 f.

66 Unterschiede ergeben sich vielmehr aufgrund verschiedener Einbindungstiefen der Hauptleistung in den jewelligen Unternehmensprozeß. Dieser Integrationsgrad bestimmt die Abhăngigkelt der Unternehmen von den PC-Systemen. Darüber hinaus sind die Einsatzbedingungen der Produkte, die Kompetenzvoraussetzungen der Anwender und der grundlegende Problemlősungsbezug der verwendeten Software unterschiedlich. Zu diesen Aspekten vgl. Kap. B.2. 
vicebezogene Kaufentscheidungen liegen veränderte Bedürfnisentstehungsprozesse zugrunde.

Erst ab dem Zeitpunkt der Hauptleistungs-Inbetriebnahme wird das Ausmaß des tatsächlichen Nutzens deutlich. Art und Umfang dieses realisierten Nutzens werden zwar in einzelnen Unternehmen stark differieren. Insgesamt wird das Nutzenergebnis jedoch in der überwiegenden Zahl der Fälle nicht den ursprünglichen Vorstellungen entsprechen. Wenngleich sich der verwirklichte Nutzen zwar aufgrund von Einsatzerfahrungen im Laufe der Zeit noch steigern kann, wird das gesamte erwartete Potential vielfach erst durch eine zusätzliche Inanspruchnahme von Services verwirklicht werden können. Die Differenz zwischen tatsächlichem und erwarteten Nutzen (="Nutzendefizit") bestimmt damit in der Konsequenz die servicespezifische Bedürfnissituation von Unternehmen (vgl. hierzu Abbildung 6).

Die Ursachen dieses "Gaps" können vielfältig sein. Hierbei angesprochen sind zunächst Systemausfälle und -störungen sowie Mängel in der Anwendung. Zudem kann eine Änderung situativer Faktoren der Anwender als Auslöser für das Nutzendefizit eintreten (z.B. der Ausfall qualifizierter Anwender). Ferner besteht die Möglichkeit, daß die Käufer ihre Nutzenvorstellungen umdefinieren. Dies betrifft zumeist die Erweiterung gegenwärtiger Anwendungsmöglichkeiten, kann sich aber auch auf eine Ausweitung der ursprünglichen Problemstellung beziehen (z.B. Vernetzung vonEinzelplatz-PCs). Letztlich können aber auch neue Anforderungen in anderen Bereichen entstehen, die in die Problemlösung integriert werden sollen (z.B. zusätzlicher Aufbau einer Kundendatei).

Der Markt für Folgeservices grenzt sich also über die Nutzenlücke bei der Realisation der hauptleistungsverbundenen Nutzenerwartungen gegenüber dem Hauptleistungsmarkt ab. Somit steht im Mittelpunkt der folgenden Segmentierungsüberlegungen ein Markt, der sich durch Nutzenerwartungen als bedürtnisgenerierende Faktoren auszeichnet.

Zur Entwicklung eines kundendienstpolitischen Segmentierungsansatzes ist zunächst eine inhaltliche Einordnung in den größeren Zusammenhang der Segmentierungsforschung vorzunehmen. Darüber hinaus sind Bewertungsmaßstäbe zu entwickeln, um bestehende Konzepte kritisch in bezug auf ihre Übertragbarkeit für Kundendienstprobleme zu überprüfen und entsprechende Veränderungen vorzunehmen. 


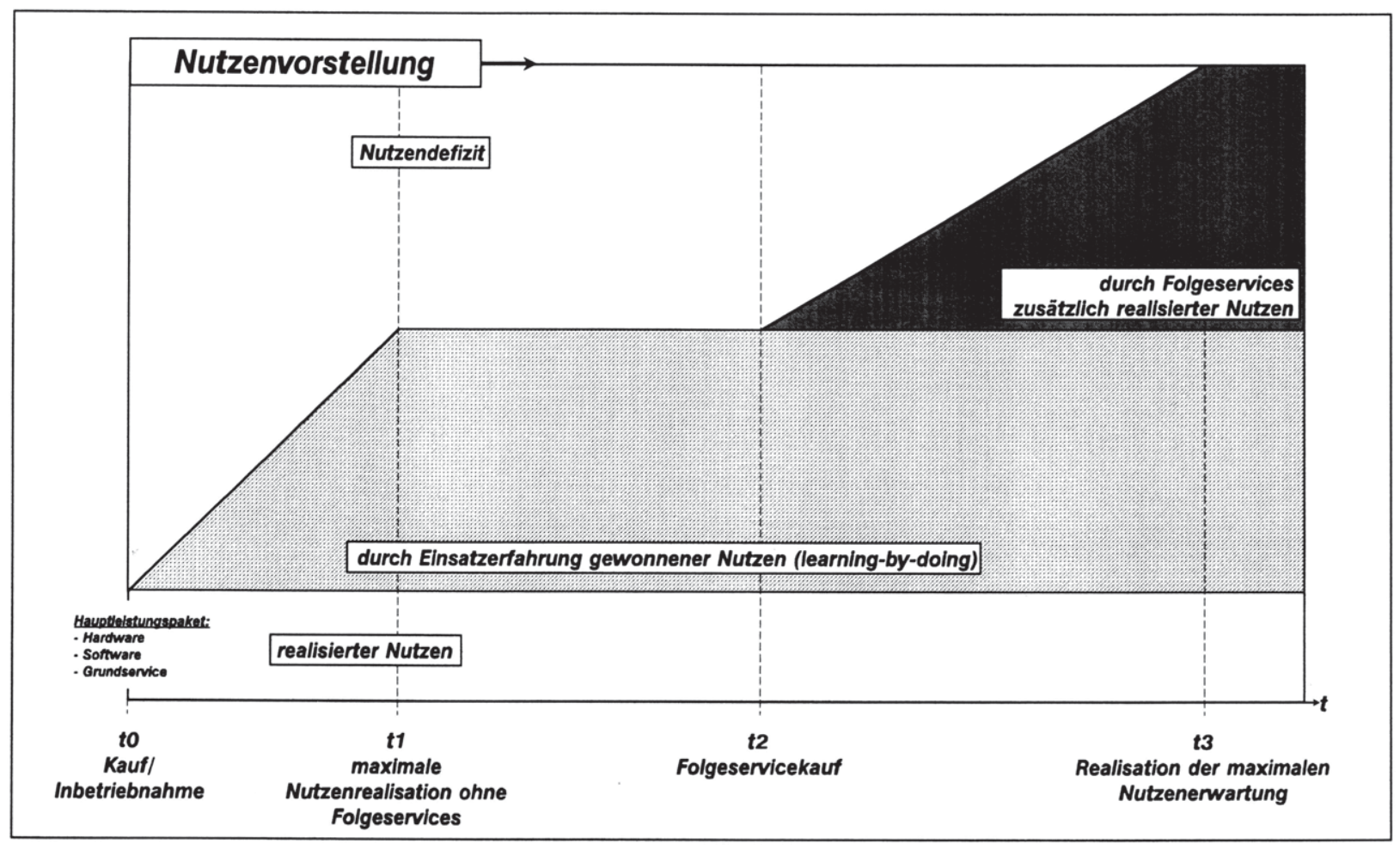

Abbildung 6: Kundendienstbezogene Nutzensituation 


\subsection{Marktsegmentierung im Spannungsfeld der Marketingforschung}

Die Reihe der wissenschaftlichen Veröffentlichungen zur Marktsegmentierung ist lang. Kaum einem anderen Thema hat die Marketingwissenschaft in der Vergangenheit ein vergleichbar intensives Interesse entgegengebracht. Es besteht jedoch keineswegs eine einheitliche Vorstellung bezüglich der Inhalte und Aufgaben der Segmentierung. Besonders in bezug auf die Einordnung in den $\mathrm{Ge}-$ samtprozeß der Marketingplanung lassen sich eine Reihe verschiedener Auffassungen anführen (vgl. Tabelle 1).

\begin{tabular}{|l|l|}
\hline Aufgabe und Wesen der Marktsegmentierung & Autor \\
\hline "Alternative zur Produktdifferenzierung" & Smith (1956) \\
\hline "Methode der Absatzgestaltung", \\
"besondere Methode der Produktdifferenzierung" & Angehrn (1968) \\
\hline "Managementstrategie" & Bass, Tigert, Lonsdale (1968) \\
\hline "zweckorientiertes Ordnungsprinzip" & Baum (1970) \\
\hline "statistisches Verfahren zur Bearbeitung von Daten" & GfK (1971) \\
\hline "Strategie des Marketing", & Winkler (1972) \\
\hline "Methode der Marktforschung" & "Managementphilosophie", \\
"Strategie der Marktanalyse und Marketingplanung" & Sheth (1972) \\
\hline "unternehmenspolitische Konkretisierung einer & Scheuch (1974) \\
\hline "unternehmerischer Marktforschungsprozess" & Hansen (1976) \\
\hline "Methode zur Abgrenzung von Zielgruppen für das Marketing" & Kroeber-Riel (1980) \\
\hline "Marketingstrategie" & Kols (1986) \\
\hline "Prinzip der differenzierten Marktbearbeitung" & Meffert (1986) \\
\hline "Schartschützenkonzept" & Becker (1988) \\
\hline "Identifikation kaufverhaltensgleicher Nachfragergruppen" & Backhaus (1992) \\
\hline ... & \\
\hline
\end{tabular}

Tab.1: Überblick über ausgewählte Auffassungsinhalte der Marktsegmentierung67

67 Vgl. Smith, W.R., Product Differentation and Market Segmentation as Alternative Marketing Strategies, in JoM, Vol. 21, 1956, No. 1, S. 2; Angehrn, O., Marktsegmentierung als Absatzmethode, in: GFM, 14. Jg. 1968, Nr. 2, S. 38; Bass, F.M., Tigent, D.J., Lonsdale, R.T., Market Segmentation: Group versus Individual Behavior, in: Journal of Marketing Research, Vol. 5, 1968, No. 3, S. 265; Baum, J.G., Marktsegmentierung und Marktlücken, in: ZV + ZV, 67. Jg., 
Trotz dieser Vielzahl einzelner Auslegungen sind zwei grundlegende Interpretationsrichtungen festzustellen. 68 Dabei wird die Marktsegmentierung zum einen als Methode der Marktforschung dargestellt, wodurch inr ein eher instrumenteller Charakter zugewiesen wird. Eine zweite Gruppe von Autoren dagegen interpretiert die Marktsegmentierung bereits als Marketingstrategie und erweitert inre analytischen Aufgaben um marktbearbeitungsbezogene Gesichtspunkte.

Verfechter der rein instrumentalen Segmentierungsauffassung rücken die Marktaufteilung in den Mittelpunkt ihrer Betrachtung. 69 Die Zusammenstellung und Auswahl tragfähiger Segmentierungskriterien genauso wie die Entwicklung geeigneter Segmentierungsverfahren stehen im Vordergrund dieser Betrachtungsweise. 70 Dabei vollzieht sich die Strukturierung aller tatsächlichen und potentiellen Nachfrager eines Gutes anhand spezifischer Kriterien, welche die unterschiedlichen Präferenz- und Bedarfsstrukturen des Gesamtmarktes differenziert herausheben. 71 Probleme der Marktbearbeitung werden weitestgehend ausgegrenzt. 72

1970, S. 1243; GfK, Marktsegmentierung - ein Instrument zur Zielgruppenbestimmung, in: GfK-Sonderdienst, 1971, 2. Doppelheft, S. 42; Winkler, A., Praktische Anwendungen der Marktsegmentierung. Marktsegmentierung als Methode der Marktforschung, in: Jahrbuch der Absatz- und Verbrauchsforschung, 1972, Nr. 2, S. 102; Sheth, J., Marktsegmentierung als relevante Planungshilfe des Marketing - Marktsegmentierung als Strategie des Marketing, in: Jahrbuch der Absatz- und Verbrauchsforschung, 1972, Nr. 2, S. 129; Scheuch, F., Logische Struktur und pragmatische Bedeutung der Marktsegmentierung, in: Die Unternehmung, 28. Jg., 1974, Nr. 3, S. 213; Kols, P., Bedarfsorientierte Marktsegmentierung auf Produktivgütermärkten, a.a.O., S. 31; Meffert, H., Marketing, a.a.O., S. 243.; Backhaus, K., Investitionsgütermarketing, a.a.O., S. 130.; Becker, J., Marketing-Konzeption: Grundlagen des strategischen Marketing-Managements, 2. Auff., München 1988, S. 223.

68 Vgl. hierzu und zum folgenden Literaturüberblick: Kols, P., Bedarfsorientierte Marktsegmentierung auf Produktivgütermärkten, a.a.O., S. $24 \mathrm{ff}$.

69 Vgl. hierzu: Ahlert, D., Die Probleme der Abnehmerselektion und der differenzierten Absatzpolitik auf der Grundlage der segmentierenden Markterfassung, in: Der Markt, 1973, Nr. 4, S. 103; Boyd, H.W., Massy, W.F., Marketing Management, New York 1972, S. 87; Lignel, J., Cadet, A., The Problems of Market Segmentation, in: The European Marketing Research Review, Vol. 2, 1967, No. 2, S.24 ff; Böhler, H., Methoden und Modelle der Marktsegmentierung, a.a.O., S. $10 \mathrm{f}$.

70 So definieren Boyd/Massy die Aufgabe der Marktsegmentierung als Prozess in dem ein Unternehmen seinen Markt in Untergruppen oder Teilmärkte gliedert. Boyd, H.W., Massy, W.F., Marketing Management, a.a.O.,S. 84.

71 Innerhalb dieser Gruppe von Ansätzen lassen sich zwei weitere Richtungen unterscheiden: Zum einen befassen sich Untersuchungen mit verhaltenswissenschaftlichen Aspekten. Im Mittelpunkt steht damit die Identifikation und Dokumentation generalisierbarer Unter- 
Die strategische Marktsegmentierung integriert dagegen neben den Aspekten der Marktanalyse zusätzlich Gesichtspunkte der Marktbearbeitung in ihre Betrachtungen. Dabei steht die Ausrichtung des Marketingmix auf die segmentspezifischen Präferenzen von Nachfragern im Vordergrund. 73 Die strategische Marktsegmentierung läßt sich somit als zweistufiger, dynamischer und interdependenter Prozeß darstellen:74

(1) Aufteilung des Gesamtmarktes in möglichst homogene Segmente und

(2) Anpassung der absatzpolitischen Instrumente an die spezifischen Anforderungen der Segmentmitglieder.

Deutlich betont wird hierbei die gegenseitige Abhängigkeit von Marktaufteilung und -bearbeitung. Einerseits gelingt die Bestimmung und Anpassung des Marketingmix um so besser, je höher die Homogenität in einzelnen Segmenten ist. Andererseits kann jedoch ein Bedarfs- und Wertewandel innerhalb einzelner Teilmärkte zu wachsenden Ungleichgewichten zwischen Leistungsangebot und Anforderungen führen. Dies muß in der Konsequenz zu einer Neuaufteilung der

scheidungsmerkmale von Kundengruppen, die Schlußfolgerungen auf ein gruppentypisches Kautverhalten zulassen. Die Marktsegmentierung dient damit in erster Linie der wissenschaftlichen Käuferverhaltensforschung. Vgl. hierzu insbesondere auch: Frank, R.E., Massy, W.F., Wind, Y., Market Segmentation, a.a.O., S. 11 ff. Vertreter einer entscheidungsorlentierten Forschungsrichtung befassen sich mit der Frage, wie aus einer heterogenen Gesamtheit von Nachfragern profitable Zielgruppen herausgefiltert und operational identifiziert werden können. Gegenstand der Betrachtung ist vor allem der effiziente und zieladäquate Mitteleinsatz des absatzpolitischen Instrumentariums. Vgl. hierzu u.a. Bauer, E., Markt-Segmentierung, a.a.O., S. 51.

72 Vgl. hierzu auch Gröne, A., Marktsegmentierung bei Investitionsgütern, a.a.O., S. 30; Bauer, E., Markt-Segmentierung, a.a.O., S. 49; Jacob, R., Marktsegmentierung auf Investitionsgütermärkten als Grundlage der Steuerung absatzpolitischen Mitteleinsatzes. Dargestellt am Beispiel des Lastkraftwagenmarktes, Bern, Frankfurt/M. 1972, S. 16; Winkler, A., Praktische Anwendung der Marktsegmentierung, a.a.O., S. 104 ff; Backhaus, K., Weiber, R., Marktsegmentierungsprobleme in sich verändernden Märkten, in: VDI-Berichte, 1986, Nr. 616, S. 139 ff.

73 Vgl. hierzu auch die ausführliche Zusammenstellung zentraler Segmentierungsansätze des Investitionsgütermarketing von Plank, R.E., A Critical Review of Industrial Market Segmentation, in: Industrial Marketing Management, Vol. 14, 1985, No. 14, S. $79 \mathrm{ff}$.

74 Vgl. Jacob, R., Marktsegmentierung auf Investitionsgütermärkten, a.a.O., S. 17. Vergleichbare Darstellungen finden sich auch bei Wilkie, W.L., The "Product Stream" of Market Segmentation. A Research Approach, in: Relevance in Marketing. Marketing in Motion. Combined Proceedings of the Spring and Fall Conferences 1971, Hrsg.: Allvine, F.C., Chicago 1972, S. 317. 
Segmente und damit auch zu einer Anpassung des Marketinginstrumentariums führen.

Zudem wird der dynamische Charakter der strategischen Marktsegmentierung unterstrichen. Sie ist keine einmalige, statische Bestandsaufnahme. ${ }^{75}$ Vielmehr handelt es sich um eine permanente Kontrolle und Beobachtung gegliederter Märkte mit dem Bestreben, unterschiedliche Anforderungsprofile von potentiellen und aktuellen Nachfragern mit spezifischen Marketingprogrammen abzustimmen.

Ein Segmentierungskonzept für Kundendienstleistungen ordnet sich der Auslegung einer strategischen Marktsegmentierung unter. Entsprechend der gewählten Zielsetzung wird damit der Auffassung gefolgt, daß die Aufteilung von Servicemärkten nicht als Selbstzweck zu betrachten ist, sondern vielmehr Ansatzpunkte zur Marktbearbeitung von Kundendienstsegmenten aufzeigen muß.

Neben der grundsätzlichen Ausrichtung des Forschungsschwerpunktes ist es ferner erforderlich, einen einheitlichen Maßstab zur Beurteilung von Segmentlerungsansätzen heranzuziehen. Erst hierdurch wird ein Vergleich verschiedener Konzepte sinnvoll möglich. Diesbezüglich sind in der wissenschaftlichen Segmentierungsliteratur eine Reihe von Anforderungen an die Segmentierung und deren Ausgangsvariablen gestellt worden. Die folgenden sechs Gesichtspunkte haben dabei allgemeine Anerkennung zur Beurteilung von Segmentierungskriterien gewonnen und sind von daher auch für kundendienstbezogene Aufgabenstellungen heranzuziehen:76

(1) Mit der Kaufverhaltensrelevanz wird vor allem die Forderung erhoben, daß Segmentierungskriterien einen Bezug zu den Bestimmungsfaktoren des Kaufverhaltens autweisen müssen. Diese Bedingung basiert auf dem originären Zweck der Marktsegmentierung, Märkte in kautverhaltenshomogene Untergruppen einzuteilen bzw. kautverhaltensheterogene Gruppen zu trennen. Bei kundendienstspezifischen Segmentierungsansātzen

75 Vgl. Gröne, A., Marktsegmentierung bel Investitionsgütern, a.a.O., S. 33.

76 Vgi. hierzu auch: Gröne, A., Marktsegmentierung bei Investitionsgütern, a.a.O., S. 43 ff.; Freter, H., Marktsegmentierung, a.a.O., S. 43 ff.; Horst, B., Ein mehrdimensionaler Ansatz zur Segmentierung von Investitionsgütermärkten, a.a.O., S. 131 ff.; Backhaus, K., Investitionsgütermarketing, a.a.O., S. 130 f.; Meffert, H., Marketing, a.a.O., S. 244 f. 
sind darüber hinaus besonders bedürfnisbezogene Aspekte in die $\mathrm{Be}$ trachtung einzubeziehen.

(2) Die Segmentierungskriterien, und damit letztlich der Verhaltensbezug einzelner Segmente, müssen mit vorhan-denen Methoden der Marktforschung erfaßbar und meßbar sein.

(3) Die herangezogenen Segmentierungsmerkmale müssen zeitliche Stabilität aufweisen und über einen zumindest mittelfristigen Zeitraum aussagefähig bleiben. Der gesamte Prozeß der strategischen Segmentierung (Datenerhebung, Analyse, Entwicklung von Marktbearbeitungsprogrammen) beansprucht Zeit. Damit die entwickelten Konzepte letztendlich für die einzelnen Marktsegmente noch aussagekräftig sind, muß gewährleistet sein, daß die anfangs analysierte Segmentsituation zumindest mittelfristig stabil bleibt.

(4) Die Kosten der Marktsegmentierung (Informationsgewinnungs- und auswertungskosten, Kosten der differenzierten Zielgruppenpolitik) müssen in einem vertretbaren Verhältnis zum Segmentierungsnutzen (verbesserte Erlös- und Marktsituation aufgrund einer zielgruppengerechten Marktbearbeitung) stehen.

(5) Die Segmentierungskriterien sollen die Ansprechbarkeit der einzelnen Marktsegmente ermöglichen. Erst hierdurch wird die Vorausetzung dafür geschaffen, daß der Leistungsanbieter die einzelnen Ziegruppen mit spezifischen Programmen auch tatsächlich erreicht.

(6) Mit Hilfe der zielgruppengerechten Gestaltung des Marketing-Instrumenteeinsatzes soll die Effizienz der Marktbearbeitung erhöht werden. Segmentierungskriterien müssen daher Ansatzpunkte für die zielgruppengerechte Ausarbeitung aufzeigen um ungewollte Streuverluste der Marketingmittel zu minimieren.

Aufgrund der vorliegenden investiven Ausrichtung sind Segmentierungsansätze neben den dargestellten Bewertungsmaßstäben zur Kriterienbewertung auch hinsichtlich ihrer konzeptionellen Eignung zu überprüfen. So bedingt das im Vergleich zum Konsumentenverhalten wesentlich komplexere, multipersonale 
Kaufverhalten von Unternehmen bei der Segmentierung einen differenzierten, schrittweisen Filterungsprozeß.77

Aufgrund der Multipersonalität bei betrieblichen Kaufprozessen ist auch für einen servicebezogenen Segmentierungsansatz eine mehrstufige Konzeption erforderlich. Ein solcher Ansatz muß dabei einerseits die Identifizierung der kundendienstspezifischen Bedürfnissituation eines Unternehmens bzw. der maßgeblich prägenden Personen ermöglichen. Andererseits soll er zu einer verbesserten Transparenz in servicebezogenen Entscheidungsprozessen führen und damit den Wandel latenter Bedürfnisse in konkrete Kundendienstnachfrage abbilden.

\subsection{Mehrstufige Segmentierungsansätze}

Mehrstufige Segmentierungsansätze berücksichtigen im Gegensatz zu einstufigen Konzepten 78 in abgestuften Filterungsprozessen inbesondere die multipersonalen Einflußfaktoren des betrieblichen Kaufverhaltens im Segmentierungsprozeß. 79 Damit wird bereits deutlich, daß diese Vorschläge in erster Linie für die Segmentierung von Investitionsgütermärkten entwickelt wurden.

Stellvertretend für die Arbeiten zu diesem Komplex werden im folgenden die Segmentierungsansätze von Wind/Cardozo und Gröne diskutiert und im Hin-

77 Vgl. Backhaus, K., Investitionsgütermarketing, a.a.O., S. 131.

$78 \mathrm{Zu}$ einem Überblick der einstufigen Segmentierung, insbesondere zu verhaltens- und verwendungszweckorientienten Ansätzen vgl. u.a.: Meffert, H., Marketing, a.a.O., S. 245 ff.; Freter, H., Marktsegmentierung, a.a.O., S. 49 ff.; Backhaus, K., Investitionsgütermarketing, a.a.O., S. 132 ff.; Kols, P., Bedarfsorientierte Marktsegmentierung aư Produktivgütermärkten, a.a.O., S. 32 ff.; Bauer, E., Markt-Segmentierung, a.a.O., S. 52 ff.; Frank, R.E., Massy, W.F., Wind, Y., Market-Segmentation, a.a.O., S. 26 ff.; Boyd, H.W., Massy, W.F., Marketing Management, a.a.O., S.89 ff.; Yankelovich, D., Neue Kriterien der Marktsegmentierung, in: Marketing-Management und Organisation, Hrsg.: Britt, S.H., Boyd, H.W., München 1971, S. 209 ff.; Haley, R.I., Benefit Segmentation: A Decisonoriented Research Tool, in: JoM, Bd. 32, 32.Jg., 1968, Nr. 7, S. 30 ff.; Speckmann, R.E., Segmenting Buyers in Different Types of Organizations, in: Industrial Marketing Management, Vol. 10, 1981, No. 10, S. 43 ff.; Wilson, D.T., Industrial Buyers Decision-Making Styles, in: Journal of Marketing Research, Vol. 8 , 1971, Nr. 4, S. 433 ff.; Barnett, N.L., Beyond market segmentation, in: HBR, Vol. 47, JanuaryFebruary 1969, S. 152 ff.; Wilkie, W.L., The "Product Stream" of Market Segmentation, a.a.O., S. $317 \mathrm{ff}$.

79 Vgl. Backhaus, K., Investitionsgütermarketing, a.a.O., S. 134. 
blick auf die Übertragbarkeit für Kundendienstprobleme überprüft. ${ }^{80}$ Beide Ansätze stellen die mehrstufigen Prozesse umfassend dar und sind im Rahmen der Investitionsgütersegmentierung richtungsweisend.

\section{(1) Der zweistufige Segmentierungsansatz von Wind/Cardozo}

Das 1974 von Wind und Cardozo entwickelte Modell kann als Basismodell der mehrstufigen Segmentierung angesehen werden. ${ }^{81}$ Es besteht aus zwei Stufen, wobei die erste durch die Makro- und die zweite durch die Mikrosegmentierung gebildet wird. 82 Die Mikroebene ist weiter unterteilt in eine Prozeß- und Struktursowie eine Individualanalyse (vgl. Abbildung 7). 83

Auf der ersten Stufe werden Unternehmen anhand inrer organisationsbezogenen Charakteristika und ihrer spezifischen Kaufsituation zu Gruppen zusammengefaßt. Typische Merkmale hierfür sind z.B.:

- Unternehmensgröße

- Branche

- Organisationsstruktur

- Standort

- Anwendungs- und Einsatzbereich des Produktes

- Verwendungshäufigkeit

80 Zu einem Überblick über die wichtigsten anglo-amerikanischen Ansätze zur Investitionsgütersegmentierung vgl. Plank, R.E., A Critical Review of Industrial Market Segmentation, a.a.O., S. 81. Auf eine Diskussion mehrdimensionaler Ansätze zur Marktsegmentierung wird an dieser Stelle ganz verzichtet. Vgl. hierzu Backhaus, K., Investitionsgütermarketing, a.a.O., S. 139 ff; Horst, B., Ein mehrdimensionaler Ansatz zur Segmentierung von Investitlonsgütermärkten, a.a.O., sowie die dort angegebene Literatur.

81 Vgl. Strothmann, K.-H., Kliche, M., Marktsegmentierung für High-Tech-Anbieter, in: Marktforschung \& Management, 1989, Nr. 3, S. 82.

82 Auch Jacob stellte, bereits zwel Jahre zuvor ein zweistufiges Segmentierungsmodell vor. Zu Recht wies er auf die zu starke Konsumgüterorientierung der bis dato bestehenden Konzepte hin. In seinem Vorschlag differenziert er eine erste Aufteilung von Produktivgütermärkten I.S.e. Basissegmentierung, an die sich eine zweite, verhaltensorientierte Verfeinerung der Segmente anschließt. Vgl. hierzu Jacob, R., Marktsegmentierung auf Investitionsgütermärkten als Grundlage zur Steuerung absatzpolitischen Mitteleinsatzes, a.a.O.; Derselbe, Gezielte Investgüter-Politik für Marktsegmente, In: Marketing Journal, 1973, Nr. 6, S. 476-479.

83 Vgl. Wind, Y., Cardozo, R., Industrial Market Segmentation, in: Industrial Marketing Management, Vol. 3, Okt. 1974, No. 1, S. 156. 


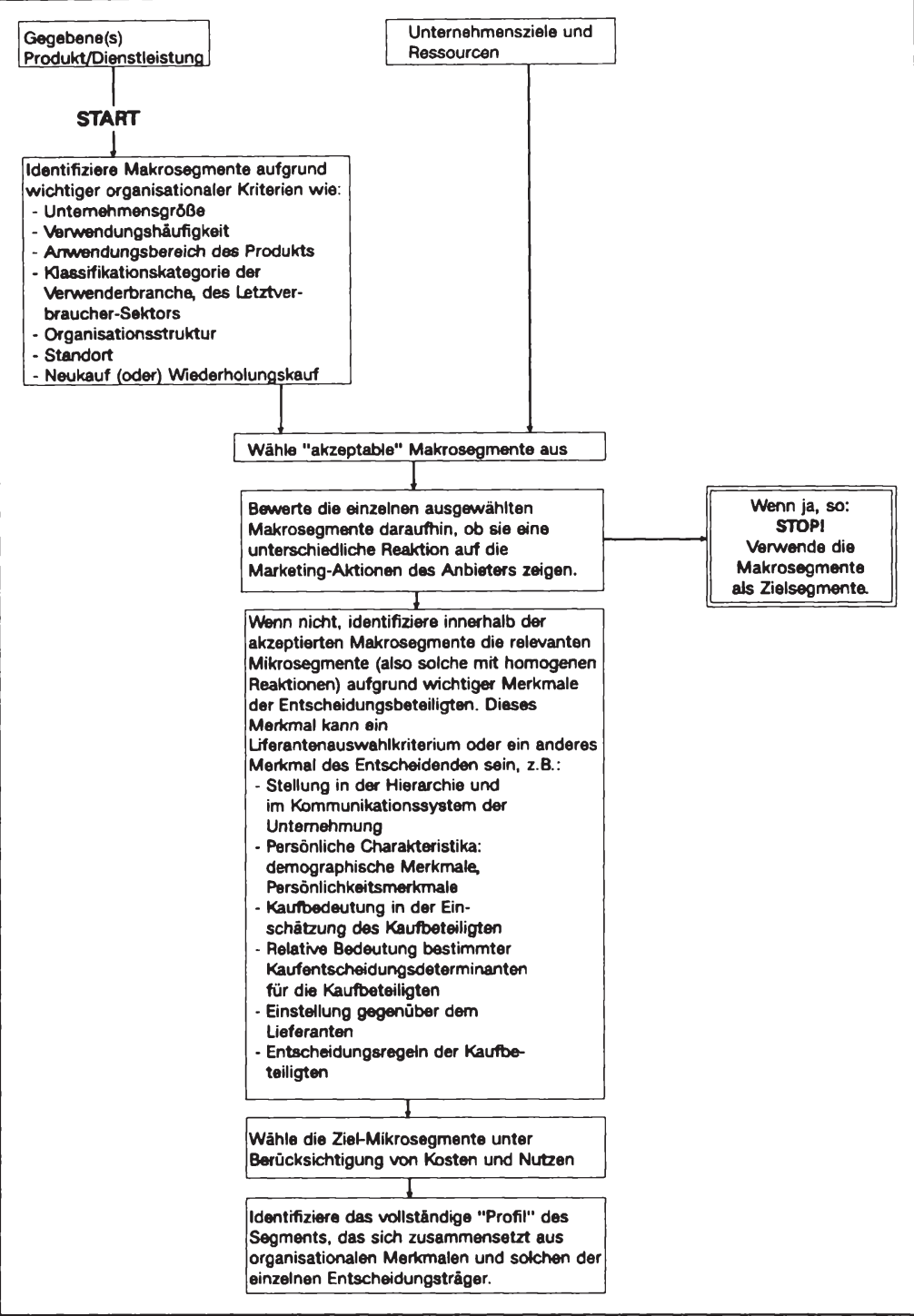

Abbildung 7: Ablaufschema der zweistufigen Marktsegmentierung nach Wind/Cardozo

Quelle:

Übersetzung von Wind, Y., Cardozo, R., Industrial Market

Segmentation, a.a.O., S. 156. 
Weisen die gebildeten Gruppen einheitliche Reaktionsmuster auf die Marketingstimuli von Anbietern auf, empfehlen Wind/ Cardozo den Abbruch des Prozesses, da das Segmentierungsziel erreicht ist.

In der Regel bezieht sich die Homogenität der einzelnen Cluster jedoch lediglich auf einige der angeführten Makromerkmale, wie z.B. Branche, Größe etc. Dabei zeigen aber viele Unternehmen, trotz einer einheitlichen Branchenzugehörigkeit, gänzlich unterschiedliche Kommunikationsgewohnheiten, differenzieren sich in ihren Produktpräferenzen, wählen andere Distributionskanäle und weisen verschiedene Preissensibilitäten auf. Ein zielgruppengerechtes Marketing auf einer solchen Segmentierungsgrundlage ist deshalb kaum möglich.

Somit sind die Makrosegmente weiter zu disaggregieren. Hierzu werden in der zweiten Prozeßstufe zunächst die betrieblichen Einkaufsstrukturen und das Kaufentscheidungsverhalten untersucht (Prozeß- und Strukturanalyse). Geeignete Merkmale dafür sind :

- Merkmale des Einkaufverhaltens

Z.B. Entscheidungsdauer, -autonomie, -regeln, Einstellung gegenüber Lieferanten u.a.

- Merkmale des Buying Centers

Z.B. Größe und Zusammensetzung des Buying Centers, Entscheidungs-, Beeinflussungs- und Kaufanregungsstrukturen, Grad externer Einflußnahme u.a.

Wenn die wesentlichen Strukturen unternehmensbezogener Kaufprozesse offengelegt sind, ist es möglich die Personen zu identifizieren, die diese Prozesse dominieren, d.h. maßgeblich zur Entscheidungsfindung und -durchsetzung beitragen. Es wird angenommen, daß diese Personen als Adressaten jeder kommunikativen Maßnahme geeignet sind. Auf diese Weise erfolgt eine Eingrenzung des Analysebereichs für weitere Stufen. Mit den Zielpersonen wird dann eine Individualanalyse durchgeführt. ${ }^{84}$ Grundlage hierfür sind in erster Linie demographische und psychographische Merkmale 85 .

84 Wind/Cardozo betonen jedoch deutlich, daß die konkrete Auswahl von Segmentierungskriterien vom jeweiligen Untersuchungsfall abhängig ist. Vgl. hierzu Hammann, P., Marktseg- 
Im Anschluß an die erfolgte Segmentierung ist ein vollständiges Profil aller Segmente unter Verwendung der Makro- und Mikrokriterien anzufertigen. Wind/Cardozo betrachten dies als tragfähige Grundlage zur Entwicklung eines zielgruppengerechten Marketingmix.

Zum Modell von Wind/Cardozo sind jedoch auch kritische Einwände anzumerken. So führt eine Aufteilung des Gesamtmarktes anhand direkt beobachtbarer, äußerer Merkmale im Rahmen der Makrosegmentierung häufig zu einer zu groben Aufteilung und bedarf daher einer Weiterentwicklung. Zudem erscheint es problematisch, die postulierten homogenen Reaktionen der Nachfrager in der ersten Stufe empirisch zu belegen. Diese wären fundiert nur in einem Test im Rahmen eines Feldversuchs festzustellen. Ein solches Vorgehen ist aber als Prüfstufe innerhalb eines Segmentierungsprozesses extrem aufwendig und würde in der Konsequenz zu einer erheblichen Verzögerung des gesamten Segmentierungvorgangs führen. 86 Gerade im Bereich des Kundendienstes sind aber die Entscheidungszeiträume eher kurzfristig, so daß nach einer Überprüfung der makroanalytischen Ergebnisse bereits eine vollständige Änderung der Rahmenbedingungen (hier: der Bedarf an Services) vorliegen kann.

Die Individualanalyse erfolgt im Rahmen der Mikroanalyse anhand demographischer und psychographischer Kriterien. Einen weitaus stärkeren Verhaltensbezug würde hierbei jedoch eine zusätzliche Berücksichtigung marketingbezogener "Reaktionskoeffizienten" gewährleisten. 87 Diese dokumentieren individuelle Reaktionen befragter Zielpersonen in bezug auf produkt-, kommunikations-, distributions- und kontrahierungsbezogenen Marketingimpulse von Anbietern. Die Erfassung reaktiver Verhaltensaussagen der Nachfrager zu den unterschiedlichen Mixbereichen kann dabei zur Ableitung gruppenspezifischer "Reaktionsmuster" genutzt werden. Der große Vorteil reaktionsverhaltensbezogener Kriterien ist, daß Segmentierungsergebnisse, die mit Hilfe solcher Merkmale gewonnen wurden, in der Regel unmittelbar erkennbare Ansatzpunkte für

mentierung, in: Technischer Vertrieb, Hrsg.: Plinke, W., Projektgruppe Technischer Vertrieb, Freie Universitaat Berlin, Berlin 1985, S. 1 ff.

Vgl. hierzu auch die Kriterien in Abbildung 7.

Vgl. Engelhardt, W.H., Günter, B., Investitionsgütermarketing, Stuttgart u.a. 1981., S. 91.

Vgl. Freter, H., Marktsegmentierung, a.a.O., S. 45. 
die zielgruppengerechte Verteilung der Marketing-Mittel aufweisen. 88 Von daher ist eine Berücksichtigung solcher Reaktionskoeffizienten auch bei kundendienstbezogenen Segmentierungsaufgaben zu empfehlen.

Ein weiterer Einwand bezieht sich auf methodische Aspekte. Im Mittelpunkt steht dabei die Disaggregation der Makrosegmente, d.h. der Übergang von der Makro- auf die Mikroebene. Wind/Cardozo schlagen nach der Tragfähigkeitsprüfung der ersten Segmentierungsstufe eine weitere Verfeinerung der Makrosegmente vor. Sie berücksichtigen jedoch nicht, daß u.U. nach einer weiteren Aufspaltung, einzelne Mikrosegmente unterschiedlicher Makrogruppen ein sehr homogenes Bild aufweisen können (inter-homogener Zustand). Dies würde in der Konsequenz wiederum zu einem Aggregationsbedarf führen (vgl. Abb. 8).

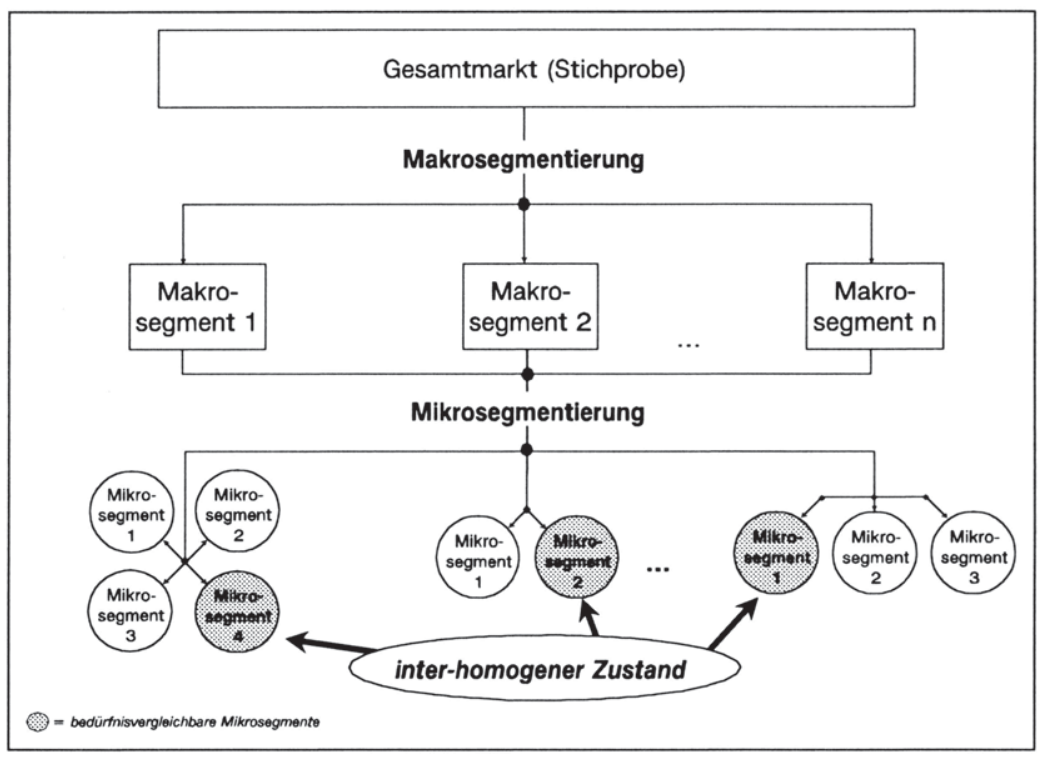

Abbildung 8: Aggregationsproblematik des Segmentierungsansatzes von Wind/Cardozo

88 Boyd/Massy stellen in diesem Zusammenhang fest, daß es grundsätzlich möglich sein muß, aus den jeweiligen Gruppen spezifische Kennzeichen zu extrahieren. Diese können eine geeignete Basis für die zielgruppengerechte Bearbeitung unterschiedlicher Segmente darstellen. Vgl. Boyd, H.W., Massy, W.F., Marketing Management, a.a.O., S. 89. 
Gerade für Kundendienstleistungen trifft dieser Aspekt in besonderem Maße zu, da die Bedürfnislage für Services vielfach unabhängig von Branchenzugehörigkeit und anderen Unternehmensdemographika ist. 89 So kann z.B. ein gleichartiger Bedarf an Schulungsleistungen im Textverarbeitungsbereich sowohl bei Handwerksbetrieben (Makrosegment i) als auch bei Großkonzernen (Makrosegment j) vorliegen.

Schließlich führt das sehr differenzierte konzeptionelle Vorgehen auch bei kundendienstbezogenen Aufgabenstellungen dazu, daß die Datenerhebung bei jedem einzelnen betrachteten Unternehmen sehr aufwendig und komplex ist. Insofern entsteht aus Kostengründen das Bestreben, bei empirischen Untersuchungen eher kleine Stichproben zu erheben. Hieraus ergibt sich andererseits jedoch wiederum die Gefahr, bei der Unterteilung von Makroclustern zu kleine und damit nicht mehr generalisierbare (Mikro-)Segmentgrößen zu gewinnen.

Insgesamt zeigt sich bei diesem Modell ein beträchtlicher Anpassungsbedarf bei einer Anwendung im Bereich der Kundendienstleistungen. Zwar erscheint es möglich, die zweite Modellstufe mit der Analyse der Kaufstrukturen direkt zu übertragen. Die Schwerfälligkeit der Makroanalyse mit der zugeordneten Prüfphase sowie die durch die Unterordnung der Mikroanalyse unter die Ergebnisse der Makroanalyse ausgelösten Inter-Homogenitäten kennzeichnen jedoch den Änderungsbedarf des Modells von Wind/Cardozo.

(1) Der dreistufige Segmentierungsansatz von Gröne

Der dreistufige Segmentierungsansatz für Investitionsgütermärkte von Grōne stellt gegenüber zweistufigen Konzepten vor allem hinsichtlich einer übersichtlicheren und klareren Systematisierung der Segmentierungskriterien eine Weiterentwicklung dar. 90

89 Vgl. Kap. A.2.3, insb. Abbildung 4.

90 Eigentlich entwickelt Gröne ein vierstufiges Modell. Er schließt jedoch seine erste Ebene, die Ebene der Umwelt, aus seiner weiteren Untersuchung unmittelbar wieder aus. Grōne begründet dies folgendermaßen: "Über eine Typologisierung von Umweltkonstellationen lassen sich im Rahmen nationaler Märkte zwangsläufig nur sehr allgemeine, wenig aussagefähige und differenzierende hypothetische Bezüge im industriellen Einkautsverhalten herstellen. Insofern führt eine U-Segmentierung absehbar zur Abgrenzung von lediglich grob bezeichne- 
Gröne bildet im Rahmen seines Modells einen verhaltenswissenschaftlichen Schwerpunkt. 91 Er versucht, alle Faktoren, die möglicherweise Einfluß auf den Verlauf und den Ausgang betrieblicher Kaufentscheidungsprozesse haben, in einem mehrstufigen Modell zusammenzustellen.92 Die eigentliche Segmentierung besteht aus drei Stufen deren Segmentierungsvariablen jeweils aus unterschiedlichen Merkmalskategorien entnommen werden. Ursprünglich umfaßt Grönes Modell sogar vier Merkmalskategorien, mit deren Hilfe er versucht, eine Typologisierung des organisationalen Kaufverhaltens zu entwickeln 93 :

I. Ebene der Umwelt (U-Ebene)

/keine funktionale Berücksichtigung im Modell

II. Ebene der Organisation (O-Ebene)

- organisationsdemographische Merkmale

- Aufbauorganisation im Einkauf

- Ablauforganisation im Einkauf

III. Ebene des Kollektivs (K-Ebene)

- gruppendemographische Merkmale

- interpersonelle Beziehungen

IV. Ebene des Individuums (I-Ebene)

- soziodemographische Merkmale

- verhaltensbezogene Merkmale

Von besonderer Bedeutung ist bei diesem Modell die systematische Prüfung aller angeführten Merkmale bzgl. ihrer Kaufverhaltensrelevanz. Da hierbei eine direkte Verbindung zwischen der tatsächlichen Kaufwahrscheinlichkeit eines Ab-

ten Verhaltenssegmenten, in denen bei näherer Betrachtung eine Vielzahl von heterogenen, spezifischen Kaufverhaltensweisen erkennbar werden." Gröne, A., Marktsegmentierung bei Investitionsgütern, a.a.O., S. 35.

91 Ein in hohem Maße ähnlicher Segmentierungvorschlag, auf den an dieser Stelle nicht weiter eingegangen werden soll, wurde von Scheuch entwickelt. Im Gegensatz zu Gröne legt Scheuch bei der Wahl seiner Segmentierungskriterien den Schwerpunkt auf "die Charakteristik der Abnehmer als beschaffende Organisationen und die relative Bedeutung der Beschaffungsobjekte für die Organisationen...". Vgl. hierzu Scheuch, F., Investitionsgüter-Marketing, Opladen 1975, S. $219 \mathrm{ff}$.

92 Vgl. Gröne, A., Marktsegmentierung bei Investitionsgütern, a.a.O., S. 26

93 Vgl. Ebenda, S. 18 
nehmers (abhängige Variable) und den Segmentierungskriterien (unabhängige Variablen) jedoch nicht hergestellt werden kann, entwickelt Gröne ein Brückenkonstrukt. Dabei operationalisiert er diesen Zusammenhang durch Einführung von Indikatoren, sog. "Brückenvariablen" (vgl. Abb. 9). ${ }^{94}$ Allerdings nutzt er die Indikatoren nicht für die Segmentierung, sondern lediglich im Rahmen einer Verhaltensrelevanzprüfung.

Letztendlich kommt Gröne zu dem Ergebnis, daß zwar eine Reihe von Variablen erheblich zum Verständnis des industriellen Kaufverhaltens beitragen, so z.B. zur Erklärung von

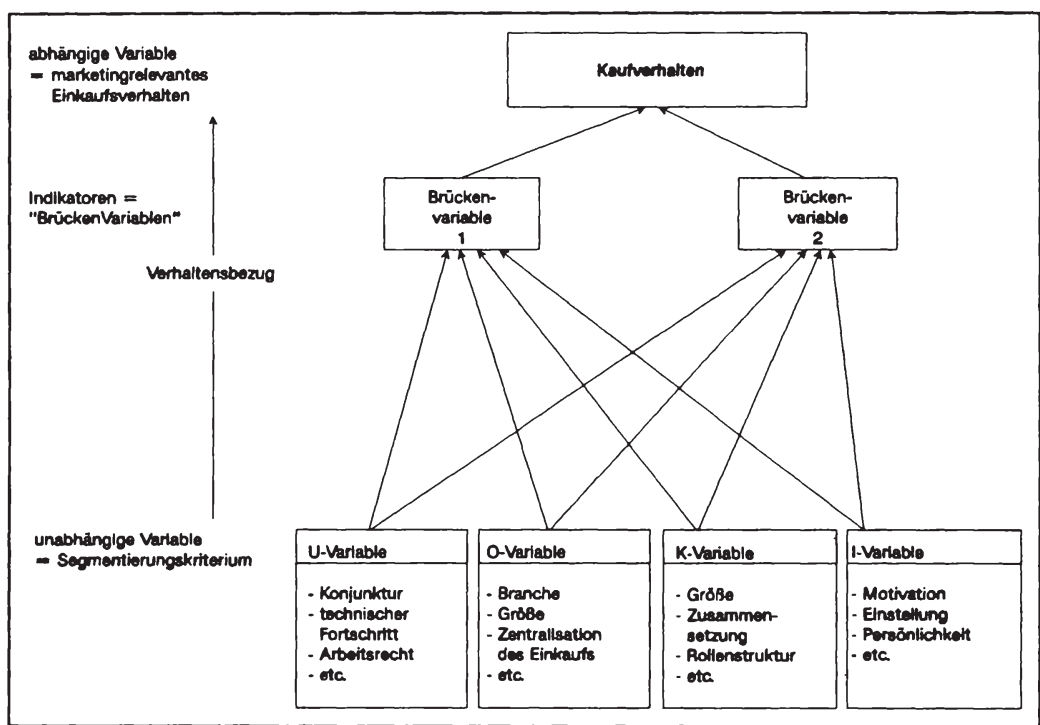

Abbildung 9:

Analyse der Verhaltensrelevanz nach Gröne (in Anlehnung an Gröne, A., Marktsegmentierung bei Investitionsgütern, a.a.O., S. 34)

Rollen- und Machtstrukturen. Die Mehrzahl der untersuchten Determinanten eignet sich jedoch allenfalls in geringem Maße als Segmentierungskriterien im

94 Vgl. Gröne, A., Marktsegmentierung bei Investitionsgütern, a.a.O., S. 30 ff. 
Investitionsgütermarketing. 95 Im Hinblick auf kundendienstbezogene Segmentierungsaufgaben ist eine wesentlich problembezogenere Selektion der in die Analyse eingehenden Variablen erforderlich. Hierbei ist vor allem auf eine stärkere Berücksichtigung bedürfnisgenerierender Faktoren zu achten.

Dagegen ist die Operationalisierung der betrieblichen Kaufwahrscheinlichkeit ein zentraler Fortschritt im Bereich der investitiongüterorientierten Segmentierungsforschung. So liegt Grönes Ansatz, eine Verbindung zwischen objektiv beobachtbaren Variablen und lediglich indirekt erfaßbaren Merkmalen mit Hilfe von Brūckenvariablen herzustellen, bereits weiterührenden Forschungsarbeiten zu Grunde.96

Insgesamt unterliegt dieses Modell der gleichen Kritik wie der mehrstufige Segmentierungsansatz von Wind/Cardozo. Darüber hinaus wird Grönes Modell den Anforderungen einer strategischen Segmentierung nur bedingt gerecht, da es im Rahmen der Marktbearbeitung erhebliche Defizite aufweist. Sein zentraler Erkenntniswert liegt vielmehr in der differenzierten Aufarbeitung des betrieblichen Kaufverhaltens. Der Marktbearbeitungsbezug ist aber gerade für eine strategische Servicesegmentierung von elementarer Bedeutung. Dies führt zu einer begrenzten praktischen Umsetzbarkeit seines Modells. Vor allem aus dem Konzept der Brückenvariablen sind jedoch geeignete Ansatzpunkte für eine Verwendung im Rahmen der Servicesegmentierung abzuleiten.

\subsection{Bewertung bestehender Segmentierungsansätze in bezug auf inve- stive Kundendienstleistungen}

Ihren wesentlichen Beitrag zur Segmentierungsforschung haben die mehrstufigen Ansätze insbesondere durch eine ausführliche Auseinandersetzung mit dem betrieblichen Kaufverhalten geleistet. So hat die explizite und z.T. sehr differenzierte Berūcksichtigung betrieblicher Kaufprozesse und -strukturen zu einer we-

95 Vgl. Gröne, A., Marktsegmentierung bei Investitionsgütern, a.a.O., S. 190; Kols, P., Bedarfsorientierte Marktsegmentierung auf Produktivgütermärkten, a.a.O., S. 75.

96 Vgl. Horst, B., Ein mehrdimensionaler Ansatz zur Segmentierung von Investitionsgütermärkten, a.a.O., S. $135 \mathrm{ff}$. 
sentlich größeren Transparenz in bezug auf das industrielle Entscheidungs- und Kaufverhalten geführt.

Da auch die Betrachtung investiver Serviceleistungen fraglos mit Aspekten eines multipersonalen Kaufverhaltens von Unternehmen verknüpft ist, bietet sich ein mehrstufiges Vorgehen grundsätzlich an. Zusätzlich zu berücksichtigen ist dabei jedoch, daß bei der Kundendienstsegmentierung bereits ein Erkenntnisvorsprung durch das Wissen über Kaufprozesse und grundservicespezifische Entscheidungsstrukturen beim Hauptleistungskauf besteht. Abhängig von den innerbetrieblichen Verflechtungen des Hauptleistungs- und des Kundendienstvertriebs ergeben sich diesbezüglich möglicherweise beachtenswerte Synergiepotentiale für Serviceanbieter.

Einhergehend mit einem komplexeren Verfahrensaufbau sind aber auch die Anforderungen an den Umfang und die Zusammenstellung geeigneter Segmentierungskriterien zur Bildung homogener Cluster gestiegen. Dies schränkt die praktische Umsetzbarkeit der bestehenden Vorschläge erheblich ein. So steht dem beachtlichen Aufwand in bezug auf die Datenbeschaffung und -auswertung u.U. ein nur geringer Erkenntnisgewinn für die Marktbearbeitung gegenüber. Läßt sich der Aspekt der Datenauswertung noch mit dem Verweis auf moderne Hilfsmittel und Instrumente der elektronischen Datenverarbeitung entkräften, stellen jedoch grundlegende Bedenken, wie etwa die von Gröne bezüglich der Eignung seiner Kriterien zur Segmentierung, eine ernstzunehmende Einschrānkung dar. Hinsichtlich kundendienstbezogener Aufgaben muß infolgedessen die Auswahl tatsächlich relevanter Kriterien mit besonderer Sorgfalt erfolgen.

Insgesamt muß die Eignung mehrstufiger Segmentierungsansätze für Probleme des Kundendienstmarketing differenziert beurteilt werden. Kein Ansatz ist unmittelbar übertragbar für die Probleme von investiven Serviceleistungen. Die Diskussion von Stärken und Schwächen der unterschiedlichen Segmentierungsvorschläge hat jedoch zu Implikationen für eine kundendienstorientierte Segmentierung geführt. Zusammengefaßt besitzen folgende Gesichtspunkte zentrale Bedeutung:

- Mehrstufige Betrachtung

Eine Untersuchung investiver Serviceleistungen erfordert zwangsläufig eine Analyse des komplexeren, multipersonalen Kaufverhaltens von Unternehmen. Sie muß demzufolge mehrstufig angelegt sein. Obgleich sich die multi- 
personale Rollen- und Machtsituation bei kundendienstbezogenen Kaufprozessen im Gegensatz zu Produktkäufen verändert darstellt, erscheint der Segmentierungsvorschlag von Wind/Cardozo als geeigneter Ausgangspunkt.

- Indirekte Erfassung servicebezogener Bedürfnisse

Die kundendienstbezogene Bedürfnissituation von Unternehmen als grundlegende Bestimmungsgröße für das jeweilige Inanspruchnahmeverhalten (Kaufverhalten) von Serviceleistungen ist nicht unmittelbar erfaß- und meßbar. In Bezug auf ein zu entwickelndes Segmentierungskonzept für Kundendienstleistungen, ist daher entsprechend den Ausführungen von Gröne eine Verbindung zwischen dem Inanspruchnahmeverhalten von Kundendienstleistungen, bzw. der grundlegenden servicespezifischen Bedürfnislage als abhängige Größe und unabhängigen, erfaßbaren Ausgangsvariablen herzustellen. Erst mit Hilfe derartiger Brükkenkonstrukte wird die grundsätzliche Möglichkeit einer bedürfnisbezogenen Kundendienstsegmentierung geschaffen.

- Messung von Marketingreaktionen

Demographische und psychographische Merkmale bilden bei nahezu allen Segmentierungsaufgaben einen zentralen Bestandteil. Auch im Rahmen der Servicesegmentierung tragen sie in unverzichtbarem Maße zur Beschreibung und Identifikation von Segmenten bei. Um eine bessere Ausrichtung auf das reale Marktgeschehen sicherzustellen, bietet sich die Einbeziehung reaktionsbezogener Größen an. Die Verwendung solcher Kriterien stellt in hohem Maße eine sinnvolle Ergänzung des klassischen Kriterienkataloges dar. Durch die Erfassung unterschiedlicher Reaktionen auf die Marketingimpulse von Anbietern, ergeben sich unmittelbare Ansatzpunkte für die zielgruppengerechte Gestaltung des Marketinginstrumentariums. Damit werden bereits in der Entwicklungsphase der Segmentierungskonzeption die für eine strategische Marktsegmentierung erforderlichen Schwerpunkte auf Aspekte der Marktbearbeitung gelegt. 


\section{Die Operationalisierung des Kundendienstbedarts von Unternehmen}

\subsection{Brückenvariablen als Mittel zur Operationalisierung des Kunden- dienstbedarts}

Grundlegend für die Kundendienstnachfrage sind die bestehenden Servicebedürnisse einzelner Unternehmen. 97 Diese konkretisieren sich in spezifischem Bedarf, der wiederum zu Nachfrage nach Kundendienstleistungen führt. Die verschiedenen Servicebedürfnisse von Unternehmen bilden damit als abhängige Variable den Bezugspunkt einer Kundendienstsegmentierung.

In der Regel wird man jedoch zwischen der servicebezogenen Bedürfnissituation als Outputgröße und den verschiedenen Segmentierungskriterien keine eindeutige und unmittelbare Beziehung herstellen können. Eine direkte "Meßbarkeit" der abhängigen Variablen ist somit nicht möglich.

Daher sollen im folgenden sog. "Brückenvariablen" zur Messung von Kundendienstbedürnnissen gebildet werden. 98 Hierbei handelt es sich um theoretischen Konstrukte, die als "Zwischenglied" in die Beziehung zwischen Kundendienstbedürfnissen und Segmentierungskriterien "eingeschoben" werden (vgl. Abb. 10). 99

Brūckenvariablen bestimmen dabei einerseits in hohem Maße die servicebezogene Bedürfnissituation und sind daher zu einer inhaltichen Segmentabgrenzung in vergleichbarer Weise geeignet wie die Bedürfnisvariable selbst. Andererseits sind sie mit Hilfe verschiedener Segmentierungskriterien meßbar, und damit im Gegensatz zum "Kundendienstbedürfnis" selbst im Rahmen einer empirischen Analyse ursprünglich abbildbar. ${ }^{100}$ Insofern besitzen all diejenigen

97 Zur Problematik des "Bedürfnis"-Begriffs sowie der Messung der Bedürnisbefriedigung vgl. Dichtl, E., Ansatzpunkte zur Messung der Bedürnisbefrledigung, in: Die Unternehmung, 29. Jg., 1975, Nr. 1, S. 1 ff.

98 Vgl. hierzu in Kap. B.1.2 insb. Abb. 9.

99 Vgl. zur Verwendung von Brückenvariablen Gröne, A., Marktsegmentierung bei Investitionsgütern, a.a.O., S. $34 \mathrm{ff}$.

$100 \mathrm{Zu}$ diesem Vorgehen vgl. auch die Ausführungen bei Sheth, der einen ähnlichen Bezugsrahmen zur Segmentierung von Konsumgütermärkten entwickelt. Vgl. Sheth, J.N., Marktsegmentierung als relevante Planungshilfe des Marketing, a.a.O., S. $124 \mathrm{ff}$. 


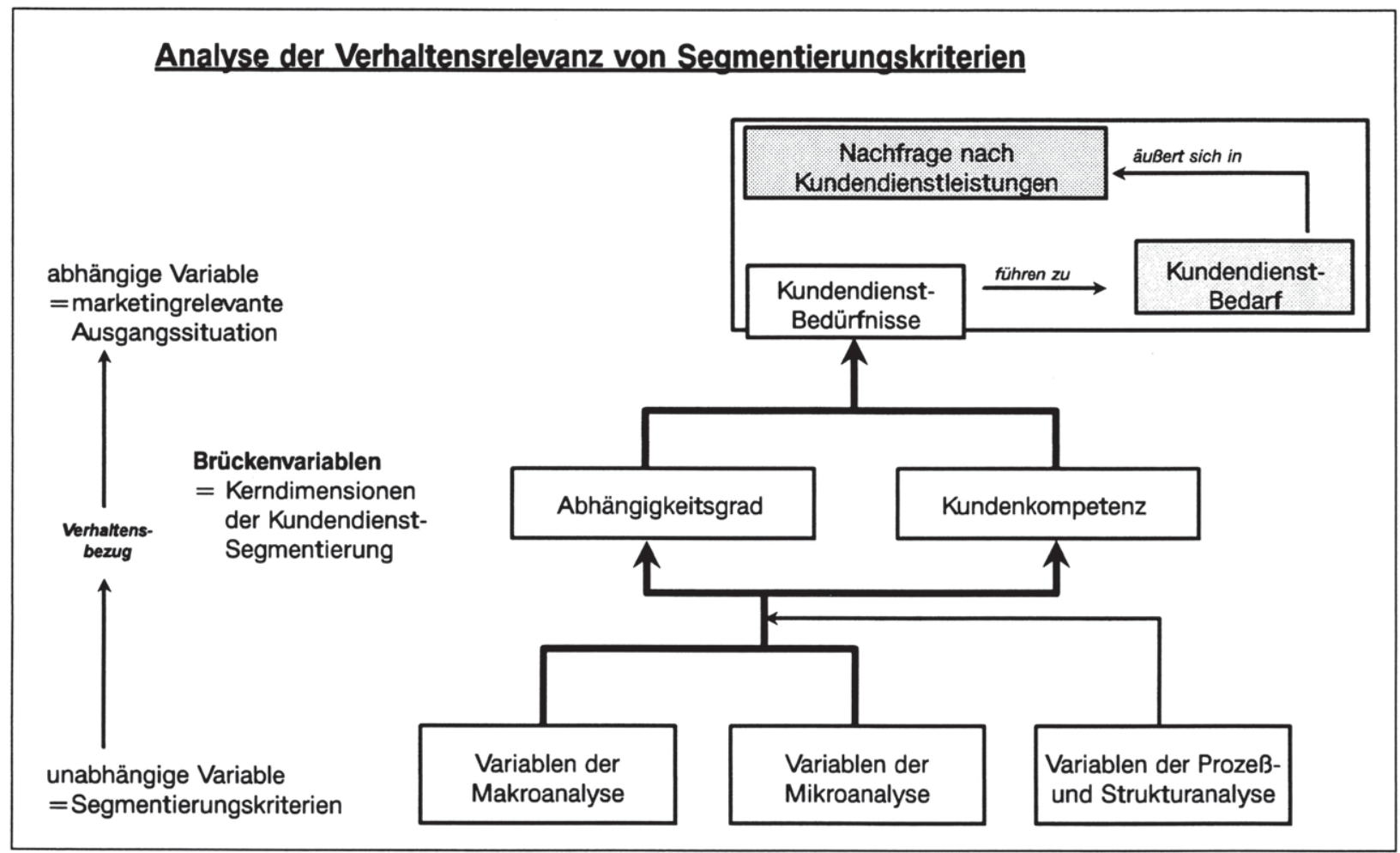

Abbildung 10: Konstrukt der Verhaltensrelevanz von Segmentierungskriterien 
Variablen, die einen Bezug zu einer oder zu beiden Brückenvariablen autweisen, auch einen Bezug zur jeweiligen Ausprägung der Bedürfnissituation.

Im Rahmen der vorliegenden Untersuchung stehen zwei Konstrukte im Mittelpunkt des Betrachtung: Der "Abhängigkeitsgrad" der Unternehmen von ihren PCs (1. Brückenelement) und die "Kompetenz", mit der Personal Computer in den Unternehmen eingesetzt und betrieben werden (2. Brūckenelement). Mit Hilfe dieser beiden Komponenten sollen die kundendienstbezogenen Bedürtnisse von Unternehmen differenziert werden. Deshalb steht die spezielle Bedeutung dieser beiden Konstrukte zunāchst auch im Mittelpunkt der weiteren Ausführungen. Im Rahmen einer ausführlichen Diskussion ist die besondere Relevanz dieser beiden Brūckenvariablen "Abhängigkeitsgrad" und "Kundenkompetenz" für den vorliegenden Zusammenhang zu erläutern. Dies geschieht vor dem Hintergrund des dieser Untersuchung zugrunde gelegten Kundendienstmarktes für kommerzielle Personal Computer. Im Anschluß daran werden die kundendienstbezogenen Ausgangsvariablen vorgestellt.

\subsection{Kundendienstbezogene Brückenvariablen}

\subsection{Der hauptlelstungsbezogene Abhänglgkeltsgrad}

Die erste grundlegende Dimension für Kundendienstbedürfnisse, der Abhängigkeitsgrad, wird vorwiegend durch verwendungsbezogene Aspekte bestimmt. Er ist festgelegt durch die Art, wie PC-Systeme in den jeweiligen Unternehmensprozeß integriert sind. Dahinter steht die Kernfrage, wie wichtig PCs für die Aưgabenverrichtung im Unternehmen, in einzelnen Unternehmensbereichen oder Einzelabteilungen sind.

Der Stellenwert, den ein Arbeitsmittel aufweist, hängt in entscheidendem Maße von dessen Beitrag im Arbeitsprozeß eines Unternehmens ab. Der Faktor "Information", und damit die verbundenen Technologien zur Gewinnung, Behandlung und Verarbeitung von Informationen, gewinnt seit geraumer Zeit zunehmend zentrale Relevanz in allen Wirtschaftsbereichen. Dies betrifft sowohl die Bedeutung der Information als "Produktionsfaktor" bzw. ihren Beitrag in den 
einzelnen Phasen des betrieblichen Wertschöpfungsprozesses. ${ }^{101}$ Aber auch der erhebliche Einfluß des Informationsmanagements auf die Wettbewerbsposition von Unternehmen (Information als "strategischer Wettbewerbsvorteil") trägt zu dieser Situation bei. 102

Das mit dem Informationsstellenwert gleichsam gestiegene Informationsvolumen ist inzwischen nur noch mit Computertechnologie beherrschbar. Zur Lösung dieser Aufgaben stehen verschiedene Systemalternativen zur Verfügung. Dabei bieten sich neben Mainframe-Computern und mittleren Datenverarbeitungssystemen auch Personal Computer bzw. PC-Netze als geeignete Problemlösung an. 103

Die mit einer solchen Systementscheidung einhergehenden Konsequenzen sind jedoch weitreichend. Aufgrund der vielfältigen Verflechtungen eines Informationssystems sind häufig organisationale, personelle, produktionstechnische u.a. Anpassungsprozesse erforderlich. Mit fortschreitender Technologiediffusion im Unternehmen steigt dabei der Abstimungsbedarf mit dem gewählten Computersystem. Dieser Sacheralt verleiht solchen Technologieentscheidungen strategischen Charakter.

Je nach Ausmaß dieser Verflechtungen, ist die Produktivität eines Unternehmens bzw. einzelner Unternehmensbereiche auf die Funktions- und Einsatzbereitschaft seiner PC-Konfiguration angewiesen. ${ }^{104}$ Abhängig von der jeweiligen

101 Vgl. Reuter, E., Wertschöpfung durch Informationstechnologie, in: Informationstechnologie und strategische Führung, Hrsg.: Spremann, K., Zur, E., Wiesbaden 1989, S. 18 f.; Schüler, W., Informationsmanagement: Gegenstand und organisatorische Konsequenzen, In: Informationstechnologie und strategische Führung, Hrsg.: Spremann, K., Zur, E., Wiesbaden 1989, S. 181.

102 Zum Wesen und zur Bedeutung eines Informationsmanagements vgl. Meffert, H., Medien 2000 - Thesen zu den Entwicklungsperspektiven elektronischer Medien im Marketing, in: Strategische Unternehmensführung und Marketing, Hrsg.: Meffert, H., Wiesbaden 1988, S. 185; Porter, M.E., Millar, V.E., Wettbewerbsvorteile durch Information, in: HARVARDmanager, 1986, Nr. 1, S. 149 f.; Nagel, K., Bewertung strategischer Wettbewerbsvorteile durch Informationssysteme, in: Informationstechnologie und strategische Führung, Hrsg.: Spremann, K., Zur, E., Wiesbaden 1989, S. 49.

103 Zur Unterscheidung unterschiedlicher EDV-Systeme vgl. auch Kap. A.3.

104 Untersuchungen haben sich damit befaßt, wie lange Unternehmen aus verschiedenen Branchen ihre Produkttvität nach einem Ausfall ihres EDV-Systems noch aufrecht erhalten können. Hierbei stellte sich heraus, daß $20 \%$ aller befragten Unternehmen effizient nur noch 
Integrationsbreite und tiefe solcher Systeme definiert sich das Bestreben, Funktionsstörungen und Systemausfälle weitestgehend zu vermeiden bzw. auf ein akzeptables Minimum zu reduzieren. Serviceleistungen stellen diesbezüglich ein probates Mittel dar.

Die Integration von PCs in Unternehmensprozesse kann insgesamt recht unterschiedlich ausgeprägt sein. So sind Personal Computer traditionell vielfach als sporadisch genutzte, "entbehrliche" Arbeitsplatz-Hilfsmittel eingesetzt worden, während zentrale EDV-Anwendungen fast ausnahmslos über größere Systeme betrieben wurden. Die schnelle technologische Entwicklung von PCs und leistungsfähigen PC-Netzen hat diese Situation jedoch nachhaltig verändert. ${ }^{105}$ So werden PCs zunehmend breiter, aber auch tiefer in die Problemlösungsprozesse von Unternehmen eingebunden. Abbildung 11 verdeutlicht, daß mit zunehmendem Abhängigkeitsgrad auch die Kundendienstbedürfnisse von Unternehmen steigen.

Zum Teil werden diese Rechner nach wie vor als unabhängige Einzel-Arbeitsplatzsysteme ("Insellösungen") eingesetzt. 106 In diesem Rahmen dienen sie zumeist als taktisches Arbeitsmittel, das vorwiegend bei administrativen Standardaufgaben (Textverarbeitung, Grafik, Tabellenkalkulation) eingesetzt wird. Eine Systemabhängigkeit ist in diesen Fällen nur bedingt gegeben, da bei auftretenden Stör- und Ausfallsituationen vielfach die Aufgabenerfüllung zeitlich gestreckt oder auf Ersatztechnologie zurückgegriffen werden kann. ${ }^{107}$

Stunden und 48\% nur noch Tage weiterarbeiten können. Bei Banken und Versicherungen liegen diese Werte mit 33\% bzw. 50\% sogar noch höher. Vgl. Young, A., Evens, M., Orr, J., Management Awareness of Computer Risks, 0.0.,1987, S. 12. Zur Computersicherheit vgl. auch Zayac, B.P., Summers, R.C., Computers and Security, 0.O., 1988.

Neueste Untersuchungen des amerikanischen Marktforschungsinstituts Dataquest bestätigen den Trend, daß Personal Computer zunehmend die traditionellen Großrechner-Systeme in der Datenverarbeitung verdrāngen. Vgl. o.V., Rückgang bei Großrechnern - Verlierer IBM, in: Chip, 1993, Nr. 3, S. 26 f.

106 Zum Begriff und zum Wesen von Insellösungen vgl. Spremann, K., Reichling, P., Schwachstellen und Konstruktionsprinzipien beim Systemdesign, Wiesbaden 1989, S. $189 \mathrm{ff}$.

107 Tritt bei einem als Textverarbeitungssytem genutzten PC eine Störung aưf, kann häufig aưf eine Schreibmaschine zurückgegriffen werden. Damit können nahezu alle Aufgaben, ohne zeitliche Verzögerungen durchgeführt werden. 


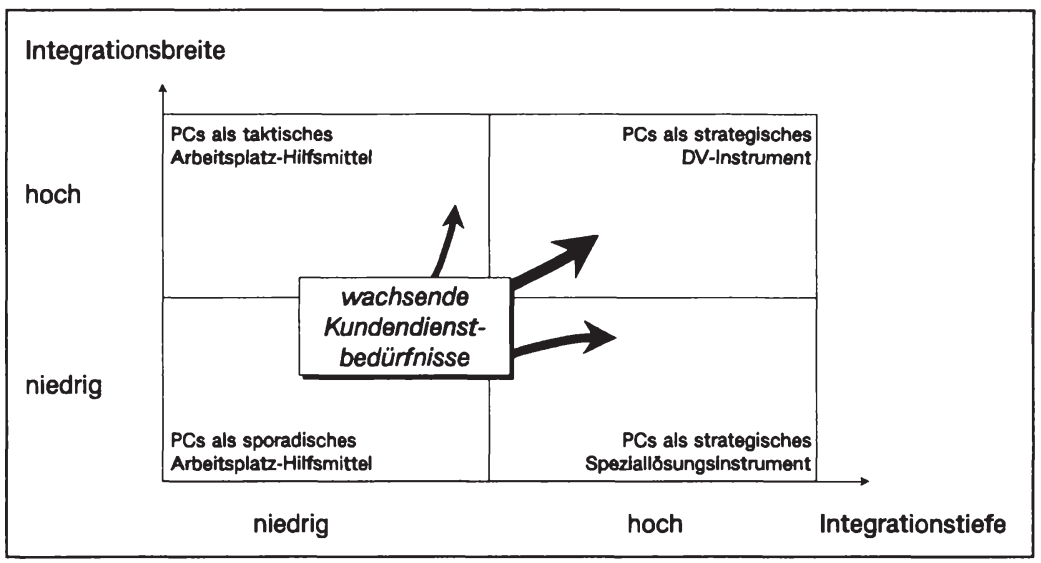

Abbildung 11: Integrationsformen von Personal Computern

Neben dieser Verwendungsrichtung bestehen jedoch auch bereits wesentlich tiefere und damit ausfallsensiblere Einbindungsformen. Dies betrifft zum einen strategische Speziallösungen. Die Aufgabenerfüllung in einzelnen Unternehmensbereichen ist dabei in hohem Maße auf PCs abgestimmt. 108 Obwohl derartige Lösungen i.d.R. eine Ergänzung zu den auf größeren Computern betriebenen Hauptanwendungen der Datenverarbeitung darstellen, sind sie für die Leistungserbringung in ihren Teilbereichen häufig unverzichtbar wichtig. Zum anderen gibt es Unternehmen, die bereits ausschließlich sämtliche DV-Aufgaben mit PC(-Netzen) durchführen und ganz auf zusätzliche Computersysteme verzichten.

Entsprechend den gekennzeichneten Integrationsformen definiert sich auch die kundendienstspezifische Bedürfnislage von Unternehmen in bezug auf ihre PCSysteme. Mit steigender Abhängigkeit ist bei entstehenden Funktionsstörungen die Produktivität des Unternehmens beeinträchtigt. Hieraus entsteht ein Bedarf nach einer geeigneten Absicherung. Im Ergebnis kann damit der Abhängigkeitsgrad als ein erster, wesentlicher Indikator für die kundendienstspezifische Bedürfnissituation von Unternehmen herangezogen werden.

108 So führt z.B. bei PC-gesteuerten Fertigungsstraßen ein Systemausfall fast immer zur Produktionsunterbrechung. Dle Abhängigkeit von solchen PC-Systemen ist ausgesprochen hoch. 
Im einzelnen bestimmt sich die Bedürfnisintensität dabei vor allem durch die Folgen eines möglichen Systemausfalls. In diesem Zusammenhang von Bedeutung sind daher u.a.:

- Möglichkeiten einer Aufgabenerfüllung ohne PCs,

- Ausmaß bzw. Umfang der Aufgabenunterbrechung,

- Kosten und Folgekosten eines Produktivitätsabfalls,

- bestehende Möglichkeiten, auf Ersatztechnologien zurückgreifen zu können.

\subsection{Die hauptleistungsbezogene Kundenkompetenz}

Die zweite Brückendimension zur Operationalisierung von Kundendienstbedürfnissen ist die Eigenkompetenz der Servicenachfrager (Kundenkompetenz). Sie ist in erster Linie ein verhaltensorientiertes Konstrukt und drückt aus, welches Know-how bzw. welches Wissen im Unternehmen in bezug auf PC-Technologie besteht, d.h. mit welcher Kompetenz der Kunde (= das Unternehmen) seine PCSysteme einsetzt und betreibt. Dahinter steht auch die Frage, wie groß die Möglichkeiten zur Selbsthilfe bei auftretenden PC-Störungen sind. So kann unterstellt werden, daß Unternehmen mit hoher Kompetenz eine geringere kundendienstbezogene Bedürfnislage aufweisen als kompetenzschwache.

Ursprūnglich setzten sich im Rahmen der Betriebswirtschaftslehre besonders die Organisationslehre und das Personalwesen mit Kompetenzaspekten auseinander. 109 Dabei stehen Fragen der Delegation von Kompetenzen, im Sinne der Übertragbarkeit von Rechten und Befugnissen an nachgeordnete Stellen in einer Organisationshierachie im Mittelpunkt der Betrachtungen. 110

109 Insgesamt vollzieht sich die wissenschaftliche Auseinandersetzung mit Fragen der Kompetenz interdisziplinär. Neben der Betriebswirtschaftslehre haben sich eine Reihe weiterer Forschungsbereiche, insbesondere auch die Psychologie, mit diesem Thema befaBt. Dabei weisen die verschiedenen Untersuchungen z.T. ein recht unterschiedliches "Kompetenz"Verständnis auf.

110 Vgl. z.B. Bleicher, K., Kompetenz, in: Grochla, E., (Hrsg.): Handwörterbuch der Organisation, 2. Aufl., Stuttgart 1980, Sp. 1056 - 1078; Bühner, R., Betriebswirtschaftliche Organisatl- 
Das Marketing hat diese Diskussion aufgegriffen und dabei neue Impulse gesetzt. In diesem Zusammenhang erfolgt jedoch eine stärkere Orientierung an Aspekten des Leistungsvermögens, d.h. Fähigkeiten und Fertigkeiten von Personen und Organisationen. ${ }^{111}$ Dabei steht das spezifische Leistungsvermögen eines Unternehmens im Mittelpunkt. Dies sind charakteristische Kompetenzen ("distinctive competences"), die zur Erlangung von strategischen Wettbewerbsvorteilen von zentraler Bedeutung sind. 112 Mit Hilfe solch eigener Fertigkeiten und Fähigkeiten muß ein Unternehmen bemüht sein, ein eigenständiges und unterscheidbares Leistungsprofil zu entwickeln, um so eine erfolgversprechende Wettbewerbsposition aufbauen zu können. Im Ergebnis handelt es sich hierbei also in erster Linie um eine anbieterbezogene Kompetenzbetrachtung. ${ }^{113}$

Wesentliche Erkenntnisse zur Kompetenzforschung kommen auch aus der Psychologie. Hier wird das jeweilige Leistungsvermögen eines Menschen als ein spezifisches Element seiner Persönlichkeit betrachtet. Die "Kompetenz" repräsentiert eine wesentliche Persönlichkeitsdimension und stellt demzufolge ein wichtiges Unterscheidungsmerkmal gegenüber anderen Individuen dar. ${ }^{114}$ Überträgt man diesen Zusammenhang auf die Situation von Unternehmen, so läßt sich der Schluß ziehen, daß "distinctive competences" eine gleichartige Bedeutung aufweisen wie individuelle "Kompetenzen". Demzufolge sind beide Konstrukte vergleichbar und können als charakteristisches Unterscheidungsmerkmal sowohl für Menschen als auch für Unternehmen herangezogen werden. 115

onslehre, 3. Auff., München 1987, S. 73; Wöhe, G., Einführung in die allgemeine Betriebswirtschaftslehre, 14. Auff., München 1981, S. 160.

111 Zu marketingspezifischen Untersuchungen vgl. u.a. Backhaus, K., Weiss, P.A., Kompetenz: Neue Dimension beim Marketing von Systemtechnologien, in: HARVARDmanager, 1989, $\mathrm{Nr}$. 3, S. 107 ff.; Weiss, P.A., Die Kompetenz von Systemanbietern, Berlin 1992, S. 63 ff. Zu psychologieorientierten Arbelten vgl. besonders Stäudel, Th., Problemlösen, Emotionen und Kompetenz, Regensburg 1987, S. $47 \mathrm{ff}$. und die dort angegebene Literatur.

112 Zum Begriff der "distinctive competences" vgl. Hofer, Ch.W., Schendel, D., Strategy Formulation, St. Paul, Minnesota 1978, S. 25.

113 Zusätzlich zu dieser Außenwirkung bilden die Eigenschaften gleichzeitig auch den Maßstab für die Qualität der zu produzierenden Leistungen und legen damit das unternehmenseigene Anspruchsniveau fest. Vgl. Lux, P.G.C., Zur Durchführung von Corporate Identity Programmen, in: Birkigt, K., Stadler, M.M, Funcke, H.J., (Hrsg.), Corporate Identity: Grundlagen, Funktionen, Fallbeispiele, 4. Auff., Landsberg am Lech 1988, S. 577.

114 Vgl. Guilford, J.P., Persönlichkeit, Weinheim 1954, S. 6 ff.

115 Vgl. hierzu auch Weiss, P.A., Die Kompetenz von Systemanbietern, a.a.O., S. 60. 
Die Psychologie hat zur Kompetenzforschung besonders auch dadurch beigetragen, daß sie das Kompetenzkonstrukt weiter differenziert hat. ${ }^{116}$ Danach setzt sich die "aktuelle Kompetenz" eines Individuums aus einer epistemischen und einer heuristischen Kompetenzkomponente zusammen. ${ }^{117}$

Die epistemische Kompetenz wird auch als Kompetenz des Fachmanns beschrieben. ${ }^{118}$ Ein solches Wissen wird vor allem durch die ausbildungsbezogenen Inhalte und Erfahrungen aufgebaut. Derartig erworbene Fertigkeiten zur Problemlösungen können zum Kompetenztransfer für vergleichbare Aufgabenstellungen herangezogen werden. ${ }^{119}$

Die heuristische Kompetenz umfaßt das Ausmaß an Fähigkeiten, das sich im Laufe der Zeit durch die Lösung verschiedener bzw. neuartiger Aufgabenstellungen entwickelt hat. Sie spiegelt damit die durch Erfahrung gewonnenen Möglichkeiten eines Individuums zur Problemlösung wieder.

Es bestehen bislang jedoch keine präzisen Aussagen über die unmittelbaren Wirkungszusammenhänge dieser Dimensionen. So weist Stäudel zwar auf einen gewissen Substitutionsspielraum beider Größen hin. Stichhaltige Aussagen zur Operationalisierung und Verknūpfung der Kompetenzkomponenten führt jedoch auch sie nicht an. 120

Besonders vor dem Hintergrund einer empirischen Untersuchung ist zu klären, wie die individuellen Kompetenzzusammenhänge ${ }^{121}$ auf die Situation von Unternehmen übertragen werden können, und wie diese dann zu operationalisieren

116 Vgl. Weis, P.A., Die Kompetenz von Systemanbietern, a.a.O., S. 60 f.; Dörner, D., Intention Memory and Intention Regulation, in: Kix, F., Hagedorn, H., (Hrsg.), Human Memory and Cognittve Capability, North-Holland, Elsevier 1986, S. 929 ff.; Stäudel, Th., Problemlösen, Emotionen und Kompetenz, a.a.O., S. 54 ff.; Stäudel, Th., Der Kompetenzfragebogen, in: Diagnostica, Nr. 34, 1988, S. $136 \mathrm{ff}$.

117 Stăudel, Th., Problemlösen, Emotionen und Kompetenz, a.a.O., S. 47.

118 Vgl. Weiss, P.A., Die Kompetenz von Systemanbietern, a.a.O., S. 61.

119 Vgl Backhaus, K., Investitionsgütermarketing, a.a.O., S. 368.

120 Vgl. Stäudel, Th., Problemlösen, Emotionen und Kompetenz, a.a.O., S. 55.

121 Im weiteren Verlauf dieser Untersuchung wird von einer Kompetenz nur noch im Sinne bestehender Fähigkeiten und Fertigkeiten von Unternehmen im Umgang mit PCs gesprochen, also einer Eigenkompetenz von PC-Kunden. 
sind. Dies betrifft sowohl die Auswahl der zu betrachtenden Variablen als auch die Kombination und Verknüpfung ihrer Wirkungseffekte.

Zur inhaltlichen Erfassung der Kundenkompetenz bieten sich zunächst grundsätzlich zwei alternative Vorgehensweisen an. Zum einen kann versucht werden, dieses Konstrukt durch geeignete Kompetenzindikatoren zu erfassen (objektive Kompetenzbestimmung). Dies sind Argumente, die fachbezogenes Wissen fördern bzw. prägen. Entsprechende kompetenzbeeinflussende Indikatoren sind z.B.:

- das PC-bezogene Informationsverhalten (Menge und Häufigkeit)

- der Ausbildungsstand betroffener Personen (EDV-spezifische oder -fremde Berufsausbildung)

- das Weiterbildungsverhalten in bezug auf PC-bezogene Themen (Schulung, Seminare, Tagungen)

- Erfahrungen mit PC-vergleichbaren Systemen (z.B. Großrechnererfahrungen)

Zum anderen bietet sich die Möglichkeit an, Kompetenz durch Selbsteinschätzung zu erfassen (subjektive Kompetenzbestimmung). Hierbei stuft sich der Betroffene anhand einer Ratingskala bezüglich kompetenzcharakterisierender Statements selbst ein. 122

Die Kompetenzmessung mit Hilfe von Indikatoren erscheint zunächst präziser und frei von subjektiven (Fehl-)Gewichtungen zu sein. Zudem lassen sich die hinzugezogenen Indikatoren relativ eindeutig epistemischen und heuristischen Einflußkategorien zuordnen. Als gravierender Nachteil erweist sich jedoch die Unkenntnis über das Zusammenwirken dieser Einflüsse. Gerade in Anbetracht bestehender Interdependenzen und Substitutionseffekte in bezug auf das Zusammenspiel einzelner Bestandteile können keinerlei gesicherte Aussagen über

122 Stäudel unterstreicht diese Vorgehensweise indem sie feststellt, daß die "... aktuelle Kompetenz ... eine subjektive Einschätzung der eigenen Fähigkeiten, die man für die Bewältigung eines vorliegenden Problems zu haben glaubt, dar(stellt).", Stäudel, Th., Problemlösen, Emotionen und Kompetenz, a.a.O., S. 54. 
deren unabhängige Wirkung getroffen werden. ${ }^{123}$ Aus diesen Gründen wird eine solche Vorgehensweise nicht weiter verfolgt.

Eine Kompetenzerfassung und -messung über subjektive Eigenurteile erscheint dagegen tragfähig und praktikabel. Mit Hilfe einer Reihe verschiedener kompetenzcharakterisierender Aspekte (z.B. kennt sich mit PCs gut aus, arbeitet regelmäßig mit PCs, entwickelt eigene Anwendungen u.a.) läßt sich ein entsprechende Know how von Personen einschätzen. 124

Von zentraler Bedeutung ist hierbei auch, daß gerade die subjektive Einschätzung der eigenen Fähigkeiten und Fertigkeiten ausschlaggebend ist für die Festlegung der Bedürfnissituation. So ist beispielsweise durchaus denkbar, daß ein EDV-Beauftragter zwar objektiv gesehen über bestimmte Servicekenntnisse verfügt, jedoch kein Zutrauen besitzt bzw. die Verantwortung für derartige Arbeiten nicht tragen möchte und somit einen externen Kundendienstleister beauftragt. Trotz objektiv vorhandener Kompetenzen determiniert damit die subjektive Beurteilung die servicespezifische Bedürfnissituation.

Über eine individuelle Betrachtungsebene psychologieorientierter Forschungsarbeiten hinaus, gilt es im vorliegenden Fall jedoch eine unternehmensweite, d.h. multipersonelle PC-bezogene Kompetenz abzubilden. Zum Problem erhebt sich dabei insbesondere die Auswahl eines geeigneten Aggregationsverfahrens.

Die unterschiedlichen Möglichkeiten des strukturellen Erscheinungsbildes einer unternehmensweiten PC-Kompetenz verschärfen diese Problematik zusätzlich. Hierbei ist außer den jeweiligen Kompetenzständen einzelner Mitarbeiter oder Funktionsgruppen vor allem auch die im Unternehmen vorhandene Kompetenz-

123 Zwar reichen weder epistemische noch heuristische Kompetenzbestandteile alleine zu einer erfolgreichen Bewältigung von Problemstellungen aus. In gewissem Umfang kann jedoch mangelndes Faktenwissen durch ein hohes $\mathrm{MaB}$ an heuristischer Kompetenz ausgeglichen werden. Vgl. Weiss, P.A., Die Kompetenz von Systemanbietern, a.a.O., S. 61; Stäudel, Th., Emotion, Kompetenz - Vertrauen in sich selbst, in: Absatzwirtschaft, Sondernummer Oktober 1988, S. 148.

124 Die in diesem Zusammenhang benutzte Statementbatterie wurde aus Vorabinterviews mit (kaufmännischen und technischen) PC-Experten großer Computeranbieter entwickelt. Die im Rahmen der Hauptuntersuchung verwendeten Kriterien spiegeln dabei die am hăufigsten genannten Gesichtspunkten wider. Aus diesem Grund sind die venwendeten Statements als hinreichen valide anzusehen. Zu einer ausführlichen Übersicht der verwendeten Kompetenzaspekte vgl. Kap. C.2.2, insb. Abb. 18. 
verteilung zu berücksichtigen. Neben spezialistengeprägten und eher ausgewogenen Kompetenzstrukturen (siehe Struktur $c$ und $a$ in Abb. 12) lassen sich diesbezüglich vielfältig weitere Mischformen, z.B. basisbestimmte oder dezentral geprägte Kompetenzgefüge ( $b$ und $d$ ) anführen.

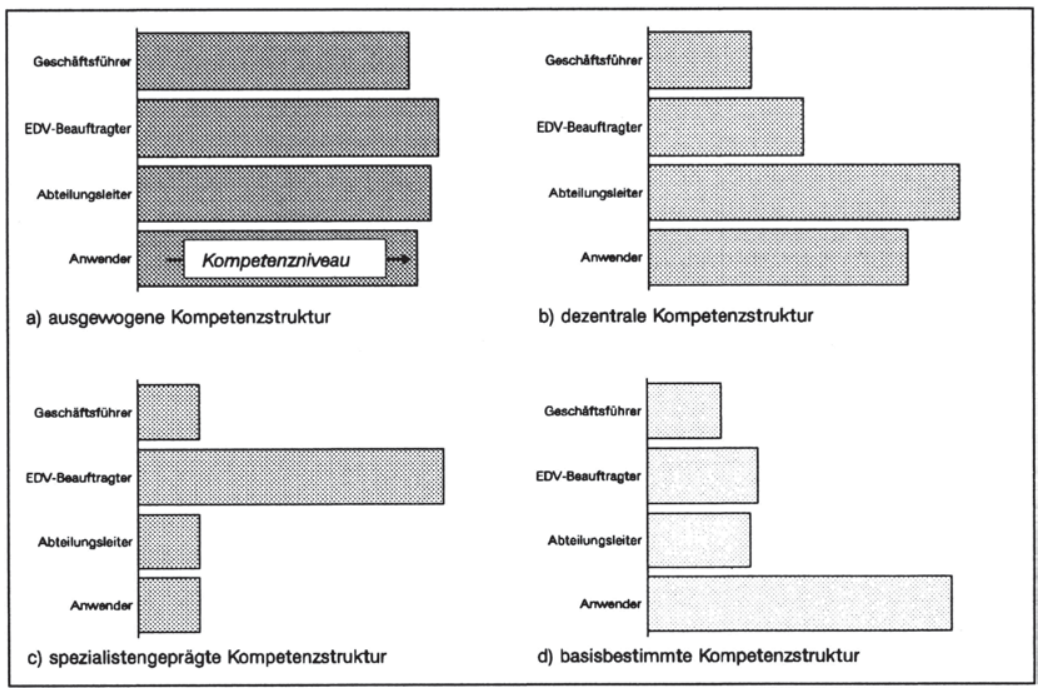

Abbildung 12: PC-bezogene Kompetenzstrukturen 125

Eine Lösung dieser vielschichtigen Problematik setzt ein komplexes Aggregationsmodell voraus, dessen Verknüpfungsregeln bzw. -algorithmen völlig neu hergeleitet werden müssten. Diese Aufgabenstellung ist im Rahmen dieser Arbeit nicht lösbar. Statt dessen bietet sich jedoch eine andere Vorgehensweise an.

Alternativ zu einer aggregierten Kompetenzmessung kann im Unternehmen bereits vorhandenes Know how genutzt werden. Hierbei bietet sich eine stellvertretende Kompetenzbewertung aller beteiligten Unternehmensmitglieder bzw. gruppen durch den EDV-Beauftragten als sinnvolle Lösung an. Aufgrund seiner spezifischen Verantwortlichkeit für DV-Aufgaben ist inm a priori grundlegendes

125 Aus Gründen der Übersichtlichkelt werden die verschledenen Kompetenzstrukturen in Abbildung 11 exemplarisch anhand ausgewählter Hierarchlegruppen (Geschäftsführer, EDVBeauftragter, Abteilungsleiter, Anwender) dargestellt. 
Know how zuzusprechen. Zudem gewährleistet inm seine fachliche Zuständigkeit darüber hinaus einen detaillierten Überblick über das EDV-bezogene Leistungsvermögen der Mitarbeiter im Unternehmen.

Diese Vorgehensweise gewährleistet eine konsistente und damit tragfähige Erfassung PC-bezogener Unternehmenskompetenzen. Die Operationalisierung im Sinne einer weiterführenden Datenverdichtung auf zentrale, unabhängige Kompetenzkomponenten kann schließlich im Rahmen der empirischen Analyse mit Hilfe geeigneter statistischer Methoden erfolgen. ${ }^{126}$

Insgesamt stellt damit das Kompetenzkonstrukt ebenso wie der Abhãngigkeitsgrad eine systematisch hergeleitete und nachvollziehbare Bestimmungsgröße der kundendienstbezogenen Bedürfnissituation von Unternehmen dar. Beide bilden zusammen die Grundlage zur unternehmensbezogenen Kundendienstsegmentierung.

\subsection{Kundendienstbezogene Ausgangsvariablen}

\subsection{Variablen der Makrosegmentlerung}

Die Variablen der Makrosegmentierung umfassen Kriterien, die sich auf einer übergeordneten Ebene mit Aspekten des Unternehmens befassen. Merkmale einzelner Personen bleiben damit auf dieser Stufe noch weitestgehend unberũcksichtigt. Im einzelnen werden vier Kriteriengruppen unterscheiden:

(1) unternehmensbezogene Kriterien

(2) verwendungsbezogene Kriterien

(3) hauptleistungsbezogene Kriterien

(4) organisatorische Institutionalisierungskriterien der EDV-Verantwortlichkeit

126 In der vorliegenden Untersuchung wurde hierzu eine explorative Faktorenanalyse durchgeführt. Zu methodischen Aspekten sowie zur Verdichtung der Kundenkompetenzvariablen selbst vgl. Kapltel C.3.11. 
(1) Mit Hilfe unternehmensbezogener Kriterien werden zunächst generelle Kennzeichen des jeweils untersuchten Unternehmens erfaßt. Hierbei handelt es sich vorwiegend um unternehmensdemographische und -psychographische Merkmale. Im Rahmen einer PC-bezogenen Kundendienstsegmentierung besitzen besonders auch solche Gesichtspunkte Relevanz, die die EDV-, PC- und kundendienstbezogene Situation des Unternehmens kennzeichnen.

Unternehmensbezogene Kennzeichen sind verhältnismäßig leicht und kostengünstig zu gewinnen sowie unproblematisch zu messen. Ihre zeitliche Stabilität ist relativ hoch. Ein Einfluß auf die kundendienstspezifische Bedürfnislage ist vor allem durch kompetenzbestimmende Aspekte gegeben, womit die Bedeutung dieser Kriterien unterstrichen wird. Der Marktbearbeitungsbezug dieser Variablen ist dagegen eher gering zu beurteilen. Implikationen für die Auswahl und Gestaltung einzelner Marketinginstrumente ergeben sich kaum.

(2) Zu der zweiten Gruppe makroanalytischer Kriterien gehören Merkmale, die das Verwendungsverhalten von PC-Systemen im Unternehmen beschreiben. Dies betrifft im einzelnen:

- Verwendungsort

- Verwendungsrichtung

- Verwendungsart

- Verwendungsintensität

- Verwendungsbedeutung (wo?), (wofür?), (wie?), (wie stark?) und (warum?).

Die zeitliche Stabilität dieser Kriterien ist differenziert zu betrachten. Zwar hat die generelle Entscheidung, bestimmte Technologien in Unternehmensabläufe einzugliedern, strategischen Charakter. Ausmaß und Intensität dieser Technologieintegration können sich im Zeitablauf jedoch u.U. ändern. Bedenken hinsichtlich der Meßbarkeit ergeben sich dagegen kaum.

In bezug auf die kundendienstbezogene Bedürfnissituation kann den Kriterien vor allem aufgrund der abhängigkeitsgradbezogenen Gesichtspunkte ein wichtiger Einfluß zuerkannt werden. Auch ein Marktbearbeitungsbezug ist festzustellen. Dies betrifft besonders produktpolitische Fragen. So las- 
sen sich z.B. aus der Integrationstiefe der PC-Systeme Hinweise für die Gestaltung von Serviceleistungen gewinnen (z.B. Reaktionszeiten).

(3) Eine dritte Gruppe bilden hauptleistungsbezogene Kriterien. Als Bezugsund Verrichtungsobjekt von Kundendienstleistungen besitzt die genaue Kenntnis der jeweiligen PC-Konfiguration in Unternehmen besonderes Gewicht. Die Beschreibung und Klassifizierung erweist sich jedoch aufgrund der beiden Systembestandteile Hardware und Software als besonders problematisch. Beide existieren in einer nur sehr schwer zu fassenden Erscheinungsvielfalt, was letztendlich zu einer großen Anzahl möglicher Konfigurationsvarianten führt. Aus diesem Grund gestaltet sich die Aufnahme PC-bezogener Detailaspekte in der vorliegenden Untersuchung auch verhältnismäßig umfangreich.

Die Meßbarkeit hauptleistungsbezogener Kriterien ist ebenso wie die verhältnismäßig einfache Erfassung positiv zu bewerten. Ihr Bedürfnisbezug ist zudem unmittelbar evident. Dabei ist zu vermuten, daß die kundendienstbezogene Bedürfnissituation mit zunehmender Komplexität der Hauptleistung wächst.

Die zeitliche Stabilität der Kriterien ist zumindest mittelfristig gegeben, da PC-Systeme den Betriebsmitteln eines Unternehmens zuzurechnen sind und als Investitionsgüter zumindest einen längeren Zeitraum im Unternehmen verbleiben. Der Grad ihrer technologischen Veralterung kann als plausibler Indikator für die verbleibende Einsatzzeit herangezogen werden. 127

Ein Marktbearbeitungsbezug ergibt sich vor allem im Hinblick auf Aspekte der Serviceproduktpolitik. Dies betrifft insbesondere technische Anforderungen in bezug auf die Wartung und Reparatur unterschiedlicher Systemund Baugruppen. Ansatzpunkte für die Gestaltung der übrigen Marketinginstrumente lassen sich jedoch aus hauptleistungsbezogenen Merkmalen kaum gewinnen.

127 Zum Konzept des Technologie-Lebenszyklus vgl.: Meffert, H., Marketing, a.a.O., S. 64 f.; Little, A.D., Management der F\&E-Strategie, Wiesbaden 1991, S. 65 ff.; Sahal, D., Patterns of Technological Innovation, London u.a. 1981, S. $205 \mathrm{ff}$. 
(4) Mit einer vierten Gruppe makrobezogener Segmentierungskriterien wird erfaßt, inwieweit eine Zuständigkeit und Verantwortung für das EDV- bzw. PC-Wesen in der Unternehmensorganisation verankert ist. Diese Kriterien besitzen vor allem für den weiterführenden Segmentierungsprozeß große Bedeutung. Die Identifikation der in Unternehmen für Kundendienstfragen kaufverhaltensrelevanten Personen kann hierdurch erheblich vereinfacht werden.

Die Institutionalisierung kann dabei entweder durch die Bestellung eines EDV-Beauftragten, oder durch Einrichtung einer eigenständigen EDV-Abteilung geschehen. Einen Hinweis auf eine spezielle Verantwortlichkeit gibt auch die Existenz eines eigenständigen EDV-Budgets.

Die Erfaßbarkeit dieser Kriterien ist unproblematisch. Auch ihre zeitliche Beständigkeit ist gegeben, da organisatorischen Aspekten ein strategischer Charakter beigemessen werden kann. Die Kriterien können ferner als wichtiger Filter für die Ermittlung von weiteren kaufverhaltensrelevanten Aspekten angesehen werden. Ebenso offenbaren sie erste Implikationen für instrumentale Fragen. So hat die Identifikation von Zielpersonen gerade für die Kommunikationspolitik besondere Bedeutung.

\subsection{Variablen der Prozeß- und Strukturanalyse}

Im Rahmen der Prozeß- und Strukturanalyse werden die Rollen- und Machtstrukturen betrieblicher Kaufentscheidungsprozesse aufgedeckt. Diese Strukturen werden analog auf die Kaufsituation von PC-Systemen und Kundendienstleistungen übertragen.

Vorrangiges Anliegen ist es hierbei einerseits, die Personen zu identifizieren, die servicebezogene Kaufentscheidungen maßgeblich prägen und bestimmen. Diese stellen dann im Rahmen der Marktbearbeitung relevante Zielpersonen dar. Andererseits soll festgestellt werden, inwieweit sich die Entscheidungsmuster der beiden Kaufsituationen gleichen bzw. voneinander abweichen. Hierbei lassen sich weitere Hinweise für eine differenzierte Marktbearbeitung gewinnen.

Einen Schwerpunkt bildet in diesem Zusammenhang die Erfas-sung der zentralen Kennzeichen des Buying Centers. Neben gruppendemographischen Merk- 
malen (Größe, Zusammensetzung) ist dabei zu untersuchen, welche Personen in Unternehmen letztendlich bestimmte Entscheidungen treffen. Darüber hin-aus sind die Personen zu identifizieren, die maßgeblich die Meinungsbildung dieser Entscheidungsträger beeinflussen. Ferner ist festzustellen, von welchen Gruppen im Unter-nehmen die häufigsten Kaufanregungen kommen.

Die Messung dieser Größen ist problematisch, da Einschät-zungen zur Rollenund Machtsituation i.d.R. stark sub-jektiv geprägt sind. ${ }^{128}$ Zudem ist die Beschaffung von Informationen über die exakte Zusammensetzung des Buying Centers verhältnismäßig aufwendig. Eine akzeptable Lösungs-möglichkeit stellt hierbei jedoch eine Vorauswahl zentraler Funktionsträger dar. Im Rahmen der vorliegenden Untersu-chung wurde der betrachtete Personenkreis eingegrenzt auf Mitglieder der Geschäftsleitung, Abteilungs- und Bereichs-leiter, Anwender und den EDV-Beauftragten.

Ein Bezug zur kundendienstspezifischen Bedürfnissituation sowie Ansatzpunkte zur Gestaltung von Marketinginstrumenten sind kaum gegeben. Hierfür sind vielmehr Variablen der Mikrosegmentierung heranzuziehen. Jedoch geben die Krite-rien der Prozeß- und Strukturanalyse Aufschluß darüber, mit welchen Personen eine solche Individualanalyse durchzufüh-ren ist. 129

Die zeitliche Stabilität der Kriterien ist in hohem Maße an die Beständigkeit der Buying Center-Zusammensetzung gebunden. Diese kann jedoch, was die Einbindungen bestimmter Funktionen in einzelne Entscheidungsprozesse anbelangt (EDV-Verantwortlichkeit), als relativ stabil angesehen werden. ${ }^{130}$

128 Werden beispielsweise Anwender zu ihrem Einfluß aut kundendienstbezogene Entscheidungen befragt, mag es vorkommen, daß sie ihren diesbezüglichen Stellenwert erheblich höher empfinden, als dies ein Mitglied der Geschäftsleitung einschätzt. Vgl. zu diesem Problem der Eigen- und Fremdeinschätzung auch Gröne, A., Marktsegmentierung bei Investitionsgütern. a.a.O., S.102 ff. und 116.

129 Vgl. Wind, Y., Cardozo, R., Industrial Market Segmentation, a.a.O., S. $156 \mathrm{f}$.

130 So wird der EDV-Beauttragte in PC-bezogenen Entscheidungsprozessen sicherlich immer ein Bestandteil des Buying Centers sein. Dabei kann sich jedoch die Person, die diese Position besetzt, im Zeitablauf ändern. 


\subsection{Variablen der Mikrosegmentierung}

Die dritte Variablengruppe zur Kundendienstsegmentierung setzt sich aus mikroanalytischen Kriterien zusammen. Diese beziehen sich auf individuelle Merkmale von Personen, die in Unternehmen das kundendienstspezifische Kaufverhalten maßgeblich bestimmen.

Einen zentralen Stellenwert nimmt hier der EDV-Beauftragte des Unternehmens ein. Aufgrund seiner besonderen Rolle dominiert er maßgeblich die produkt-, kommunikations-, distributions- und kontrahierungsbezogenen Anforderungen und Bedürfnisse seines Unternehmens im EDV-Bereich. Demzufolge gilt es neben klassischen Segmentierungsmerkmalen vor allem Kriterien abzuleiten, mit denen die Akzeptanz verschiedenartiger Marketingstimuli ermittelt werden kann. Als Ordnungsprinzipien bieten sich dabei die vier Bereiche des Marketingmix an.

Meßbarkeitsbedenken in bezug auf die vorgestellten Kriterien ergeben sich kaum. Die meisten dieser Merkmale lassen sich mit Hilfe von Ratingskalen erfassen und sind somit relativ einfach zu messen. Die zeitliche Stabilität ist differenziert zu beurteilen. So repräsentieren die Antworten das gegenwärtig bestehende Meinungsbild des Befragten zu Kundendienst- und PC-bezogenen Themen. Eigene Erfahrungen, marktgegebene und umweltbezogene Einflüsse, aber auch Impulse aus dem eigenen Unternehmen können im Zeitablauf zu Einstellungsänderungen führen. Aufgrund ihres eher rationalen Wesens kann Einstellungen jedoch eine gewisse zeitliche Beständigkeit zugebilligt werden. ${ }^{131}$

Der Einfluß mikroanalytischer Segmentierungskriterien auf die servicebezogene Bedürnissituation von Unternehmen ist sehr hoch. Dies betriftt besonders die Kompetenzdimension. Gerade die Person des EDV-Beauftragen ist diesbezüglich besonders prägend.

Die besondere Bedeutung der mikroanalytischen Kriterien liegt in ihrer hohen Aussagekraft bezüglich der Gestaltung einzelner Marketinginstrumente. Damit wird die besondere Eignung dieser Kriterien, gerade im Rahmen der strategischen Marktsegmentierung unterstrichen.

131 Einstellungen sind innere Bereitschaften auf bestimmte Umweltimpulse konsistent zu reagieren. Sie beinhalten gleichzeitig immer auch eine Gegenstandsbeurteilung. Vgl. Meffert, $H_{\text {., }}$ Marketingforschung und Käuferverhalten, a.a.O., S. 55. 


\section{Kundendienstpolitische Marktsegmentierung als zweistufiges Problem}

Aufbauend auf der Diskussion über die Bewertung der Relevanz einzelner Kriterien für die Kundendienstsegmentierung gilt es nun, den Ablauf des Segmentierungsprozesses zu erarbeiten. Den Ausgangspunkt der Überlegungen bildet das zweistufige Segmentierungsmodell von Wind/Cardozo mit seinen Ebenen der Makroanalyse sowie der Prozeß- und Strukturanalyse und der Mikroanalyse. 132

Wird das Konzept auf seine drei wesentlichen Kernelemente reduziert, so wird deutlich, daß der Prozeß- und Strukturanalyse in bezug auf den verfahrenstechnischen Ablauf des Modells eine zentrale Bedeutung zukommt. Durch die Analyse der Rollensituation bei betrieblichen Kaufentscheidungen wird die Relevanz verschiedener Personen für solche Prozesse aufgedeckt und bewertet. Die Prozeß- und Strukturanalyse vereinigt damit sowohl segmentierungsbezogene Auf

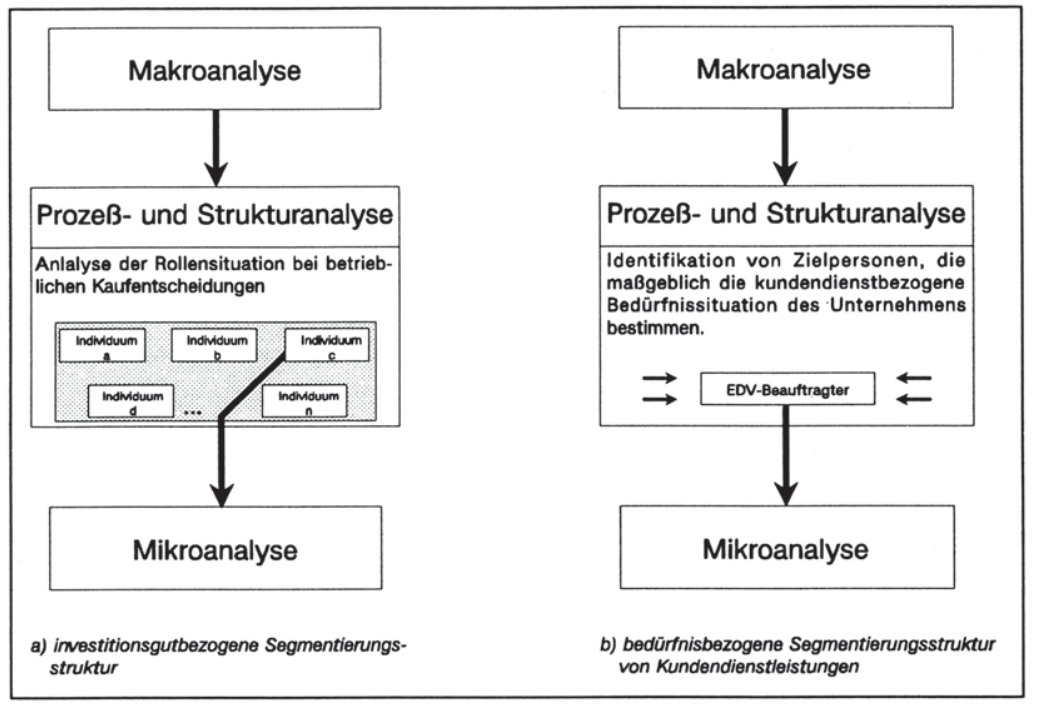

Abbildung 13: Strukturvergleich investitionsgut- und kundendienstbedürnisbezogener Segmentierungsprozesse 
gaben durch Offenlegung kaufverhaltensrelevanter Zusammenhänge als auch verfahrenstechnische Aufgaben in Form einer Filterfunktion für den weiteren Verlauf des Segmentierungsprozesses. Erst auf dieser Grundlage wird durch die Identifizierung prozeßdominanter Schlüsselpersonen eine weiterführende Analyse individueller Kennzeichen möglich. Abbildung 13 (Teil a) stellt diesen Zusammenhang schematisch dar.

Überträgt man diesen Sachverhalt auf die Anforderungen einer kundendienstbezogenen Bedürfnissegmentierung, so ergeben sich wesentliche Unterschiede. Hier sind die in Verbindung mit kundendienstpolitischen Überlegungen stehenden bedürfnisprägenden Ansprechpersonen im Unternehmen, die EDV-Beauftragten, a priori bekannt (Abbildung 13, Teil b).

Diese Hypothese basiert auf folgendem Hintergrund. Das generelle Kompetenzniveau einer Unternehmung in bezug auf PC-bezogene Sachverhalte stellt eine fiktive Bedarfsschwelle für Kundendienstleistungen dar. Treten Ausfälle und Störungen auf, die unterhalb dieses Niveaus liegen, besteht im Unternehmen die Möglichkeit, sich an einen externen Kundendienstleister zu wenden. Zusätzlich existiert die Alternative, die Ursachen durch Eigenleistung und Selbsthilfe zu beseitigen. Oberhalb dieser Schwelle ist die Vergabe derartiger Arbeiten an einen externen Serviceanbieter in der Regel jedoch unumgänglich (vgl. Abb. 14).

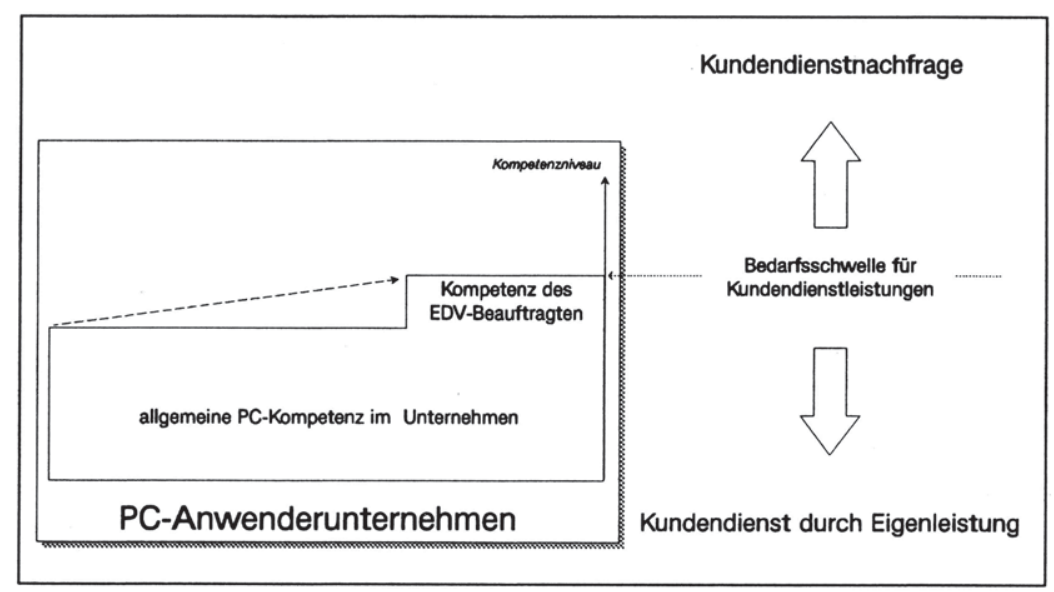

Abbildung 14: Bedarfsniveau für Kundendienstleistungen 
Obwohl sich die Unternehmenskompetenz zu PC-bezogenen Sachverhalten aus den Einzelkompetenzen der verschiedensten Mitarbeiter zusammensetzt, kann man doch unterstellen, daß der EDV-Beauftragte als Hauptleistungsverantwortlicher hierbei eine dominierende Stellung einnimmt. ${ }^{133}$ Seine tägliche Auseinandersetzung mit PC-bezogenen Aspekten läßt ebenso wie seine technologiesowie mitarbeiterbezogenen Erfahrung den Schluß zu, daß die kundendienstbezogene Bedürfnissituation seines Unternehmens maßgeblich durch sein Fachwissen dominiert und bestimmt ist. 134

Dieser Zusammenhang führt zu einer wesentlichen Vereinfachung des Segmentierungsprozesses. Die Filterfunktion der Prozeß- und Strukturanalyse entfältt bei servicebezogenen Aufgabenstellungen. ${ }^{135}$ Gemeinsam mit makroanalytischen Fragestellungen können mikroanalytische Gesichtspunkte parallel auf einer Segmentierungsebene analysiert werden. Damit reduziert sich der Prozeß einer kundendienstbezogenen Bedürfnissegmentierung gegenüber der des investitionsgüterbezogenen Modells zunächst auf eine Problemstufe. Im Ergebnis führt dies zu einem erheblich vereinfachten Bildungs- und Beschreibungsprozeß $\beta$ von kundendienstbezogenen Bedürfnissegmenten.

Die Prozeß- und Strukturanalyse gewinnt in einer zweiten Modellstufe der Kundendienstsegmentierung an Bedeutung. Unabhängig vom Einfluß des EDV-Beauftragten auf die kundendienstbezogenen Bedürfnisbildung gilt es, den Willenbildungs- und Entscheidungsprozeß zur Analyse kaufverhaltensrelevanter Machtstrukturen bei Kundendienstkäufen herauszuarbeiten. Hierdurch wird die Möglichkeit geschaffen, neben der bedürfnisbestimmenden Zielperson, die maßgeblichen Entscheidungsträger von Kundendienstkäufen in die Marktbearbeitungspolitik einzubeziehen. Damit wird der bislang fehlende Kaufentscheidungsbezug der Bedürfnisebene um den konkreten Nachfrageaspekt erweitert.

133 Die Unterstellung einer kompetenzführenden Stellung des EDV-Beauftragten im Vergleich zu anderen Mitarbeitergruppen im Unternehmen hat zunächst hypothetischen Charakter. Die Analyse der PC-bezogenen Kompetenzsituation in Unternehmen bildet daher auch einen ersten wichtigen Bestandteil der empirischen Analyse.

134 Dabei stellt das "Eigen-Know-how" des EDV-Beautragten lediglich eine Komponente seiner Kompetenz dar. Hinzu kommt das Wissen um, sowie die Vertügungsgewalt über das gesamte EDV-Know-how des Unternehmens.

135 Die grundsätzliche Institutionalisierung der EDV-Funktion, und damit die Voraussetzung einer eigenstăndigen EDV-bezogenen Verantwortlichkelt ist dabel zuvor im Rahmen der Markroanalyse zu prüfen. 
Im Gegensatz zu Wind/Cardozos Vorschlag dient die zweite Segmentierungsstufe der Kundendienstsegmentierung folglich vor allem zur Fundierung marktbearbeitungspolitischer Implikationen. Dem Charakter einer strategischen Marktsegmentierung wird hiermit in besonderer Weise entsprochen, indem neben der Marktaufteilung explizit marktbearbeitungsbezogene Aspekte einen Schwerpunkt bilden.

\subsection{Die Bildung bedarfsorientierter Kundendienstsegmente als primäre Problemstufe}

Die stellvertretend zur Messung von Kundendienstbedürfnissen herangezogenen Brückenkonstrukte "Abhängigkeitsgrad" und "Kundenkompetenz" bilden die Kerndimensionen der Marktaufteilung. Zunächst sind deshalb die Ausgangsvariablen in Beziehung zu diesen Brückenvariablen zu setzen und entsprechend zu systematisieren. Damit wird eine erste Voraussetzung zur Operationalisierung der Zusammenhänge erfüllt. Die Einflußfaktoren der beiden Kerndimensionen "Abhängigkeitsgrad" und "Kundenkompetenz" sind in Abbildung 15 zusammen

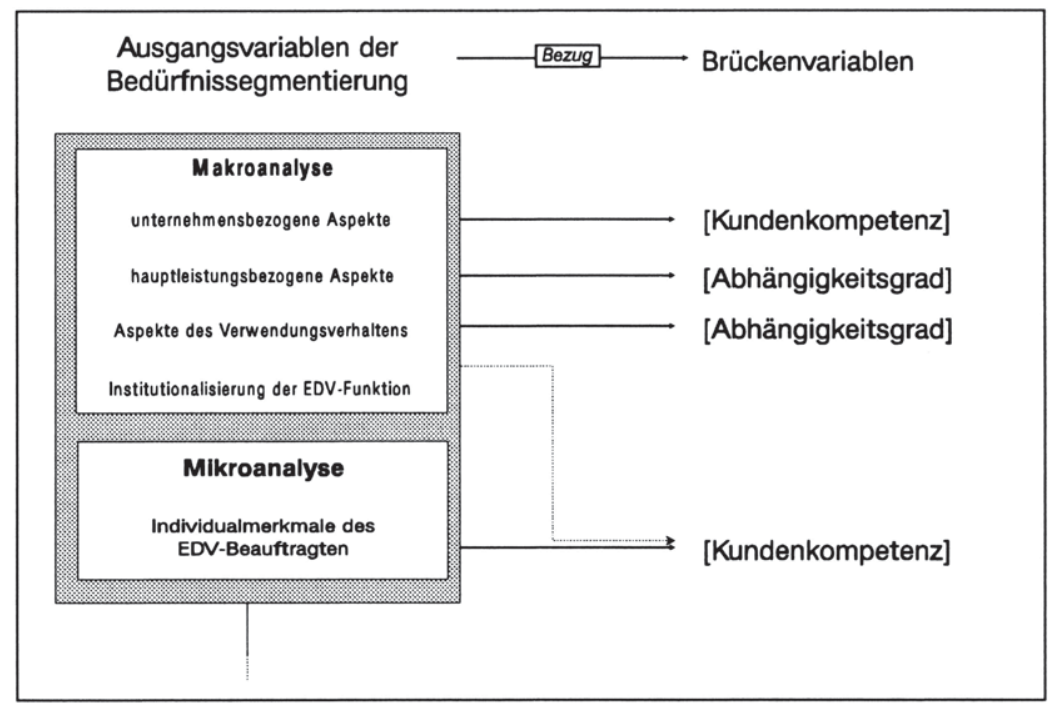

Abbildung 15: Zusammenhang zwischen Ausgangs- und Brückenvariablen im Rahmen der Bildung bedürfnisbezogener Kundendienstsegmente 
gefaßt. Aufgabe der empirischen Analyse ist es zu prüfen, ob und inwieweit diese unterstellten Zusammenhänge abbildbar sind und tatsächliche Segmentierungsrelevanz aufweisen.

Dem Abhängigkeitsgrad kann vor allem ein starker Bezug zu hauptleistungsund verwendungsbezogenen Einflüssen unterstellt werden. Die durch technische Ausstattungsmerkmale vorgegebenen Kapazitäten einer Hauptleistung bestimmen hierbei zunächst grundsätzlich deren Lösungsfähigkeiten und damit den theoretisch möglichen Abhängigkeitsgrad im Unternehmensprozeß. Allgemeine Beispiele hierfür sind Lade-, Produktions- oder Durchlaufkapazitäten, Ausschußraten, Motorleistung, Brennstoffverbrauch u.a. In bezug auf PC-Systeme ergeben sich besonders hardwarebezogene Restriktionen hinsichtlich der Speicherkapazităten und Verarbeitungsgeschwindigkeiten. Das tatsächliche Ausmaß der Einbindung in den Unternehmensprozeß wird durch verwendungsbezogene Kriterien festgelegt. Integrationsbreite und -tiefe bestimmen dabei den faktisch realisierten Abhängigkeitsgrad von einer Technologie. Hinzu kommt der Gesichtspunkt der Ausfallsensibilitāt. ${ }^{136}$

Die Kundenkompetenz steht vorwiegend in bezug zu unternehmensbezogenen und mikroanalytischen Aspekten. Dabei definiert sich insbesondere der generelle Erfahrungsstand im Umgang mit elektronischer Datenverarbeitung durch die erstgenannte Variablengruppe. Ausmaß und Breite der in der Organisation vorhandenen PC-Kompetenz werden dagegen maßgeblich durch individuelle Einschätzungen des EDV-Beauftragten erfaßt. ${ }^{137}$ Darüber hinaus tragen seine demographischen Kennzeichen, aber auch Einstellungen, Meinungen und Ansichten, die er im Rahmen seiner Stellung als EDV-Verantwortlicher stellvertretend für sein Unternehmen äußert, zum unternehmensbezogenen Kompetenzniveau bei.

136 Vgl. hierzu auch Kap B.2.21.

137 Vgl. hierzu auch Kap B.2.22. 


\subsection{Marktbearbeltungsorientierte Analyse kaufrelevanter Machtstruktu- ren als sekundäre Problemstufe}

Die Prozeß- und Strukturanalyse als zweite Segmentierungsstufe untersucht aufbauend auf den Ergebnissen der Bedarfsgruppenbildung die Machtstrukturen verschiedener Mitarbeiter in Kauf- und Entscheidungsprozessen bei Kundendienstkäufen. Die Analyse schafft damit zentrale Ansatzpunkte für die Gestaltung zielgruppengerechter Marktbearbeitungsmaßnahmen.

Obgleich durch die Kompetenz des EDV-Beauftragten in hohem Maße die kundendienstbezogene Bedürfnissituation eines Unternehmens geprägt ist, lassen sich hieraus noch keine unmittelbaren Schlüsse auf das tatsächliche Kauf- und Entscheidungsverhalten eines Unternehmens ziehen. Nur wenn der EDV-Beauftragte seine servicebezogenen Vorstellungen in Kaufentscheidungsprozessen auch tatsächlich durchsetzen kann, wandelt sich die Bedürfniskonstellation in konkrete Nachfrage. Anders gestaltet sich die Bereitschaft Service in Anspruch zu nehmen jedoch, wenn servicekonträre Interessen und Argumente anderer Unternehmensmitglieder dominieren. So ist z.B. durchaus vorstellbar, daß die Geschättsleitung eines Unternehmens letztendlich von den kundendienstbezogenen Vorstellungen und Ansprüchen ihres EDV-Beauftragten nicht überzeugt ist und das Budget für Serviceleistungen aufgrund von Kostensenkungsmaßnahmen reduziert oder sogar ganz streicht.

Somit sind für Kundendienstanbieter neben der segmentspezifischen Bedürfnislage auch jeweils die entsprechenden Entscheidungsprozesse und -strukturen von Bedeutung. Erst auf Grundlage dieser Kenntnisse läßt sich eine zielgruppengerechte Absatzpolitik implementieren.

Zur Analyse dieses Zusammenhangs bietet sich das Promotorenmodell von Witte an. ${ }^{138} \mathrm{Im}$ Rahmen einer empirischen Untersuchung teilt er die Mitglieder des Buying Centers in die zwei Gruppen der "Machtpromotoren" und "Fachpromotoren" ein. Promotoren sind dabei Mitarbeiter, welche die Kaufpro-

138 Vgl. Witte, E., Organisation für Innovationsentscheidungen - das Promotorenmodell, Göttingen 1973, S. 16 ff.; derselbe, Kraft und Gegenkraft im Entscheidungsprozeß, in: ZfB, 1976, Nr. 46, S. 324 f; Backhaus, K., Investitionsgütermarketing, a.a.O., S. 37 f.; Kern, E., Der Interaktionsansatz im Investitionsgütermarketing, a.a.O., S. 41 ff. 
zesse eines Unternehmens maßgeblich vorantreiben und prägen. Sie gelten als Schlüsselpersonen des betrieblichen Kaufverhaltens.

"Machtpromotoren" (meist Top-Manager) besitzen aufgrund ihrer hohen hierarchischen Position im Unternehmen zumeist die letztendliche Entscheidungsmacht. Ihr Votum hängt im wesentlichen vom Gesamteindruck des betrachteten Kaufgegenstands und seinen Auswirkungen auf die jeweiligen Unternehmensziele ab. ${ }^{139}$

"Fachpromotoren" zeichnen sich unabhängig von ihrer Position und Stellung im Unternehmen durch fach- bzw. objektspezifisches Detailwissen aus. Gerade bei sehr technologiebestimmten Kautprozessen werden Entscheidungen in hohem Maße durch den Einfluß dieser Gruppe getragen.

Neben Entscheidungsträgern und Meinungsbildnern ist eine dritte Gruppe in den zu betrachtenden Kaufentscheidungsprozessen von Bedeutung. Hierbei handelt es sich um Personen, die solche Prozesse überhaupt erst anregen bzw. initiieren. Bonoma bezeichnet diese Personen als "Initiatoren". 140

Bezūglich des vorliegenden Segmentierungsproblems bedeutet dies, besonders die Einflußnahme der EDV-Beauftragten auf kundendienstbezogene Entscheidungsprozesse zu ermitteln. Dabei ist zu überprüfen, ob sein bedürfnisbestimmender Einfluß auch seiner Einflußnahme auf das kundendienstbezogenen Kaufverhalten in Form eines "Initiators", "Fach-", und/oder "Machtpromotors" entspricht. Ferner ist die Bedeutung der Geschäftsleitung, der Abteilungs- und Bereichsleiter sowie der PC-Anwender für solche Kaufentscheidungen zu untersuchen. Im einzelnen ist hierzu die letztendliche Entscheidungsmacht, das Beeinflussungs- und Initiativerhalten dieser Gruppen zu analysieren. Eine derartige Charakterisierung des segmentspezifischen Kaufverhaltens offenbart nicht zuletzt gerade für eine zielgruppengerechte Kommunikationspolitik entscheidende Ansatzpunkte.

139 Meffert, H., Marketingforschung und Käuferverhalten, a.a.O., S. 145.

140 Vgl. Bonoma, T.V., Major Sales: Who Really Does the Buying?, in: Harvard Business Review, May/June 1982, S. $111 \mathrm{ff}$. 


\section{Bezugsrahmen und Hypothesen der Untersuchung}

Ausgehend von den diskutierten Einflußkriterien der kundendienstpolitischen Segmentierung sowie den theoretisch abgeleiteten Wirkungszusammenhängen wird der empirischen Untersuchung der in Abbildung 16 dargestellte Bezugsrahmen zugrunde gelegt. Die theoriegeleitete Auseinandersetzung mit den verschiedenen Gesichtspunkten der Marktsegmentierung hat ergeben, daß die Segmentierung von Kundendienstleistungen in einem zweistufigen Prozeß vollzogen werden kann.

Dabei wird mit Hilfe makro- und mikrobezogener Kriterien versucht die Basisdimensionen "Abhängigkeitsgrad" und "Kundenkompetenz" abzubilden, um so die Voraussetzung der bedürfnisbezogenen Aufteilung von Servicemärkten zu schaffen. Darüber hinaus erfolgt die Identifikation und Beschreibung einzelner Kundendienstsegmente.

Im Rahmen der Prozeß- und Strukturanalyse werden für die einzelnen Bedürfniscluster die Machtstrukturen der kundendienstbezogenen Kaufentscheidungsprozesse untersucht. Damit werden zentrale Voraussetzungen für die Entwicklung von Marktbearbeitungsmaßnahmen geschaffen.

Zielsetzung der empirischen Analyse ist es, das hergeleitete Konzept zur strategischen Marktsegmentierung von Kundendienstleistungen zu validieren und Einflußkriterien zu identifizieren, die zur Aufteilung, Beschreibung und Bearbeitung von Kundendienstmärkten beitragen. Hierzu werden die im Rahmen der theoriegeleiteten Diskussion vollzogenen Überlegungen in konkrete Untersuchungshypothesen überführt und in den Erklärungsansatz integriert.

Insgesamt sind drei Hypothesengruppen zu unterscheiden:

- Voraussetzungsbezogene Hypothesen (Hv) zur Überprüfung der dargelegten Grundannahmen zur Kundendienstsegmentierung:

HV1: Das EDV- und PC-Wesen von Unternehmen ist institutionalisiert. Damit besteht eine spezifische Verantwortlichkeit auch für kundendienstbezogene Fragestellungen. 


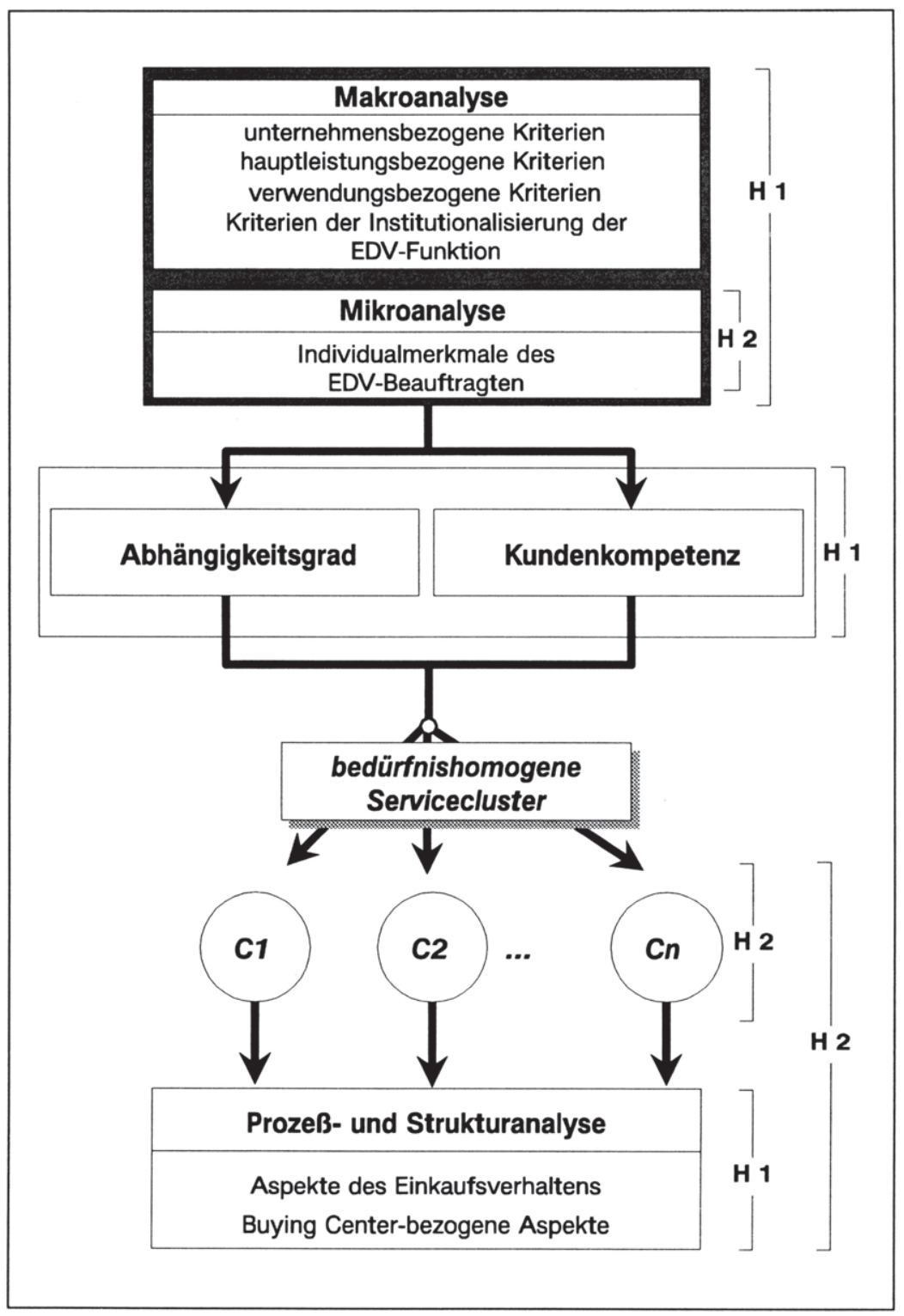

Abbildung 16: Bezugsrahmen der empirischen Untersuchung 
HV2: Der EDV-Beauftragte dominiert die EDV- bzw. PC-bezogene Kompetenz seines Unternehmens, d.h. gegenüber allen anderen Unternehmensmitgliedern besitzt er einen deutlichen Kompetenzvorsprung. Er stellt damit die Zielperson der Mikroanalyse dar.

- Segmentierungsbezogene Hypothesen $\left(\mathbf{H}_{\mathbf{S}}\right)$ zur Analyse zentraler Parameter und Zusammenhänge der kundendienstbezogenen Marktaufteilung:

HS1: PC-bezogene Kundendienstbedürfnisse eines Unternehmens lassen sich durch die Dimensionen "Abhängigkeitsgrad" und "Kundenkompetenz" abgrenzen. Beide Konstrukte stellen operationalisierbare Basisdimensionen einer bedürfnisbezogenen Marktaufteilung dar und bilden die Grundlage zur Identifikation verschiedenartiger Bedürfnissegmente im Kundendienstmarkt.

HS2: Im Markt für kommerzielle Personal Computer lassen sich trennscharfe Segmente mit jeweils homogenen Servicebedürfnissen identifizieren und beschreiben. Unterschiedliche Reaktionen auf Marketingimpulse von Kundendienstanbietern kennzeichnen dabei das Bild in den einzelnen Teilmärkten.

- Marktbearbeitungsbezogene Hypothesen $\left(\mathbf{H}_{\mathbf{M}}\right)$ zur Ermittlung teilmarktspezifischer Anforderungen für ein zielgruppengerechtes Kundendienstmarketing:

$\mathbf{H}_{\mathbf{M}}$ 1: Die Strukturen von kundendienstbezogenen Kaufentscheidungsprozessen sind vergleichbar zu denen von PC-Käufen. Die Nutzung hauptleistungsbezogener Erfahrungen führt von daher zur Erzielung wesentlicher Synergieeffekte im Rahmen der kundendienstbezogenen Marktbearbeitung.

$\mathbf{H}_{\mathbf{M}}$ 2: Die Machtstrukturen in bezug auf kundendienstbezogene Kaufentscheidungen sind segmentübergreifend unterschiedlich ausgeprägt. Innerhalb einzelner Bedürnissegmente lassen sich jedoch typische Entscheidungstrukturen identifizieren. 


\section{Empirische Analyse zur Segmentierung von Kundendienst- leistungen in investlven Märkten}

\section{Design der Untersuchung}

Die Überprüfung der zugrunde gelegten Untersuchungshypothesen zur Kundendienstsegmentierung erfolgt auf Grundlage einer 1991 durchgeführten Befragung bei 198 Unternehmen der mittelständischen Wirtschaft in den alten Bundesländern der Bundesrepublik Deutschland. Das Thema der Befragung lautete "Segmentierung von Kundendienstleistungen im kommerziellen PC-Markt". Vorrangiges Auswahlkriterium für die zu befragenden Unternehmen war der Einsatz von Personal Computern im Rahmen ihrer betrieblichen Datenverarbeitung.

Das wissenschaftlich vom Institut für Marketing der Universitāt Münster betreute und von der Firma Siemens Nixdorf Informationssysteme AG unterstützte Projekt wurde in Zusammenarbeit mit Infratest Industria realisiert.

Bevor die Untersuchung der einzelnen Hypothesen erfolgt, sollen zunächst die Auswahl- und Stukturparameter der durchgeführten Datenerhebung vorgestellt werden. Es wurden folgende weitere Anforderungen an die Stichprobenzusammensetzung gestellt:

- gleiche Besetzung aller drei Wirtschaftsbereiche Industrie, Handel und Dienstleistung

- Beschäftigtenzahl zwischen 20 und 1000 Mitarbeitern; im einzelnen:

- Industrie

$(100-1000 \mathrm{MA})$

- Handel

( 20 - $200 \mathrm{MA})$

- Dienstleistung

( 20 - $200 \mathrm{MA})$

- der PC-Einsatz sollte betrieben werden als:

- Insellösung (stand-alone PC) und/oder

- Anbindung an andere Mehrplatzsysteme und/oder

- PC-Netzbetrieb (direkte Verbindung mehrerer PCs untereinander).

In jedem Unternehmen wurden zwei persōnliche, standardisierte Interviews durchgeführt. Dabei wurden einem Mitglied der Geschäftsleitung vorwiegend Fragen zu unternehmensbezogenen Aspekten und zur generellen EDV-Situation gestellt. Der verantwortliche EDV-Beauftragte (-Zuständige) wurde in einem 
zweiten Gespräch vor allem zur spezifischen EDV/PC-Konfiguration im Unternehmen sowie zu individuellen demographischen und psychographischen Merkmalen befragt.

Bei beiden Personen wurden verschiedene Beantwortungskompetenzen unterstellt. Insofern verdichten sich pro befragtem Unternehmen beide Teilinterviews zu einer Gesamtstellungnahme. 141 Die Gesprächsdauer mit den Geschäftsführern lag zwischen 20 und 30 Minuten, während die Interviews mit den EDV-Beauftragten 30 - 40 Minuten in Anspruch nahmen.

Grundlage für alle Gespräche war ein zweiteiliger Fragebogen. Dabei umfaßt der erste Teil alle geschäftsführerbezogenen Fragen ("Fragen an ein Mitglied der Geschäftsführung"). Der zweite Komplex beinhaltet die an den EDV-Beauftragten gerichteten Fragen ("Fragen an den EDV-Verantwortlichen"). ${ }^{142}$

Den Schwerpunkt des standardisierten Fragebogens bildeten geschlossene Fragestellungen. 143 Zusätzlich wurden offene Antwortkategorien zur Erfassung unternehmensindividueller Besonderheiten integriert. Daneben wurden die Interviewer angewiesen, ergänzende Anmerkungen der Befragten schriftlich festzuhalten.

Angesichts der z.T. komplizierten aber unvermeidlichen Fachterminologie wurde ein Interviewleitfaden entwickelt, der bei Bedarf zu jeder Frage ausführliche Begriffsdefinitionen bereitstellte. Die Interviewer konnten somit bei Mißverständnissen oder Rückfragen entsprechende Erläuterungen geben. Damit wurde bei allen Gesprächen ein einheitliches Begriffsverständnis sichergestellt. 144

141396 Einzelinterviews führen somit zu einer Auswertungsbasis von 198 Unternehmensaussagen.

142 Vgl. hierzu die Fragebögen in Anhang II dieser Arbeit

143 Die Antworten wurden dabei vielfach mit Hilfe von Rating-Skalen erhoben. Der Vorteil dieser Vorgehensweise liegt darin, daß die Probanden ihre Aussagen differenzieren und abstufen können. Zur Befragungstaktik vgl. besonders: Meffert, $\mathrm{H}$., Marketingforschung und Käuferverhalten, a.a.O., S. $204 \mathrm{ff}$.

144 Die Erfahrung aus verschiedenen Expertengesprächen erbrachte wertvolle Anhaltspunkte zur Fragebogengestaltung. Darüber hinaus wurden beide Fragebogenteile sowie der Interviewleltfaden in bezug auf ihre Fragenlänge, -reihenfolge, -verständlichkeit und -formullerung in 15 Pretest-Interviews bei mittelständischen Unternehmen überprüft und optimiert. 
Die Befragungsinhalte leiten sich aus den diskutierten Kriterien des empirischen Bezugsrahmens der Kundendienstsegmentierung ab. Im Schwerpunkt wurden folgende Fragenkomplexe erfaßt: 145

- unternehmensdemographische Merkmale

- Kennzeichen und Bedeutung des EDV/PC-Betriebs

- PC-Ausstattung

- Kennzeichen PC- und kundendienstbezogener Kaufentscheidungsprozesse (Entscheidung, Beeinflussung, Anregung)

- Motive und Barrieren für die Inanspruchnahme von Kundendienstleistungen

- Integrationstiefe von PCs in einzelne Unternehmensbereiche

- Ausfallerfahrung und -sensibilität

- PC-bezogene Organisationskompetenz

- Bekanntheit und Bedeutung einzelner Serviceleistungen

- Erfahrungen und Zufriedenheit mit Serviceleistungen

- Schlüsselanforderungen an Kundendienstleister

- demographische und psychographische Kennzeichen des EDV-Beauftragten

Die statistische Datenauswertung erfolgte mit Hilfe des Softwarepaketes SPSS-X (Statistical Package for the Social Sciences), Version 3.0 für das Betriebssystem OS/2. ${ }^{146}$ Das vorliegende Datenmaterial weist vorwiegend metrisches Skalen-

145 Zum detaillierten Befragungskonzepts und der jewells konkreten Frageformulierung siehe Anhangs II.

$146 \mathrm{Zu}$ einem Überblick über die Möglichkeiten und Vorteile des Betriebssystems OS/2 vgl. Meder, N., Scheuber, P., MS-OS/2-Presentation-Manager: Einführung und Überblick, Haar bei München 1990, S. $15 \mathrm{ff}$. 
niveau auf. Ratingskalen wird unter der Prämisse gleicher Skalenabstände ein intervallskaliertes Meßniveau zugestanden. ${ }^{147}$

Neben univariaten Methoden sowie dem Verfahren der Indexbildung (Item Gesamtwert Analyse) kamen im Rahmen der Datenauswertung vor allem die multivariaten Analysemethoden der Faktoren-, Cluster- und Diskriminanzanalyse zur Anwendung. ${ }^{148}$ Bei den verwendeten Verfahren steht der forschungspragmatische Anwendungsbezug der einzelnen Verfahren im Vordergrund. Insofern wird auf eine statistisch-mathematische Detaildiskussion verzichtet.

Die Untersuchung von Mittelwertunterschieden verschiedener Gruppen erfolgte im Zwei-Gruppen-Fall mit Hilfe des T-Tests. 149 Bei Einzelunterschieden zwischen mehr als zwei Mittelwerten wurden Scheffé-Tests durchgeführt. 150

\section{Zentrale Voraussetzungen der Kundendienstsegmentierung}

\subsection{Die Einbindung der EDV-Funktion in die Organisation von Unter- nehmen}

Der kundendienstpolitische Segmentierungsansatz geht von der grundlegenden Voraussetzung aus, daß die Zuständigkeit und Verantwortlichkeit für PC-Sy-

147 Vgl. Backhaus, K., et al., Multivariate Analysemethoden, 6. Aufl., Berlin u.a. 1990, S. XIII.

148 Vgl. zu den einzelnen Prozeduren: Backhaus, K., et al., Multivariate Analysemethoden, a.a.O.; Meffert, H., Marketingforschung und Käuferverhalten, a.a.O.; Schubō, W., et al., Handbuch der Programmversion 4.0 und SPSS-X 3.0, Stuttgart, New York 1991; Steinhausen, D., Zörkendörfer, S., Statistische Datenanalyse mit dem Programmsystem SPSS-X und SPSS/PC ${ }^{+}$, 2. Aufl., München, Wien 1990; Kähler, W.-M., Statistische Datenanalyse mit SPSS/PC+ , Braunschweig 1990; Backhaus , K., Weiber, R., Entwicklung einer Marketingkonzeption mit SPSS/PC ${ }^{+}$, Berlin u.a. 1989.

149 Der T-Test ist ein Test- bzw. Prüfverfahren für metrisch skalierte Variablen, mit dem festgestellt werden kann, ob die Ereignisse der statistischen Datenauswertung signifikant sind. Vgl. hierzu Bleymüller, J., Gehlert, G., Gülicher, H., Statistik für Wirtschaftswissenschaften, 5. Auff., München 1988, S. $101 \mathrm{ff}$.

150 Die Anwendung des Scheffé-Tests bietet sich gegenüber anderen Mittelwertvergleichstests vor allem bei ungleichen Gruppengrößen an. Vgl. Bauer, F., Datenanalyse mit SPSS, Berlin u.a. 1984, S. 84 f.; Eimer, E., Varianzanalyse, Stuttgart u.a. 1979, S. 76 ff. 
steme in Unternehmen institutionalisiert ist. So baut nicht zuletzt die Zusammenführung von Makro- und Mikroanalyse auf der Annahme einer in der Unternehmung a priori bekannten und segmentierungsrelevanten Zielperson auf.

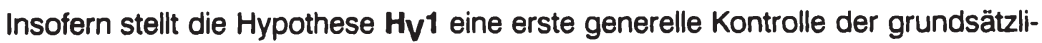
chen Tragfähigkeit des zugrunde gelegten Segmentierungsmodells dar. Sie wird wie folgt formuliert:

HV1: Das EDV- und PC-Wesen von Unternehmen ist institutionalisiert. Damit besteht eine spezifische Verantwortlichkeit auch für kundendienstbezogene Fragestellungen.

Die untersuchten Unternehmen wurden befragt, inwieweit sie ihr EDV-/PC-Wesen in die Organisationsstruktur integriert haben. Die Antwortmöglichkeiten "eigene" EDV-Abteilung" und "EDV-Beauftragte(r)" repräsentieren dabei den in der Hypothese geforderte Institutionalisierungsgrad. Die Antwort "weder noch" deutet darauf hin, daß das EDV-Wesen bislang keine explizite Berücksichtigung in seiner Behandlung erfahren hat (vgl. Abbildung 17).

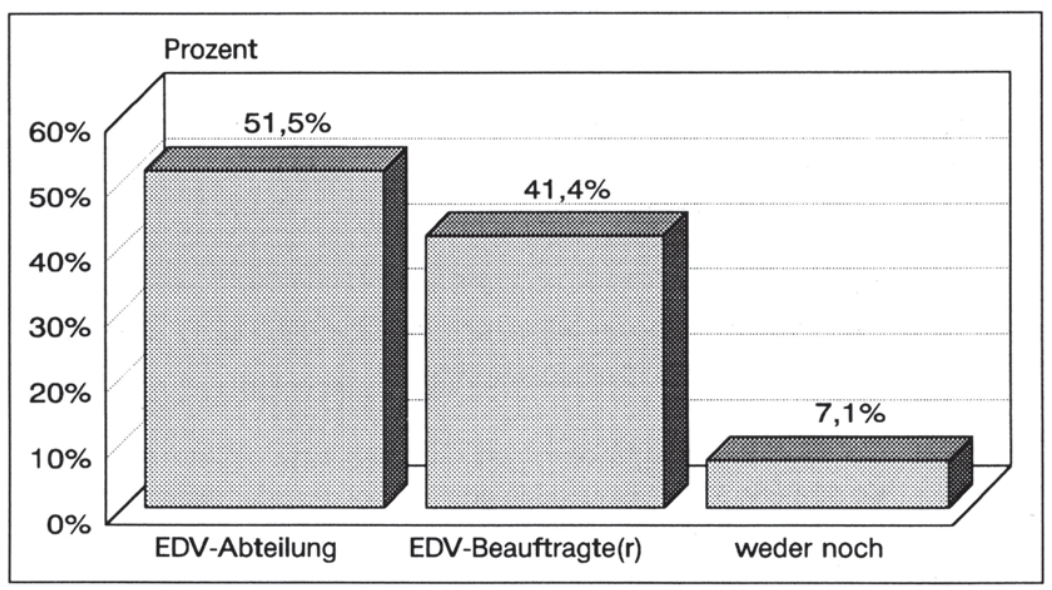

Abbildung 17: Institutionalisierung der EDV-Funktion

Die Analyse zeigt ein eindeutiges Bild. 92,9\% aller Unternehmen in der vorliegenden Stichprobe haben ihr EDV- und PC-Wesen institutionalisiert. Während $41,4 \%$ diese Aufgaben durch einen oder mehrere EDV-Beauftragte( $n)$ wahr- 
nehmen lassen, haben $51,5 \%$ der Unternehmen hierfür sogar eine eigene Abteilung geschaffen.

Wenngleich die Bestellung eines EDV-Beauftragten schon zu einer verantwortlichen Delegation EDV- und PC-bezogener Themen führt, läßt die Implementierung eigenständiger Abteilungen auf eine noch weiterführende Verankerung schließen. So zeigt sich etwa, daß $68,6 \%$ aller Unternehmen, die eine eigenständige EDV-Abteilung besitzen, dieser auch ein explizit, in ihren Planungsrechungen ausgewiesenes Budget zuordnen. In Unternehmen mit EDV-Beauftragten trifft dies nur noch in $35,4 \%$ aller Fälle zu.

Im Ergebnis kann damit festgestellt werden, daß die in Hypothese $\mathbf{H V}_{\mathbf{1}} \mathbf{\text { aufge- }}$ stellt Vermutung einer organisatorischen Verankerung EDV- und PC-bezogener Themen bestätigt ist. Annähernd $93 \%$ aller Unternehmen verfahren in solcher Weise, d. h. die Vermutung eines a priori bekannten und verfügbaren Ansprechpartners zur Durchführung einer Mikroanalyse triftt zu.

\subsection{Der EDV-Beauftragte als Kompetenzführer bei PC-bezogenen Auf- gabenstellungen}

Nachdem die grundsätzliche Frage der Existenz eines zuständigen Ansprechpartners beantwortet ist, bleibt zu prüfen, inwieweit diese Person in bezug auf PC-bezogene Themen kompetenzführend und somit bedürfnisprägend ist.

Dabei stellt sich vor allem die Frage, ob die Durchführung der Mikroanalyse mit der Person des EDV-Beauftragten tatsächlich tragfähig ist. Dieser Zusammenhang bestimmt die zweite Untersuchungshypothese:

HV2: Der EDV-Beauftragte dominiert die EDV- bzw. PC-bezogene Kompetenz seines Unternehmens, d.h. gegenüber allen anderen Unternehmensmitgliedern besitzt er einen deutlichen Kompetenzvorsprung. Er stellt damit die Zielperson der Mikroanalyse dar.

Zur Überprüfung dieses Aspektes ist die Kompetenz verschiedener Funktionsträger eines Unternehmens in bezug auf PC-bezogene Themen zu untersuchen. Hierzu wurde durch den EDV-Beauftragten jeweils das PC-bezogene Fachwis- 
sen der Geschäftsleitung, der Abteilungs- und Bereichsleiter (Produktion, Marketing, Rechnungswesen u.a.), der Anwender sowie das seiner eigenen Person mit Hilfe einer Statementbatterie beurteilt (vgl. Abbildung 18). ${ }^{151}$

Die Zusammenstellung der einzelnen Statements erfolgte hinsichtlich verschiedener Kompetenz-Aspekte. Neben der generellen Beurteilung wurde im einzelnen auf die Kompetenzdimensionen Aktualität, Intensität, Kreativität, Inhalte, Engagement und Synergien bezug genommen.

Das Ergebnisprofil weist eine deutliche Kompetenzüberlegenheit der EDV-Beaưtragten gegenüber den anderen Gruppen aus. Mit Ausnahme einer regelmäßigen Nutzung (zweite Position) dominieren sie alle angeführten Aspekte. Dabei streuen die Beurteilungen fast ausnahmslos um den zweithöchsten Skalenwert ("trifft zu") und weist insgesamt ein sehr konsistentes und positives Erscheinungsbild auf. Besonders die differenzierte Kenntnis der einzelnen Systemkomponenten Hardware, Software und Service ("trifft sehr zu") unterstreicht den aufgabenbezogenen Sachverstand dieser Personen.

Āhnlich konsistent fällt die Beurteilung der Geschäftsführer aus. Im Gegensatz zu den EDV-Beauftragten wird ihre PCKompetenz unter den betrachteten Gruppen jedoch am schlechtesten beurteilt. Ihr Fachwissen wird gering bis durchschnittlich eingestuft. Auffallend ist, daß sie in PCs offenbar eine reine Arbeitsplatztechnologie sehen. Anwendungen im privaten Bereich finden kaum statt. Auch im Beruf arbeiten Geschäftsführer vergleichsweise wenig mit diesen Systemen. Ursächlich hierfür mag sein, daß sie sich nur ungern mit PC-Technologie beschättigen.

Der Kompetenzstand der Anwender wird differenziert eingeschätzt. Dabei wird ihr Fachwissen vor allem in bezug auf die praktische Auseinandersetzung mit dieser Technologie besser beurteilt (arbeiten gerne und regelmäßig mit PCs/setzen diese zur Aưgabenlösung ein). Die Kompetenz dieser Gruppe ist damit überwiegend durch heuristische Kompetenzaspekte gekennzeichnet. ${ }^{152}$ Ein Wissen um Hintergrūnde bzw. um den Nutzen einzelner Systemkomponen-

151 Zugrunde gelegt wurde eine fünfstufige Skala. Dabei repräsentierten die Werte $1 \mathrm{mit}$ der Ausprăgung "trifft nicht zu" und 5 "trifft sehr zu" die beiden Extremwerte.

152

Vgl. Kap. B.2.22. 
ten besteht weniger. Insofern ist die PC-bezogene Fachkompetenz dieser Gruppe eher anwendungsorientiert und damit rein operativ einzustufen.

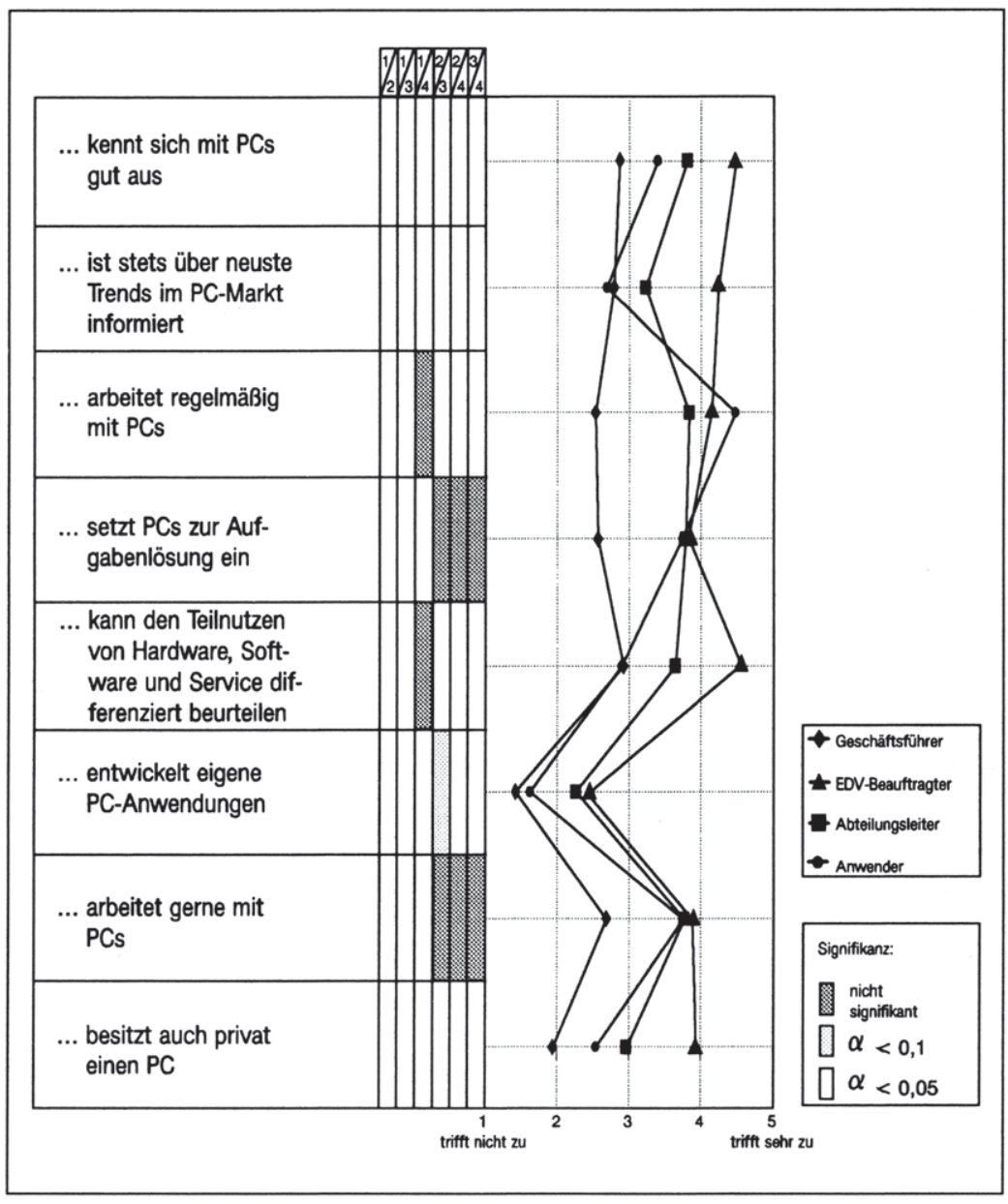

Abbildung 18: PC-Kompetenz verschiedener Funktionsträger

Das Wissen der Abteilungsleiter um PC-bezogene Zusammenhänge ist relativ gut. Obgleich sie im Gesamtbild nicht die hohe Bewertung der EDV-Beauftragten erreichen, weisen sie gegenüber den beiden anderen Personengruppen einen 
deutlich höheren Sachverstand auf. Dies betrifft sowohl die praktische Anwendung von PCs, als auch die Auseinandersetzung mit Vor- und Nachteilen einzelner Systemkomponenten.

Eine zusammenfassende Darstellung der Kompetenzsituation in Unternehmen zeigt Abbildung 19. Hierzu wurden sämtliche Einzelindikatoren der Kompetenz jeweils personenbezogen zu einem Index verdichtet. 153

Zuvor wurde dabei die innere Konsistenz der gebildeten vier Kompetenzindizes analysiert. Diese Überprüfung erfolgt mit Hilfe der Kennzahl Cronbach's Alpha. ${ }^{154}$ Dieses Maß kann Werte zwischen 0 und 1 annehmen, wobei bei einem Koeffizienten von 1 eine vollständige Konsistenz des Index gegeben ist. 155 Für praktische Anwendungen wird üblicherweise ein Grenzwert von 0,7 als akzeptables Ergebnis angenommen. ${ }^{156} \mathrm{Im}$ vorliegenden Fall überschritten alle vier Kompetenzindizes diese Schwelle und können somit als hinreichend konsistent angesehen werden. 157

Das Ergebnis der gruppenbezogenen Kompetenzbetrachtung unterstreicht deutlich die dominierende Stellung des EDV-Beauftragten. Aus ihrem überlegenen Sachverstand läßt sich damit die umfassende Kenntnis der kundendienstbezogenen Bedürfnissituation ihres Unternehmens ableiten. Ein insgesamt durchschnittliches Know how weisen Abteilungsleiter gefolgt von den PC-Anwendern auf. Mit einer geringen PC-Kompetenz und deutlichem Abstand zu den anderen Gruppen liegen die Geschäftsführer an letzter Stelle.

153 Ein einfacher Index aus $n$ Variablen wird gebildet, indem die Summe der Merkmalsausprägungen durch $n$ dividiert wird (arithmetisches Mittel), wobei die einzelnen Skalen zuvor zu normieren sind. Sollen einzelne Statements einer Batterie stärker betont bzw. herausgestellt werden, kann durch die Hinzunahme von Gewichtungsfaktoren eine zusätzliche Verfeinerung erfolgen. Hiervon wurde in der vorliegenden Situation jedoch abgesehen. $\mathrm{Zu}$ einer ausführlichen Darstellung dieses Verfahrens vgl. Bauer, F., Datenanalyse mit SPSS, a.a.O., S. $247 \mathrm{ff}$.

154 Cronbach's Alpha gilt als Maß für die innere Konsistenz eines Index und kann mit Hilfe der SPSS-Prozedur RELLABILTY berechnet werden. Vgl. Ebenda, S. 256.

155 Vgl. hierzu Lienert, G.A., Testaufbau und Testanalyse, 3. Auff., Weinheim 1969, S. 220 ff.

156 Vgl. Bauer, F., Datenanalyse mit SPSS, a.a.O., S. 256.

157 Im einzelnen ergaben sich folgende Cronbach's Alpha Werte: Geschäftsführerkompetenz 0,9015, Kompetenz des EDV-Beauftragten 0,8374, Abteilungsleiterkompetenz 0,8806 und Anwenderkompetenz 0,7530 . 


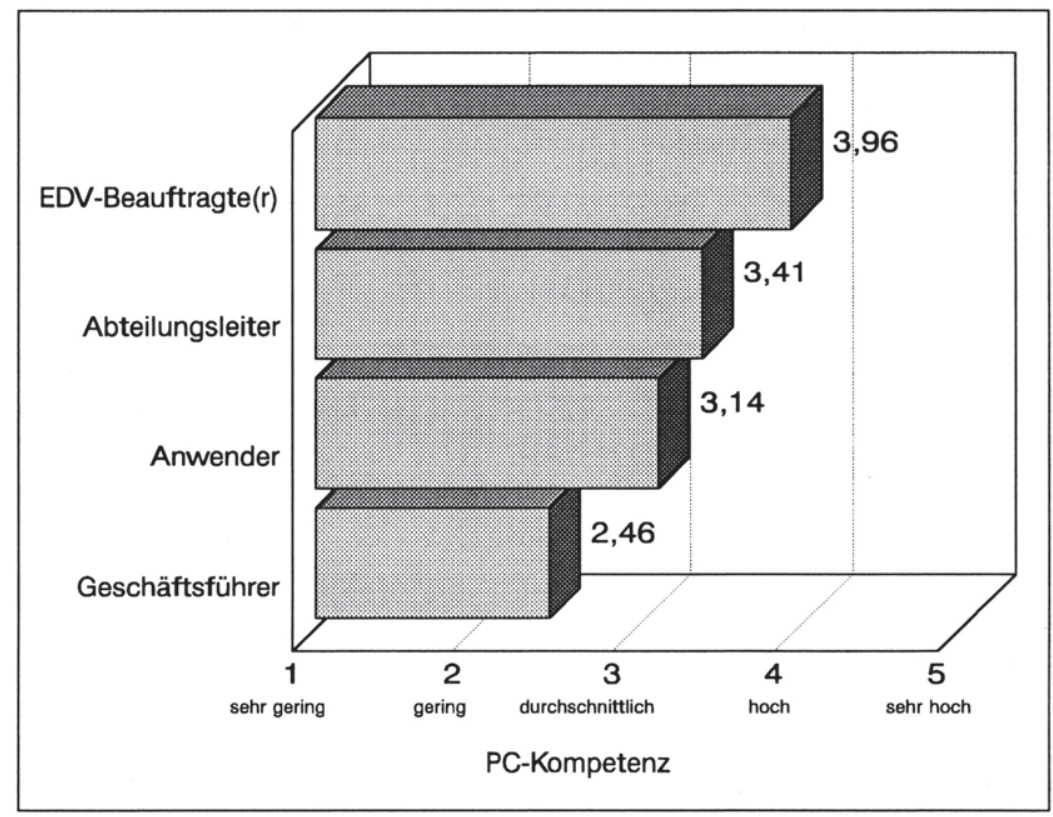

Abbildung 19: PC-bezogene Gruppenkompetenz

Die Analyse bestätigt somit die in Hypothese HV2 aufgestellte Vermutung. Die EDV-Beauftragen sind in bezug auf PC-bezogene Aspekte kompetenztührend und determinieren somit in entscheidendem $\mathrm{Maß \theta}$ die kundendienstspezifischen Anforderungen und Vorstellungen ihrer Unternehmen. Damit ist eine Durchführung der Mikroanalyse mit dieser Personengruppe gerechtfertigt und folgerichtig. 


\section{Die Bildung bedarfsorientierter Kundendienstsegmente im Markt für kommerzielle Personal Computer}

\subsection{Die Bildung kundendienstbezogener Kerndimensionen}

Zur Identifikation unterschiedlicher Bedürfnismuster im Kundendienstmarkt für Personal Computer gilt es nun zunächst die beiden Basisdimensionen "Abhängigkeitsgrad" und "Kundenkompetenz" zu operationalisieren. Beide sollen zusammen die zentralen Parameter einer Aufteilung von Servicemärkten darstellen. Die erste Untersuchungshypothese zur Marktaufteilung kann damit wie folgt formuliert werden:

Hs1: $\quad$ PC-bezogene Kundendienstbedürnisse eines Unternehmens lassen sich durch die Dimensionen "Abhängigkeitsgrad" und "Kundenkompetenz" abgrenzen. Beide Konstrukte stellen operationalisierbare Basisdimensionen einer bedürfnisbezogenen Marktaufteilung dar und bilden die Grundlage zur Identifikation verschiedenartiger Bedürfnissegmente im Kundendienstmarkt.

Da bislang auf keine vergleichbaren Untersuchungen zur Segmentierung von Kundendienstleistungen zurückgegriffen werden kann, steht die Ermittlung valider Faktoren zur Kennzeichnung dieser beiden Argumente im Vordergrund dieses Untersuchungsschrittes.

\subsection{Der Abhängigkeitsgrad}

Vor Beginn der weiteren Variablenverdichtung zu zentralen Abhāngigkeitsfaktoren soll zunächst die generelle Einbindung von Personal Computern in verschiedenen Unternehmensbereichen dargestellt werden (vgl. Abbildung 20).

Insgesamt ergibt sich derzeit eine noch eher geringe PC-Abhängigkeit in Unternehmen. 158 Überdurchschnittlich eingebunden sind diese Systeme bislang lediglich im Büro- und Sekretariatswesen sowie in rechnungs- und kalkulationsorientierten Unternehmensbereichen. Dagegen hat eine PC-Verknūpfung mit Auf-

158 Vgl. hierzu auch Kap. C.5. 
gaben von produktionsnahen Abteilungen (Qualitätswesen, Fertigungsorganisation, F\&E, Entwurf) bis dato kaum stattgefunden.

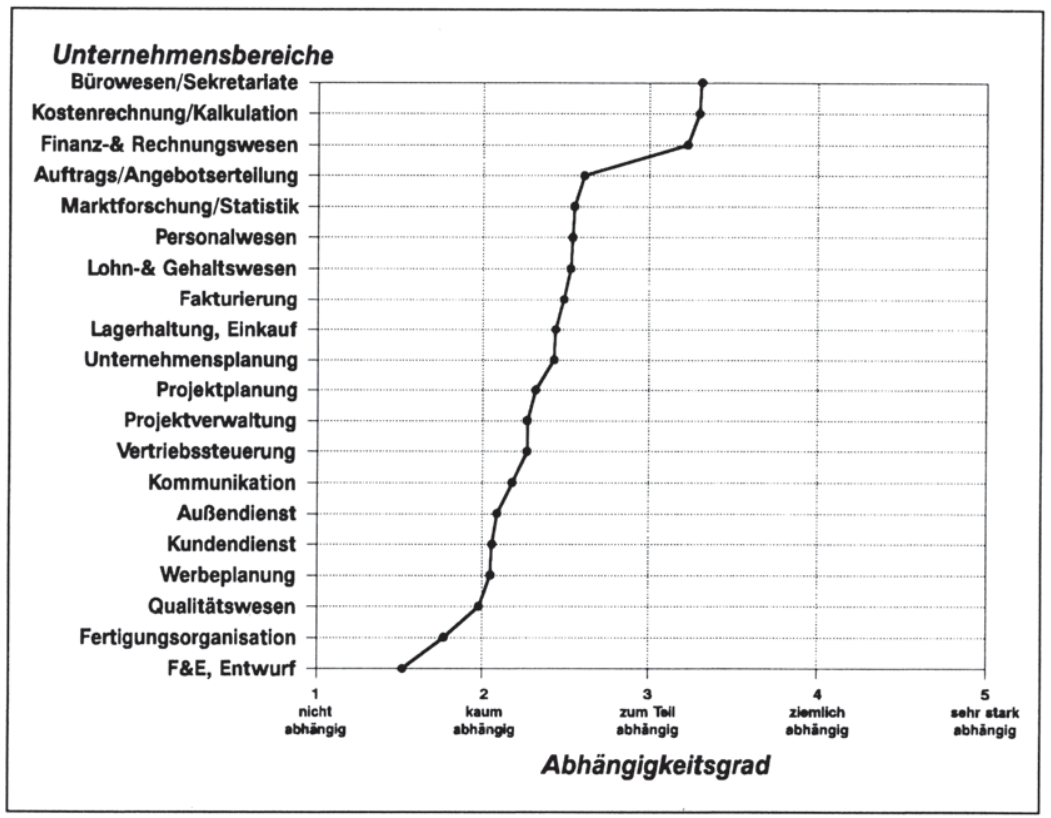

Abbildung 20: PC-Abhängigkeit in verschiedenen Unternehmensbereichen

Ausgehend von der sehr differenzierten Perspektive abteilungsbezogener PC-Integration erfolgt eine Reduktion der bereichsbezogenen Abhängigkeiten auf zentrale Faktoren. Zusätzlich finden Variablen der Ausfallsensibilität Berücksichtigung. 159 Damit werden sämtliche zur Verfügung stehenden abhängigkeitsbezogenen Ausgangsvariablen im Verlauf der weiteren Analyse berücksichtigt.

Unter Einbeziehung von 22 Ausgangsvariablen wird mit Hilfe einer explorativen Faktorenanalyse eine Datenverdichtung durchgeführt. Unter Verwendung des Kaiser-Kriteriums können dabei sechs Faktoren extrahiert werden, mit denen die

159 Die Ausfallsensibilität wurde anhand akzeptabler Ausfallzeiten bei defekten Systemen gemessen. Auch diese Einschätzung erfolgte anhand fünfstufiger Ratingskalen. 
PC-bezogene Abhängigkeit in Unternehmen charakterisiert werden kann. 160 Die Faktoren erklären einen gemeinsamen Varianzanteil von $61,8 \%$. Der damit verbundene Informationsverlust liegt im Rahmen vergleichbarer Untersuchungen. 161

Der Erklärungswert der gefundenen Faktoren ist auch vor dem Hintergrund der zugrundeliegenden Kommunalitäten zu beurteilen. Sie beeinflussen das Ergebnis einer Faktorenanalyse erheblich. Kommunalitäten geben an, wieviel Prozent der Varianz einer einzelnen Variablen durch die extrahierten Faktoren erklärt werden sollen. ${ }^{162} \mathrm{Im}$ vorliegenden Fall findet insgesamt eine hinreichende $\left(h_{i}{ }^{2}\right.$ $>0,5$ ) Varianzdarlegung der einzelnen Ausgangsgrößen statt. Lediglich die Variablen "Bürowesen/Sekretariate" und "Auftrags-/Angebotserteilung" werden mit Werten von 0,34 und 0,48 nur zu einem geringeren Teil von den gemeinsamen Faktoren erklärt.

Zur Faktorinterpretation werden Variablen mit einer Faktorladung von mindestens 0,5 herangezogen. Eine Ausnahme stellen "Vertriebsplanung", "Kundendienst" und "Bürowesen/Sekretariate" dar. Ihre Grenzwerte liegen geringfügig niedriger, finden aber dennoch entsprechende Berücksichtigung. 163 Insgesamt ergibt sich ein sehr gut interpretierbares Bild. Drei Indikatoren weisen mit Faktorladungen $\left(a_{i}\right)$ von $a_{i}>0,4$ Doppelladungen auf, woraus jedoch keiner-

160 Nach dem Kaiser-Kriterium werden nur Faktoren mit einem Eigenwert größer als 1 generiert. Der Eigenwert eines Faktores erklärt dessen Anteil an der Varianz aller Einzelvariablen. Als Schätzverfahren wurde in den vorliegenden Untersuchungen die Hauptkomponentenmethode benutzt. Zur besseren Interpretation der extrahierten Faktoren wurde eine VarimaxRotation durchgeführt. Zur Faktorenanalyse vgl. U.a.: Backhaus, K., et. al., Multivariate Analysemethoden, a.a.O., S. 67 ff.; Meffert, H., Marketingforschung und Käuferverhalten, a.a.O., S. 257 H.

161 Vgl. Kirchgeorg, M., Ökologieorientiertes Unternehmensverhalten - Typologien und Erklärungsansätze aư empirischer Grundlage, Wiesbaden 1989, S. 138 f.; Büker, B., Qualitätsbeurteilung investiver Dlenstleistungen, a.a.O., S. 124.; Ostmeier, H., Ókologieorientierte Produktinnovationen, Frankłurt/M, Bern, New York, Paris 1990, S. 56.; Poggenpohl, M., Verbundanalyse im Einzelhandel auf Grundlage von Kundenkarteninformationen. Eine empirische Untersuchung von Verbundbeziehungen zwischen Abteilungen, (unveröffentlichtes Manuskript), Münster 1993, S. 159.

162 Vgl. Backhaus, K., et al., Multwariate Analysemethoden, a.a.O., S. 99.

163 Nach Backhaus stellt dleser Grenzwert eine bewährte Konvention dar. Er betont in diesem Zusammenhang jedoch, daß zur Faktorinterpretation alle Varlablen ab einer Ladung von 0,5 hinzuzuziehen sind. Vgl. Ebenda, S. 92. 
lei Interpretationsprobleme resultieren. ${ }^{164}$ Die Faktorenstruktur ist in Tabelle 2 dargestellt.

Faktor 1 wird hoch geladen von einer PC-Abhängigkeit in den Unternehmensbereichen Fakturierung, Lagerhaltung/Einkauf, Finanz- und Rechnungswesen, Auftrags- und Angebotserteilung sowie dem Kundendienst. Alle Aufgaben in diesen Bereichen werden maßgeblich durch administrative, kaufmännische Tätigkeiten bestimmt. Demzufolge kann dieser Faktor als "PC-ABHÄNGIGKEIT IM KAUFMÄNNISCHEN WESEN" interpretiert werden.

Der zweite Faktor setzt sich aus einer Systemabhängigkeit in der Unternehmensplanung, Projektplanung und -verwaltung, Kostenrechung/Kalkulation, Marktforschung/Statistik, Werbeplanung und mit gewissem Abstand der Vertriebsplanung zusammen. Alle Bereiche kennzeichnen gemeinsam die Aufgaben und das Wesen eines modernen Marketing. Demzufolge wird Faktor 2 als "PCABHÄNGIGKEIT IM MARKETING-WESEN" definiert.

Faktor 3 wird durch eine PC-Abhängigkeit in den Abteilungen Qualitätswesen, Fertigungsorganisation sowie Forschung \& Entwicklung/Entwurf beschrieben. Angefangen vom Entwurf und der Entwicklung , über die eigentliche Fertigung bis hin zur Qualitätsprüfung sind alle drei Ressorts in hohem Maße produktbezogen. Somit kann dieser Faktor als "PC-ABHÄNGIGKEIT IM PRODUKTWESEN" bezeichnet werden.

Faktor 4 zeigt eine hohe Ladung im Bereich des Außendienst- sowie Kommunikationswesens. Mit größerem Abstand zeigt sich auch noch ein gewisser Einfluß auf den Kundendienst, die Vertriebsplanung sowie das Büro- und Sekretariatswesen. Dieses Bild spiegelt eine starke Bedeutung des mobilen PC-Einsatzes, wie man inn z.B. vielfach im Versicherungswesen findet, wider. Faktor 4 wird deshalb als "PC-ABHÄNGIGKEIT IM AUBENDIENSTWESEN" festgelegt.

Der fünfte Faktor setzt sich aus den Indikatoren der Ausfallsensibilität bei weniger wichtigen und wichtigen PC-Systemen zusammen. Die hohen Faktorladungen mit Werten von 0,895 und 0,869 lassen auf einen sehr stabilen Faktor schließen, der als "PC-BEZOGENE AUSFALLSENSIBILITÄT" interpretiert werden kann.

164 Vgl. hierzu die Variablen "Kostenrechnung/Kalkulation", "Vertriebsplanung" und "Lohn/Gehaltsabrechnung" und Tabelle 2. 


\begin{tabular}{|c|c|c|c|c|c|c|}
\hline Indikatoren des Abhängigkeitsgrads & Faktor 1 & Faktor 2 & Faktor 3 & Faktor 4 & Faktor 5 & Faktor 6 \\
\hline Fakturierung & 0,753 & $-0,058$ & 0,009 & 0,044 & 0,047 & 0,263 \\
\hline Lagerhaltung/Einkauf & 0,749 & 0,025 & 0,164 & 0,013 & 0,054 & 0,054 \\
\hline Finanz-/Rechnungswesen & 0,702 & 0,132 & $-0,043$ & 0,122 & $-0,032$ & 0,285 \\
\hline Auftrags-/Angebotserteilung & 0,651 & 0,075 & 0,145 & 0,161 & 0,082 & 0,022 \\
\hline Kundendienst & 0.503 & 0.163 & 0,290 & 0,398 & 0,159 & 0,065 \\
\hline Unternehmensplanung & 0,056 & 0,736 & 0,013 & 0,080 & 0,275 & 0,149 \\
\hline Projektplanung & $-0,124$ & 0,726 & 0,336 & $-0,018$ & 0,043 & 0,142 \\
\hline Projektverwaltung & 0,115 & 0,648 & 0,357 & 0,025 & 0,032 & 0,142 \\
\hline Kostenrechnung/Kalkulation & 0,515 & 0,588 & $-0,148$ & $-0,041$ & $-0,050$ & 0,060 \\
\hline Marktforschung/Statistik & 0,353 & 0,555 & 0,036 & 0,243 & $-0,082$ & $-0,363$ \\
\hline Werbeplanung & $-0,022$ & 0,544 & $-0,030$ & 0,224 & $-0,253$ & 0,061 \\
\hline Vertriebsplanung & 0,422 & 0,447 & 0,213 & 0,361 & 0,094 & $-0,059$ \\
\hline Qualitätswesen & 0,059 & 0,018 & 0,803 & 0.126 & 0,023 & 0,130 \\
\hline Fertigungsorganisation & 0,176 & 0,084 & 0,684 & $-0,011$ & $-0,071$ & $-0,033$ \\
\hline F\&E/Entwurf & 0,036 & 0,255 & 0,672 & 0,130 & $-0,071$ & 0,008 \\
\hline Außendienst & 0,120 & 0,069 & 0,160 & 0,828 & 0,045 & $-0,039$ \\
\hline Kommunikation & 0,150 & 0,021 & $-0,019$ & 0,622 & 0,095 & 0,313 \\
\hline Bürowesen/Sekretariate & 0,057 & 0,380 & 0,114 & 0,395 & -0.009 & $-0,140$ \\
\hline Ausfallsensibilitāt b. niedriger PC-Bedeutung & 0,085 & 0,003 & $-0,060$ & $-0,002$ & 0,895 & 0,007 \\
\hline Ausfallsensibilität $b$. hoher PC-Bedeutung & 0,062 & 0,100 & $-0,045$ & 0,104 & 0,869 & 0.024 \\
\hline Personalwesen & 0,223 & 0,144 & 0,071 & 0,195 & $-0,039$ & 0,775 \\
\hline Lohn-/Gehaltsabrechnung & 0,432 & 0,111 & 0,094 & $-0,072$ & 0,030 & 0,761 \\
\hline Erklārter Varianzantell $=61,8 \%$ & $24,9 \%$ & $10,7 \%$ & $8,1 \%$ & $6,7 \%$ & $6,2 \%$ & $5,2 \%$ \\
\hline
\end{tabular}

Tabelle 2: Faktoranalytisch verdichtete Dimensionen des Abhängigkeitsgrades von Personal Computern 
Faktor 6 schließlich weist starke Ladungen der Variablen PC-Abhängigkeit im Personalwesen sowie in der Lohn- und Gehaltsabrechung auf. Aufgrund der starken Orientierung an personalbezogenen Aufgaben läßt sich dieser Zusammenhang als "PC-ABHÄNGIGKEIT IM PERSONALWESEN" beschreiben.

Insgesamt lassen sich damit die beschriebenen Zusammenhänge einer unternehmensbezogenen PC-Abhängigkeit durch diese sechs Faktoren ausreichend darstellen und charakterisieren.

\subsection{Die Kundenkompetenz}

Die auswertungstechnische Vorgehensweise zur Analyse des zweiten Brückenkonstrukts, der Kundenkompetenz, erfolgt im wesentlichen analog zu der des Abhängigkeitsgrads. Auch hier findet eine Reduktion der Ausgangsvariablen auf zentrale Kompetenzparameter mit Hilfe einer explorativen Faktorenanalyse statt.

Alle zur Verfügung stehenden subjektiven Kompetenzurteile werden zur Verdichtung dieses Kompetenzkonstruktes herangezogen. 165 Dies umfaßt im einzeinen beschreibende Fertigkeiten und Fähigkeiten unterschiedlicher Mitarbeitergruppen in Unternehmen. 166 Darüber hinaus bilden die bisherigen Erfahrungen mit PC-bezogenen Ausfall- und Störsituationen eine Komponente der subjektiv bestimmten PC-Kompetenz. So führen Ausfallerfahrungen im Laufe der Zeit zu erfahrungsbestimmten Möglichkeiten der Selbsthilfe. 167 Neben der Vertrautheit mit Problemsituationen stellen schließlich die generellen bisherigen Erfahrungen von Unternehmen mit EDV-Systemen bzw. speziell PC-Technologien weitere aufzunehmende Variablen dar.

Insgesamt bilden damit 35 Variablen das Ausgangsmaterial der anstehenden Faktorenanalyse. Das Verfahren errechnet unter Verwendung des Kaiser-Kriteriums eine 9-Faktoren Lösung zur Kennzeichnung der Kundenkompetenz. Der gemeinsam erklärte Varianzanteil dieses Ergebnisses liegt mit 68,1\% über dem

165 Vgl. hierzu auch Kap. B.2.22.

166 Vgl. hierzu auch Kap. C.2.2, insb. Abbildung 18.

167 Vgl. Stäudel, Th., Problemlösen, Emotionen und Kompetenz, a.a.O., S. 55. 
Resultat der Abhängigkeitsanalyse und stellt ein hinreichend gutes Ergebnis dar. 168

Auch die Kommunalitätenstruktur ist zufriedenstellend. Bei 34 Variablen liegt der durch die gemeinsamen Faktoren erklärte Varianzanteil über 0,5. Lediglich die Variable "Anwender arbeiten regelmäßig mit PCs" wird nur zu 42,9\% berücksichtigt.

Die Interpretation der Ergebnisse erfolgt anhand der in Tabelle 3 dargestellten Faktorladungen. Zur Faktordeutung werden wiederum Variablen ab einer Ladung von 0,5 herangezogen. Bei inhaltich offensichtlichen und plausiblen Zusammenhāngen wurden in Einzelfällen auch noch Faktorladungen mit einer Mindestgröße von 0,4 berücksichtigt. 169 Trotz z.T. bestehender Doppelladungen ergibt sich ein substantiell klar interpretierbares Bild.

Faktor 1 beinhaltet sämtliche PC-bezogenen Kompetenzmerkmale des Geschäftsführers. Dies betrifft zum einen anwendungsorientierte bzw. operative Gesichtspunkte der praktischen Auseinandersetzung mit PCs (arbeitet gerne und regelmäßig mit PCs, kennt sich gut aus, benutzt PCs zur Aufgabenlōsung, private Nutzung). Zum anderen bestehen hohe Faktorladungen bei eher theoriespezifischen Kompetenzvariablen, d.h. bei Aspekten, die PC-bezogenes Hintergrundwissen dokumentieren (über neuste PC-Trends informiert, entwickeln eigene Anwendungen, können Teilnutzen beurteilen). Insgesamt kennzeichnet Faktor 1 damit die generelle Bereitschaft des Geschäftsführers, sich mit PC-bezogenen Sachverhalten auseinanderzusetzen. Ein derartiges Engagement läßt sich auch treffend als Involvement beschreiben. 170 Faktor 1 wird somit als "PCINVOLVEMENT DES GESCHÄFTSFÜHRERS" definiert.

168 Vergleichbar zur erhebungstechnischen Verfahrensweise bei den anderen Kompetenzvariablen, wurden auch die EDV- und PC-bezogenen Ertahrungsvariablen (urspünglich: verhältnisskallert gemessen) auf ein fünfstufiges Skalenniveau (intervallskallert) umcodiert. Vgl. hierzu im Anhang II dieser Arbelt die Fragen 21 und 27 im Fragebogen des EDV-Beauttragten sowie die Frage 5 (EDV und PC) im Geschäftsführerfragebogen.

169 Derart berücksichtigte Grenzwerte sind in Tabelle 3 besonders gekennzeichnet. Vgl. hierzu Tabelle 3, insbesondere zur Interpretation der Faktoren 6 und 8.

170 Meffert definiert Involvement als inneren Zustand der Aktivierung, der "in Abhängigkeit von der persönlichen Relevanz beim Individuum unterschiedliche Wirkungen auf Informationsaufnahme, -verarbeltung und -speicherung auslöst". Meffert, H., Marketingforschung und Kăuferverhalten, a.a.O., S. 66. 
Gefü können Teilnutzen von PC-Komponenten bewerten Gefũ entwickeln eigene Arwendungen

GeFũ benutzen auch im privaten Bereich PCs

AbtL kennen sich mit PCs gut aus

AbtL arbeiten regelmäBig mit PCs

AbtL sind stets über neuste PC-Trends informiert

AbtL setzen PCs zur Aufgabenlósung ein

AbtL kōnnen Teilnutzen von PC-Komponenten bewerten

AbtL arbeiten gerne mit PCs

AbtL entwickeln eigene Arwwendungen

AbtL benutzen auch im privaten Bereich PCs

EDVB arbeitet regelmäBig mit PCs

EDVB setzt PCs zur Aufgabenlósung ein

EDVB kann Teilnutzen von PC-Komponenten bewerte

EOVB kennt sich mit PCs gut aus

EDVB arbeitet gerne mit PCs

EDVB benutzt auch im privaten Bereich PCs

EDVB sind stets über neuste PC-Trends informiert

EDVB entwickelt eigene Anwendungen

Anwe kennen sich mit PCs gut aus

Arnwe sind stets über neuste PC-Trends informiert

Amwe kōnnen Teilnutzen von PC-Komponenten bewerten

Arwwe setzen PCs zur Autgabenlösung ein

Anwe arbeiten gerne mit PCs

Anwe arbeiten regelmäßig mit PCs

Anwe benutzen auch im privaten Bereich PCs

Anwe entwickeln eigene Arwendungen

PC-Erfahrung im Unternehmen

EDV-Erfahrung im Unternehmen

Stör- und Ausfallerfahrungen

\begin{tabular}{|c|c|c|c|c|c|c|c|c|}
\hline 0,857 & .051 &, 141 & $-0,015$ & 0,082 & 0,087 & 0,049 & 0,007 & 0,014 \\
\hline 0,854 & 0,154 & 0,096 & 0,132 & $-0,079$ & 0,090 & $-0,052$ & $-0,054$ & $-0,092$ \\
\hline 0,814 & 0,147 & 0,104 & 0,192 & $-0,102$ & $-0,067$ & $-0,056$ & $-0,073$ & $-0,152$ \\
\hline 0,795 & 0,058 & 0,170 & $-0,015$ & 0,066 & $-0,004$ & 0,129 & 0,007 & 0,045 \\
\hline 0,790 & 0,056 & 0,219 & $-0,131$ & 0,166 & 0,101 & $-0,074$ & 0,019 & 0,109 \\
\hline 0,726 & 0,233 & 0,159 & 0,080 & 0,114 & $-0,035$ & $-0,094$ & $-0,051$ & 0,005 \\
\hline 0,574 & $-0,034$ & 0,076 & $-0,015$ & 0,017 & 0,016 & $-0,207$ & 0,450 & 0.051 \\
\hline 0,543 & $-0,040$ & $-0,074$ & -0.077 & 0,091 & 0,506 & 0,184 & 0,118 & 0,082 \\
\hline 0,108 & 0,823 & 0,057 & 0,199 & $-0,018$ & & $-0,034$ & & 0,095 \\
\hline 0,242 & 0,8 & 0,084 & $-0,048$ & 0,074 & $-0,004$ & 0,018 & $-0,051$ & 0,098 \\
\hline 0,0 & 0,7 & $-0,060$ & 0,204 & $-0,039$ & 70 & 0,003 & 0,085 & $-0,145$ \\
\hline 0,109 & 0 & 0,178 & $-0,124$ & 0,346 & $-0,194$ & 0,133 & 0,040 & 0,080 \\
\hline 0,0 & 0,732 & 0,085 & 0,153 & 0,352 & 0,005 & $-0,057$ & -0 & $-0,106$ \\
\hline 0,2 & 0,677 & 0,117 & $-0,234$ & 0,109 & 0,230 & & & 0,088 \\
\hline$-0,071$ & 0,642 & 0,069 & 0,116 & $-0,108$ & 0.095 & & 0.488 & $-0,053$ \\
\hline 0,107 & 0.442 & 0,035 & $-0,018$ & 0,062 & 0.715 & $-0,006$ & & $-0,001$ \\
\hline 0,307 & 0,046 & 0,811 & $-0,020$ & $-0,002$ & $-0,038$ & $-0,034$ & & 0,085 \\
\hline 0,088 & 0 & 07 & -0 . & & -0 & & & 0,154 \\
\hline o, & & 0, & & & -0 , & -0 & -0 & $-0,161$ \\
\hline 0 , & 0 & 0 & & -0 & & & & $-0,088$ \\
\hline 0,131 & 0 & 0,711 & $-0,234$ & 0, & 0.058 & 0 , & 70 & 0,147 \\
\hline 0,043 & & & & & & & & $-0,053$ \\
\hline 0,247 & 6 & 0,576 & 0, & $-0,084$ & & & & $-0,372$ \\
\hline$-0,011$ & $-0,0$ & 0,570 & 0.104 & $-0,320$ & -0 , & 0,080 & 0.444 & 0,060 \\
\hline & & 0.038 & & & & & & 0,208 \\
\hline 0,104 & 2 & $-0,1$ & 0 . & 0.232 & -0 , & -0 & 49 & $-0,110$ \\
\hline$-0,026$ & & & & & & & & 0,009 \\
\hline-0 & & & & & & & & $-0,190$ \\
\hline 0,1 & 0,0 & $-0,017$ & -0, & & 18 & & & 0,374 \\
\hline & & & & & & & & 0,017 \\
\hline$-0,015$ & 0 & 36 & 0,268 & o. & & & 0,255 & $-0,056$ \\
\hline$-0,034$ & 0,0 & $-0,0$ & 0,403 & 0,121 & & 0,090 & 0,657 & $-0,211$ \\
\hline & & & & & & & & 0,032 \\
\hline & $-0,0$ & $-0,0$ & $-0,0$ & & & & & $-0,077$ \\
\hline & & & & & & & & 0,792 \\
\hline $1,1 \%$ & $11,6 \%$ & $8,7 \%$ & $7,0 \%$ & $5,2 \%$ & $4,3 \%$ & $3,8 \%$ & $3,4 \%$ & 3,09 \\
\hline
\end{tabular}

Erklarter Varianzanteil $=68,1 \%$

$7 \%$

bericksichtigte Grenamerte

Tabelle 3: Faktoranalytisch verdichtete Dimensionen der PC-Kompetenz von Unternehmen 
Inhaltlich analog zum ersten setzen sich die Faktoren zwei und drei zusammen. Faktor 2 vereinigt dabei sämtliche abteilungsleiterbezogenen Kennzeichen und wird als "PC-INVOLVEMENT DER ABTEILUNGSLEITER" festgeschrieben. Faktor 3 stellt die entsprechenden Bezugspunkte zur Person des EDV-Beauftragten her und ist deshalb als "PC-INVOLVEMENT DES EDV-BEAUFTRAGTEN" zu interpretieren. ${ }^{171}$

Die Faktoren vier und fünf beziehen sich auf anwenderbezogene Kompetenzkriterien. Anders als bei den vorausgegangenen Größen, differenziert sich hier jedoch das Kompetenzbild.

Die hohen Ladungen der Variablen "kennen sich gut mit PCs aus", "sind stets über neuste PC-Trends informiert" sowie "können den Teilnutzen der einzelnen PC-Komponenten bewerten", deuten auf ein hintergrundspezifisches Technologiewissen hin. Ein derartiges Know how bedingt eine stetige und differenzierte Auseinandersetzung mit PC-bezogene Sachverhalten und hat damit epistemischen Charakter. Dementsprechend wird Faktor 4 als "EPISTEMISCHE ANWENDERKOMPETENZ" bezeichnet.

Faktor 5 weist eine starke heuristische Kompetenzorientierung auf. Hohe Ladungen ergeben sich besonders durch praxisbezogene Variablen "PC-Einsatz im Rahmen der Aufgabenlösung", "arbeiten gerne und regelmäßig mit PCs" und unterstreichen damit den operativen Bezug. Kompetenzeffekte ergeben sich hierbei aufgrund kontinuierlicher Anwendungserfahrungen. Demzufolge läßt sich Faktor 5 als "HEURISTISCHE ANWENDERKOMPETENZ" beschreiben.

Faktor 6 wird hoch geladen von der Variablen "private PC-Nutzung" bei allen vier Personengruppen (Abteilungsleiter, Anwender, Geschätsführer und EDV-Beauftrager). Dieser Faktor manifestiert den Einfluß privater "Home-PC"-Erfahrungen auf das Kompetenzniveau im kommerziellen Einsatz. 172 Neben Unterhaltungs-

171 Die ersten drei Faktoren entsprechen von ihrer Bedeutung in hohem Maße den aufgrund von Indexbildung entstandenen Gruppenkompetenzen in Kap. C.2.2. Dieses unterstreicht zusätzlich noch einmal die inhaltliche Tragfähigkeit der dort zugrunde gelegten Vorgehensweise.

172 Der Markt für Freizeitcomputer wird neben Atari- und Amigasystemen inzwischen weltestgehend durch Personal Computer dominiert. Der lange Zeit stellvertretend für diese Systeme verwendete Begriff "Homecomputer" wandelt sich zunehmend zum "Home-PC". Vgl. zum Stand der Entwicklung von Unterhaltungssystemen: Hink, A., Stiller, H., Consumer Elektronic Show Chicago - Abenteuer Chicago, in: ASM, 7. Jg., Nr. 8, 1992, S. 40. 
software fördert nicht zuletzt auch die parallele Anwendung von Standardanwendungssoftware (Text, Grafik u.a.) im beruflichen und im privaten Umfeld insgesamt das Verständnis des PC-Einsatzes. Die lange Zeit in Frage gestellte Wirkung privater Freizeitanwendungen für eine bessere Anwendungskompetenz im kommerziellen Rahmen kann damit explorativ nachgewiesen werden. Faktor 6 wird als "FREIZEITINDUZIERTER KOMPETENZEFFEKT" definiert.

Der siebte Faktor weist eine starke Verbindung zu den Variablen "PC-Erfahrungen im Unternehmen" und EDV-Erfahrungen im Unternehmen" auf. Beide Merkmale stellen einsatzzeitbedingte Kompetenzeffekte dar und haben damit vornehmlich heuristischen Charakter. Faktor 7 läßt sich als "DATENVERARBEITUNGSERFAHRUNG" eines Unternehmens beschreiben.

Der achte Faktor besitzt im Vergleich zu allen anderen Größen die insgesamt schwächsten Ladungen. Die einzige zunächst akzeptable Verbindung $(0,657)$ besteht zur Variablen "Anwender entwickeln auch eigene Anwendungen". Bei einer differenzierteren Betrachtung der Ladungsstruktur lassen sich jedoch auch bei Abteilungsleitern, Geschäftsführern und dem EDV-Beauftragten noch vertretbare Ladungswerte zu diesem Aspekt feststellen. Obwohl diese drei Größen lediglich zwischen 0,4 und 0,5 liegen, erscheint es sinnvoll diese Variablen in der Interpretation mit zu berücksichtigen. Grund hierfür ist zum einen das sehr niedrige Ladungsniveau aller übrigen Größen und zum anderen der hohe inhaltliche Bezug der Merkmale. Insgesamt läßt sich Faktor 8 unter Berücksichtigung aller vier Einflüsse als "PROGRAMMIERKENNTNIS" bezeichnen.

Faktor 9 steht lediglich mit einer einzigen Variablen in hoher Verbindung. Dies sind die "Stör- und Ausfallerfahrungen", die Unternehmen mit ihren PC-Systemen und diesbezüglichen Kundendienstleistungen bislang gemacht haben. Angesichts des direkten Zusammenhangs wird Faktor 9 entsprechend als "STÖRUND AUSFALLERFAHRUNG" bezeichnet.

Die Analyseergebnisse haben zusammenfassend gezeigt, daß eine Operationalisierung der beiden Bedürfnisdimensionen "Abhängigkeitsgrad" und "Kundenkompetenz" mit Hilfe einer explorativen Faktorenanalyse erfolgreich durchgeführt werden kann. Damit kann Hypothese $\mathbf{H}_{\mathbf{S}} \mathbf{1}$ als bestätigt angesehen werden. Die extrahierten 6 bzw. 9 Faktoren bilden den Ausgangspunkt der weiteren Analyse zur Bildung kundendienstbezogener Bedürfnissegmente. 

Computer

\subsection{1}

Methodische Grundlagen zur Gruppenbildung

Die Bildung bedürfnisbezogener Kundendienstsegmente steht im Mittelpunkt der folgenden Betrachtung. Die hierzu aufgestellte Untersuchungshypothese lautet:

HS2: Im Markt für kommerzielle Personal Computer lassen sich trennscharfe Segmente mit jeweils homogenen Servicebedürfnissen identifizieren und beschreiben. Unterschiedliche Reaktionen auf Marketingimpulse von Kundendienstanbietern kennzeichnen dabei das Bild in den einzelnen Teilmärkten.

Die Aufteilung des Gesamtmarktes erfolgt mit Hilfe der Clusteranalyse. ${ }^{173}$ Zielsetzung dieses Verfahrens ist, eine Anzahl von Unternehmen (Objekte), entsprechend der Ähnlichkeit ihrer bedürfnisbezogenen Kundendienstvorstellungen, in eine natürliche Ordnung von sich unterscheidenden Gruppen oder Klassen zu bringen. Als clusterbildende Variablen werden die extrahierten Faktoren der beiden Brückenkonstrukte Abhängigkeitsgrad und Kundenkompetenz herangezogen. Sie sind in Tabelle 4 zusammenfassend dargestellt.

Zur Durchführung von Clusteranalysen stehen eine Reihe unterschiedlicher Algorithmen zur Verfügung. Gemäß der Vorgehensweise im Filterungsprozeß lassen sich insbesondere das hierarchische und das iterative (partitionierende) Verfahren nennen. 174 In der vorliegenden Untersuchung wird mit dem Verfahren von Ward ein partitionierender Ansatz gewählt. 175 Der Rechenalgorithmus be-

$173 \mathrm{Zu}$ einer ausführlichen Darstellung der Clusteranalyse vgl. Meffert, H., Marketingforschung und Käuferverhalten, a.a.O., S. 267 ff.; Backhaus, K., et al., Multivariate Analysemethoden, a.a.O., S. 115 ff.; Bleymüller, J., et al., Multivariate Analyse für Wirtschaftswissenschaftler, Münster 1983, S. 125 ff.; Steinhausen, D., Langer, K., Clusteranalyse, Berlin, New York 1977; Vogel, F., Probleme und Verfahren der numerischen Klassifikation, Göttingen 1975, S. 35 ff.

174 Während bei hierarchischen Verfahren eine sukzessive Aufteilung der untersuchten Objekte vollzogen wird, werden die Objekte bei iterativen Ansätzen simultan (um)gruppiert. Vgl. zur systematischen Darstellung und Diskussion verschiedener Gruppierungsalgorithmen der Clusteranalyse Backhaus, K., et al., Multivariate Analysemethoden, a.a.O., S. 133 f.

175 Als Āhnlichkeitsmaß wurde dem Verfahren die quadrierte euklidische Distanz zugrunde gelegt. 
ruht dabei auf einer Verbesserung des Varianzkriteriums durch eine iterative Veränderung einzelner Cluster. 176

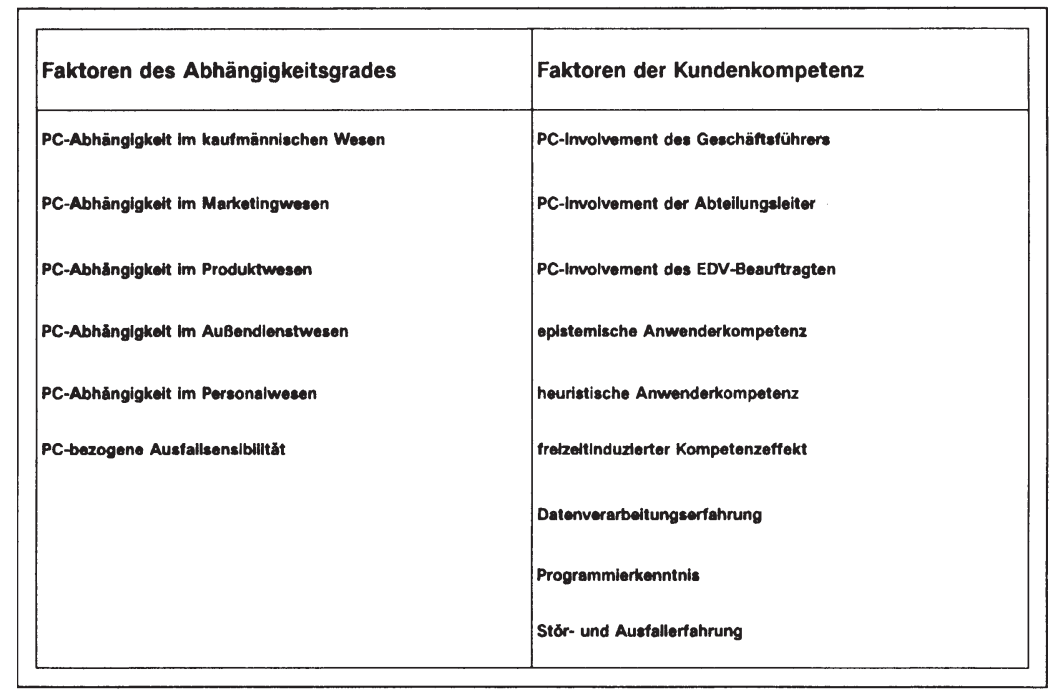

Tabelle 4: Input-Faktoren der kundendienstbezogenen Clusteranalyse

Ziel des Ward-Verfahrens ist es, diejenigen Objekte zu vereinigen, die ein definiertes Heterogenitätsmaß am geringsten vergrößern, d.h. daß sich die Streuung in einzelnen Gruppen möglichst wenig erhöht. Damit wird dem zentralen Segmentierungsziel - Bildung homogener Gruppen - a priori in besonderer Weise entsprochen. Das herangezogene Varianzkriterium wird als Fehlerquadratsumme (FQS) bezeichnet.

Die Bestimmung der optimalen Clusteranzahl kann mit Hilfe des "Elbow-Kriteriums" erfolgen. Dabei wird der Entwicklungsverlauf der Fehlerquadratsumme betrachtet indem man die FQS gegen die entsprechende Clusterzahl in einem Koordinatensystem abträgt (vgl. Abbildung 21, obere Teilabbildung). Mit steigender Gruppenzahl sinkt das Heterogenitätsmaß der Lösung. Die Auswahl der

176 Bergs kommt in seinen Untersuchungen zu dem Ergebnis, daß das Ward-Verfahren zu sehr guten Gruppierungsergebnissen führt und die einzelnen Objekte den Gruppen i.d.R. richtig zuordnet. Vgl. Bergs, S., Optimalität bei Cluster-Analysen, Münster 1981, S. 91 f. 
"richtigen" Clusteranzahl erfolgt an der Stelle, an der ein weiterer Gruppenzusammenschluß einen beträchtlichen Zuwachs der FQS zur Folge hat bzw. eine größere Anzahl Gruppen nur zu einer geringeren Verbesserung des Varianzkriteriums führt.

In der Praxis ist ein solch deutlicher Elbow häufig jedoch nicht erkennbar. Statt dessen fält die Kurve scheinbar völlig gleichmäßig ab.177 Diese Situation besteht auch im vorliegenden Fall. Analog zur absoluten Darstellung der Fehlerquadratsumme läßt sich jedoch auch deren prozentualer Zuwachs abbilden (Abbildung 21, untere Teilabbildung). 178 Diese Alternative bietet sich gerade bei relativ geringen Zuwachsveränderungen an.

Neben dem Elbow-Kriterium ist eine Clusterlösung vor allem aber auch vor dem Hintergrund inhaltlich sinnvoller Interpretationsmöglichkeiten zu bewerten und auszuwählen. Dieser Aspekt ist umso stärker zu berücksichtigen, je weniger aussagekräftig und eindeutig analytische Auswahlkriterien sind.

Aufgrund der geschilderten Zusammenhänge und unter besonderer Berücksichtigung inhaltlicher Gesichtspunkte wird im folgenden eine 6-Cluster-Lösung zugrunde gelegt.

In einem ersten Schritt erfolgt die inhaltliche Darstellung der einzelnen Segmente auf der Grundlage von Mittelwertunterschieden. 179 Dies geschieht zunāchst mit Hilfe clusterbildender Faktoren. Dieses Bild wird um makro- und mikroanalytischer Gesichtspunkte ergänzt. Hierzu werden unternehmensbezogene, verhaltensbezogene und hauptleistungsbezogene Kriterien sowie ausgewählte Kennzeichen des EDV-Beauftragten betrachtet. Die dritte hinzugezogene Variablen

177 Vgl. Bleymüller, J., et al., Multivariate Analyse für Wirtschaftswissenschaftler, a.a.O., S. 174.

178 Der prozentuale Zuwachs der FQS läßt sich mit Hilfe der Formel $\frac{\text { FQS }_{n}-F_{n-1}}{F Q S_{n-1}}{ }_{1} 100$ berechnen: Beim Übergang von einer 5- zu einer 6-Gruppenlösung ist dieser Effekt in der vorliegenden Situation stärker, als beim Übergang von einer 6- zu einer 7-Gruppenlösung.

179 SPSS bletet mehrere Alternativen zur Mittelwertuntersuchung von Gruppen an. Zum einen können diese Unterschlede im Rahmen einer Diskriminanzanalyse berechnet werden. Zum anderen ist dies mit Hilfe der Prozedur MEANS möglich. Neben der gruppenbezogenen Mittelwertausgabe bietet dieses Verfahren auch noch die Möglichkeit einer Signifikanzprüfung. 


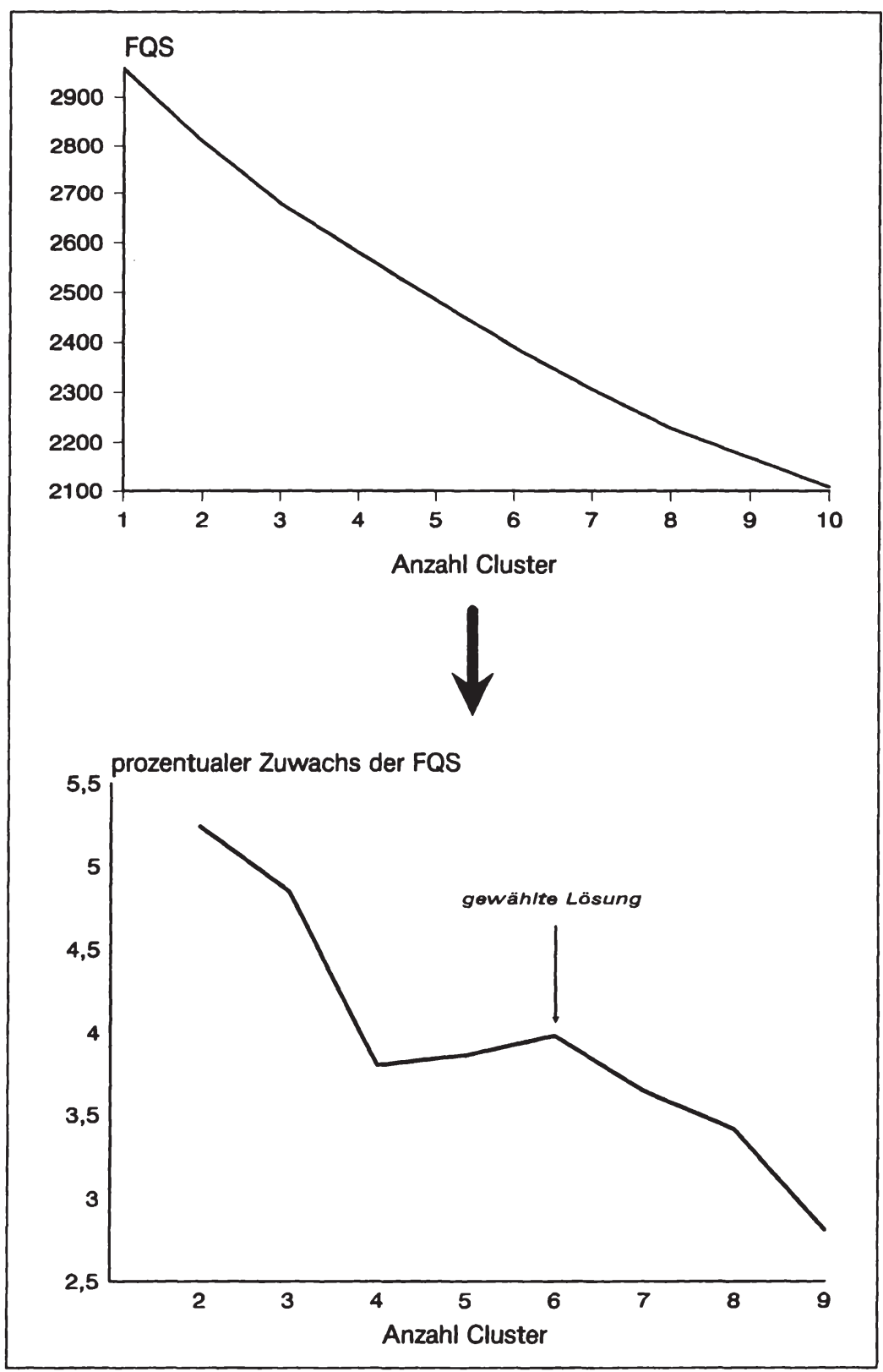

Abbildung 21: Varianzkriterium für die Clusterlösung 
gruppe besteht aus reaktionsbezogenen Kriterien. Treffen die Überlegungen der bedürfnisorientierten Segmentabgrenzung mit Hilfe abhängigkeitsgrad- und kundenkompetenzbezogener Faktoren zu, dann müssen sich die einzelnen Gruppen auch hinsichtlich ihrer produkt-, kommunikations-, distributions- sowie kontrahierungsbezogenen Anforderungen und Erwartungen unterscheiden.

In einem zweiten Schritt ist schließlich die Trennkraft und damit die Güte des entwickelten Segmentierungsergebnisses zu überprüfen. Die Analyse der Trennschärfe zwischen den gefundenen Gruppen kann mit Hilfe einer Diskriminanzanalyse durchgeführt werden. Hierbei werden die identifizierten Cluster als Gruppen vorgegeben und die Bedürfnisfaktoren als unabhängige Variablen betrachtet. 180

\subsection{Darstellung bedürfnisbezogener Kundendienstsegmente}

Das Clusterergebnis führt zu den in Abbildung 22 dargestellten Gruppengrößen. Die Übersicht dokumentiert zum einen eine befriedigende MindestgruppengröBe. Zum anderen besteht, trotz der relativ stärkeren Besetzung des Clusters 1, ein insgesamt ausgewogenes Verhältnis. Alle gefundenen sechs Segmente weisen eine jeweils servicetypische Struktur auf, die im folgenden erläutert wird. ${ }^{181}$

$180 \mathrm{Vgl}$. zu dieser Vorgehensweise Backhaus, K., et al., Multivariate Analysemethoden, a.a.O., S. 151.; Heinemann, G., Betriebstypenprofilierung und Erlebnishandel, Wiesbaden 1989, S. 82 ff.

$181 \mathrm{Vgl}$. hierzu auch die Mittelwertabweichungen der Faktoren und Ausgangsvariablen, die in den Tabellen Al-2 bis Al-6 im Anhang dieser Arbelt dargestellt sind. 


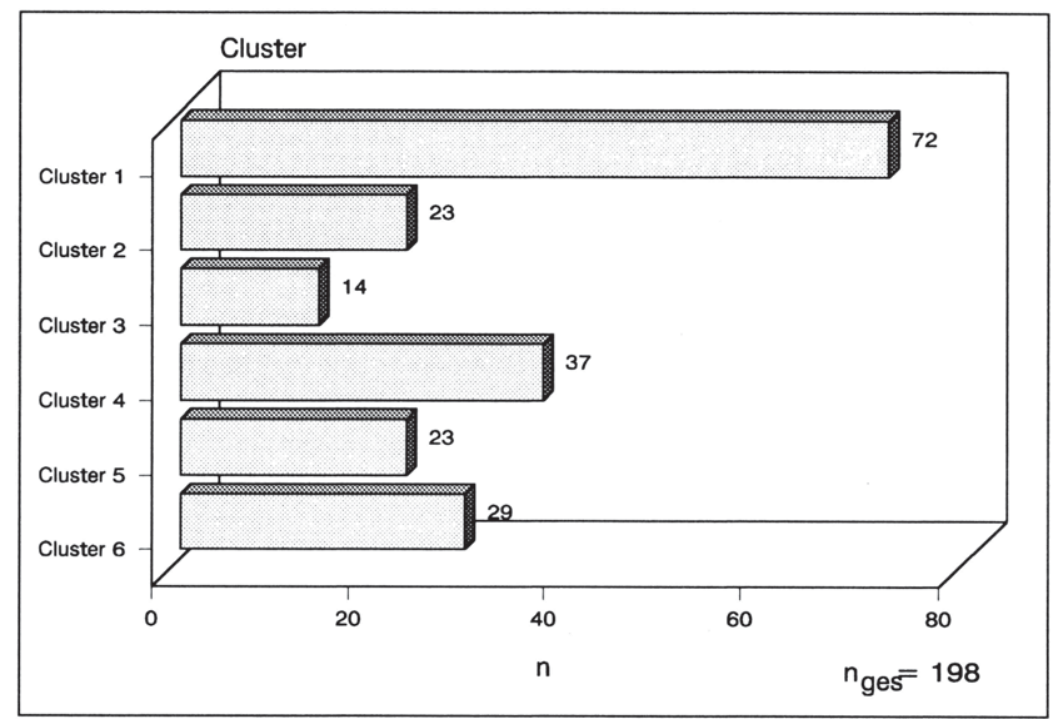

Abbildung 22: Besetzung der 6-Cluster-Lösung

\subsection{Das kundendienstunsensible Segment}

\section{Bedürnisbestimmende Faktoren:}

Das erste Segment $(n=72)$ ist gekennzeichnet durch eine insgesamt durchschnittliche PC-Integration im Unternehmen. Unterschiede bestehen lediglich in einer geringfügig stärkeren Nutzung im Personalbereich und einer schwächeren Bedeutung im Produktwesen. Die Ausfallsensibilität liegt ebenfalls in der mittleren Norm. Die bisherigen Stör- und Ausfallerfahrungen mit Personal Computern sind im Vergleich durchschnittlich. Angesichts der insgesamt hohen Bewertung dieser Variablen (kaum Ausfälle), ist dieses Cluster eher wenig von Negativereignissen betroffen worden.

Die Kompetenzsituation der Unternehmen ist in der Breite durchschnittlich und wird dominiert durch die EDV-Beauftragten. Auffällig ist zudem, die rein heuristische Kompetenz der Anwender. Eine epistemische Kompetenz besteht nicht. Spezial-Know how, wie z.B. Programmierkenntnisse steht nicht zur Verfügung. Der gesamte DV-Erfahrungszeitraum der Unternehmen ist verhältnismäßig kurz. 
Makro- und mikroanalytische Kriterien:

Ein vergleichsweise hoher Anteil der Unternehmen kommt aus dem Dienstleistungsbereich. Industriebetriebe sind dagegen kaum vertreten. Sowohl der Stellenwert von Personal Computern im Rahmen der gesamten Datenverarbeitung, als auch ihre Bedeutung für den Unternehmenserfolg wird durchschnittlich beurteilt. Strategischen Charakter weisen diese Systeme im Unternehmensprozeß damit offenbar nicht auf. Dies erscheint umso verwunderlicher, da nur etwas mehr als die Hälfte aller Unternehmen (54\%) zusätzliche Computersysteme einsetzen. Die PC-Konfigurationen weisen kein charakteristisches Erscheinungsbild auf. Dies betrifft hardware-, aber auch softwarebezogene Aspekte.

Im Rahmen des Hauptleistungskaufs vereinbart dieses Segment zum überwiegendem Teil Schulungsleistungen und schließt Servicevertäge (Hardware und Software) ab. Auf besondere Gewährleistungsvereinbarungen wird weniger Wert belegt.

Die EDV-Beauftragten dieses Clusters besitzen eine vergleichsweise kurze EDVErfahrung. Ein Drittel besitzt eine aufgabenspezifische Ausbildung. Dies liegt im Mittel der Gesamtbetrachtung. Eine zusätzliche Aufgabenvorbereitung und Weiterbildung findet in durchschnittlichem Umfang statt. Zentrales Instrument ist hierfür das "learning by doing"-Prinzip. Fachseminare werden verhältnismäßig selten besucht. Das Know how dieser Fachvertreter ist damit im wesentlichen erfahrungsbestimmt zu beurteilen. $53 \%$ benutzen auch im privaten Umfeld einen Personal Computer.

\section{Reaktionsbezogene Kriterien:}

Im Hinblick auf kommunikationspolitische Gesichtspunkte ist zunächst ein recht undifferenziertes Einstellungsmuster gegenüber Kundendienstleistungen festzustellen. Das Cluster sieht weder besonders zwingende Servicemotive, noch bestehen entsprechende Barrieren und Vorbehalte. Dementsprechend durchschnittlich ausgeprägt ist auch der Bekanntheitsgrad verschiedener Kundendienste. Dies ist jedoch vor dem Hintergrund geringer Serviceerfahrungen zu sehen. Dieser Aspekt korrespondiert inhaltlich mit der bislang geringen Ausfallbetroffenheit. Das Informationsverhalten zu PC- und kundendienstbezogenen Themen ist durchschnittlich, z.T. sogar schwächer zu beurteilen. Besonders wenig genutzt werden Fachzeitschriften und Anbieterinformationen. Auch ein Austausch mit Kollegen im Unternehmen findet kaum statt. 
In bezug auf das produktpolitische Kundendienstangebot weist Cluster 1 ein relativ ausgewogenes Erscheinungsbild auf. Die Bedeutung, die PC-bezogenen Serviceleistungen beigemessen wird, deckt sich im wesentlichen mit den Erfahrungen (Zufriedenheit), die bislang mit der Inanspruchnahme derartiger Dienste erzielt wurden (vgl. Abbildung 23).

Nennenswerte Divergenzen treten dabei lediglich bei den Serviceleistungen Installation/Inbetriebnahme, Hot Line Service und Wartung/Reparatur vor Ort auf. Die Beurteilung zeigt, daß die Erwartungen in diesen Bereichen höher lagen als die Zufriedenheit mit den Kundendienstresultaten. Demgegenüber wurden im Bereich Netzwerkeinrichtung die Erwartungen deutlich übertroffen.

Besondere distributions- und kontrahierungspolitische Anforderungen und Leistungsvorstellungen an den Kundendienstleister bestehen nicht. Die Erwartungen sind diesbezüglich insgesamt durchschnittlich ausgeprägt.

\section{Zusammenfassung:}

Personal Computer besitzen in Cluster 1 keinen strategischen Stellenwert. Der geringe Einsatz anderer EDV-Systeme verstärkt den Eindruck, daß der elektronischen Datenverarbeitung in diesen Unternehmen insgesamt eine geringe Bedeutung beigemessen wird.

Mangelnde Betroffenheit aufgrund bislang fehlender Negativerfahrungen ebenso, wie ein grundsätzlich fehlendes Bewußtsein für die mögliche Risiken eines PC-Einsatzes, führen dazu, daß Sinn und Nutzen von Kundendienstleistungen nicht reflektiert werden. Aufgrund des eher introvertiert zu kennzeichnenden Informationsverhaltens zeigen Aufklärungsbemühungen von Serviceanbietern diesbezüglich offenbar wenig Wirkung.

Die mangelnde Problematisierung des innerbetrieblichen DV-Einsatzes bedingt in der Konsequenz die unzureichende Auseinandersetzung mit kundendienstpolitischen Fragen. Mitglieder dieses Segments sind für servicebezogene Aspekte bislang zu wenig sensibilisiert. 


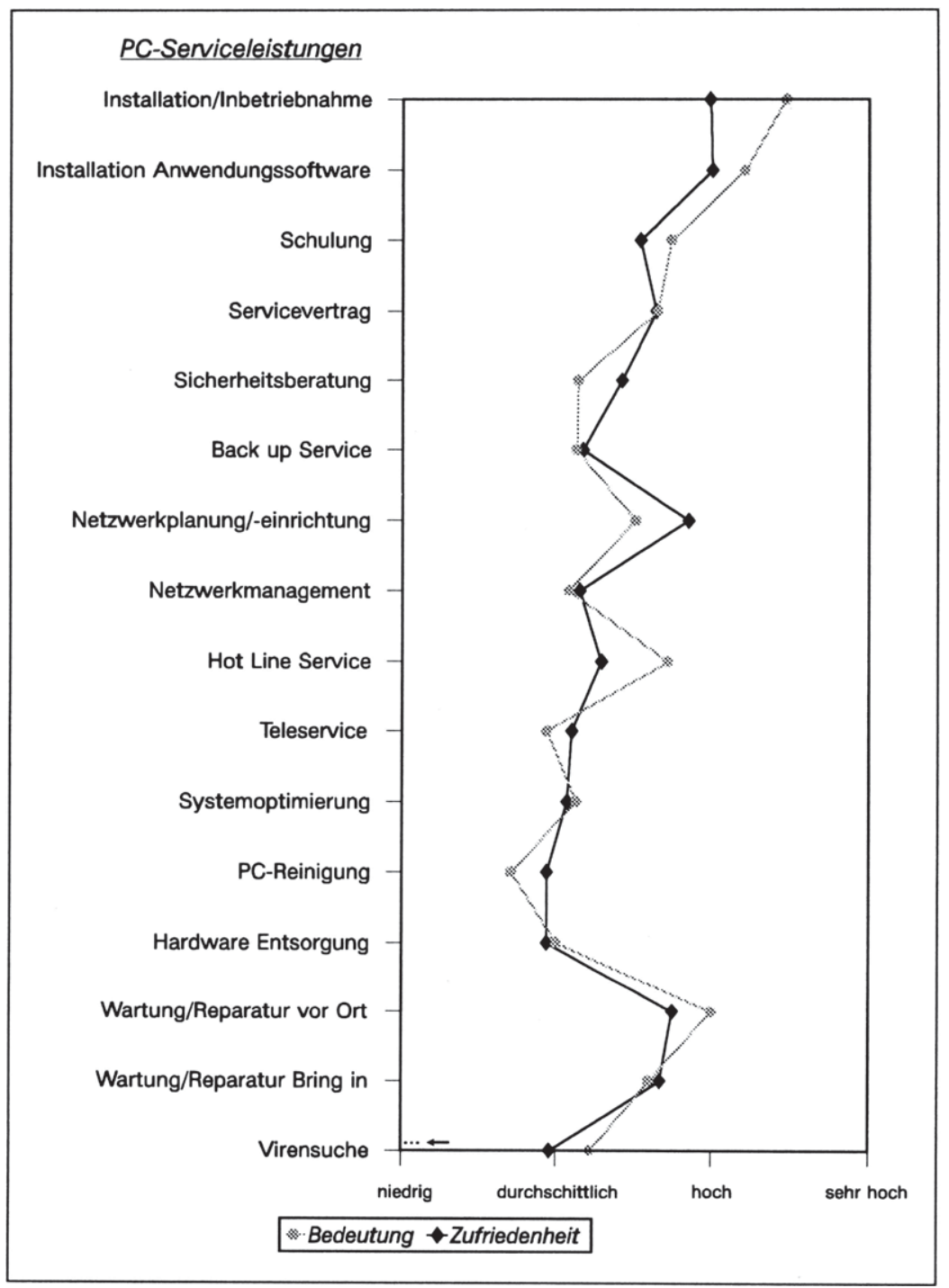

Abbildung 23: Kundendienstbezogene Bedeutungs- und Zưriedenheitsbeurteilung des kundendienstunsensiblen Segments 


\subsection{Das Kundendienst-Nischensegment}

\section{Bedürfnisbestimmende Faktoren:}

Das zweite Segment $(n=23)$ weist eine ganz spezifische PC-Implementierung auf. Während in allen anderen Unternehmensbereichen eine unterdurchschnittliche Abhängigkeit besteht, liegt im Außendienstwesen eine außerordentliche starke PC-Verknüpfung vor. Die Ausfallsensibilität der Systeme ist gering ausgeprägt. Ursache hierfür mag sein, daß im Fall von Systemdefekten bei mobilen Systemen (Laptops), häufig unmittelbar auf Ersatzrechner zurückgegriffen werden kann. Damit ist die Aufgabenerfüllung während der Reparaturphase nicht unterbrochen. Die bisherigen Stör- und Ausfallerfahrungen dieses Clusters liegen im durchschnittlichen Rahmen.

Die Kompetenzsituation im Nischensegment zeigt sich insgesamt durchschnittlich. Ein überdurchschnittliches PC-Involvement besteht bei Geschäftsführern. Spezifische Programmierkenntnisse bestehen nicht. Die Unternehmen besitzen eine relativ lange EDV-Erfahrung.

Makro- und mikroanalytische Kriterien:

Segment 2 weist tendenziell ein eher dienstleistungsorientiertes Profil auf. Viele Versicherungen gehören zu dieser Gruppe. Handelsunternehmen sind weniger vertreten. Sowohl die Bedeutung der PCs für den Unternehmenserfolg, als auch inr Stellenwert im Rahmen der gesamten Unternehmens-EDV wird als überdurchschnittlich hoch eingestuft. Der Mitarbeiteranteil, der in den Unternehmen mit PCs arbeitet ist mit $22 \%$ vergleichsweise hoch. $70 \%$ der Segmentmitglieder setzen neben PCs zusätzliche Computersysteme ein. In erster Linie handelt es sich hierbei um Großrechneranlagen. Innerhalb der Gesamt-EDV besitzen PCs damit als Außendienstlösung einen ganz spezifischen und strategischen Stellenwert.

Der Anteil an mobilen PCs ist sehr hoch. Der Softwareeinsatz ist in erster Linie durch Standardanwendungssoftware gekennzeichnet. Darüber hinaus werden zusätzlich auch Individualsoftwarelösungen eingesetzt.

Grundservices werden nur in sehr geringem Umfang genutzt. Die Unternehmen beschränken sich i.d.R. auf reine Hard- und Softwarekäufe.

Die aufgabenbezogene Erfahrung der EDV-Beauftragten ist relativ groß. 39\% besitzen eine fachspezifische Berufsausbildung. Dieser Anteil ist relativ hoch und 
liegt im Vergleich zu anderen Segmenten an zweiter Stelle. Die Vorbereitung und Weiterbildung wird sehr differenziert vollzogen. Während Herstellerschulungen nur in geringem Umfang genutzt werden, stellen herstellerunabhängige Kurse und ganz besonders spezielle Fachseminare die am häufigsten in Anspruch genommenen Angebote dar. 57\% der EDV-Beauftragten benutzen auch privat einen PC.

\section{Reaktionsbezogene Kennzeichen:}

In bezug auf kommunikationspolitische Aspekte ist festzustellen, daß Kundendienst besonders unter dem Gesichtspunkt einer höheren Systemverfügbarkeit befürwortet wird. Demgegenüber ist Service nach der vorherrschenden Auffassung nur wenig dazu geeignet, PC-Investitionen abzusichern. Weitere Servicebarrieren sind in diesem Segment nicht festzustellen. Daterıschutzängste und sonstige Bedenken bestehen nicht. Die bisherigen Kundendiensterfahrungen sind positiv. Die allgemeine Bekanntheit des kundendienstpolitischen Leistungsspektrums ist jedoch vergleichsweise gering. Dies ist bemerkenswert, da das Informationsverhalten der EDV-Beauftragten ausgesprochen breit und intensiv angelegt ist. Neben Zeitschriften und Literatur werden verschiedenste Anbieterund Kollegenkontakte, Tagungen sowie Schulungen als Informationsquelle genutzt.

Dem Kundendienst wird insgesamt eine durchschnittliche Bedeutung beigemessen. Die derzeit bestehenden Standards des Service werden positiv angenommen. Unzufriedenheiten an der Leistungserbringung bestehen dagegen bei Hot Line Diensten und in Bereichen des Wartungs- und Reparaturservice (vgl. Abbildung 24). Neben einer Qualitätsverbesserung bei diesen Leistungen werden im Rahmen der produktpolitischen Vorstellungen besondere Anforderungen in bezug auf ein umfangreiches Serviceangebot, die Einhaltung zugesagter Termine und kurze Reaktionszeiten gestellt.

Hinsichtlich distributionspolitischer Erwartungen legt das Kundendienst-Nischensegment weniger Wert auf Fernbetreuungsmöglichkeiten (Teleservice). Grund hierfür ist nicht zuletzt der hohe Anteil mobiler Systeme.

In bezug auf kontrahierungsbezogene Aspekte wird vor allem Wert auf eine bedarfsgerechte Ausgestaltung der Servicekonditionen gelegt. Hardwarepreise stehen dagegen weniger im Mittelpunkt der Überlegungen. 


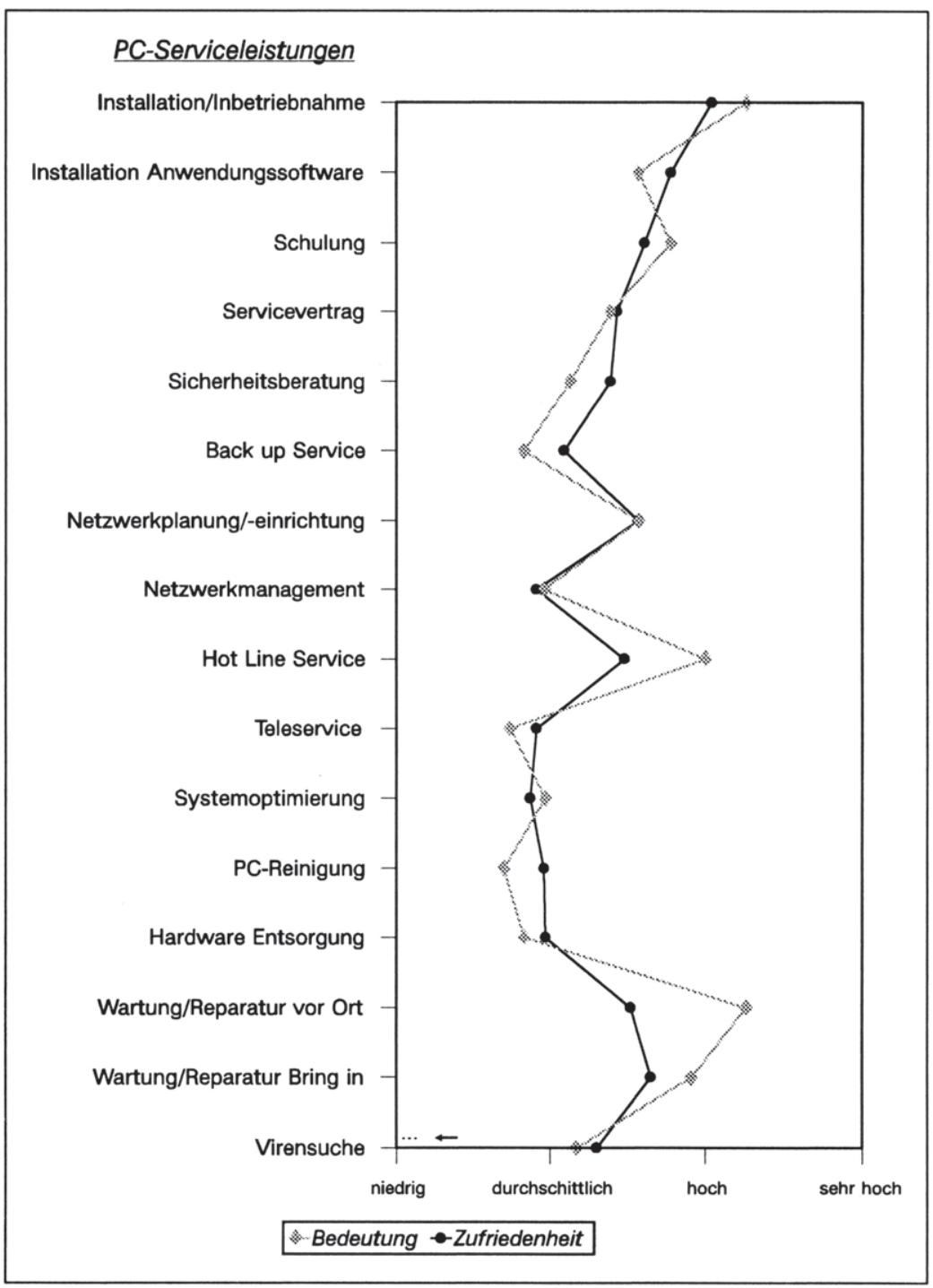

Abbildung 24: Kundendienstbezogene Bedeutungs- und Zufriedenheitsbeurteilung des Kundendienst-Nischensegments 


\section{Zusammenfassung:}

Cluster 2 ist ein Nischensegment, in dem Personal Computer in strategisch bedeutender, jedoch sehr spezieller Funktion eingesetzt werden. Die Relevanz dieser Technologie dokumentiert sich u.a. auch durch das hohe Involvement der Unternehmensleitung. Kundendienst wird in seiner Bedeutung akzeptiert und adaptiert. Dabei ist jedoch festzustellen, daß eine unbefriedigende Serviceproduktbekanntheit eine höhere Potentialausschöpfung bislang behindert.

\subsection{Das Standard-Kundendienstsegment}

\section{Bedürfnisbestimmende Faktoren:}

Im dritten Segment $(n=14)$ besteht in allen Unternehmensbereichen eine sehr hohe PC-Abhängigkeit. Im Marketing und im Produktwesen ist diese Situation besonders stark ausgeprägt. Eine Ausnahme bildet lediglich das Außendienstwesen. Hier besteht eine durchschnittliche PC-Integration. Dementsprechend hoch kennzeichnet sich auch die Ausfallsensibilität der Systeme. Negative Erfahrungen mit Stör- und Ausfallsituationen bei PCs existieren bislang noch nicht bzw. in nur ganz geringem Maße.

Die in der Breite durchschnittliche PC-Kompetenz wird dominiert durch ein sehr hohes Involvement der Geschäftsleitung und des EDV-Beauftragten. Zudem ist die Kundenkompetenz durch sehr gute Programmierkenntnisse sowie eine lange DV-Tradition gekennzeichnet.

\section{Makro- und mikroanalytische Kriterien:}

Die Unternehmen des Segments sind in erster Linie Industriebetriebe. Dienstleister gehören nicht zu dieser Gruppe. Obwohl 70\% aller Gruppenmitglieder zusätzlich auch noch andere Computersysteme einsetzen, ist die Bedeutung von Personal Computer für den Unternehmenserfolg, ebenso wie deren Stellenwert im Rahmen der gesamten EDV-Lōsung sehr hoch. Diese Systeme stellen damit eine wesentliche strategische Komponente der Datenverarbeitung in Cluster 3 dar.

Das Segment setzt in überdurchschnittlichem Umfang leistungsstarke Systeme (386er/486er) ein. Im Rahmen der durchschnittlich stark genutzten, jedoch sehr breit gefächerten Anwendungssoftwarepalette werden Datenbankprogramme 
vergleichsweise intensiv eingesetzt. Neben Standardsoftware sind vielfach auch intern selbst erstellte Individualsoftwarelösungen vorhanden.

Das Standardsegment ist ebenso wie Cluster 2 grundserviceabgeneigt. Mit Ausnahme besonderer Gewährleistungsvereinbarungen, beschränken sich Unternehmen beim Systemkauf auf Hard- und Softwarekomponenten.

Die EDV-Beauftragten besitzen im Verhältnis zu den anderen Segmenten die längste Erfahrung. Im Gegensatz dazu haben jedoch nur $21 \%$ eine fachspezifische Berufsausbildung durchlaufen. Dieses scheinbare Manko wird durch ein intensives Aufgabenvorbereitungs- und Weiterbildungsverhalten kompensiert. Besonders intensiv werden dabei ausgewählte Fachseminare besucht. Zusätzliche Kompetenzeffekte ergeben sich durch Kollegenkontakte sowie durch "learning by doing". $57 \%$ verwenden auch privat PCs.

\section{Reaktionsbezogene Kennzeichen:}

Die kundendienstpolitische Einstellung wird in hohem Maße durch Kundendienstmotive bestimmt. Unternehmen dieses Clusters artikulieren ihren Servicebedarf und sind explizit davon überzeugt, daß elektronische Datenverarbeitung, trotz moderner Hardware, ohne entsprechende Kundendienstunterstützung nicht möglich ist. Service erhöht die Systemverfügbarkeit und ermöglicht eine präzisere Kalkulation der laufenden EDV-Kosten. Entsprechende Personalkapazitäten können unternehmensintern nicht aufgebaut werden. Positive Kundendiensterfahrungen bei anderen EDV-Systemen fördern diese Haltung. Einzig gravierender Nachteil ist die z.T. schwierige und umständliche Kontaktaufnahme zum Kundendienstanbieter.

Diese außerordentlich positive Einstellung ist auch vor dem Hintergrund eines sehr intensiven Informationsverhaltens zu sehen. Vergleichbar dem Kundendienst-Nischensegment werden sämtliche Instrumente zur Informationsbeschaffung herangezogen. Hierzu zählen Fachzeitungen und -literatur, Herstellerinformationen, Kontakte zu Anbietern und Kollegen, Schulungen und Tagungen.

Insgesamt betrachtet wird sowohl die Bedeutung von Kundendiensten als auch die Servicezufriedenheit durchschnittlich bewertet. Eine differenziertere Betrachtung offenbart jedoch, daß bei einer Reihe von Kundendienstprodukten die Qualität der Leistungserbringung der beigemessenen Bedeutung nicht gerecht werden konnte (vgl. Abbildung 25). 


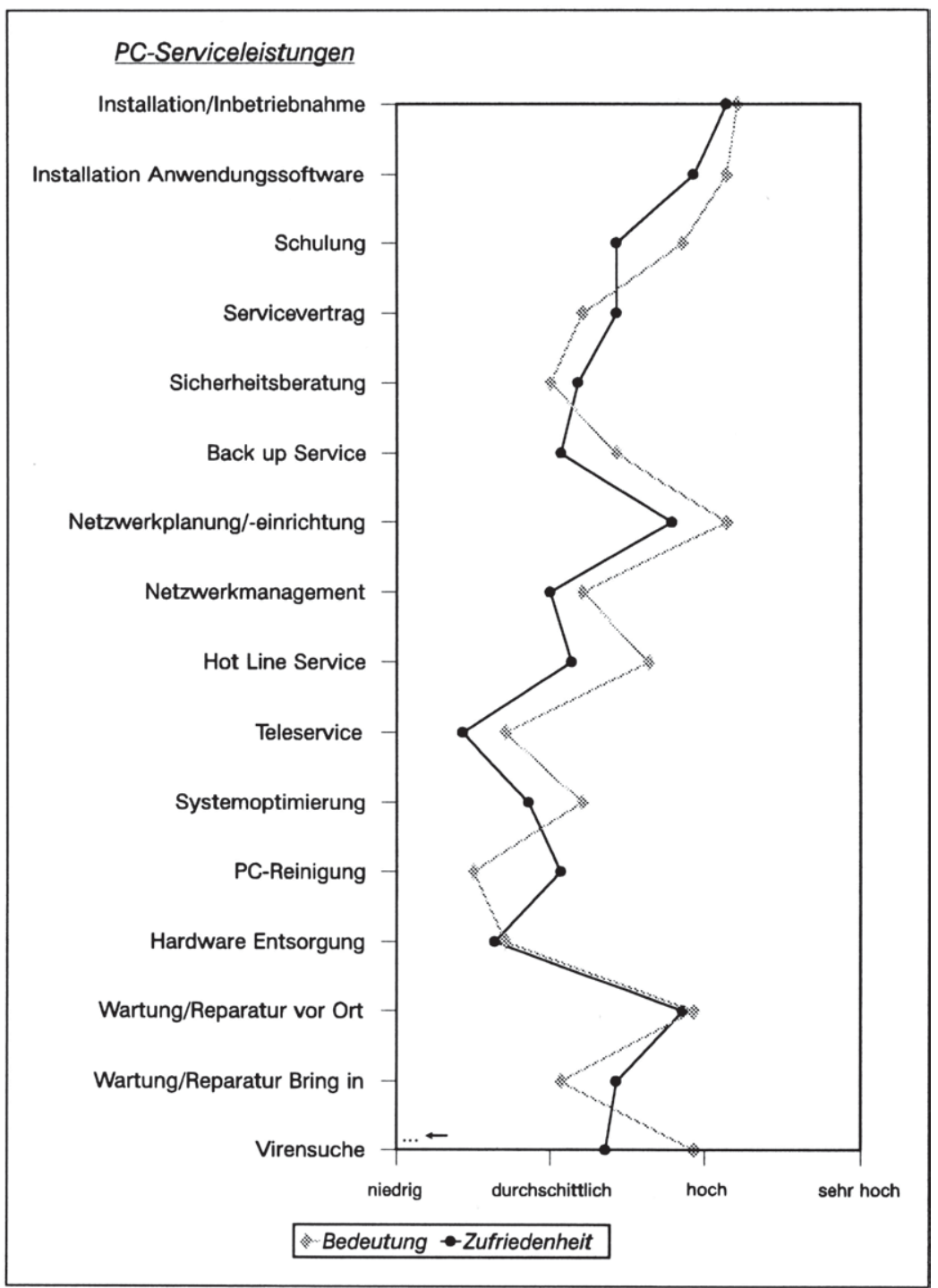

Abbildung 25: Kundendienstbezogene Bedeutungs- und Zutriedenheitsbeurteilung des Standard-Kundendienstsegments 
Dies trifft u.a. besonders auf Schulungen, Netzwerkdienste, Hot Line- und Teleservices, Systemoptimierungen und die Virensuche zu. Dagegen werden bei Serviceverträgen und Bring-in Diensten die Erwartungen übertroffen.

Zu den zentralen produktpolitischen Forderungen gehören im Standard-Kundendienstsegment ferner ein umfangreiches Serviceangebot, eine effiziente Hot Line Unterstützung sowie ein adäquates Technikerauftreten.

Angesicht der hohen Ausfallsensibilität legen die Unternehmen in bezug auf distributionsbezogene Gesichtspunkte besonders hohen Wert auf eine 24-stündige Kundendiensterreichbarkeit, eine 24-stündige Technikerverfügbarkeit, sowie innovative Kundendienstkanäle (Hot Line, Teleservice).

Servicepreise stellen bislang kein zentrales Argument in dieser Gruppe dar. Wesentlich bewußter reagieren die Unternehmen jedoch in bezug auf Hardwarepreise. Überzogene Forderungen führen hier u.U. zum Anbieterwechsel.

\section{Zusammenfassung:}

Cluster 3 ist ein in hohem Maße kundendienstgeneigtes Segment mit einer komplexen, strategisch implementierten PC-Einbindung. Der Nutzen von Serviceleistungen wird offenbar ohne nennenswerte Einwände verstanden. Kundendienst wird als notwendiger Garant eines funktionierenden und störungsfreien PC-Betriebs gesehen. Der Bedarf artikuliert sich dabei von durchaus kompetenter Nachfragerseite (EDV-Beauftragte, Geschäftsführer).

Die gegenwärtig bestehenden Leistungsstandards decken die Bedürfnissituation dieser Unternehmen ab (Standard-Kundendienst). Defizite bestehen allenfalls in einer z.T. nicht zufriedenstellenden Serviceproduktqualität, die bislang von Nachfragerseite jedoch noch nicht entsprechend moniert wird.

\subsection{Das kundendienstskeptische Segment}

\section{Bedürfnisbestimmende Faktoren:}

Cluster 4 ( $n=37)$ weist insgesamt gesehen eine geringe PC-Abhängigkeit auf. Während Personal Computer im Personal-, Marketing- und Außendienstwesen noch durchschnittlich integriert sind, ist eine Einbindung im kaufmännischen Bereich nicht bzw. kaum gegeben. Eine geringfügig höhere Relevanz besteht da- 
gegen im Produktwesen. Die Ausfallsensibilität wird durchschnittlich beurteilt. In bezug auf Störungen und Ausfälle hat Segment 4 bislang die schlechtesten Erfahrungen aller Gruppen gemacht.

Die Kompetenzsituation in Cluster 4 stellt sich sehr differenziert dar. Das PC-Involvement der Unternehmens- und Bereichsleitung ist sehr gering. Auch eine heuristische Anwenderkompetenz ist nur unterdurchschnittlich gegeben. Starke Kompetenzeffekte bestehen im Unternehmen jedoch seitens der Anwender bezüglich ihrer epistemischen PC-Kompetenz. Dieses Know how wird zudem sehr stark durch Freizeitnutzungseffekte gefördert.

\section{Makro- und mikroanalytische Kriterien:}

Segment vier umfaßt vorwiegend Handelsunternehmen und zu geringerem Teil auch Industriebetriebe. $84 \%$ der Organisationen setzen neben PCs zusätzliche Computer ein. Dies sind in erster Linie Mainframe-Computer, aber auch mittlere Systeme sind installiert. Der Stellenwert, den PCs im Rahmen der gesamten EDV-Lösung besitzen, ist äußerst gering. Ebenso geringwertig wird die Bedeutung dieser Rechner für den Unternehmenserfolg eingestuft. Der installierte PCBestand besteht zudem vornehmlich aus leistungsschwächeren Geräten (XTs). Personal Computer besitzen folglich als Arbeitsmittel allenfalls operativen Charakter.

Grundservices werden im Rahmen des Hauptleistungskaufs nicht berücksichtigt. Ebenso wie Cluster 2 und 3 finden hierbei ausschließlich hard- und softwarebezogene Gesichtspunkte Beachtung. Eine Ausnahme bilden besondere Gewährleistungsvereinbarungen.

Die EDV-Beauftragten dieses Clusters besitzen eine durchschnittliche Ressorterfahrung. Nahezu die Hälfte dieser Personen (46\%) verfügt über eine EDV-spezifische Berufsausbildung. Überdurchschnittliche Aktivitäten zur PC-spezifischen Aufgabenvorbereitung und Weiterbildung finden nicht statt. Fachliteratur ist diesbezüglich das einzig intensiver genutzte Hilfsmittel. Daneben existieren jedoch in hohem Maße freizeitinduzierte Kompetenzeffekte. So besitzen $68 \%$ aller EDV-Beauftragten auch im privaten Bereich einen Personal Computer.

\section{Reaktionsbezogene Kennzeichen:}

Die Einstellung zum Kundendienst ist in hohem Maße durch Zweifel und Skepsis geprägt. Vielfache Vorbehalte und Barrieren bestehen. Servicebefürwortende 
Argumente werden nicht angenommen. So ist nach Auffassung dieser Gruppe ein moderner DV-Einsatz ohne Kundendienst durchaus möglich. Sowohl der investitionsabsichernde Charakter als auch eine erhöhte Systemverfügbarkeit aufgrund von Serviceleistungen wird bestritten. Desweiteren zeigte sich, daß Eigenleistungen erheblich kostengünstiger sind als externer Kundendienst. Diesem mangelhaften Nutzenverständnis stehen durchschnittlich ausgeprägte Kundendiensthemmnisse gegenüber. Zusammenfassend werden somit Kundendienstvorteile intensiv bestritten, vordergründige Barrieren bestehen andererseits jedoch kaum.

Das Informationsverhalten zu PC- und servicebezogenen Sachverhalten ist insgesamt als durchschnittlich zu bezeichnen. Deutlich weniger genutzt werden jedoch Schulungen. Dies wird offenbar durch eine intensivere Literaturnutzung (Fachzeitschritten, -bücher) kompensiert.

In bezug auf das produktpolitische Kundendienstangebot weist die Gruppe ein sehr ausgeglichenes Profil auf. Die Bedeutung, die einzelnen Kundendiensten beigemessen wird, wird insgesamt sogar, vom Zufriedenheitsgrad, der bei der Inanspruchnahme einzelner Dienste erzielt wurde, übertroffen (vgl. Abbildung 26). Ausnahmen stellen hier lediglich die Hardware- und Betriebssysteminstallation, Schulungs- und Hot Line Dienste dar. Diese Situation ist jedoch vor dem Hintergrund zu sehen, daß das Erfahrungsniveau mit den verschiedenen Kundendienstprodukten insgesamt gesehen relativ niedrig ist.

Trotz der negativen Grundeinstellung stellen vorhandene Kundendienstmöglichkeiten ein zentrales Bewertungskriterium für die Anbieterwahl dar. Außergewöhnliche Erwartungen hinsichtlich distributionsbezogener Aspekte (Servicenetzdichte, Kundendienstkanäle u.a.) stellt das Segment nicht.

Auch besondere kontrahierungsbezogene Erwartungen an den Kundendienstanbieter bestehen nicht. Insgesamt werden die Preisniveaus des PC-Marktes (Hardware, Software, Service) jedoch eher als hoch eingestuft.

\section{Zusammenfassung:}

Personal Computer stellen im kundendienstskeptischen Segment nur eine Randerscheinung der betrieblichen Datenverarbeitungskonzeption dar. Fördernde Impulse ergeben sich vor allem von Anwenderseite. Das Interesse an diesen Systemen ist dabei in hohem Maße freizeitinduziert. 


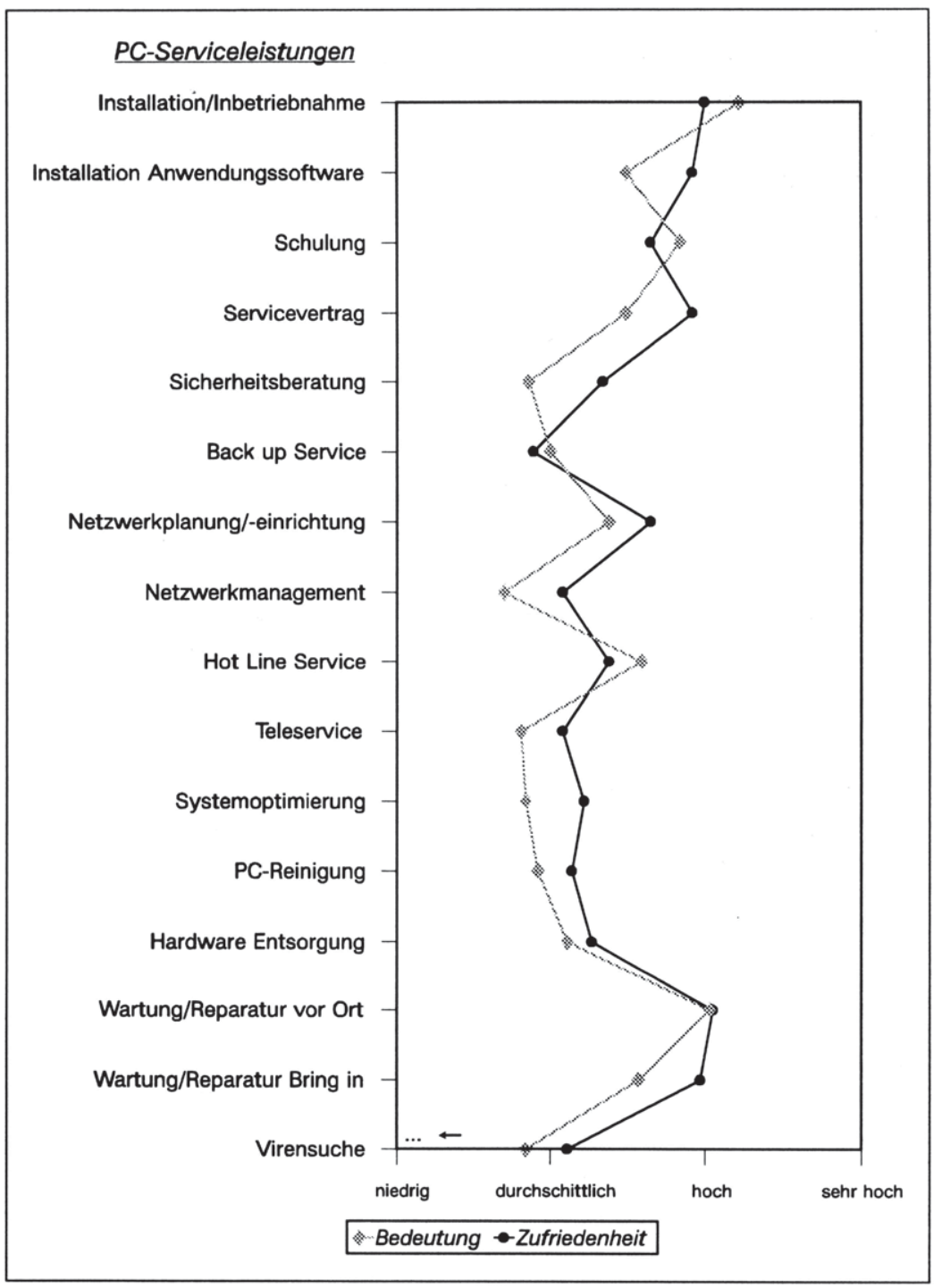

Abbildung 26: Kundendienstbezogene Bedeutungs- und Zufriedenheitsbeurteilung des kundendienstskeptischen Segments 
Kundendienst wird mit sehr großen Vorbehalten betrachtet. Ein zentraler Gesichtspunkt ist dabei vor allem, daß der Nutzen von PC-bezogenen Kundendienstleistungen bislang wenig verstanden wird. Eine forcierte Überzeugungsund Aufklärungsarbeit seitens der Anbieter ist gerade vor dem Hintergrund der größeren Betroffenheit (Stör- und Ausfallerfahrungen) erforderlich, um bestehende Vorurteile abzubauen und Nutzeneinsicht zu schaffen.

\subsection{Das kundendienstunbetroffene Segment}

\section{Bedürnisbestimmende Faktoren:}

Cluster $5(n=23)$ weist von allen Gruppen die geringste PC-Einbindung auf. Eine gerade noch durchschnittliche PC-Integration besteht in den Bereichen Produktund Außendienstwesen. Im Marketing sowie in Personal- und kaufmännischen Bereichen spielen Personal Computer keine Rolle. Die Ausfallsensibilität der Systeme wird trotzdem als hoch eingestuft. Offensichtlich werden dabei Bewertungsmaßstäbe anderer EDV-Systeme übertragen. Negative Erfahrungen mit Stör- und Ausfallsituationen bei PCs exitieren bislang nicht bzw. in nur ganz geringem Maße.

Die PC-Kompetenz im Unternehmen ist eher niedrig einzustufen. Ganz gering zeigt sich das Involvement der EDV-Beauftragten. Auch die Geschäftsleitung besitzt diesbezüglich ein unterdurchschnittliches Know how. Die einzigen positiven Kompetenzeffekte ergeben sich im Bereich der heuristischen Anwenderkompetenz. Programmierkenntnisse stehen in durchschnittlichem Umfang zur Verfügung. Die Unternehmen besitzen mittlere DV-Erfahrungen.

\section{Makro- und mikroanalytische Kriterien:}

Die Unternehmen des Segments weisen kein charakteristisches Branchenbild auf. Ansatzweise handelt es sich eher um Industrie-, weniger um Dienstleistungsunternehmen. Diese Betriebe beschäftigen durchschnittlich mehr Mitarbeiter als andere Segmente. Dabei ist der Mitarbeiteranteil, der im Rahmen seiner Tätigkeit mit Personal Computern arbeitet mit $9 \%$ am kleinsten. Nahezu alle Gruppenmitglieder (91\%) setzen zusätzlich auch noch andere Computersysteme ein. Vornehmlich handelt es sich dabei um mittlere Rechnersysteme mit herstellerspezifischer Betriebssystemsoftware (proprietary Systeme). Die Bedeutung von Personal Computern für den Unternehmenserfolg ist ebenso wie deren Stellenwert im Rahmen der gesamten EDV-Lösung gering. Die Systeme besitzen für die 
betrieblichen Arbeitsabläufe nur geringe Relevanz. Sie stellen damit ein ersetzbares, allenfalls operatives Arbeitsmittel dar.

Cluster 5 nutzt ausschließlich reine Standardkonfigurationen. Spezielle Lösungsund Individualsoftware wird nicht eingesetzt. Auch hardwarebezogen läßt sich kein typisches Konfigurationsbild erkennen.

Beim Hauptleistungskauf werden umfangreiche Grundservicevereinbarungen getroffen. Im einzelnen handelt es sich dabei um Installationsdienste, Einweisungen, Serviceverträge und Gewährleistungsvereinbarungen. Einzige Ausnahme stellen Schulungen dar. Sie werden verhältnismäßig wenig in Anspruch genommen.

Die EDV-Beauftragten besitzen eine vergleichsweise durchschnittlich lange $\mathrm{Er}$ fahrung und sind tendenziell älter als ihre Kollegen aus anderen Segmenten (45 Jahre). $30 \%$ von innen haben eine fachspezifische Berufsausbildung. Zusätzliche Anstrengungen zu einem PC-spezifischen Aufgabenvorbereitungs- und Weiterbildungsverhalten werden nur in sehr begrenztem Maße gemacht. Insbesondere Schulungen werden sehr wenig in Anspruch genommen. Lediglich $30 \%$ der EDV-Beauftragten beschäftigen sich auch zu Hause mit Personal Computern.

\section{Reaktionsbezogene Kennzeichen:}

Das kundendienstpolitische Einstellungsbild wird in entscheidendem Maße von dem Vorurteil "moderne Hardware braucht keinen Service" bestimmt. Zwar erfültt Kundendienst grundsätzlich eine Investitions-Absicherungsfunktion. In bezug auf Personal Computer besteht jedoch kein Servicebedarf. Darüber hinaus sind Eigenleistungen billiger. Die Inanspruchnahme von Leistungen ist in erster Linie auf Empfehlungen der eigenen Anwender zurückzưühren.

Diesen erheblichen Kundendienstbarrieren steht das vergleichsweise schwächste PC-bezogene Informationsverhalten aller Cluster gegenüber. Die EDV-Beauftragten nutzen weder Fachzeitungen und -literatur noch Schulungen, Tagungen sowie Anbieterinformationen. Einzige Informationsquelle ist der gegenseitige Kollegenkontakt. Entsprechend gering ist auch die Bekanntheit einzelner Serviceprodukte.

Die Kundendienstbedeutung wird durchschnittlich eingestuft. Abgesehen von Hardware- und Betriebssysteminstallationen sowie Hot Line Diensten, entspricht die Zufriedenheit mit den Serviceergebnissen den aufgestellten Erwartungen (vgl. Abbildung 27). 
PC-Serviceleistungen

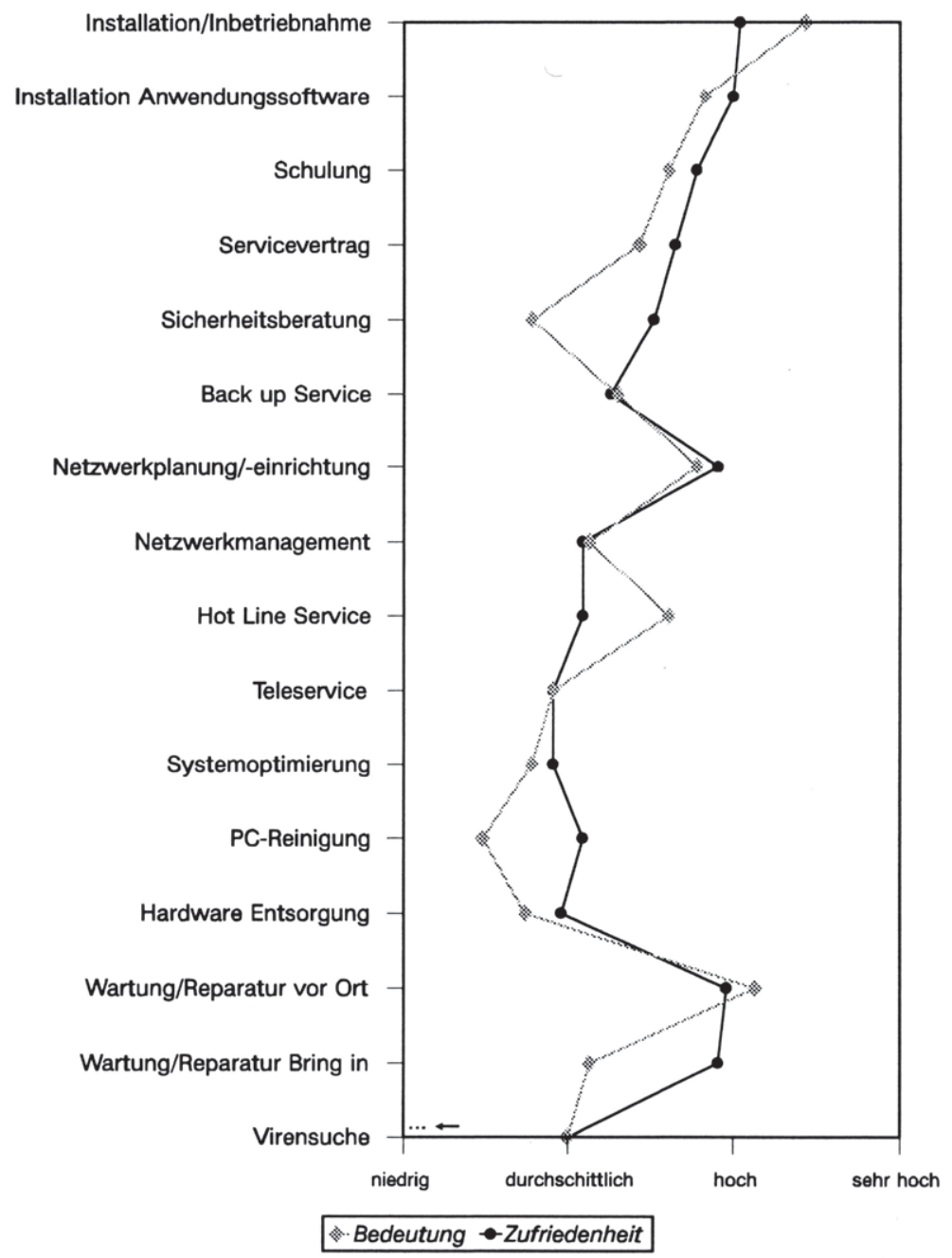

Abbildung 27: Kundendienstbezogene Bedeutungs- und Zufriedenheitsbeurteilung des kundendienstunbetroffenen Segments 
Außergewöhnlich zufrieden waren die Unternehmen dieses Clusters mit Sicherheitsberatungen, PC-Reinigungen und Bring-in Diensten.

Das kundendienstunbetroffene Segment stellt vergleichsweise wenig restriktive Anforderungen an seine Serviceanbieter. Weder ein umfangreiches Serviceabgebot, noch 24-StundenDienste werden erwartet.

Auch die distributionsbezogenen Erwartungen sind verhältnismäßig schwach ausgeprägt. Weder innovative Kundendienstkanäle und -infrastrukturen noch eine hohe Servicenetzdichte werden von Cluster 5 gefordert.

In bezug auf kontrahierungsbezogene Aspekte wird besonders ein zu hohes Servicepreisniveau kritisiert. Überzogene Forderungen führen vielfach zum Serviceverzicht bzw. u.U. zum Anbieterwechsel.

\section{Zusammenfassung:}

Personal Computer werden als strategisches DV-Instrument für Unternehmen bislang nicht akzeptiert und spielen in Cluster 5 keine Rolle. DV-Aufgaben werden weitestgehend mit Hilfe anderer EDV-Systeme vollzogen. Infolgedessen sind die Unternehmen von PC-bezogenen Kundendienstfragen auch weitestgehend unbetroffen. Darüber hinaus bestehen vielfache Bedenken und Barrieren über den Sinn und den Nutzen von PC-Services. Die insgesamt ablehnende Haltung gegenüber Personal Computern führt in der Konsequenz zu einer entsprechenden Zurückweisung diesbezüglicher Kundendienste.

\subsection{Das kundendienstignorante Segment}

\section{Bedürfnisbestimmende Faktoren:}

Cluster $6(n=29)$ weist eine sehr starke PC-Abhängigkeit im kaufmānnischen Wesen auf. Die Integration in anderen Unternehmensbereichen ist, mit Ausnahme einer niedrigen Außendiensteinbindung, durchschnittlich. Dies trifft ebenfalls auf die Ausfallsensibilität der Systeme zu. Stör- und Ausfallsituationen sind hingegen bislang verhältnismäßig häufig aufgetreten.

PC-Kompetenz wird im Unternehmen durch ein starkes Involvement der Geschäftsführer und ein hohes Know how der Anwender verkörpert. Neben operativen Fähigkeiten besitzen diese Mitarbeiter auch eine hohe epistemische PCKompetenz. Ferner bestehen verhältnismäßig gute Programmierkenntnisse. Die 
Kompetenz des EDV-Beauftragten und der Abteilungsleiter liegt im Durchschnitt. Freizeitinduzierte Erfahrungseffekte

ergeben sich nicht. Insgesamt gesehen besteht eine relativ kurze PC-Erfahrung.

\section{Makro- und mikroanalytische Kriterien:}

Bei den Unternehmen dieses Clusters handelt es sich nahezu ausschließlich um Handelsbetriebe. Die Mitarbeiterzahl ist im Vergleich zu den übrigen Segmenten deutlich kleiner. Nur 34\% der Unternehmen setzen zusätzlich andere EDV-Systeme ein. Auch dies ist im Verhältnis zu einer Gesamtbetrachtung die kleinste Ausprägung. Sowohl die Bedeutung von Personal Computern für den Unternehmenserfolg als auch deren Stellenwert im Rahmen der gesamten EDV-Lösung wird nur durchschnittlich bewertet. Dennoch ist zu berücksichtigen, daß mit Hilfe von Personal Computern zentrale Aufgaben der betrieblichen Datenverarbeitung durchgeführt werden. Aus diesem Grund ist der Charakter dieser Systeme letztendlich dennoch als strategisch bedeutsam einzustufen.

Die PC-Konfigurationen sind in diesem Segment relativ stark durch den Einsatz von Individualsoftware gekennzeichnet. Typische Standardanwendungen werden vergleichsweise wenig genutzt. Hardwarebezogen läßt sich kein typisches Konfigurationsbild identifizieren.

Mit Ausnahme besonderer Gewährleistungsvereinbarungen werden beim Hauptleistungskauf umfangreiche Grundservices in Anspruch genommen. Im einzelnen handelt es sich besonders um Einweisungen, aber auch um Installationsdienste und Serviceverträge.

Die EDV-Beauftragten besitzen eine durchschnittlich lange Erfahrung. Lediglich $21 \%$ haben eine fachbezogene Berufsausbildung. Instrumente zur PC-spezifischen Aufgabenvorbereitung und Weiterbildung werden sehr differenziert in Anspruch genommen. Überdurchschnittlich präferiert werden Herstellerschulungen. Darüber hinaus besteht ein intensiver Kollegenkontakt. Literatur, Fachseminare sowie das learning-by-doing-Prinzip kommen vergleichsweise wenig zur Anwendung. Nur 34\% der EDV-Beauftragten besitzen auch im privaten Umfeld einen PC.

\section{Reaktionsbezogene Kennzeichen:}

Das Einstellungsprofil in bezug auf Kundendienstleistungen ist relativ undifferenziert. Es bestehen weder eindeutige Servicemotive noch -barrieren. Die Haupt- 
gründe Service in Anspruch zu nehmen, sind entsprechende Empfehlungen der eigenen Anwender. Auch Verkäuferempfehlungen werden berücksichtigt. Vorbehalte bestehen in bezug auf Datenschutzaspekte.

Das Informationsverhalten der EDV-Beauftragten zu PC-bezogenen Themen ist sehr differenziert und eher auf persönliche Kommunikation ausgerichtet. Vergleichsweise wenig genutzte Quellen sind Tagungen, Fachzeitschriften und -literatur. Relativ intensiv wird dagegen der direkte Informationsaustausch gesucht. Dies geschieht sowohl mit Herstellern, als auch mit Wettbewerbern und Kollegen des eigenen Unternehmens. Berichte und Informationen im Fernsehen bzw. Radio ergänzen das Spektrum.

Die Serviceproduktbekanntheit wie auch die Erfahrungen mit verschiedenen Kundendiensten sind insgesamt durchschnittlich. Dies trift auch auf die Bedeutung zu, die einzelnen Leistungen beigemessen wird (vgl. Abbildung 28). Abgesehen von Diensten der Hardware- und Betriebssysteminstallationen, der Hardware Entsorgung sowie des Hot Linie Service, entsprechen die Kundendiensterfahrungen den aufgestellten Erwartungen. Außergewöhnlich zufrieden waren die Unternehmen dieses Clusters besonders mit Wartungs- und Reparaturservices.

Über diese Gesichtpunkte hinaus legt die Gruppe besonderen Wert auf ein umfangreiches Serviceangebot, eine 24-stündige Kundendiensterreichbarkeit und eine 24-stündige Technikerverfügbarkeit. Dies korrespondiert mit den umfangreichen Ausfallerfahrungen, die die Bedeutung dieser spezifischen Kundendienstleistungen für das Unternehmen evident machen.

In bezug auf distributionsbezogene Gesichtspunkte fordern die Unternehmen eine hohe Servicenetzdichte. Das gegenwärtige Preisniveau für Kundendienst wird überwiegend akzeptiert. Ungerechtfertigte und überzogene Forderungen dagegen, führen u.U. zum Serviceverzicht bzw. zum Anbieterwechsel.

\section{Zusammenfassung:}

Personal Computer übernehmen im kaufmännischen Bereich wesentliche EDVAufgaben in Cluster 6 . In vielen Fällen führten Systemausfälle bereits zu Störungen der Betriebsabläufe. Da vergleichbare Alternativtechnologien kaum zur Verfügung stehen gewinnt dieser Aspekt zunehmend an Gewicht. 


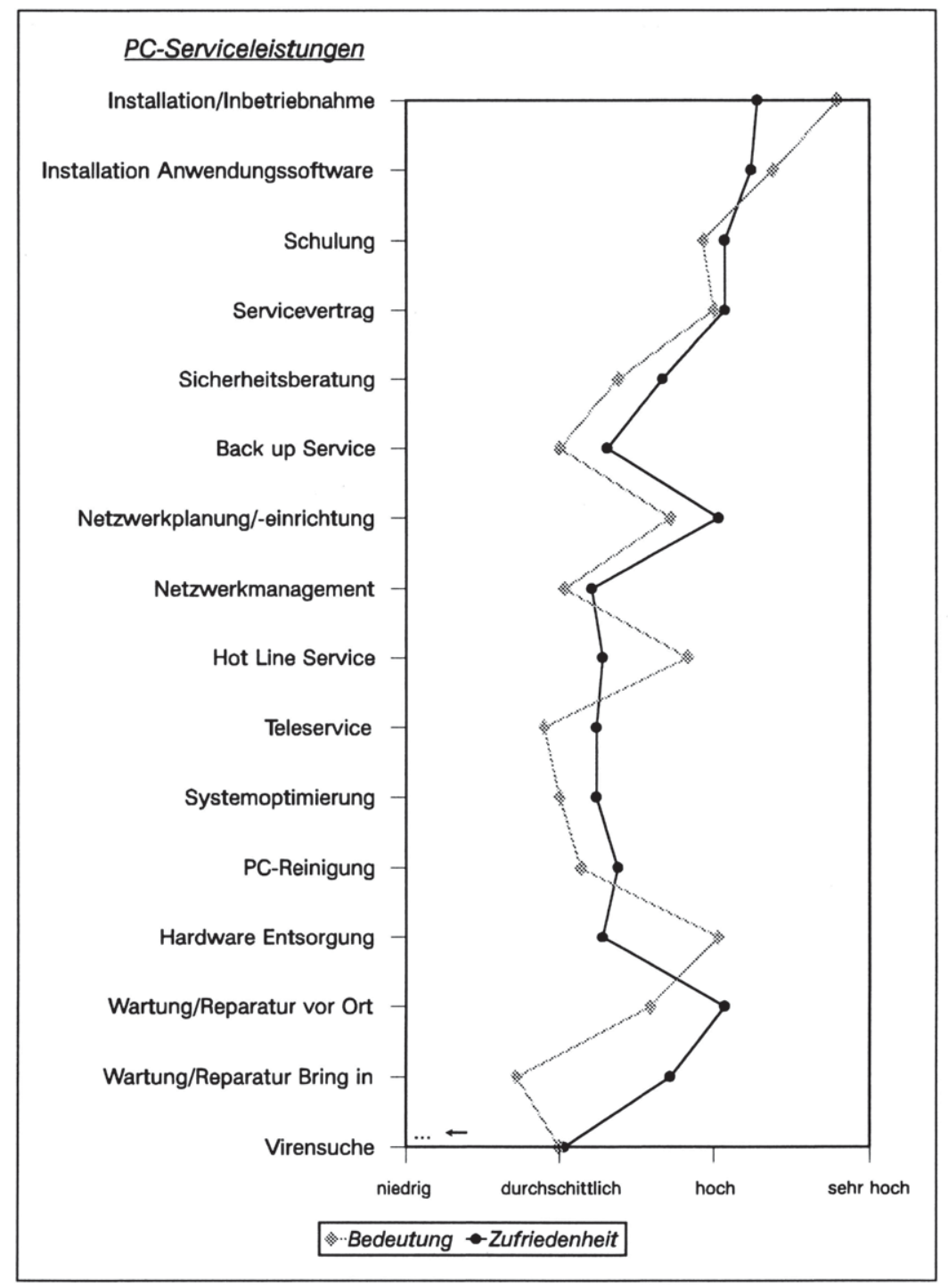

Abbildung 28: Kundendienstbezogene Bedeutungs- und Zufriedenheitsbeurteilung des kundendienstignoranten Segments 
Mangelnde Aufgeklärtheit bzw. fehlende Erfahrungen (z.B. Verlust wichtiger Daten, Virenbefall) veranlaßte die Unternehmen dieses Segment bislang dazu, mögliche Gefahren der PC-spezifischen Datenverarbeitung zu unterschätzen. Die Möglichkeiten des Kundendienstes werden als probates Mittel zur Risikoreduktion bislang noch ignoriert Eine Thematisierung des Servicenutzens hat noch nicht bzw. zu wenig stattgefunden.

\subsection{Trennschärfe der Kundendienstsegmente}

In einem dritten Analyseschritt sind die Unterschiede zwischen den identifizierten Kundendienstsegmenten zu überprüfen. Als methodisches Instrumentarium bietet sich die Diskriminanzanalyse an. ${ }^{182}$ Hierbei werden die segmentbildenden Faktoren nach ihrer Trennfähigkeit in die Diskriminanzanalyse einbezogen. Die Güte der Diskriminanzanalyse läßt sich mit Hilfe des Wilks' Lambda und des Anteils korrekter Klassifikation ermitteln. Wilks' Lambda kann Werte zwischen 0 und 1 annehmen und gilt als Maß für die Unterschiedlichkeit der Untersuchungsgruppen. Je kleiner der Wert in der Untersuchung ist, desto größer sind die Unterschiede zwischen den voneinander zu trennenden Gruppen. Der Anteil der richtigen Klassifikation gibt Auskunft über die Prognosefähigkeit der ermittelten Diskriminanzfunktion(en). Der Erfolg der Diskriminierung steigt mit der Höhe der richtigen Klassifikation. 183 Für die vorliegende Untersuchung bietet sich die Diskriminanzanalyse vor allem deshalb an, um die Trennungsfähigkeit der operationalisierten Bedürfnisdimensionen des Kundendienstes zu verifizieren. Von daher wird die Diskriminanzanalyse über alle clusterbildenden Faktoren durchgeführt.

Im einzelnen konnten 5 Diskriminanzfunktionen ermittelt werden, die jeweils hoch signifikant sind $(\alpha \leq 0,01)$. Die ermittelten Wilks' Lambda Werte liegen im

182 Zur Diskriminanzanalyse vgl. Backhaus, K., et al., Multivariate Analysemethoden, a.a.O., S. 161 ff.; Meffert, H., Marketingforschung und Käuferverhalten, a.a.O., S. 279 ff.

183 Vgl. Büker, B., Qualitätsbeurteilung investiver Dienstleistungen, a.a.O., S. 159. 
Rahmen vergleichbarer Untersuchungen. ${ }^{184}$ Sie dokumentieren damit eine befriedigende Trennkraft der ermittelten Diskriminanzfunktionen. 185

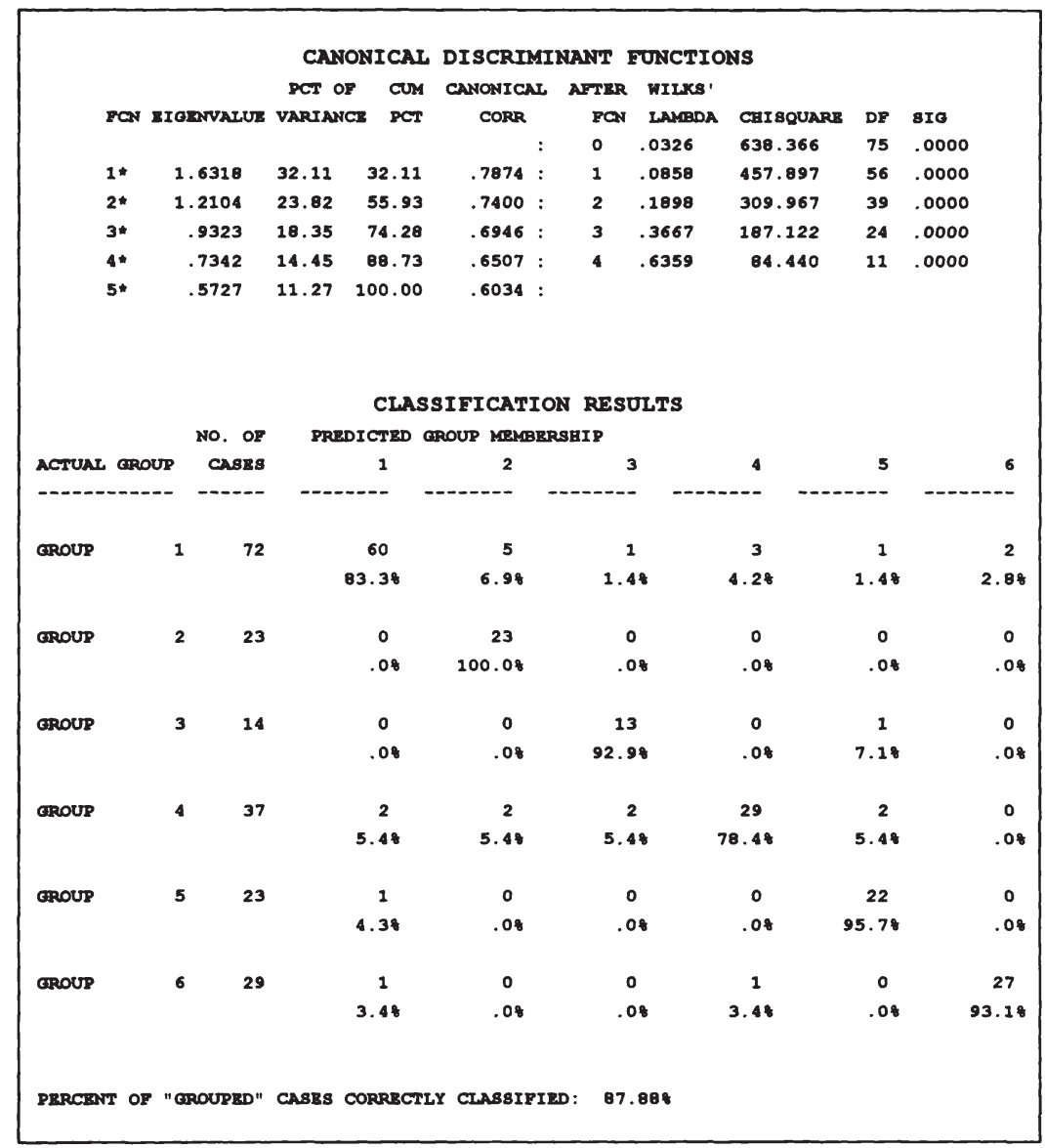

Abbildung 29: Gütemaße der Diskriminanzfunktion (SPSS-Tableau)

184 Vgl. Heinemann, G., Betriebstypenprofilierung und Erlebnishandel, Wiesbaden 1989, S. 224, Tab. 21.

185 Die ersten beiden Funktionen weisen einen Wilks' Lambda-Wert unter 0,1 (0,0326 bzw. 0,0858 ) auf. Die dritte Funktion erzielt einen Wert von 0,1898 , und die vierte bzw. fünfte Funktion ein Niveau von 0,3667 bzw. 0,6359. Vgl. hierzu die Spalte WILKs' LAMBDA in der Tabelle CanONICAL DISKRIMINANT FUnCtions in Abbildung 29. 
Auch die Wahrscheinlichkeit einer korrekten Klassifikation kann mit einem Wert von $87,88 \%$ überzeugen. Dieses Ergebnis besagt, daß die auf Grundlage der Diskriminanzwerte prognostizierte Zugehörigkeit der befragten Unternehmen zu einem bestimmten Kundendienstsegment zu 87,88\% mit der tatsächlichen Gruppenstruktur übereinstimmt. Abbildung 29 stellt die Gütemaße der Diskriminanzfunktion sowie das Klassifikationsergebnis der Kundendienstsegmente dar.

Eine weitere Erkenntnis, die mit Hilfe der Diskriminanzanalyse gewonnen werden kann, ist die jeweilige diskriminatorische Bedeutung der einzelnen Bedürfnisfaktoren. Hierzu sind die mit den Eigenwertanteilen gewichteten absoluten Werte der standardisierten Diskriminanzfunktionskoeffizienten einer Variablen zu addieren. 186 Das Ergebnis stellt die mittleren Diskriminanzkoeffizienten dar, die in Tabelle 5 dargestellt sind. 187

Die relative Bedeutung der diskriminierenden Faktoren liegt zwischen $4 \%$ und $11 \%$, d.h. daß zunächst alle Faktoren einen relativ homogenen Unterscheidungsbeitrag für die einzelnen Kundendienstsegmente leisten.

Das wichtigste Trennungskriterium stellt das PC-Involvement der EDV-Beauftragten dar. Auf dieses Element fallen $10,95 \%$ der erklärten Varianz. Dieses Kriterium bildet den wichtigsten Indikator für den Differenzierungsgrad von Servicegruppen und dokumentiert somit erneut die bedürfnisprägende Bedeutung dieses Personenkreises für investive PC-Kundendienste. Darüber hinaus besitzen die Faktoren PC-Abhängigkeit im Produktwesen (9,42\%), Programmierkenntnisse $(8,86 \%)$ und DV-Erfahrung der Unternehmen $(7,61 \%)$ hohe diskriminatorische Relevanz.

Demgegenüber liefern die Kriterien Stör- und Ausfallerfahrungen (4,65\%), heuristische Anwenderkompetenz (4,59\%) sowie die PC-bezogene Ausfallsensibilitāt $(4,38 \%)$ geringere Beiträge zur Segmentdifferenzierung.

186 Vgl. zu dieser Vorgehensweise insbesondere Backhaus, K., et al., Multivariate Analysemethoden, a.a.O., S. 201.

187 Die standardisierten Diskriminanzkoeffizienten der einzelnen Faktoren sind der Tabelle Al-1 im Anhang dieser Arbeit zu entnehmen. 


\begin{tabular}{|r|l|c|c|}
\hline Rang & \multicolumn{1}{|c|}{ Diskriminierende Faktoren } & $\begin{array}{c}\text { Mittlere } \\
\text { Diskriminanzfunk- } \\
\text { tionskoeffizienten }\end{array}$ & $\begin{array}{c}\text { Pelative Bedeutung } \\
\text { der mittleren Diskri- } \\
\text { minanzfunktions- } \\
\text { koeffizienten (in \%) }\end{array}$ \\
\hline 1. & PC-Involvement EDV-Beauftragter & 0,4078 & $10,95 \%$ \\
2. & PC-Abhängigkeit im Produktwesen & 0,3509 & $9,42 \%$ \\
3. & Programmierkenntnisse & 0,3297 & $8,86 \%$ \\
4. & DV-Erfahrungen & 0,2833 & $7,61 \%$ \\
5. & epistemische Anwenderkompetenz & 0,2679 & $7,20 \%$ \\
6. & Erfahrungen durch Freizeitbetrieb & 0,2655 & $7,13 \%$ \\
7. & PC-Involvement Geschäftsführer & 0,2539 & $6,82 \%$ \\
8. & PC-Abhängigkeit im Außendienstwesen & 0,2281 & $6,13 \%$ \\
9. & PC-Abhängigkeit im Personalwesen & 0,2210 & $5,94 \%$ \\
10. & PC-Involvement Abteilungsleiter & 0,2154 & $5,79 \%$ \\
11. & PC-Abhängigkeit im kfm. Wesen & 0,2104 & $5,65 \%$ \\
12. & PC-Abhängigkeit im Marketing & 0,1822 & $4,89 \%$ \\
13. & Stör- und Ausfallerfahrungen & 0,1733 & $4,65 \%$ \\
14. & heuristische Anwenderkompetenz & 0,1708 & $4,59 \%$ \\
15. & PC-bezogene Ausfallsensibilität & 0,1630 & $4,38 \%$ \\
\hline$\Sigma$ & & 3,7232 & $100 \%$ \\
\hline
\end{tabular}

Tabelle 5: Diskriminatorische Bedeutung segmentbildender Faktoren

Im Gesamtergebnis kann festgehalten werden, daß die auf Grundlage der Bedürfnisdimensionen ABHÄNGIGKEITSGRAD und KUNDENKOMPETENZ durchgeführte Gruppenbildung zu sechs, inhaltlich plausiblen und in hohem Maße trennscharfen Kundendienstsegmenten geführt hat. Damit ist die Existenz unterschiedlicher Bedürnnisgruppen im Servicemarkt nachgewiesen. Hypothese $\mathbf{H}_{\mathbf{S}} \mathbf{2}$ ist damit verifiziert. 


\section{Die Analyse segmentspezifischer Machtstrukturen bei kundendienst- bezogenen Kaufentscheidungsprozessen}

Die bisherigen Untersuchungen haben Unterschiede in der kundendienstpolitischen Bedürfnislage von Unternehmen bestätigt. Dagegen ist bislang ungeklärt, inwieweit die durch die EDV-Beauftragten definierten Bedürfnisanforderungen auch im Rahmen der Kaufentscheidungsprozesse ihre Umsetzung in tatsächliche Servicenachfrage finden.

Entscheidend hierfür ist der Einfluß und die Durchsetzungskraft dieser Personen in kundendienstbezogenen Kaufentscheidungssituationen. Werden ihre Vorstellungen und Anregungen weitestgehend übernommen, müssen sich die Schwerpunkte der kundendienstpolitischen Marktbearbeitung daran orientieren. Bestehen dagegen wesentliche Einflüsse anderer Buying Center Mitglieder, so wird sich das bislang bestehende Bedürfnisprofil einzelner Segmente u.U. erheblich verändern. Derart auftretende Gaps zwischen Bedürfnissituation und Entscheidungsrealitāt erfordern eine veränderte Gestaltung der kundendienstorientierten Marktbearbeitung. Dies gilt insbesondere für die Entwicklung kommunikationsbezogener Strategien. Die fünfte und sechste Untersuchungshypothese werden gemeinsam untersucht und lauten:

$\mathbf{H}_{\mathbf{M}} \mathbf{1}$ : Die Strukturen von kundendienstbezogenen Kaufentscheidungsprozessen sind vergleichbar zu denen von PC-Kăufen. Die Nutzung hauptleistungsbezogener Erfahrungen führt von daher zur Erzielung wesentlicher Synergieeffekte im Rahmen der kundendienstbezogenen Marktbearbeitung.

$\mathbf{H}_{\mathbf{M}}$ : Die Machtstrukturen in bezug auf kundendienstbezogene Kaufentscheidungen sind segmentübergreifend unterschiedlich ausgeprägt. Innerhalb einzelner Bedürfnissegmente lassen sich jedoch typische Entscheidungsstrukturen identifizieren.

Zur Analyse dieser Fragestellungen, werden die Entscheidungs-, Beeinflussungs- und Anregungsstrukturen in den einzelnen Segmenten jeweils tür PCund für Kundendienstkäufe getrennt untersucht. Dabei sind diejenigen Personen zu identifizieren, die in den jeweiligen Kaufentscheidungsprozessen die Funktion des "Macht-" und "Fachpromotors" sowie des "Initiators" verkörpern. 
Im einzelnen wird zur Analyse dieser Fragestellungen die Bedeutung von Geschäftsführern, EDV-Beauftragten, Abteilungsleitern und Anwendern insgesamt sowie differenziert in den sechs Kundendienstsegmenten näher untersucht. ${ }^{188}$

\subsection{Machtpromotoren bei Kundendienstkaufsituationen}

Im Rahmen eines ersten Untersuchungsschrittes werden die Machtpromotoren bei Kundendienstkäufen identifiziert. ${ }^{189}$ Im Mittelpunkt der Betrachtung stehen somit diejenigen Personen in kundendienstbezogenen Kaufentscheidungsprozessen, die das Ergebnis der Überlegungen letztendlich festlegen bzw. bestimmen. Abbildung 30 kennzeichnet zunächst die gesamtmarktbezogenen Machtstrukturen. Gegenübergestellt werden dabei die Situation bei hauptleistungsund bei kundendienstspezifischen Kaufentscheidungen.

Die Analyse zeigt, daß PC- und kundendienstbezogene Kaufsituationen strukturell in hohem Maße vergleichbar sind. Zwar zeigt sich im Rahmen von Servicekäufen eine geringfügige Übertragung geschäftsführerspezifischer Entscheidungsbefugnisse auf EDV-Beauftragte. Die dominierende Rolle der Geschäftsleitung wird hierdurch jedoch nicht gemindert. Sie verkörpern in beiden Prozessen die Rolle der Machtpromotoren. Eine gewisse Bedeutung kommt darüber hinaus auch noch den EDV-Beauftragten zu. Immerhin jede fünfte PC- bzw. jede dritte Kundendienstentscheidung wird von ihnen angeordnet. Abteilungsleiter und besonders Anwender spielen bei der Entscheidungsverabschiedung kaum eine bzw. keine Rolle.

Die Analyse der Entscheidungsmacht in den einzelnen Segmenten führt zu ambivalenten Ergebnissen (vgl. im folgenden auch die Abbildungen 31a und 31b).

188 Da die Bedeutung von Kaufentscheidungen immer auch vom Investitionsvolumen abhängig ist, wurde die Buying Center Situation zunächst jeweils getrennt für größere und für kleinere Kaufsituationen erfaßt. Die semantische Unschärfe durch die Wortwahl "größer" und "kleiner" wurde dabei bewußt in Kauf genommen. So kann zwar unterstellt werden, daß jeder Befragte unterschiedliche Größenvorstellungen hinsichtlich der Attribute "größer" und "kleiner" besitzt. Wesentlich bedeutsamer erschien es im vorliegenden Fall jedoch, die einzelnen Auskunftspersonen dahingehend anzuhalten, Grenzbereiche im Buying Center-bezogenen Rollenverhalten (wichtige Entscheidung - weniger wichtige Entscheidung) zu kennzeichnen. Die Verdichtung beider Aussagen zu einer Gesamteinschätzung erfolgte schließlich durch die jewellige Verwendung des arithmetischen Mittels.

189 Zu den begrifflichen Grundlagen vgl. Kap. B.3.2. 
Dabei ist zunächst segmentübergreifend festzustellen, daß entsprechend der Gesamtmarktsituation die Geschäftsührer in allen Clustern entscheidungsdominant sind. So zeigt sich besonders im kundendienstunsensiblen, -unbetroffenen und im Standard Kundendienstsegment ein den Gesamtstrukturen vergleichbares Bild. Unterschiede ergeben sich jedoch in den Machtintensitäten der übrigen Cluster.

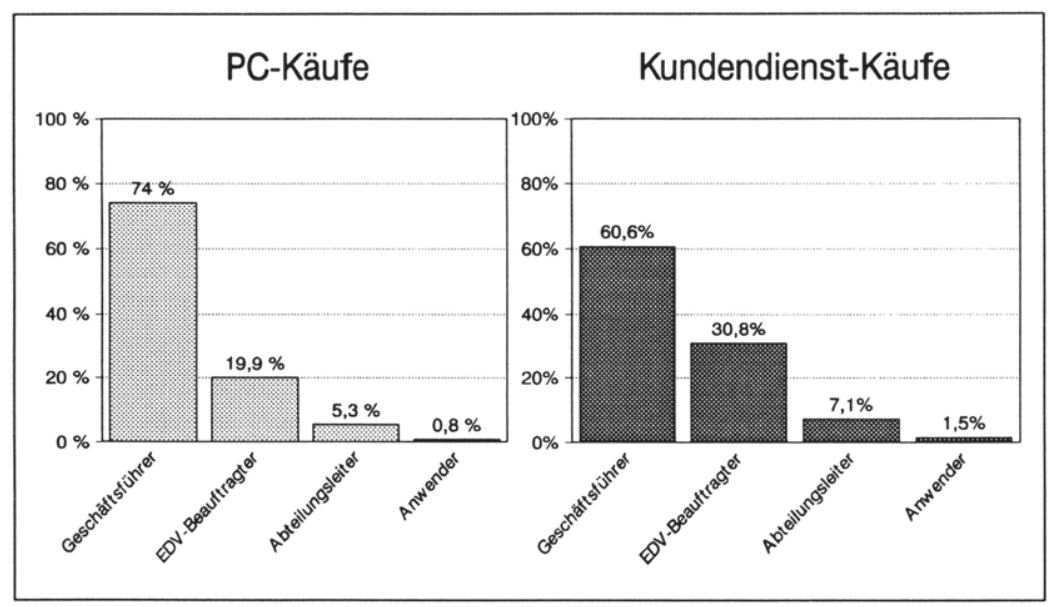

Abbildung 30: Entscheidungsmacht bei PC- und kundendienstbezogenen Kaufentscheidungen

Am stärksten ist dabei die Machtstellung der Geschäftsführer im kundendienstignoranten Segment ausgeprägt. 84,5\% dieser Personen bestimmen hier letztendlich die Servicenachfrage. Im Gegensatz zu allen anderen Teilmärkten konzentriert die Geschäftsleitung dabei ihre Entscheidungsmacht bei Kundendienstkäufen sogar noch stärker als bei PCs. Nur noch in $12 \%$ aller Fälle treffen die EDV-Beauftragten derartige Entscheidungen.

Demgegenüber lassen sich im kundendienstskeptischen und im Nischensegment eher multipersonale Machtstrukturen feststellen. So werden zwar in beiden Teilmärkten PC-bezogene Entscheidungen in erster Linie durch die Unternehmensspitze getragen. Bei Kundendiensten verteilen sich diese Befugnisse jedoch weitaus breiter. Hier relativiert sich die Machtpromotorenstellung zwischen der Geschäftsleitung und dem EDV-Beauftragten. Besonders im Nischensegment besitzen sie bei Servicekäufen nahezu die gleichen Entscheidungskompe 


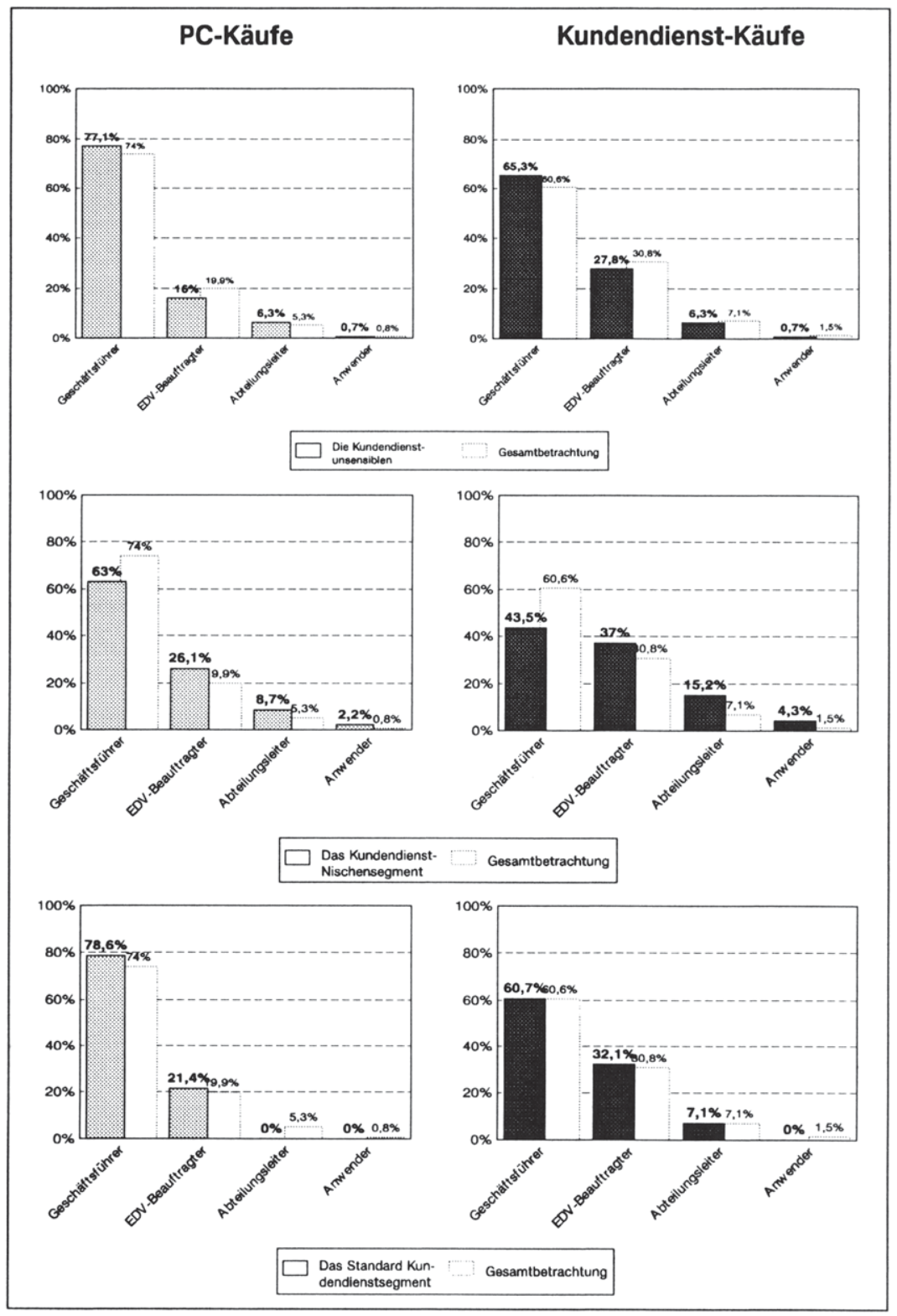

Abbildung 31a: Segmentspezifische Entscheidungsmacht bei PC- und kundendienstbezogenen Kaufsituationen (Segmente 1, 2 und 3) 


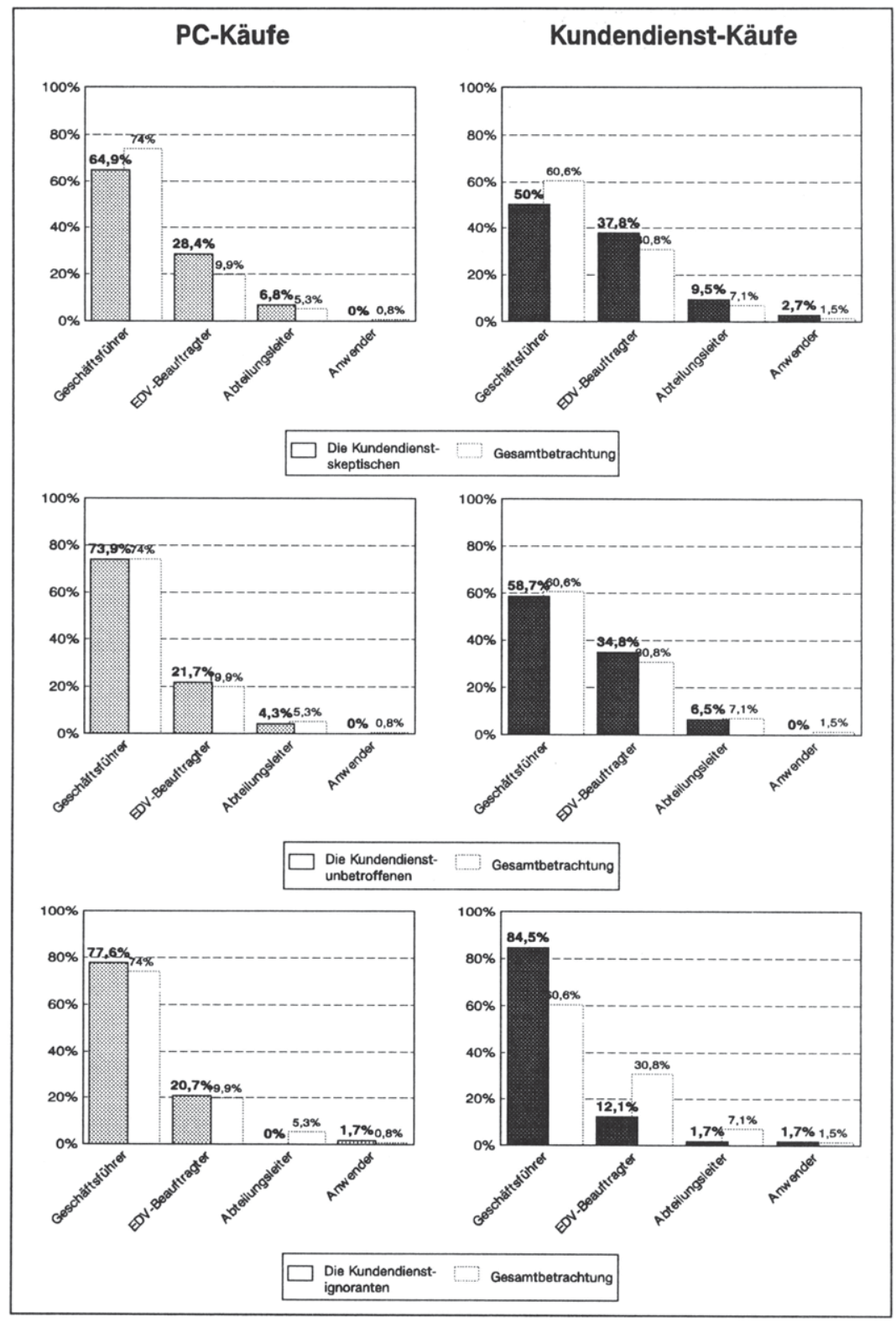

Abbildung 31b: Segmentspezifische Entscheidungsmacht bei PC- und kundendienstbezogenen Kaufsituationen (Segmente 4, 5 und 6) 
tenzen wie die Geschäftsführer. Ferner läßt sich mit $15,2 \%$ eine nennenswerte Relevanz der Abteilungsleiter feststellen. Vergleichbare Konstellationen, wenn auch nicht ganz so deutlich ausgeprägt, bestehen im kundendienstskeptischen Segment.

Als Zwischenergebnis kann damit festgehalten werden:

- Die Machtverhältnisse bei PC- und bei kundendienstbezogenen Kaufentscheidungsprozessen sind strukturell vergleichbar,

- Geschäftsführer besetzen die Funktion der Machtpromotoren in diesen Prozessen,

- bei Kundendiensten steigt im Verhältnis zu PC-Käufen die Entscheidungsbefugnis der EDV-Beauftragten,

- im kundendienstskeptischen und im Nischensegment existieren eher multipersonal gestreute Entscheidungskompetenzen,

- im kundendienstignoranten Segment werden Entscheidungsprozesse nahezu ausschließlich durch die Geschäftsleitung bestimmt.

\subsection{Fachpromotoren bei Kundendienstkaufsituationen}

In einem zweiten Untersuchungsschritt werden die Fachpromotoren von PCund kundendienstbezogenen Kaufentscheidungsprozessen identifiziert. $190 \mathrm{Ne}$ ben der Identifizierung letztendlicher Entscheidungsträger gilt es zu klären, welche Personen in den betrachteten Entscheidungsprozessen ursächlich meinungsbildend sind. Wiederum erfolgt zunächst eine gesamtmarktbezogene Betrachtung der PC- und kundendienstspezifischen Situation (vgl. Abbildung 32).

Die Analyse zeigt, daß in beiden Entscheidungsvorgängen tatsächliche Gruppenprozesse stattinden. Zur Meinungsbildung werden, wenn auch in unterschiedlichem Umfang, alle vier Personengruppen herangezogen. Selbst die Anwender, die bei der Entscheidungsverabschiedung keine Rolle spielen, besitzen noch gewissen Einfluß.

$190 \mathrm{Zu}$ den begrifflichen Grundlagen vgl. Kap. B.3.2. 


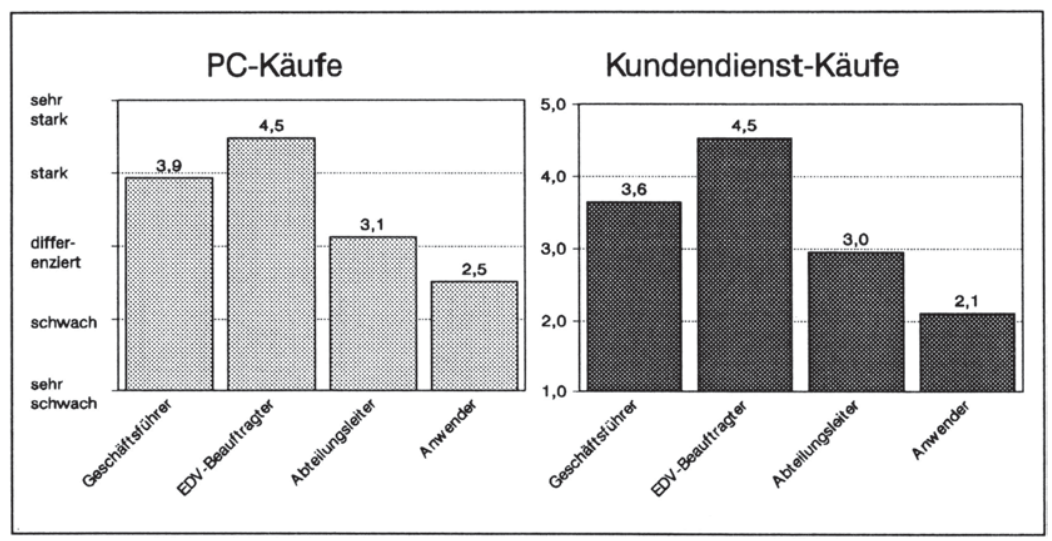

Abbildung 32: Entscheidungseinfluß bei PC- und kundendienstbezogenen Kaufentscheidungen

Eine zweite wesentliche Erkenntnis dokumentiert sich durch den Vergleich beider Situationen. Die Struktur der Entscheidungsentwicklung bei PC-Investitionen ist in hohem Maße mit der von Kundendienstkäufen vergleichbar. Die Bedeutung des EDV-Beauftragten und der Abteilungsleiter ist in beiden Fällen jeweils nahezu identisch. Eine geringfügige Abnahme des argumentativen Einflusses findet bei Kundendienstinvestitionen seitens der Geschäftsführer und Anwender statt.

Betrachtet man die Intensität der Beeinflussung, so zeigt sich die dominierende Stellung des EDV-Beauftragten. Er verkörpert in beiden Entscheidungsprozessen die Funktion des Fachpromotors. Sein hoher Sachverstand und seine fachspezifischen Erfahrungen stellen das zentrale Element im Meinungsbildungsprozess solcher Kaufsituationen dar. Starken Anteil an diesen Diskussionen besitzen auch die Geschäftsführer. Dies impliziert eine ausgewogene Gegenüberstellung technologiespezifischer und betriebswirtschaftlicher Gesichtspunkte. Die Einflußnahme der Abteilungsleiter ist in differenziertem Umfang in beiden Prozessen vorhanden. Auch Anwender finden in schwächerem Umfang noch Gehör.

Eine Analyse des Meinungsbildungsverhaltens der Buying Center in den einzelnen Segmenten bestätigt im wesentlichen die gesamtmarktbezogene Situation (vgl. im folgenden auch die Abbildungen 33a und 33b). Dies betrifft besonders 


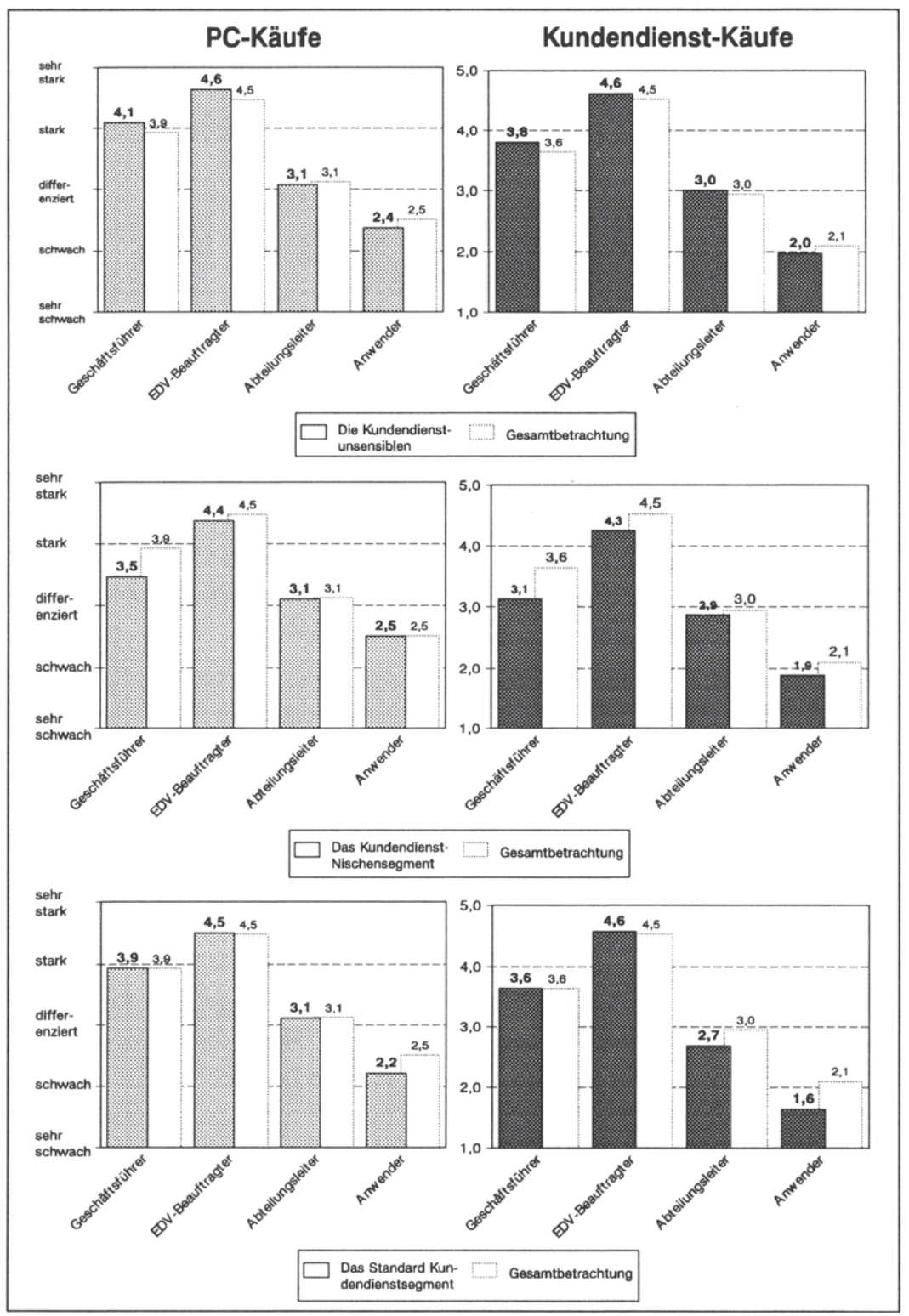

Abbildung 33a: Segmentspezifische Einflußstrukturen bei PC- und kundendienstbezogenen Kaufentscheidungsprozessen (Segmente 1, 2 und 3) 


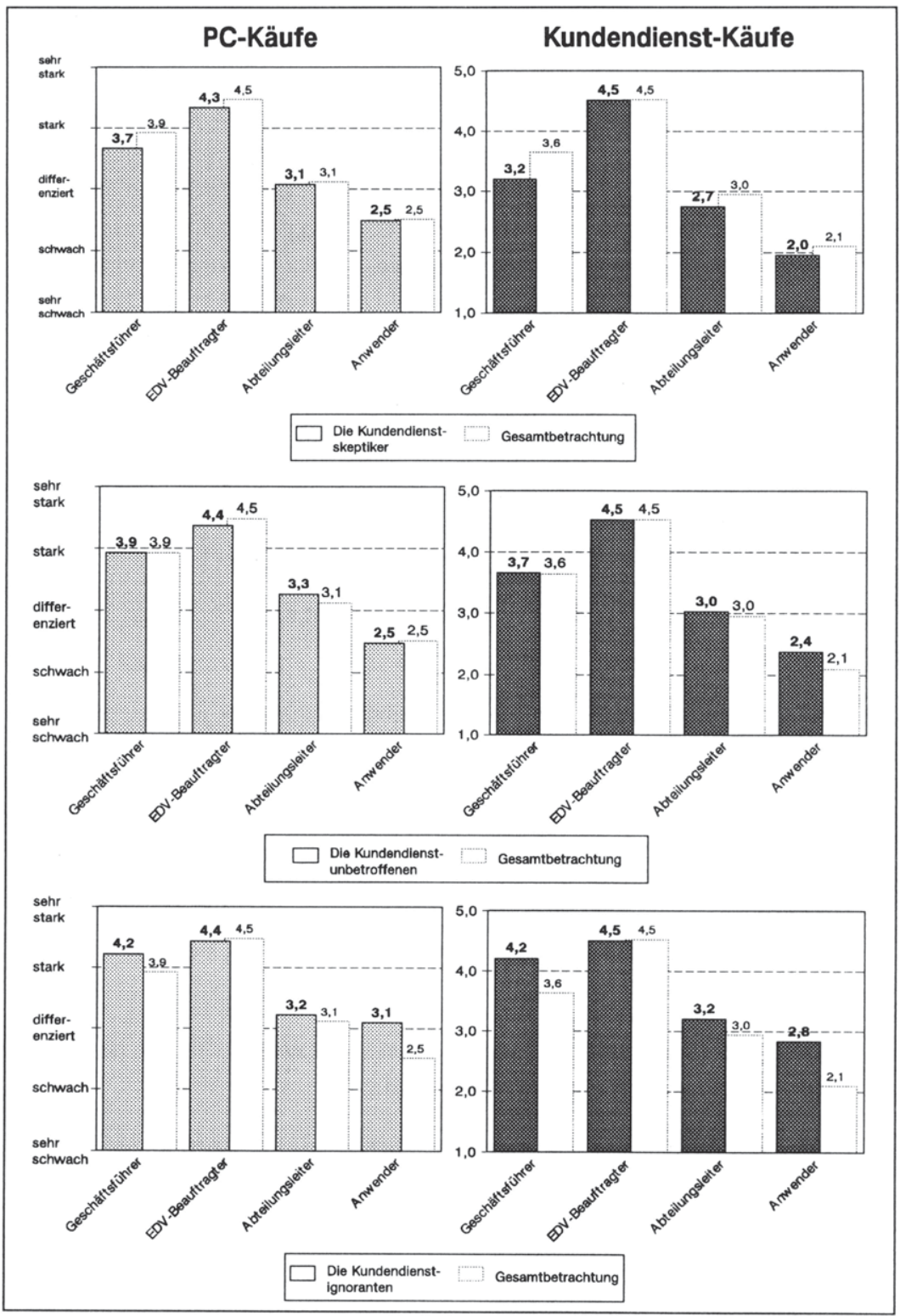

Abbildung 33b: Segmentspezifische Einflußstrukturen bei PC- und kundendienstbezogenen Kaufentscheidungsprozessen (Segmente 4, 5 und 6) 
das Segment der Kundendienstunsensiblen, das Standard Kundendienstsegment sowie das kundendienstunbetroffene Segment.

Unterschiede offenbaren sich dagegen im Nischensegment und im kundendienstskeptischen Segment. Insgesamt ist hier die Diskussionsintensität bei der Entscheidungsfindung geringer ausgeprãgt. Bei PC-Fragen findet ein intensiverer Meinungsaustausch zwischen dem EDV-Beauftragten und dem Geschäftsführer statt. Abteilungsleiter und Anwender sind in differenziertem Umfang beteiligt. Kundendienstbezogene Argumentationen werden dagegen in hohem Maße alleine durch den EDV-Beauftragten geprägt. Eine Einflußnahme der Unternehmens- und Bereichsleitung erfolgt nur noch sporadisch.

Dagegen zeichnet sich das kundendienstignorante Cluster durch eine insgesamt intensivere Diskussionsbeteiligung aller Personengruppen aus. Gerade bei Kundendienstkäufen ist eine verhältnismäßig stärkere Einflußnahme der Anwender festzustellen.

Zusammengefaßt führt die Analyse der Entscheidungsbeeinflussung zu folgenden Ergebnissen:

- Die Einflußstrukturen PC- und kundendienstbezogener Kaufentscheidungsprozesse sind strukturell vergleichbar,

- die EDV-Beauftragten besetzen generell, aber auch segmentbezogen die führende Fachpromotorenposition in diesen Prozessen,

- die Meinungsbildung vollzieht sich in Gruppenprozessen. Neben dem EDVBeauftragten und der Geschäftsleitung sind Abteilungsleiter und zum Teil auch Anwender daran beteiligt,

- die Diskussion zwischen Geschäftsführern und EDV-Beauftragten ist bei PCKäufen intensiver als bei Kundendienstleistungen,

- im kundendienstskeptischen Segment bestimmen die EDV-Beauftragten in erster Linie alleine die kundendienstpolitische Meinung. 


\subsection{Initiatoren kundendienstbezogener Kaufentscheidungsprozesse}

Als drittes wesentliches Bestimmungsmoment für das kundendienstpolitische Kaufverhalten ist das Initiatiwerhalten der einzelnen Mitarbeitergruppen zu untersuchen. Im Mittelpunkt dieses Aspektes steht die Frage, durch welche Personen PC- und kundendienstbezogene Kaufüberlegungen maßgeblich angeregt werden. 191

Die Untersuchung zeigt, daß vergleichbar zu den Beeinflussungsstrukturen, alle Personengruppen sowohl PC- als auch kundendienstbezogene Kaufanregungen geben. Die Gesamtmarktbetrachtung offenbart darüber hinaus, daß wiederum die EDV-Beauftragten das aktivste Verhalten zeigen und zu derartigen Kaufüberlegungen die stärksten Impulse geben (vgl. Abbildung 34). Ferner ist festzustellen, daß neben Geschäftsführern auch Abteilungsleiter und Anwender PCbezogene Kaufprozesse initiieren. In bezug auf Serviceleistungen reduziert sich jedoch das Engagement dieser drei Gruppen, während das Anregungsverhalten des EDV-Beauftragten weiter zunimmt. Sie stellen mit deutlichen Vorsprung die maßgeblichen Kundendienstkaufinitiatoren ihrer Unternehmen dar.

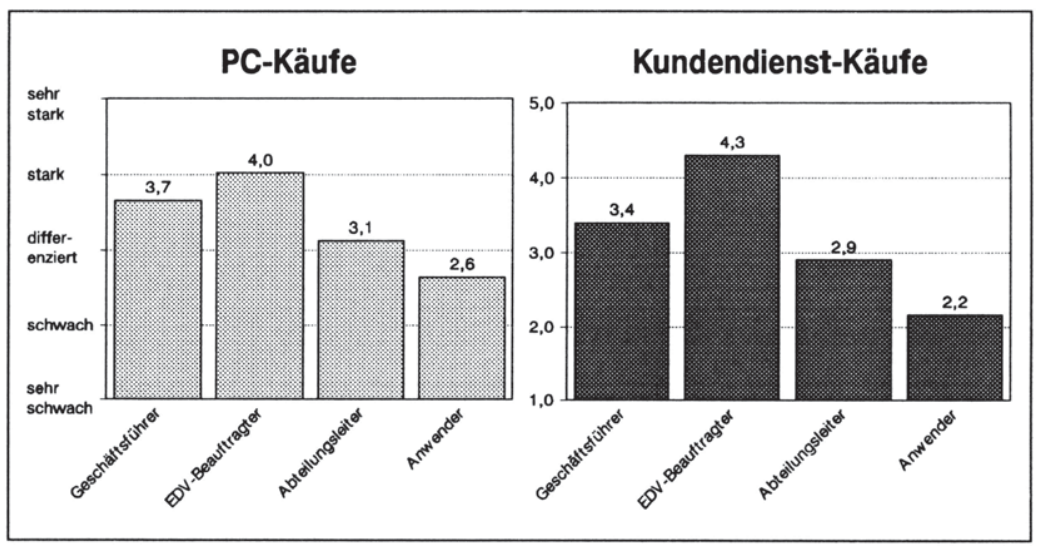

Abbildung 34: Anregungsverhalten zu PC- und kundendienstbezogenen Kaufentscheidungsprozessen

191 Vgl. hierzu Kap. B.3.2. 
Obwohl die Impulse von Geschäftsführern, Abteilungsleitern und Anwendern für Servicekäufe im Vergleich zu PCs schwächer ausgeprägt sind, verändert sich die relative Rangfolge dieser Personen in bezug auf ihr Anregungsverhalten untereinander nicht. Die Zunahme kundendienstbezogener Initiativen seitens der EDV-Beauftragten dokumentiert deren Verantwortung in bezug auf die Einsatzbereitschaft des installierten Bestandes.

Bei differenzierter Betrachtung zeigt sich eine gesamtmarktvergleichbare Initiativsituation im kundendienstunsensiblen Segment. Unterschiede weisen dagegen die anderen Cluster auf (vgl. die Abbildungen 35a und 35b).

Während im Nischensegment das Anregungsverhalten insgesamt relativ schwächer ausgeprägt ist als in anderen Teilmärkten, weisen das Standard-, das kundendienstskeptische und das kundendienstignorante Segment auffallend zentrale Impulsstrukturen auf. Serviceanregungen kommen hier in erster Linie vom EDV-Beauftragten und der Geschäftsleitung. Besonders im letztgenannten Cluster werden PC-Betrieb und Service in hohem Maße durch die Geschäftsführer forciert.

Demgegenüber weist das kundendienstunbetroffene Segment eher anwenderinduzierte Verhaltensstrukturen auf. Besonders in bezug auf Systemkäufe ist ein entsprechendes Engagement festzustellen. Diesbezügliche Anregungen der EDV-Beauftragten sind im Vergleich zu anderen Clustern am schwächsten ausgeprägt. In bezug auf Kundendienstleistungen verändern sie jedoch ihr Verhalten und geben deutlich stärkere Diskussionsanstöße.

Im Ergebnis läßt sich das Anregungsverhalten zu PC- und Kundendienstkäufen damit wie folgt zusammenfassen:

- Anregungen zu beiden Kaufprozessen kommen von allen Mitarbeitergruppen,

- die EDV-Beauftragten besetzen die Initiatorfunktion bei Kundendienstkäufen. Mit Ausnahme des kundendienstignoranten Segments trifft dies auch auf PCProzesse zu (Geschäftsführer),

- stärker als bei den Entscheidungs- und Beeinflussungsstrukturen unterscheidet sich das Bild sowohl in einzelnen Segmenten als auch zwischen der PC- und Kundendienstsituation. 


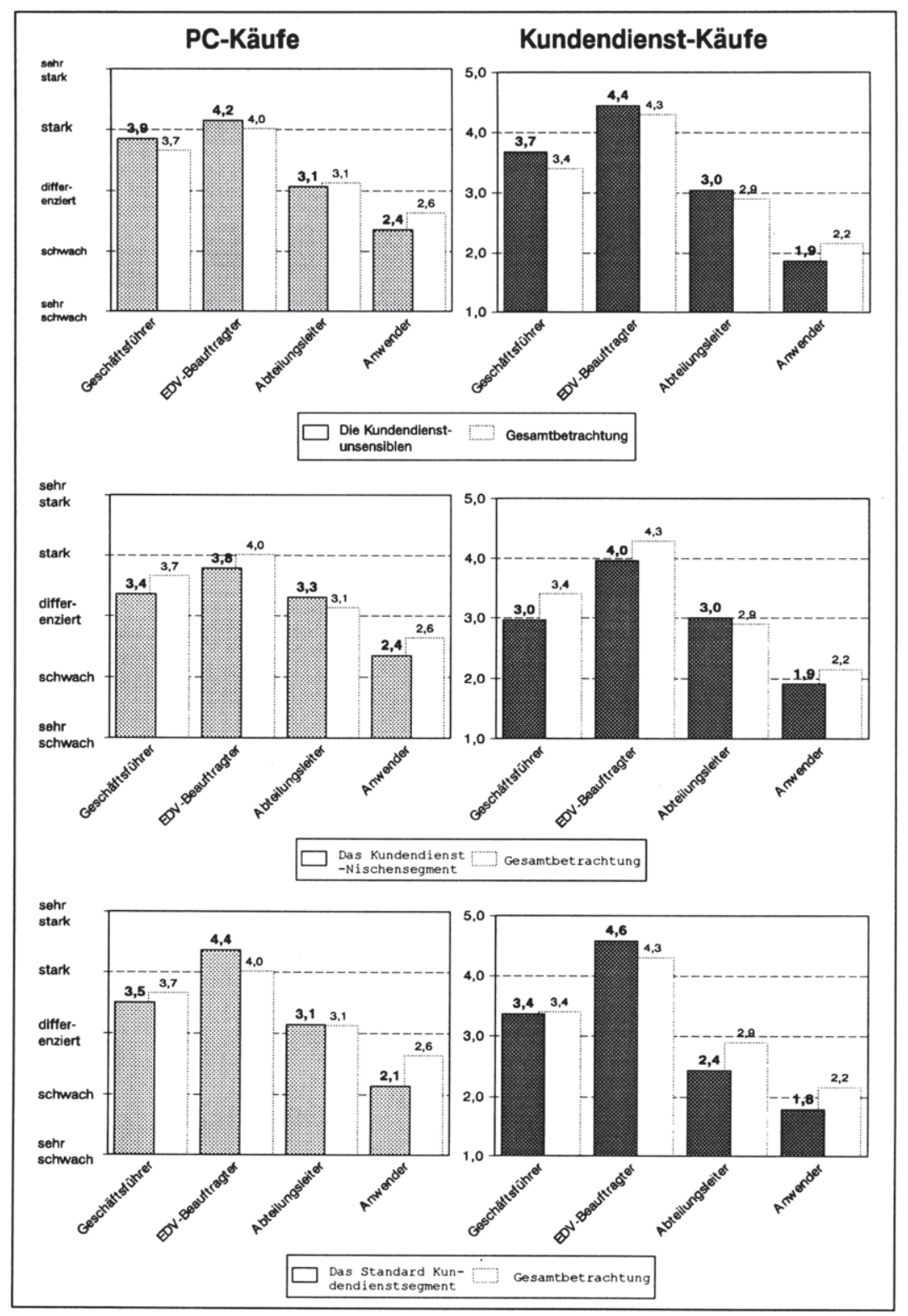

Abbildung 35a: Segmentspezifisches Anregungsverhalten von PC- und kundendienstbezogenen Kaufentscheidungsprozessen (Segmente 1, 2 und 3 ) 


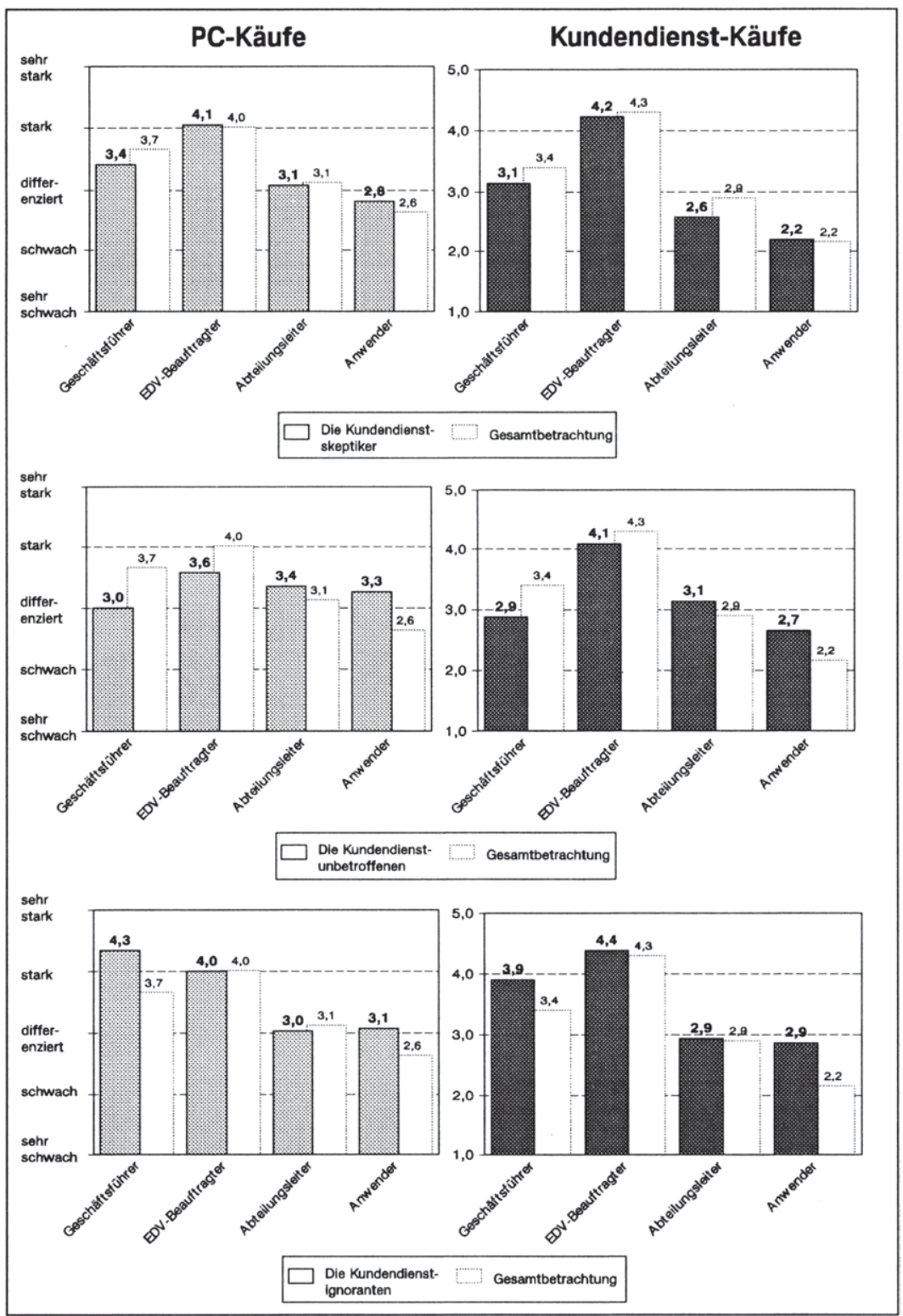

Abbildung 35b: Segmentspezifisches Anregungsverhalten von PC- und kundendienstbezogenen Kaufentscheidungsprozessen (Segmente 4, 5 und 6) 
Die abschließende Gesamtbewertung der PC- und kundendienstbezogenen Kaufprozeßstrukturen erfolgt vor dem Hintergrund der aufgestellten Untersuchungshypothesen.

Die vergleichende Analyse von PC- und kundendienstbezogenen Kaufprozessen hat ergeben, daß sich beide Entscheidungsstrukturen in hohem Maße entsprechen. Dies trifft besonders auf das Entscheidungs- und Meinungsbildungsverhalten der Unternehmen zu. Nennenswerte Unterschiede in einzelnen Segmenten existieren diesbezüglich kaum. Teilweise differente Strukturen offenbart dagegen die Analyse der Kaufprozeßanregungen. Trotzdem kristallisiert sich auch hier eine insgesamt segmentübergreifende Dominanz der EDV-Beauftragten heraus. Hypothese $\mathbf{H}_{\mathbf{M}} \mathbf{1}$ kann damit als bestätigt angesehen werden. Die Strukturen kundendienstbezogener Kaufentscheidungsprozesse sind vergleichbar zu denen von PC-Käufen. Erfahrungen des Hauptleistungsmarketing stellen damit ein geeignetes Synergiepotential für die kundendienstbezogene Marktbearbeitung dar.

Dagegen muß die zweite Untersuchungshypothese $\mathbf{H}_{\mathbf{M}} \mathbf{2}$ abgelehnt werden. Die Untersuchungsergebnisse weisen keine hinreichend strukturellen Differenzen im Entscheidungsverhalten der einzelnen Kundendienstsegmente auf (vgl. Tabelle $6)$.

Zwar lassen sich im Einzelfall bestimmte Eigenarten einzelner Segmenten nachweisen, so etwa im KundendienstNischensegment, im kundendienstskeptischen und im kundendienstignoranten Segment. Hinreichend generalisierbare Charakteristika können jedoch nicht bestimmt werden.

Die relevanten Akzente bei Servicekäufen werden durch die EDV-Beauttragten und die Geschäftsführer gesetzt. Beiden Personen gebūhrt besondere Aưmerksamkeit im Rahmen der Marktbearbeitung. Ein Nachweis über segmentspezifische Machtstrukturen bei kundendienstbezogenen Kaufentscheidungen konnte jedoch nicht im erwartetem Umfang erbracht werden. 


\begin{tabular}{|c|c|c|c|}
\hline Entscheidungseinflüsse & $\begin{array}{c}\text { Machtpromo- } \\
\text { tor(en) }\end{array}$ & $\begin{array}{c}\text { Fachpromo- } \\
\text { tor(en) }\end{array}$ & $\begin{array}{c}\text { Kaufprozeß- } \\
\text { initiatoren }\end{array}$ \\
\hline Gesamtmarkt & GF & EDV-B & EDV-B \\
\hline Die Kundendienstunsensiblen & GF & EDV-B & EDV-B \\
\hline Das Kundendienst-Nischensegment & GF/EDV-B & EDV-B & EDV-B \\
\hline Das Standard-Kundendienstsegment & GF & EDV-B & EDV-B \\
\hline Die Kundendienstskeptischen & GF/EDV-B & EDV-B & EDV-B \\
\hline Die Kundendienstunbetroffenen & GF/EDV-B & EDV-B & EDV-B \\
\hline Die Kundendienstignoranten & GF & EDV-B/GF & EDV-B/GF \\
\hline
\end{tabular}

Tabelle 6: Rollensituation in kundendienstbezogenen Kaufentscheidungsprozessen

\section{Beurteilung der empirischen Segmentierungsergebnisse}

Die Frage nach geeigneten Möglichkeiten zur Segmentierung von Servicemärkten bildet seit geraumer Zeit einen zunehmend wichtigen Diskussionsschwerpunkt in der modernen Kundendienstpolitik. Eine kritische Würdigung des Forschungsstandes zur Marktsegmentierung offenbart jedoch, daß aus konzeptioneller Sicht bestehende Ansätze nur unzureichend den kundendienstspezifischen Anforderungen gerecht werden können. Dies betrifft sowohl die Zusammenstellung und Auswahl geeigneter Kriterien als auch den prozessualen Aufbau der Kundendienstsegmentierung. Darüber hinaus bestehen Defizite hinsichtlich einer empirischen Absicherung der wenigen, bislang vor allem theoriegeleiteten Auseinandersetzungen mit servicebezogenen Segmentierungsproblemen.

Vor diesem Hintergrund wurde in der vorliegenden Arbeit versucht, eine theoretische Aufarbeitung und empirische Fundierung des Untersuchungskomplexes am Beispiel des Marktes für kommerzielle Personal Computer zu leisten. 
Unter Berücksichtigung der vorangestellten Zielsetzung sollen die zentralen Erkenntnisse und Ergebnisse der Untersuchung zusammengefaßt und bewertet werden. Hierzu sind vor allem die eingangs aufgestellten Anforderungen und Bewertungsmaßstäbe zur Segmentierung heranzuziehen. ${ }^{192}$

(1) Die Analyse der Modellvoraussetzungen einer Kundendienstsegmentierung

Das dargestellte Konzept zur Kundendienstsegmentierung baut auf der fundamentalen Annahme auf, im Gegensatz zu produktbezogenen Segmentierungsproblemen des Investitionsgütermarketing, bereits a priori die wesentlichen segmentierungsrelevanten Zielpersonen in Unternehmen bestimmen zu kōnnen. Die EDV-Beauftragten rücken dabei in den Mittelpunkt der Untersuchung.

Es konnte eindeutig nachgewiesen werden, daß einerseits eine solche hauptleistungsverantwortliche Funktion in nahezu allen Unternehmensorganisationen implementiert ist. Darüber hinaus ergab die Analyse, daß die EDV-Beauftragten in bezug auf die betrachteten Technologiezusammenhänge im Vergleich zu anderen Mitarbeitergruppen kompetenzführend sind. Die Vermutung einer sachdominanten Zielperson konnte damit bestätigt werden.

Die hierauf aufbauende Zielvorgabe war es dann, bedürnishomogene Kundendienstsegmente im Markt für kommerzielle Personal Computer zu bilden. Hierzu wurde eine indirekte Verfahrensweise gewählt.

(2) Tragfähigkeit und Qualität der hinzugezogenen Segmentierungskriterien

Zunächst wurden die grundlegenden Dimensionen der servicebezogenen Bedürfnissituation, Abhängigkeitsgrad und Kundenkompetenz gebildet. Die herangezogenen Ausgangsvariablen setzen sich zusammen aus:

- makroanalytischen Variablen (unternehmens-, verwendungs- und hauptleistungsbezogene Merkmale),

192 Vgl. Kap B.1.1. 
- mikroanalytischen Variablen (demographische und psychographische Kriterien einzelner Personengruppen).

Die Bewertung dieser Kriterien für Segmentierungsaufgaben ist bereits in einer Vielzahl von Untersuchungen dargelegt worden. Die entscheidenden Vorteile liegen in der einfachen Meßbarkeit, der unproblematischen Zugänglichkeit sowie einer vorteilhaften Wirtschaftlichkeit. 193

Die Nachteile dieser Ausgangsvariablen sollten mittels einer Projektion auf bedürfnisrelevante Brückenvariablen abgebaut werden. Sowohl die Darstellung des Abhängigkeitsgrads als auch die der Kundenkompetenz konnte mit Hilfe von Faktorenanalysen dabei in geeignete Weise vollzogen werden.

Die Beurteilung beider Konstrukte muß jedoch auch wesentlich vor dem Hintergrund ihrer zeitlichen Stabilität erfolgen. Nur wenn dieser Gesichtpunkt in ausreichendem Maße erfült ist, kann eine solche Marktauf- bzw. -einteilung auch zu hinreichend beständigen Segementierungsergebnissen führen. Dieser Aspekt wurden für beide Schlüsselgrößen weitergehend untersucht.

Personal Computer haben in der jüngeren Vergangenheit breiten Einzug in die betriebliche Datenverarbeitung gehalten und stellen bereits heute eine feste Komponente dar. Gegenwärtig gehören die Einsatzfelder von PCs generell noch nicht zu den wichtigen Aufgabenbereichen in den Unternehmen (Bürowesen, Finanz- und Rechnungswesen), d.h. der Abhängigkeitsgrad ist vielfach noch nicht sehr hoch. 194

Alle Erwartungen und Planungen der Befragten zeigen jedoch eindeutig, daß das Aufgaben- und Einsatzspektrum von PC-Systemen in Zukunft breit ausgebaut wird. Personal Computer sollen zunehmend Aufgaben von mittleren und großen Computern übernehmen. Die Abhängigkeit von PCs und in der Konsequenz auch der Kundendienstbedarf wird deutlich steigen.

So wird in der vorliegenden Untersuchung die zukünftige Bedeutung von PCs in der betrieblichen Datenverarbeitung im Vergleich zu heute wesentlich höher eingeschätzt (vgl. Abbildung 36).

$193 \mathrm{Vgl}$. hierzu auch den umfassenden Literaturüberblick in Kap. B.1.1 dieser Arbeit.

194 Vgl. hierzu auch Abbildung 20 in Kap. 3.11. 


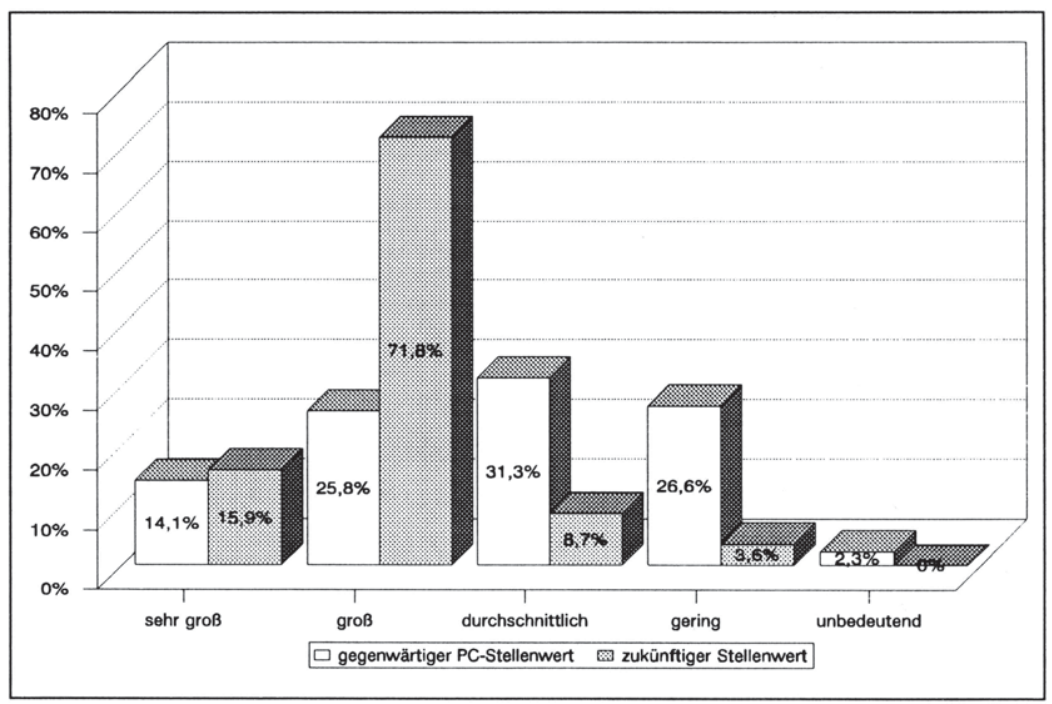

Abbildung 36: Gegenwärtiger und zukünftiger Stellenwert von Personal Computern im Rahmen der unternehmensbezogenen Datenverarbeitung

Ferner konstatiert der überwiegende Teil der Unternehmen $(77,8 \%)$, daß die feste Absicht besteht, das bestehende PC-Wesen im Rahmen der Datenverarbeitung in hohem Maße weiter auszubauen (vgl. Abbildung 37 ).

Eine vergleichbare Entwicklung wird auch die Kundenkompetenz durchlaufen. Ihre Bedeutung für die Kundendienstpolitik ist ebenfalls herausgearbeitet worden. Danach wird eine Kundendienstnachfrage erst dann verstärkt auftreten, wenn die PC-bezogenen Servicebedürnnisse im Unternehmen durch Eigenleistungen nicht mehr ausreichend befriedigt werden können. Kompetenztührend in allen Segmenten sind die EDV-Beauftragten. Der Nutzen von Serviceleistungen ist demzufolge am Maßstab dieses Personenkreises zu messen. ${ }^{195}$

Personal Computer weisen jedoch gegenüber anderen EDV-Systemen einen gravierenden Unterschied auf. Das Anwendungsverständnis dieser Technologie und damit eine gewisse Grundkompetenz ist bei vielen Menschen zunehmend gegeben. Die Untersuchung hat ergeben, daß dies besonders auf den Perso 


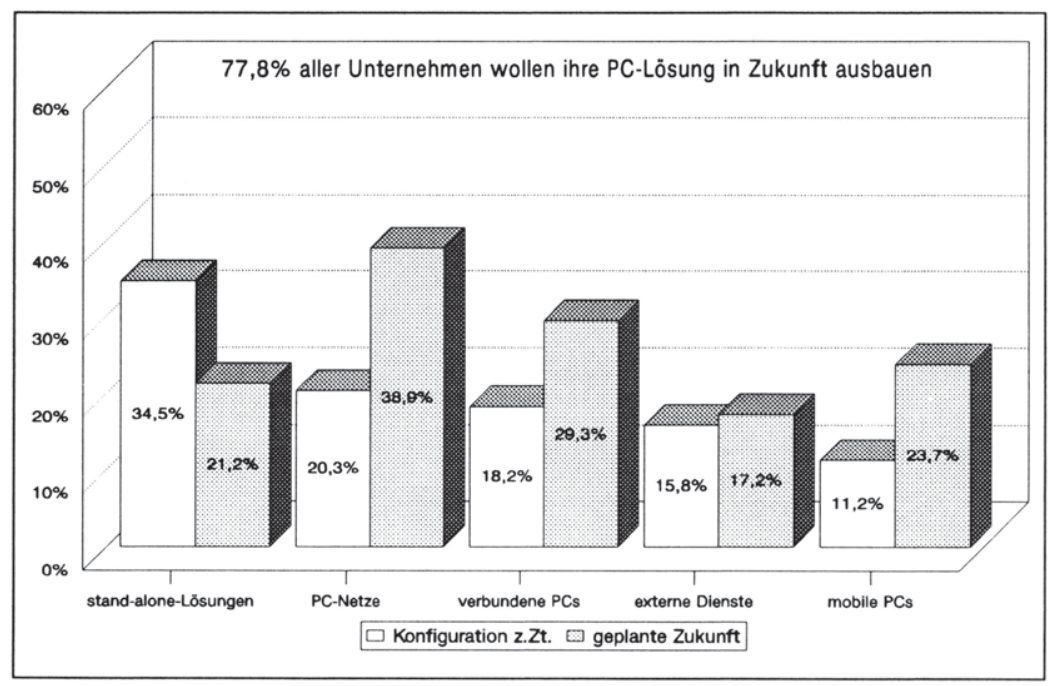

Abbildung 37: Gegenwärtige und geplante PC-Konfigurationen von Unternehmen

nenkreis der EDV-Beauftragten zutrifft. Einer der Hauptgründe hierfür ist die starke Verbreitung dieser Systeme im semiprofessionellen und privaten Bereich. Dabei konnte gezeigt werden, daß bereits zwischen $30 \%$ und $68 \%$ aller EDV-Beauftragten auch im privaten Umfeld PCs benutzen. Dadurch ergibt sich ein beachtenswerter Wissenstransfer "Home-PC" - "Busines-PC". Zudem finden seit Jahren in nahezu allen Ausbildungsbereichen (Schulen, Lehrberufe, akademische Ausbildungsbereiche) Lehrveranstaltungen über PC-Anwendungen statt. PC-bezogene "Berührungsängste" und "Technologiefeindlichkeit" sind somit zum großen Teil ein Generationsphänomen, das zukünftig zugunsten eines ansteigenden Systemverständnisses weiter zurückgehen wird.

Insgesamt gesehen bewirken diese Tendenzen eher einen langfristigen Wandel der bestehenden Kompetenzsituation. Kurz- und mittelfristig erweist sich die Kundenkompetenz ebenso wie auch der Abhängigkeitsgrad als relativ stabile Größe.

Obwohl die beiden Brückenvariablen Abhängigkeitsgrad und Kundenkompetenz langfristig aufgrund der geschilderten Einflüsse an Intensität gewinnen werden, kann damit im Ergebnis der Betrachtungen dennoch festgehalten werden, daß 
sie eine solide Basis zur Segmentierung von Servicemärkten darstellen. Zum einen sind sie zeitlich hinreichend stabil. Zum anderen ist festzustellen, daß auch eine langfristige Intensitätsänderung der Parameter ihre generelle Bedeutung zur Darstellung unternehmensbezogener Kundendienstbedürnnisse nicht mindern kann. So können sich zwar die Gruppentrennungen und -zugehörigkeiten ändern, die Segmentierungskriterien selbst werden jedoch ihre grundsätzliche Geltung behalten.

\section{(3) Qualităt des Segmentierungsergebnisses}

Die Servicebedeutung der beiden Bedürfnisdimensionen, wie auch ihre Eignung Gruppen mit unterschiedlichen Marketingreaktionen und -erwartungen bilden zu können, wurde durch eine Clusteranalyse nachgewiesen. Die gewonnenen sechs-Gruppenlösung weist sowohl hohe inhaltliche Plausibilität wie auch signifikante Trennschärfe in bezug auf die servicebezogenen Bedürfnisvariablen auf.

Die einzelnen Segmente unterscheiden sich in bezug auf die spezifische PC-Implementierung in die unternehmensbezogene Datenverarbeitung. Alle Cluster besitzen darüber hinaus spezifische PC-Kompetenzmuster. Wesentlich für die Ableitung zielgruppengerechter Marktbearbeitungskonzepte ist jedoch vor allem das charakteristische Marketingreaktionsverhalten. Auch diesbezüglich weisen die Segmente typische Strukturen auf. Besondere Schwerpunkte ergeben sich dabei in bezug auf kommunikations- und produktbezogene Aspekte.

Hierzu zählen zum einen gruppenspezifische Einstellungen und Vorurteile gegenüber Kundendienstleistungen (Motive/Barrieren). Auch das Informationsund Weiterbildungsverhalten weist charakteristische Strukturen auf.

Zum anderen ergeben sich Unterschiede in den servicespezifischen Leistungsanforderungen. Wesentliche Gesichtspunkte stellen hierbei u.a. auch die Kundendienstbekanntheit, -erfahrung, -zufriedenheit und -bedeutung dar. Auch besondere distributions- und kontrahierungsbezogene Erwartungen lassen sich z.T. nachweisen.

Das Kaufentscheidungsverhalten der einzelnen Kundendienstsegmente stellt sich dagegen ausgesprochen homogen dar. Geschäftsführer repräsentieren die Rolle der Machtpromotoren, EDV-Beauftragte besetzten die Funktion der Fachpromotoren und Initiatoren. Die Meinungsbildung erfolgt dabei in multipersona- 
len Gruppenprozessen. Diesbezüglich finden auch Abteilungsleiter und Anwender Gehör. Grundsätzliche Abweichungen von dieser Rollensituation können in keinem Segment nachgewiesen werden. Unterschiede ergeben sich allenfalls in bezug auf die Intensität der einzelnen Einwirkung auf verschiedene Entscheidungsprozesse.

Insgesamt kann die vorliegende Untersuchung damit aufgrund ihres Zielerreichungsgrades in hohem Maße überzeugen. Sowohl die konzeptionellen Ausführungen zur Kundendienstsegmentierung als auch die empirischen Ergebnisse stellen einen tragfähigen Beitrag zu einer modernen und differenziert ausgelegten Kundendienstpolitik dar. Tabelle 7 stellt abschließend die zentralen Untersuchungshypothesen zusammenfassend dar.

\begin{tabular}{|l|l|c|}
\hline Bestimmungsgegenstand & \multicolumn{1}{|c|}{ Bestimmungsziel } & $\begin{array}{c}\text { Auswertungs- } \\
\text { ergebnis }\end{array}$ \\
\hline $\begin{array}{l}\text { Spezifische Eigenarten der } \\
\text { Kundendienstsegmentierung }\end{array}$ & $\begin{array}{l}\text { Organisatorische Implementierung einer } \\
\text { spezifischen EDV-Verantwortlichkeit }\end{array}$ & $\begin{array}{c}\text { Hypothese } \\
\text { bestätigt }\end{array}$ \\
\cline { 2 - 3 } & $\begin{array}{l}\text { PC-bezogene Kompetenzführerschaft des } \\
\text { EDV-Beauftragten }\end{array}$ & $\begin{array}{c}\text { Hypothese } \\
\text { bestätigt }\end{array}$ \\
\hline Marktaufteilung & $\begin{array}{l}\text { Operationalisierung der kundendienstspezifi- } \\
\text { schen Bedürnisdimensionen }\end{array}$ & $\begin{array}{c}\text { Hypothese } \\
\text { bestätigt }\end{array}$ \\
\cline { 2 - 3 } & $\begin{array}{l}\text { Identifikation trennscharfer Kundendienst- } \\
\text { segmente im PC-Markt }\end{array}$ & $\begin{array}{c}\text { Hypothese } \\
\text { bestätigt }\end{array}$ \\
\hline Marktbearbeitung & $\begin{array}{l}\text { Homogenität von PC- und kundendienstbe- } \\
\text { zogenen Kaufentscheidungsstrukturen }\end{array}$ & $\begin{array}{c}\text { Hypothese } \\
\text { bestätigt }\end{array}$ \\
\cline { 2 - 3 } & $\begin{array}{l}\text { Unterschiedlichkeit segmentbezogener Kaufent- } \\
\text { scheidungseinflüsse bei Kundendienstkäufen }\end{array}$ & $\begin{array}{l}\text { Hypothese } \\
\text { verworfen }\end{array}$ \\
\hline
\end{tabular}

Tabelle 7: Zusammenfassende Übersicht zur Prüfung der Untersuchungshypothesen 


\section{Implikationen der Untersuchung}

\section{Marktbearbeitungsstrategische Implikationen}

Erfolgversprechende Wettbewerbspositionen bedingen heute mehr denn je klare und eindeutige strategische Positionierungskonzepte. Der im wesentlichen auf Porter zurückgehende Begriff des "stuck-in-the-middle" charakterisiert in diesem Zusammenhang nur zu deutlich die Gefahren einer unzureichenden strategischen Ausrichtung. ${ }^{196}$

Die Marktsegmentierung schafft mit Hilfe der Marktauf- bzw. -einteilung dabei zunächst die notwendigen Voraussetzungen, indem sie dem Strategen einen Überblick über die Strukturen, Gegebenheiten und Anforderungen des betrachteten Gesamtmarktes bereitstellt. Dabei verdeutlicht sich insgesamt das Ausmaß an bestehenden Heterogenitäten. Das Spektrum sinnvoller strategischer Handlungsalternativen wird dokumentiert. ${ }^{197}$

Die meisten heutigen Märkte untergliedern sich in erfolgversprechende Teilsegmente, die untereinander sehr verschiedene Anforderungsstrukturen aufweisen. Eine willkürliche Vernachlässigung solch gruppenspezifischer Besonderheiten führt häufig zu gravierenden Streuungsverlusten und damit letztendlich zum Scheitern anvisierter Zielvorstellungen. Erforderlich, und den Grundprinzipien des Marketing entsprechend, ist vielmehr ein klarer zielgruppengerechter Zuschnitt der Marketingprogramme auf einzelne oder jeweils mehrere Segmente. 198

196 Verfolgt ein Unternehmen keine klare strategische Grundkonzeption, kann der hierbei häufig beobachtbare U-förmige Zusammenhang zwischen ROI und relativem Marktanteil existenzgefährdend sein. Unternehmen sitzen dann "zwischen den Stühlen" und erwirtschaften so vielfach hohe Verluste. Vgl. Porter, M.E., Wettbewerbsstrategie, Frankfurt/M. 1983, S. 83; Meffert, H., Marketing, a.a.O., S. 107.

197 So werden zunächst grundsätzliche Möglichkeiten einer standardisierten oder differenzierten Marktbearbeitung deutlich. Umfang, Größe, Vergleichbarkeit und Potentialabschätzungen des betrachteten Marktes sind hierbei erste relevante Parameter. Vgl. hierzu u.a. Schubert, F., Akzeptanz von Bildschirmtext in Unternehmen und im Markt, Münster 1986, S. $218 \mathrm{ff}$. sowie die dort angegebene Literatur.

$198 \mathrm{Vgl}$. zu den grundsätzlichen Möglichkeiten differenzierter Marktbearbeitungsstrategien insbesondere Meffert, H., Marketing, a.a.O., S. $255 \mathrm{ff}$. 
Die vorliegende Untersuchung von Kundendienstsegmenten im Markt für kommerzielle Personal Computer hat deutliche Gruppenunterschiede aufgezeigt. Die hauptleistungsbezogene Verwendungsrichtung hat sich hierbei als ein zentraler Einflußfaktor herausgestellt. Dieser Zusammenhang ist als weiterer Beleg für die enge Verbundbeziehung zwischen Kundendienst- und Hauptleistungspolitik zu sehen. ${ }^{199}$ Servicestrategien müssen damit besonders auch unter Beachtung der bestehenden PC-Einbindungsformen in einzelnen Teilmärkten entwickelt werden. Zur Auswahl kundendienstpolitischer Marktbearbeitungsstrategien sind deshalb zwei unterschiedliche Betrachtungsperspektiven erforderlich. Zum einen ist die der PC-Konfiguration zugrunde liegende Aufgabenstellung zu bestimmen. Auf dieser Grundlage können die identifizierten Segmente positioniert werden. Zum anderen lassen sich anhand dieser Situation die Bedarfsstrukturen einzelner Cluster ermitteln. Die Ableitung kundendienstpolitischer Normstrategien ist daraufhin möglich.

\subsection{Formen der Integration von Personal Computern in den Prozeß be- trieblicher Datenverarbeitung}

Die vorliegende Untersuchung hat gezeigt, daß rein physische Hauptleistungsunterschiede zwischen den einzelnen Segmenten kaum bestehen. Es können daher keine signifikanten Aussagen über typische Hardware- und Softwarekonfigurationen getroffen werden, z.B. daß Segment i "große" und Segment j "kleine" PC-Systeme einsetzt. Charakteristische Unterschiede ergeben sich aber im Hinblick auf die Lösungsabsicht, die mit dem Einsatz solcher Systeme verbunden ist. Dies führt zu unterschiedlichen PC-Integrationsformen in verschiedenen Unternehmensprozessen. Diesbezüglich weisen alle sechs Gruppen charakteristische Strukturen auf. Die Integration von PCs unterscheidet sich dabei in bezug auf die jeweilige Integrationsbreite und tiefe in Unternehmensprozesse. Eine Positionierung der Segmente vor diesem Hintergrund findet in Abbildung 38 statt. 200

199 Vgl. Kap. A.2.

$200 \mathrm{Vgl}$. hierzu auch Abbildung 11 in Kap. B.2.21. 
Grundsätzlich bestehen vier unterschiedliche Möglichkeiten, Personal Computer in die Prozesse der unternehmensbezogenen Datenverarbeitung einzubinden. Dabei definiert die Integrationsbreite das Ausmaß in wieviel einzelnen Unternehmens-bereichen diese Systeme eingesetzt werden. Im Extremfall besteht eine Durchdringung des gesamten Betriebs. Die Integrationstiefe bestimmt die Bedeutung, die diese Rechner im Rahmen der jeweiligen Aufgabenerfüllung besitzen. Sie kann zum einen taktisch/operativen Charakter haben. Derartige PCs werden als substituierbare Arbeitsmittel betrachtet. Die Aufgabenverrichtung entsprechender Arbeitsplätze ist nicht zwangsläufig mit den Systemen verknüpft. Zum anderen besitzen PCs dann eine strategische Integrationstiefe, wenn hoch relevante und sensible Arbeitsprozesse untrennbar mit diesen Systemen verbunden sind.

Die sich so aufspannende 4-Felder-Matrix zeigt neben der statischen Positionierung einzelner Segmente auch prognostische Hinweise über die weitere Diffusion von PC-Technologie. Derartige Implikationen haben besondere Bedeutung für die Entwicklung kundendienstpolitischer Marktbearbeitungsstrategien.

Plausibel erscheint ein Technologieeinstig im ersten Quadranten201 Die PC-Anwendung hat hier noch vornehmlich Versuchscharakter. Die zentrale Fragestellung ist, ob und wie eine Integration weiter fortgeführt wird, oder ob wieder der Technologieausstieg vollzogen werden soll (stay or exit?). 202

Typische Vertreter des ersten Quadranten sind das kundendienstskeptische und -unbetroffene Segment. Beide weisen noch erhebliche Barrieren und Bedenken auf. Dies gilt besonders in bezug auf die Entscheidungsträger. Im kundendienstskeptischen Segment gibt es jedoch zunehmende Adoptionsimpulse seitens der Anwender und damit ist zukünftig eine weitere PC-Integration wahrscheinlich. Durch die steigende Anwendungserfahrung könnte sich auch die Einstellung zum Kundendienst positiv verändern. Im kundendienstunbetroffenen Segment bestehen derartige Anregungen kaum. Die bislang konsequente Präfe

201 Kaum ein Unternehmen wird seine komplette Datenverarbeitung unmittelbar und vollständig auf eine neue Technologieplattorm stellen. Einarbeitungszeiten, Anlaufstörungen, Anfangsunsicherheiten u.ä. würden zu sprunghaften Produktivitătseinbußen führen.

202 Zur Problematik von "stay-or-exit-Entscheidungen" vgl. auch Meffert, H., Strategische Unternehmensführung und Marketing, Wiesbaden 1988, S. 46. 


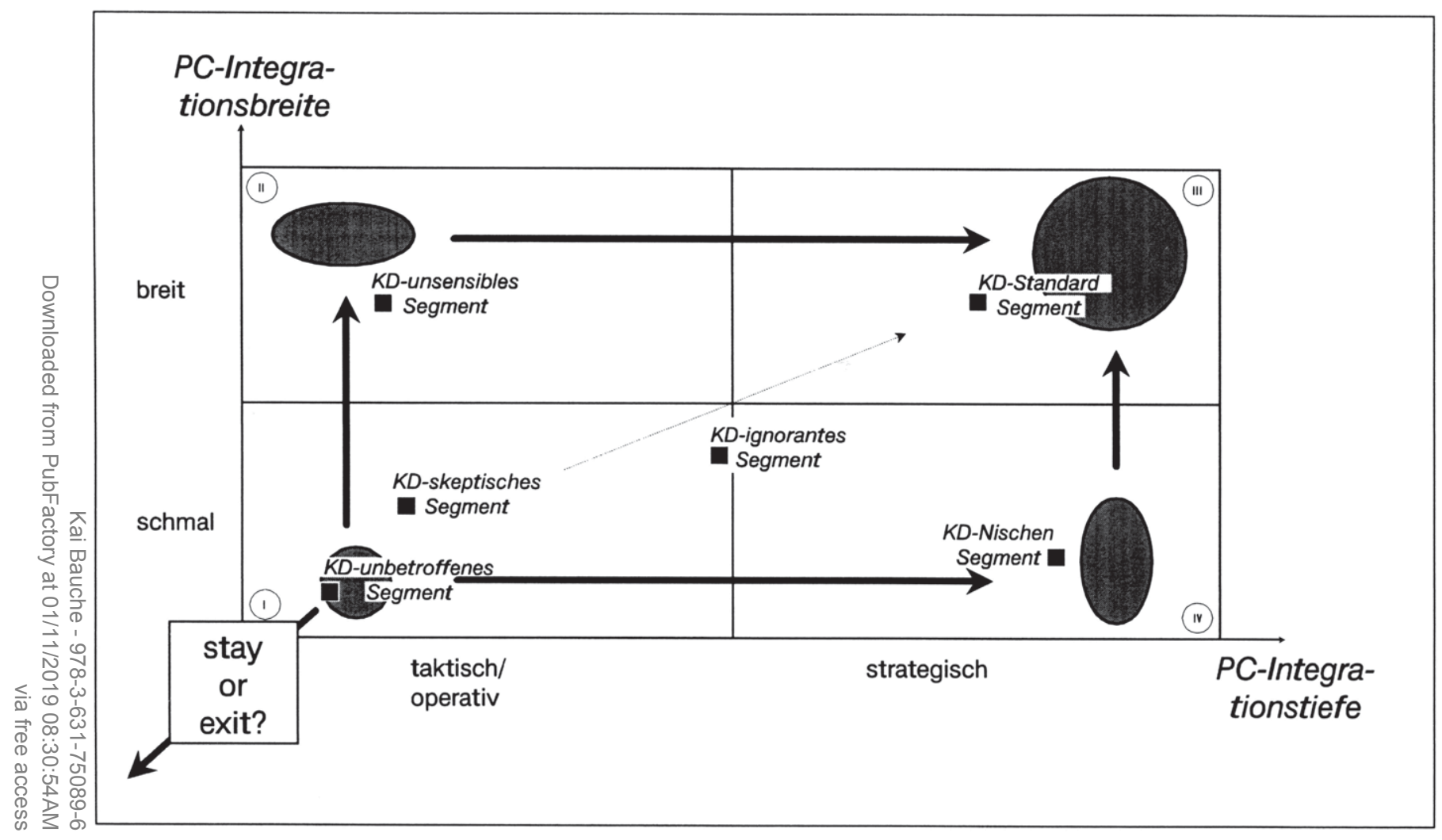

Abbildung 38: Strategien zur Implementierung von Personal Computern in betriebliche Datenverarbeitungsprozesse 
renzneigung dieser Gruppe zu anderen Technologieträgern (Großcomputer, mittlere Systeme) führt in Zukunft u.U. sogar wieder zum PC-Ausstieg.

Im Fall der weiterführenden PC-Integration sind zwei unterschiedliche Diffusionsstrategien denkbar. ${ }^{203}$ Bei breitflächiger Implementierung von PC-Systemen erfolgt ein Aufstieg in den zweiten Quadranten, ohne dabei jedoch gleichzeitig die Lösungstiefe zu erhöhen. Anwender werden dabei zunehmend mit PCs vertraut, was dazu führt, daß breitenwirksame Erfahrungseffekte erzielt werden können. Anwendungen existieren überall im Unternehmen, sind für die jeweilige Aufgabenerfüllung jedoch kaum wichtig. Typischer Vertreter diesbezüglich ist das kundendienstunsensible Segment.

Die andere Alternative den Stellenwert der PC-Lösung auszubauen, ist die tiefere Verknüpfung mit speziellen Unternehmensbereichen. Ein derartiger Ausbau bewirkt eine nahezu vollständige Systemabhängigkeit in einzelnen Ressorts, wogegen alle anderen Datenverarbeitungsaufgaben nach wie vor PC-unabhängig vollzogen werden. Das Kundendienst-Nischensegment weist ein derartiges Erscheinungsbild auf. Mit der Einführung mobiler PC-Lösungen im Außendienstwesen werden wesentliche Degressionseffekte im Kundenkontakt erzielt. Die Datenerfassung vollzieht sich vor Ort und reduziert damit Zeitaufwand und Kosten.

Die höchste Integrationsstufe und damit gleichzeitig auch die ausgeprägteste Abhängigkeitsform von Personal Computern besteht im vierten Quadranten. Im Extremfall werden hierbei sämtliche Datenverarbeitungsaufgaben mit PCs vollzogen. Eine solche Konstellation besteht annähernd im KundendienstStandard Segment. Störungen der PC-bezogenen Datenverarbeitung führen zwangslāufig zu erheblichen Konsequenzen im Unternehmensprozeß.

Der gleichzeitige breiten- und tiefenwirksame Ausbau des PC-Wesens erscheint hingegen bedenklich. Um die datenverarbeitungsbezogenen Betriebsabläufe nicht zu gefährden, erfordert eine solche Handlungsweise während der gesamten Periode des Übergangs einen parallelen Einsatz von Personal Computern und anderen Computersystemen. Die konsequente Verfolgung dieser Strategie

203 Zu Diffusionsaspekten vgl. Rodgers, E.M., Diffusion of Innovations, 3. Auff., New York 1983; Buchner, D., Marketing und Diffusionsforschung, in: Der Marktforscher, 14. Jg., 1970, S. 12; Schulz, D., Kaufentscheidungsprozesse des Konsumenten, Wiesbaden 1972, S. 41 f. 
führt dabei u.U. auch zu einer Verdopplung der Datenverarbeitungskosten. Bis die vollstāndige Umstellung aller Betriebsabläufe erfolgt ist, sind z.T. dieselben Datenbestände auf unterschiedlichen Computersystemen zu pflegen, Mitarbeiter zweigleisig aus- und weiterzubilden, doppelte DV-Betriebskosten hinzunehmen u.a. mehr. Erfolgt die Einstellung des herkömmlichen Computersystems zu früh, d.h. bereits vor dem vollständigen Systemaustausch, ist die Leistungsfähigkeit der betrieblichen Datenverarbeitung in erheblichen Maße gefährdet. Eine solch unklare Technologiepositionierung weißt das kundendienstignorante Segment auf. Obwohl mit PCs bereits nahezu alle wesentlichen kaufmännischen Aufgaben vollzogen werden, ist die zunehmend breite und tiefe Integration dieser Systeme bislang nur Z.T. vollzogen worden. Weitere Computer werden dagegen kaum noch eingesetzt. Die datenverarbeitungsbezogene Konzeption dieser Gruppe ist damit in hohem Maße noch unklar.

\subsection{Entwicklung kundendienstpolitischer Normstrategien}

Der unterschiedliche Lösungscharakter von PC-Systemen hat wesentliche Bedeutung für die Generierung kundendienstpolitischer Marktbearbeitungsstrategien. Implikationen ergeben sich hierbei vor allem in bezug auf die kundendienstbezogene Bedarfsvielfalt und -intensität einzelner Segmente (vgl. Abbildung 39).

Die grundsätzliche Bedarfskonstellation eines Unternehmens wird dabei zunächst bestimmt durch das vorhandene Ausmaß an PC-Kompetenz. Je nachdem, wie umfangreich ein derartiges Eigen-Know how zur Verfügung steht, bestimmen sich die Möglichkeiten, Serviceleistungen unternehmensintern durchzuführen.

Oberhalb dieser eigenen Leistungsfähigkeiten können Servicearbeiten nur noch von einem externen Anbieter erbracht werden. Die individuelle Kompetenzschwelle von Unternehmen stellt demzufolge ein Regulativ der kundendienstbezogenen Nachfragesituation dar. Oberhalb dieser fiktiven Grenze definiert sich der eigentlich bedeutsame Nachfragebereich nach Kundendienstleistungen. 


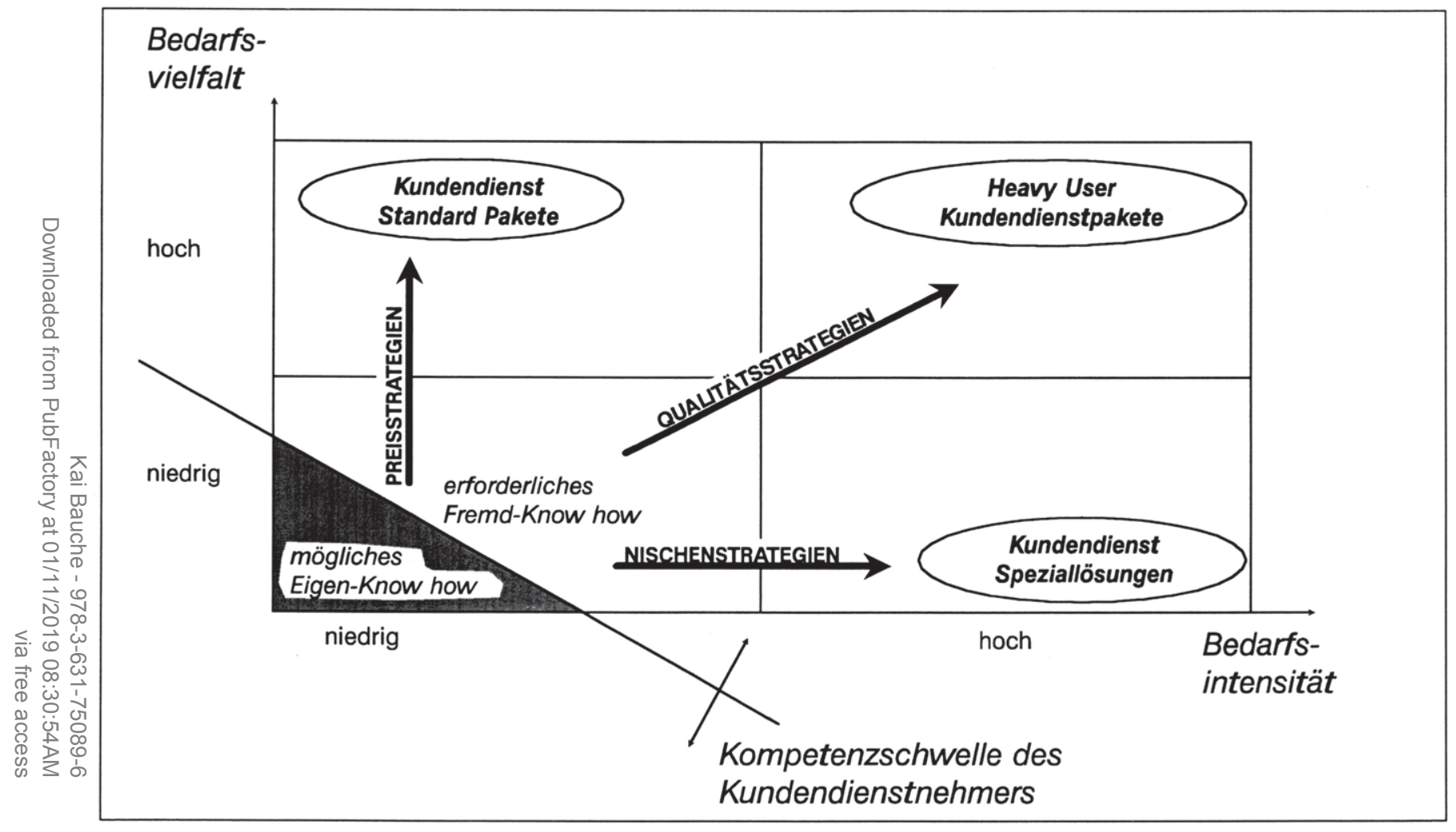

Abbildung 39: Normstrategien der Kundendienstpolitik 
Unter Berücksichtigung der unterschiedlichen Integrationsformen lassen sich dabei drei grundsätzliche strategische Vorgehensweisen ableiten. So bieten sich je nach Umfang der potentiellen Bedartvielfalt und -intensität preis-, qualitätsoder nischenorientierte Marktbearbeitungsstrategien in der Kundendienstpolitik an.

Preisstrategien besitzen besonders in Segmenten mit vielen operativ eingesetzten PCs Bedeutung. Da der Stellenwert dieser Systeme nicht sonderlich hoch ausgeprägt ist, werden die Anforderungen in bezug auf servicebezogene Präventionen und Leistungenen einen verhältnisgerechten Umfang nicht überschreiten dürfen. Ein definiertes Kundendienstvolumen wird zu minimalen Preisen nachgefragt.

Damit rūcken bei Anbietern zwangsläufig Kostengesichtspunkte in den Mittelpunkt der Überlegungen. Wesentlich ist somit, daß Kundendienst im Bedarfsfall möglichst günstig erbracht wird, weniger bedeutend ist dabei die Leistungsgestaltung (Reaktionsgeschwindigkeit und außergewöhnliche Qualität).

Serviceanbieter stehen somit in einem besonderen Preiswettbewerb. Ihre strategischen Bemühungen müssen darauf ausgelegt sein, in diesen Teilmärkten die Preisführerschaft zu erlangen. Diesbezüglich bietet sich die Entwicklung von standardisierten Leistungspaketen an.

Gerade im Bereich der Serviceverträge ergeben sich hierbei vielfältige Gestaltungsmöglichkeiten. Die Entwicklung solcher Kundendienst-Standard-Pakete muß vor allem kostenorientiert erfolgen, d.h. einem effizienten Kostenmamagement kommt eine besondere Bedeutung zu. Überproportional kostensteigernde Leistungen, wie z.B. eine Minimierung der garantierten Reaktionszeiten, Bereitstellung von Ersatzsystemen während der Reparaturzeit u.a. sind zu vermeiden.

Qualitätsstrategien sind in Teilmärkten, deren Datenverabeitung in erster Linie mit Personal Computern betrieben wird, anzuwenden. Die Systeme sind in nahezu allen Unternehmensbereichen verbreitet und von strategischer Bedeutung. Die hieraus resultierende hohe Systemabhängigkeit in diesen Segmenten führt zu einem vielfältigen und intensiv ausgeprägten Kundendienstbedarf. Die Kundendienstanforderungen entsprechen dem hohen Stellenwert der Systeme. Im Mittelpunkt stehen dabei Aspekte der servicebezogenen Leistungsvielfalt und Qualität. 
Kundendienstproduktpolitische Gesichtspunkte bilden für Serviceanbieter damit zwangsläufig den zentralen Schwerpunkt. Das Leistungsmix muß den besonderen Anforderungen der Nachfrager gerecht werden. Kundendienstleister müssen bemūht sein, die Qualitătsführerschaft in diesen Marktsegmenten zu erlangen. Hierzu gehört zum einen die Gestaltung eines umfangreichen und innovativen Kundendienstspektrums. Zum anderen sind die Qualitätsmaßstäbe vergleichsweise hoch festzusetzen. Den Nachfragern reicht es in Störsituationen nicht aus, daß der Kundendienst kommt, sondern daß er schnell und wirksam agiert. Diesbezüglich sind spezielle Leistungspakete zu entwickeln, die den Erfordernissen dieser ausfallsensiblen Intensivnutzer gerecht werden. Die Ausgestaltung solcher Heavy-User-Pakete muß qualitätsorientiert erfolgen. Dabei bieten sich Z.B. Garantien in bezug auf besonders kurze Reaktionszeiten im Rahmen von Serviceverträgen, umfangreiche Back-up-Konzepte, regelmäßige Virenanalyse u.a. an. Preisbezogene Gesichtspunkte stehen nicht im Vordergrund.

Nischenstrategien stellen eine dritte erfolgversprechende Marktbearbeitungsmöglichkeit dar. Anwendung finden sollten solche Konzepte in Märkten mit ganz spezifischen PC-Integrationsformen. Störungen des PC-Einsatzes führen in diesen Segmenten zu Produktivitätsunterbrechungen in wichtigen Unternehmensbereichen. U.U. resultieren hieraus erhebliche Folgewirkungen. 204 Der spezifische Charakter solcher Speziallösungen bedingt entsprechend abgestimmte Kundendienstkonzepte. Im Mittelpunkt steht dabei die adäquate Anpassung an situative Besonderheiten, d.h. Serviceangebote werden speziell auf die Anforderungen derartiger PC-Lösungen zugeschnitten.

Die Entwicklung derartiger Spezialservices erfolgt zumeist für eine verhältnismäBig kleine Zielgruppe. Durch die besondere Abstimmung seiner Leistungen auf die gegebenen Situationen des Hauptleistungsbetreibers, entwickelt der Kundendienstanbieter im Laufe der Zeit jedoch spezifische Lōsungskompetenzen und erlangt so aus Kundensicht letztendlich Wettbewerbsvorteile. Der Kundendienstzuschnitt bzw. seine Individualität stellen das zentrale Element dieser Strategie dar. Im Fall des Kundendienst-Nischensegments bieten sich Z.B. anstelle herkömmlicher Serviceverträge sog. Depotvereinbarungen an. Dabei hält der

204 So kann z.B. der Ausfall einer PC-gesteuerten Fertigungseinhelt zum Stllstand der gesamten Produktion und letztendlich sogar zu einer Unterbrechung des vollständigen Unternehmensprozesses führen. 
Kundendienstanbieter ein zuvor vereinbartes Ersatzrechnervolumen zur ständigen Verfügung des Kunden bereit. Bei PC-Ausfällen können Außendienstmitarbeiter ihren defekten Laptop beim Serviceunternehmen abgeben und erhalten unmittelbar für die Dauer der Entstörung einen Ersatz-PC aus diesem Depot. Es kommt zu keinen Produktivitätsunterbrechungen.

Das marktbearbeitungsstrategische Verhalten gegenüber Segmenten die entweder noch keine klare PC-bezogene Position aufgebaut haben oder grundsätzlich eine weitere PC-Integration noch abwägen, sollte aus Kundendienstsicht vor allem durch kommunikationsbezogene Verhaltensweisen bestimmt sein. Dabei stehen Aufklärungsstrategien im Vordergrund. Das Hauptleistungsmarketing unterstützend, gilt es dabei unstabile Segmente mit nur geringer PC-Abhängigkeit im Systemmarkt und damit auch im Kundendienstmarkt zu halten. Eine enge Abstimmung mit dem PC-Marketing ist hierbei erforderlich. Die gemeinsamen Anstrengungen müssen darauf abzielen:

- die Vorteile einer PC-bezogenen Datenverarbeitung zu vermitteln,

- Einsicht über den Sinn und Nutzen von Kundendienst zu schaffen,

- den PC-Diffusion durch "value added services" zu erhöhen,

- Kontakte zum Kundendienstleister durch servicebezogene "Testangebote" (z.B. preiswerte Systemoptimierung, Virenchecks u.a.) aufzubauen sowie

- durch besondere Einstiegspakete und -konditionen erste Ansätze einer Kundenbindung herzustellen.

Auf eine letztendlich konkrete Ausgestaltung einzelner Marktbearbeitungsmaßnahmen soll im Rahmen dieser Arbeit verzichtet werden. Die Dynamik der wettbewerbsbezogenen Rahmenbedingen sowie der jeweilige verschiedenartige situative Einfluß erscheint zu beträchtlich, um diesbezüglich beständige Konzepte ausgestalten zu können. Vielmehr sollen mit Hilfe dieser Untersuchung grundsätzlich erfolgversprechende Vorgehensweisen dargelegt werden, auf deren Grundlage die Entwicklung konkreter Maßnahmenkonzepte vollzogen werden kann. 


\section{Ansatzpunkte für die weitere Forschung}

Angesichts ihres theoretischen und empirischen Ansatzes entspricht die vorliegende Untersuchung dem Charakter einer Basisuntersuchung. Dabei sind die relevanten Zusammenhänge und Einflußfaktoren einer Segmentierung von Kundendienstleistungen in investiven Märkten am Beispiel des PC-Marktes aufgezeigt worden. In einer abschließenden Würdigung lassen sich folgende Anregungen für weiterführende Forschungsarbeiten auf dem Gebiet der Kundendienstsegmentierung geben:

- Die vorliegende Untersuchung ist aufgrund der Auswahl des Untersuchungsmarktes sehr stark durch die vorherrschende Technologiedynamik geprägt. Die schnellen Innovationszyklen, aber auch die dementsprechend rasche Veralterung verleihen dem PC-Markt einen ganz besonderen Charakter, der sich zwangsläufig auch auf das Kundendienstgeschäft überträgt. Es stellt sich somit die weiterführende Aưgabe vergleichbare Zusammenhänge in technologiebeständigen Märkten zu erforschen, um so den Einfluß der technologischen Dimension sowohl in bezug auf die Markaufteilung vor allem aber in bezug aư die Marktbearbeitung näher bestimmen zu können. 205

- PC-Lösungen stellen dadurch, daß sie aus verschiedenen Teilkomponenten bestehenden (Hardware, Software) eine ganz besondere Hauptleistung dar. Die empirischen Ergebnisse haben gezeigt, daß die kundendienstbezogene Bedürnnisslage im vorliegenden Fall weitestgehend unabhängig vom Leistungsvermögen der einzelnen Systemkomponenten ist. Es bleibt weiterührenden Untersuchungen überlassen, diesen Zusammenhang am Beispiel anderer Produktmärkte zu überprüfen. Dabei ergibt sich möglichenweise ein wesentlich stärkerer Zusammenhang zwischen hauptleistungsbezogenen Leistungskriterien und bestehendem Servicebedarf. Beispielhaft seien Märkte für Industrieaggregate, Lastkraftwagen oder Fluggerăte genannt.

- Eine der wesentlichen Erkenntnisse ist der starke Kompetenz- und somit auch Segmentierungseinfluß semiprofessioneller und vor allem privater An-

205 Zum Einfluß technologiebezogener Aspekte auf das Wettbewerbsverhalten vgl. Meffert, $H$., Die Bedeutung von Konkurrenzstrategien im Marketing, in: Marketing ZFP, Heft 1, 1985, S. $13 \mathrm{ff}$; Brockhoff, K., Technologischer Wandel und Unternehmenspolitik, in: ZfbF, 1984, S. 619 ff.; Zörgiebel, W.W., Technologie in der Wettbewerbsstrategie, Berlin 1983. 
wendungen. Gerade im PC-Markt wird diesbezüglich eine besondere Problematik deutlich. So existieren vielfach die gleichen PC-Lösungen (Hardware, Software) sowohl in Unternehmen als auch in privaten Haushalten. Mehr oder weniger künstlich wird dabei eine vor allem preispolitisch bestimmte Abgrenzung dieser Marktsegmente betrieben. Von besonderem Interesse erscheint hierbei die Frage, ob sich nicht auch segmentübergreifend homogene Kundendienstbedürfnisse und damit völlig neue Servicecluster im gesamten PC-Markt bilden lassen. Die Integration semiprofessioneller und privater Segmente setzt dabei jedoch zusätzlich eine Auseinandersetzung mit den Strukturen des konsumentenbezogenen Kaufverhaltens voraus. 206

- Die Kundenkompetenz stellt ein zentrales Element der Kundendienstsegmentierung dar. In den vorliegenden Ausführungen erfolgte eine grundlegende Aufarbeitung eines nachfragerbezogenen Kompetenzbegriffs im Rahmen der Marketingwissenschaft. Neben der vorliegenden explorativen Vorgehensweise bleibt es Aufgabe weiterer Forschungsarbeiten, dieses Konstrukt, vor allem aber die Wirkungszusammenhänge seiner einzelnen Bestandteile weitergehend zu untersuchen.

- Die Stabilität der beiden Brückenvariablen Abhängigkeitsgrad und Kundenkompetenz wurde anhand von Zukunftserwartungen der befragten Unternehmen überprüft. Dabei wird eine langfristig erwartete Intensitätszunahme beider Größen prognostiziert, die jedoch keinerlei Auswirkungen auf deren grundsätzliche Segmentierungsrelevanz hat. Es bleibt der zukünftigen Forschung vorbehalten, diesen Gesichtpunkt im Rahmen von Zeitreihenuntersuchungen weiterführend zu behandeln. Auf diese Weise können die zeitpunktgestützten Überlegungen bestätigt oder modifiziert werden.

Die Bedeutung von Serviceleistungen wird auch weiterhin in nahezu allen Wirtschaftsbereichen steigen. Gerade deshalb ist und bleibt das Marketing gefordert, sich mit dem Wesen eines modernen Kundendienstmarketing auch zukünftig intensiv auseinanderzusetzten. Aus diesem Grund sollen die angeführten Vorschläge zur weiterführenden Forschung auch nicht als isolierte Fragestellungen im Sinne separater Einzeldisziplinen verstanden werden. Sie sind vielmehr

206 Vgl. hierzu u.a. Müller-Hagedorn, L., Das Konsumentenverhalten, Wiesbaden 1986; KroeberRiel, W., Konsumentenverhalten, 4. Aufl., München 1990. 
vor dem Hintergrund eines integrierten und differenziert ausgerichteten Kundendienstmanagements zu sehen. Daher erhebt sich der abschließende Wunsch, daß sich auch zukünttige Forschungsarbeiten weiterhin kritisch mit den Problemen der Segmentierung von Kundendienstleistungen auseinandersetzen. 
Kai Bauche - 978-3-631-75089-6

Downloaded from PubFactory at 01/11/2019 08:30:54AM

via free access 


\section{Anhang I}

Tabellen der empirischen Untersuchung

Kai Bauche - 978-3-631-75089-6

Downloaded from PubFactory at 01/11/2019 08:30:54AM

via free access 


\section{Verzeichnis des Anhang I:}

Tabelle Al-1: Tabelle der standardisierten Diskriminanzkoeffizienten 161

Tabelle Al-2 Mittelwertunterschiede der clusterbildenden Faktoren (bedürfnisbestimmende Faktoren)

Tabelle Al-3 Mittelwertunterschiede makro- und mikroanalytischer Kriterien. 163

Tabelle Al-4 Mittelwertunterschiede reaktionsbezogener Kriterien 164

Tabelle Al-5 Mittelwertunterschiede reaktionsbezogener Kriterien 165

Tabelle Al-6 Mittelwertunterschiede reaktionsbezogener Kriterien. 166 
Standardized Canonical Discriminant Function Coeffizients

\begin{tabular}{|c|c|c|c|c|c|}
\hline & Func 1 & Func 2 & Func 3 & Func 4 & Func 5 \\
\hline PC-Involvement Geschäftsführer & 0,32312 & $-0,09182$ & 0,36041 & 0,26825 & $-0,20761$ \\
\hline PC-Involvement Abteilungsleiter & 0,17592 & $-0,29344$ & 0,31804 & 0,06716 & 0,18573 \\
\hline PC-Involvement EDV-Beauftragter & 0,71437 & $-0,32959$ & $-0,39345$ & 0,01928 & $-0,22156$ \\
\hline epistemische Anwenderkompetenz & 0,02864 & 0,54443 & 0,19605 & $-0,12145$ & $-0,67028$ \\
\hline heuristische Anwenderkompetenz & 0,05358 & 0,15322 & 0,41748 & $-0,15480$ & 0,16608 \\
\hline freizeitinduzierte Kompetenzeffekte & $-0,42929$ & 0,07928 & $-0,55048$ & $-0,03585$ & $-0,02312$ \\
\hline DV-Erfahrung & $-0,22024$ & $-0,15199$ & 0,25583 & 0,62793 & $-0,34339$ \\
\hline Programmierkenntnis & 0,46231 & 0,50073 & 0,23157 & 0,13470 & 0,00043 \\
\hline Stör- und Ausfallerfahrungen & $-0,05082$ & 0,00542 & 0,20371 & 0,35259 & 0,59777 \\
\hline kaufmānnisches Wesen & 0,46835 & 0,02982 & 0,21628 & $-0,01506$ & 0,09765 \\
\hline Marketingwesen & 0,17836 & $-0,06373$ & $-0,28857$ & 0,30618 & 0,11118 \\
\hline Produktwesen & 0,11648 & 0,61857 & $-0,45349$ & 0,38189 & 0,24599 \\
\hline Außendienstwesen & $-0,27613$ & $-0,30289$ & $-0,00150$ & 0,32106 & $-0,18290$ \\
\hline Ausfallsensibilität & 0,18382 & 0,18175 & $-0,07861$ & 0,02652 & 0,37676 \\
\hline Personalwesen & 0,29473 & $-0,16370$ & $-0,37077$ & $-0,02247$ & 0,14270 \\
\hline
\end{tabular}

Tabelle Al-1: Tabelle der standardisierten Diskriminanzkoeffizienten 


\begin{tabular}{|c|c|c|c|c|c|c|c|c|}
\hline & Kriterien: & Mittelwert & $\begin{array}{c}\text { KD- } \\
\text { Unsensible }\end{array}$ & $\begin{array}{c}\text { KD-Nischen- } \\
\text { segment }\end{array}$ & $\begin{array}{l}\text { Standard KD- } \\
\text { Segment }\end{array}$ & KD-Skeptiker & $\begin{array}{c}\text { KD- } \\
\text { Unbetroffene }\end{array}$ & $\begin{array}{c}\text { KD- } \\
\text { Ignoranten }\end{array}$ \\
\hline & \multicolumn{8}{|l|}{ Abhängigkeitsgrad: } \\
\hline & kaưfmännisches Wesen & 0 & 0,16 & $-0,20$ & 0,29 & $-0,65$ & $-0,49$ & 0,83 \\
\hline & Marketingwesen & 0 & 0,05 & 0,23 & 1,05 & $-0,15$ & $-0,49$ & $-0,22$ \\
\hline \multirow{14}{*}{ 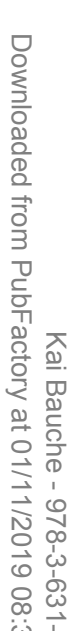 } & Produktwesen & 0 & $-0,37$ & $-0,60$ & 1,71 & 0,48 & $-0,02$ & $-0,03$ \\
\hline & Außendienstwesen & 0 & $-0,05$ & 1,10 & 0,05 & $-0,15$ & $-0,14$ & $-0,48$ \\
\hline & 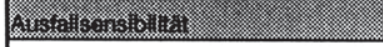 & 0 & 0,30 & $-0,30$ & 0,24 & 0,06 & $-0,74$ & $-0,11$ \\
\hline & Personalwesen & 0 & $-0,04$ & $-0,29$ & 0,47 & $-0,06$ & 0,37 & $-0,13$ \\
\hline & \multicolumn{8}{|l|}{ Kundenkompetenz: } \\
\hline & PC-Involvement Geschăftsführer & 0 & $-0,05$ & 0,49 & 0,46 & $-0,49$ & 0,33 & 0,41 \\
\hline & PC-Involvement Abteilungsleiter & 0 & 0,23 & 0,17 & $-0,01$ & $-0,67$ & $-0,04$ & 0,19 \\
\hline & PC-Involvement EDV-Beauftragter & 0 & 0,43 & $-0,09$ & 0,52 & $-0,07$ & $-0,63$ & 0,14 \\
\hline & epistemische Anwenderkompetenz & 0 & $-0,53$ & $-0,11$ & $-0,07$ & 0,52 & $-0,13$ & 0,88 \\
\hline & heuristische Anwenderkompetenz & 0 & $-0,04$ & $-0,15$ & $-0,11$ & $-0,34$ & 0,34 & 0,44 \\
\hline & freizeltinduzierte Kompetenzeffekte & 0 & 0,01 & $-0,15$ & $-0,17$ & 0,67 & 0,05 & $-0,72$ \\
\hline & DV-Erfahrung & 0 & $-0,38$ & 1,25 & 0,88 & $-0,04$ & 0,00 & $-0,41$ \\
\hline & Programmierkenntnis & 0 & $-0,23$ & $-0,59$ & 1,12 & $-0,10$ & $-0,07$ & 0,69 \\
\hline & Stör- und Ausfallerfahrungen & 0 & 0,07 & $-0,14$ & 0,75 & $-0,50$ & 0,66 & $-0,29$ \\
\hline
\end{tabular}

Tabelle Al-2: Mittelwertunterschiede der clusterbildenden Faktoren (bedürfnisbestimmende Faktoren)

ristit is

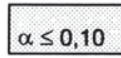

\section{$\alpha \leq 0,05$}




\begin{tabular}{|c|c|c|c|c|c|c|c|c|}
\hline & Kriterien: & Mittelwert & $\begin{array}{c}\text { KD- } \\
\text { Unsensible }\end{array}$ & $\begin{array}{l}\text { KD-Nischen- } \\
\text { segment }\end{array}$ & $\begin{array}{c}\text { Standard KD- } \\
\text { Segment }\end{array}$ & KD-Skeptiker & $\begin{array}{c}\text { KD- } \\
\text { Unbetroffene }\end{array}$ & $\begin{array}{c}\text { KD- } \\
\text { Ignoranten }\end{array}$ \\
\hline & PC-Stellenwert i.R. der Gesamt-EDV & 3,15 & 3,21 & 3,48 & 3,79 & 2,90 & 2,78 & 3,03 \\
\hline \multirow{17}{*}{ 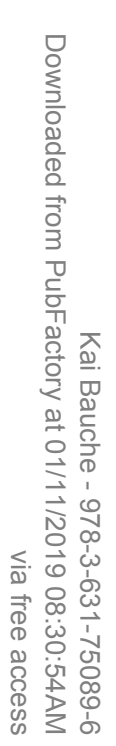 } & PC-Bedeutung f.d. Unternehmenserfolg & 3,98 & 3,96 & 4,30 & 4,57 & 3,65 & 3,788 & 4,07 \\
\hline & zusätzliche EDV-Systeme & $65 \%$ & $54 \%$ & $70 \%$ & $79 \%$ & $84 \%$ & $91 \%$ & $34 \%$ \\
\hline & Anzahl Mitarbeiter & 221 & 203 & 259 & 279 & 234 & 288 & 221 \\
\hline & 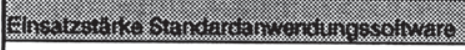 & 3,87 & 3,92 & 4,35 & 3,79 & 3,78 & 3,91 & 3,52 \\
\hline & Einsatzstärke externe Individual-SW & 2,40 & 2,58 & 1,96 & 2,21 & 2,27 & 1,83 & 3,03 \\
\hline & Einsatzstärke interne Indlvidual-sW & 2,23 & 2,24 & 2,13 & 3,14 & 2,30 & 1,61 & 2,28 \\
\hline & Alter des EDV-Beauftragten & 41 & 38 & 41 & 43 & 42 & 45 & 41 \\
\hline & 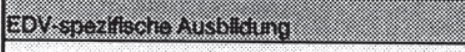 & $33 \%$ & $33 \%$ & $39 \%$ & $21 \%$ & $46 \%$ & $30 \%$ & $21 \%$ \\
\hline & Erfahrung des EDV-Beauftragten (Jahre) & 9 & 7 & 11 & 12 & 10 & 10 & 9 \\
\hline & private PC-Anwendungen & $51 \%$ & $53 \%$ & $57 \%$ & $57 \%$ & $68 \%$ & $30 \%$ & $34 \%$ \\
\hline & Aufgabenvorbereitung durch: & & & & & & & \\
\hline & Heraturstualium & 3,43 & 3,32 & 3,57 & 3,64 & 3,73 & 3,26 & 3,28 \\
\hline & 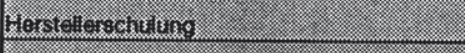 & 2,36 & 2,25 & 1,96 & 2,29 & 2,32 & 2,43 & 3 \\
\hline & 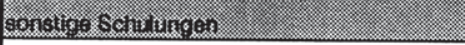 & 3,02 & 2,97 & 3,26 & 3,00 & 3,14 & 2,52 & 3,17 \\
\hline & Fachseminare & 3,22 & 2,92 & 4,17 & 3,93 & 3,14 & 3,13 & 3,03 \\
\hline & 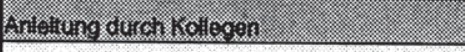 & 2,31 & 2,21 & 2,26 & 2,79 & 2,22 & 2,26 & 2,59 \\
\hline & learning-by-doing & 3,88 & 4,07 & 3,91 & 4,21 & 3,95 & 3,52 & 3,44 \\
\hline
\end{tabular}

Tabelle Al-3 Mittelwertunterschiede makro- und mikroanalytischer Kriterien 


\begin{tabular}{|c|c|c|c|c|c|c|c|c|}
\hline & Kriterien: & Mittelwert & $\begin{array}{r}\text { KD- } \\
\text { Unsensible }\end{array}$ & $\begin{array}{c}\text { KD-Nischen- } \\
\text { segment }\end{array}$ & \begin{tabular}{|c|} 
Standard KD- \\
Segment
\end{tabular} & KD-Skeptiker & $\begin{array}{c}\text { KD- } \\
\text { Unbetroffene }\end{array}$ & $\begin{array}{c}\text { KD- } \\
\text { Ignoranten }\end{array}$ \\
\hline & \multicolumn{8}{|l|}{ Grundservice: } \\
\hline & 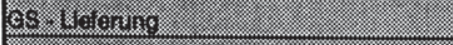 & 4,54 & 4,53 & 4,65 & 4,71 & 4,46 & 4,39 & 4,59 \\
\hline & 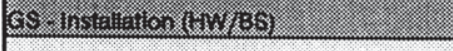 & 3,87 & 3,86 & 3,61 & 3,57 & 3,65 & 4,22 & 4,24 \\
\hline & GS - Installation (AWSW) & 3,17 & 3,33 & 2,65 & 2,43 & 2,78 & 3,61 & 3,69 \\
\hline \multirow{14}{*}{ 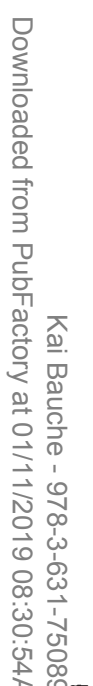 } & GS - Elnwelsung & 3,46 & 3,58 & 2,74 & 2,57 & 3,32 & 3,78 & 4,10 \\
\hline & GS - Schulung & 2,76 & 3,14 & 1,87 & 1,86 & 2,68 & 2,74 & 3,07 \\
\hline & as - Finanderting & 1,55 & 1,53 & 1,35 & 1,14 & 1,54 & 1,70 & 1,83 \\
\hline & GS - Servicevertrag (HW) & 2,87 & 2,69 & 2,83 & 2,29 & 2,46 & 3,70 & 3,48 \\
\hline & GS - Servicevertrag (SW) & 2,60 & 2,81 & 1,87 & 1,71 & 2,19 & 3,17 & 3,14 \\
\hline & as Vesioherting: & 1,87 & 1,89 & 1,70 & 2,00 & 1,65 & 2,04 & 2,03 \\
\hline & GS - Gewährleistungsvereinbarungen & 3,16 & 2,97 & 3,26 & 3,43 & 3,70 & 3,52 & 2,45 \\
\hline & \multicolumn{8}{|l|}{ Servicemotive: } \\
\hline & 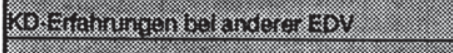 & 2,79 & 2,86 & 2,83 & 3,07 & 2,65 & 2,78 & 2,66 \\
\hline & 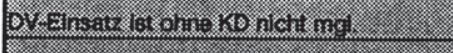 & 3,34 & 3,39 & 3,43 & 3,57 & 3,00 & 3,35 & 3,45 \\
\hline & 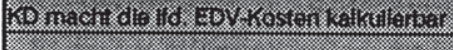 & 2,45 & 2,38 & 2,52 & 2,64 & 2,38 & 2,48 & 2,55 \\
\hline & 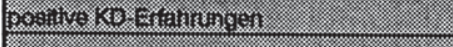 & 2,86 & 2,81 & 2,91 & 3,00 & 3,00 & 2,78 & 2,79 \\
\hline & 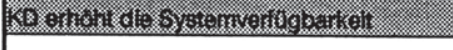 & 3,50 & 3,56 & 3,74 & 3,86 & 3,14 & 3,48 & 3,45 \\
\hline & \multicolumn{8}{|l|}{$\ldots$} \\
\hline
\end{tabular}




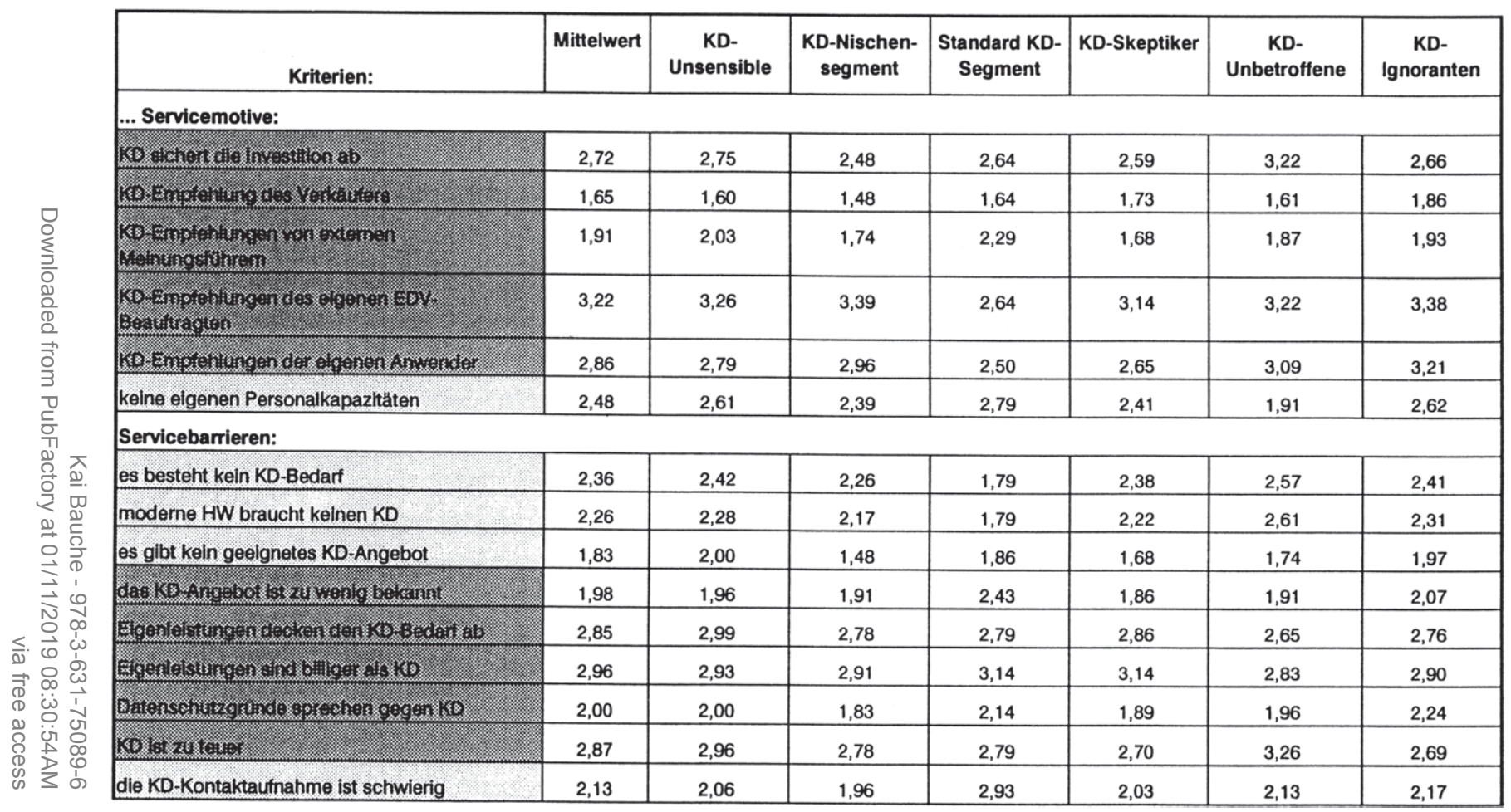

Tabelle Al-5: Mittelwertunterschiede reaktionsbezogener Kriterien 


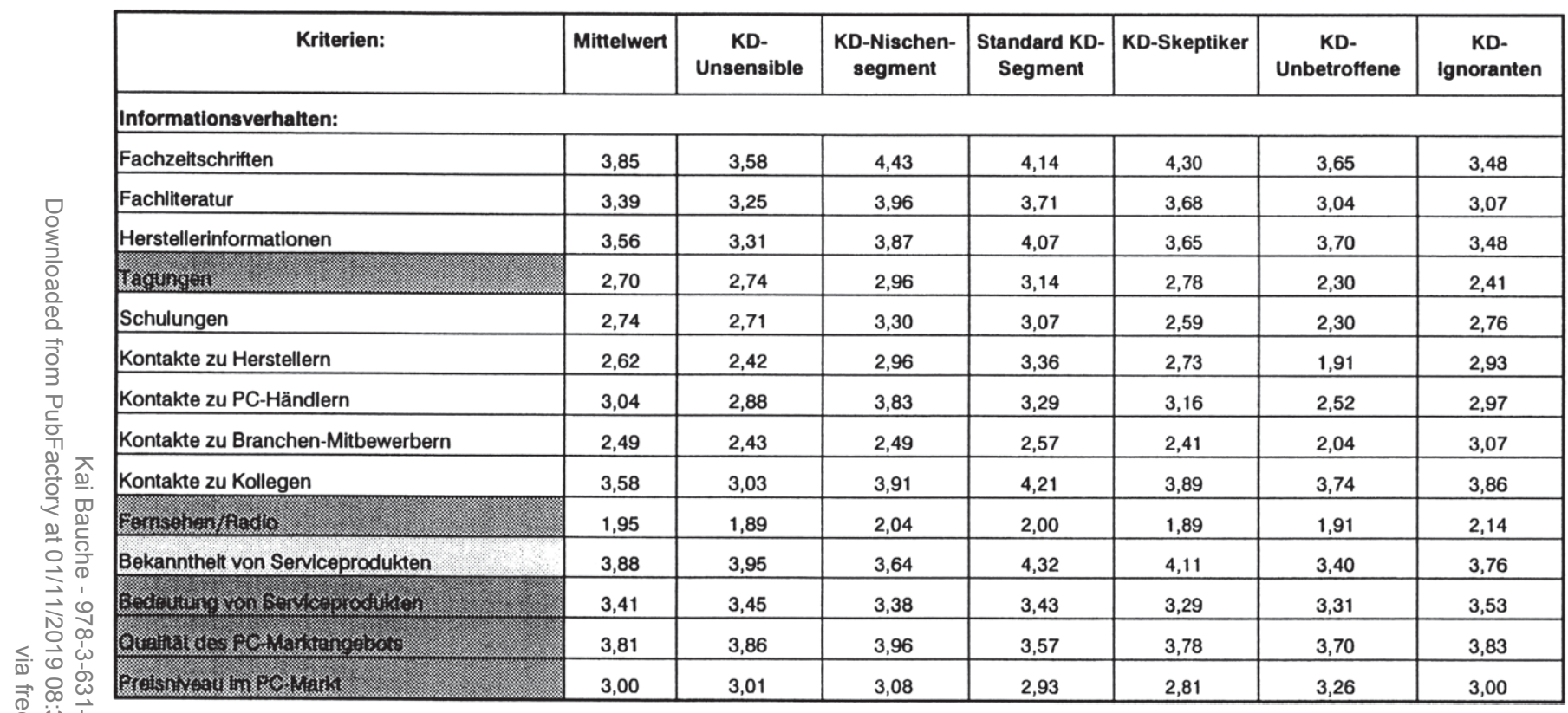


Fragebogen des Geschättsführers

Fragebogen des EDV-Beauftragten 177 


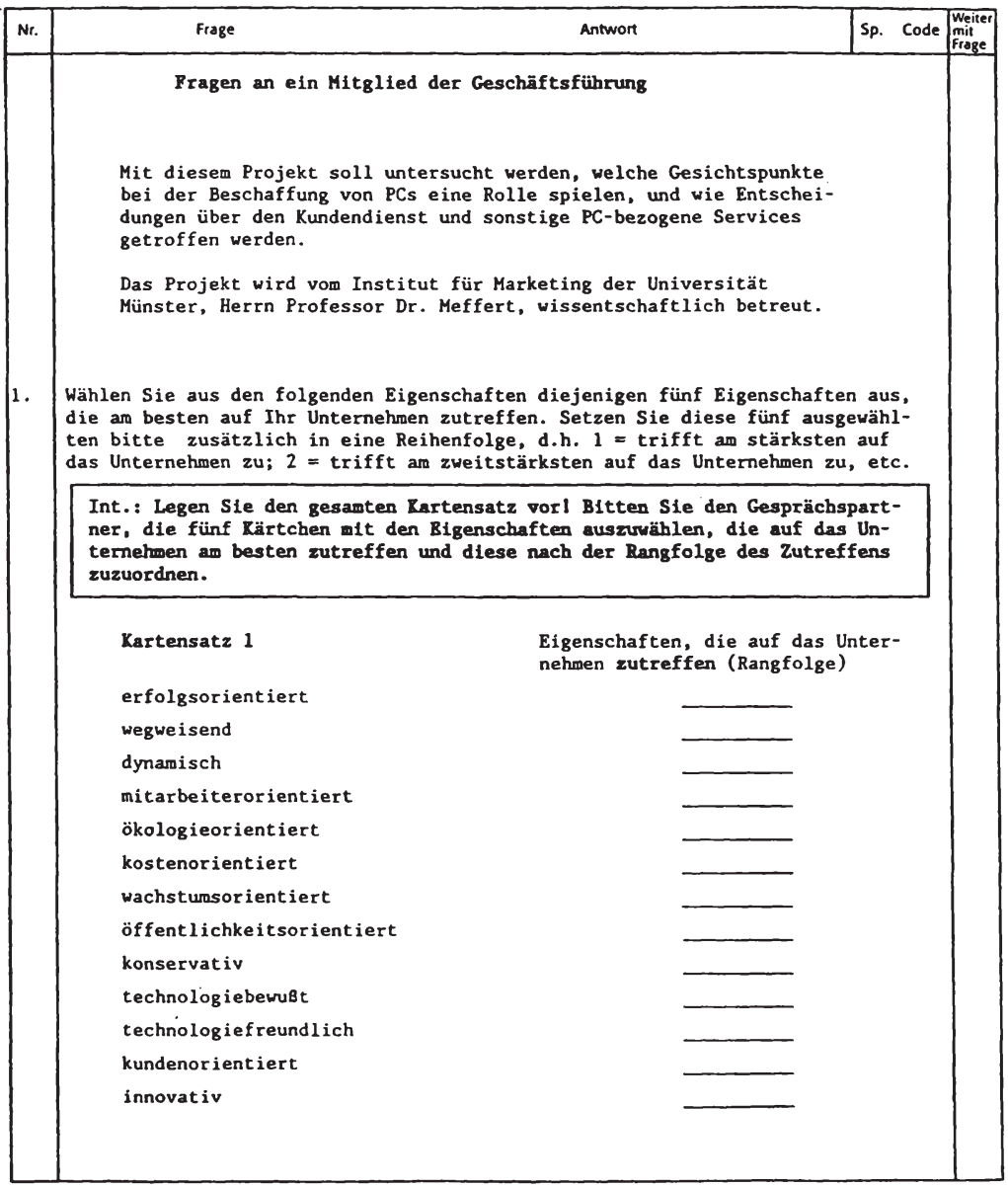


$91-2529 / v g-$ Juni 1991

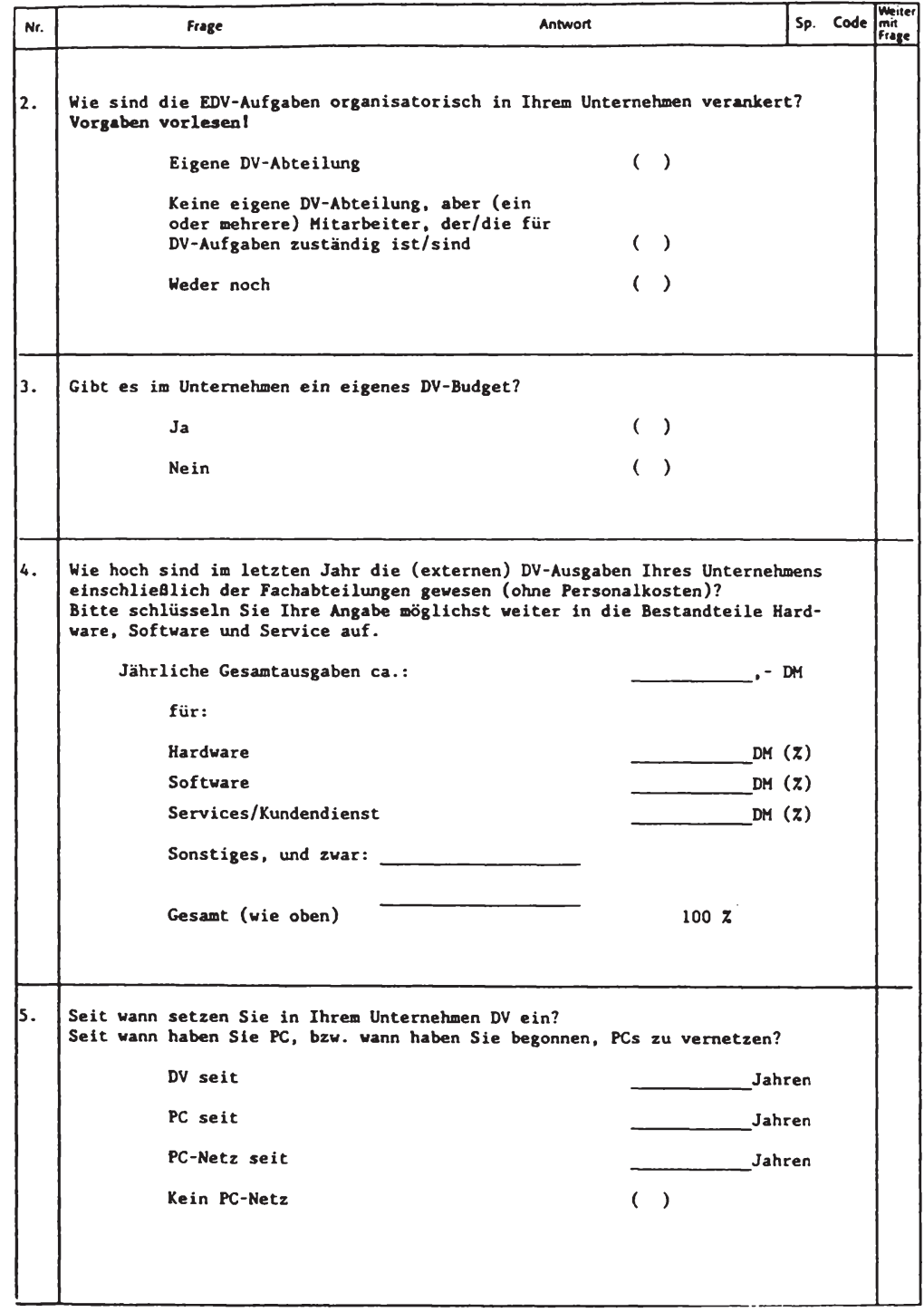


$91-2529 / v g-J u n i 1991$

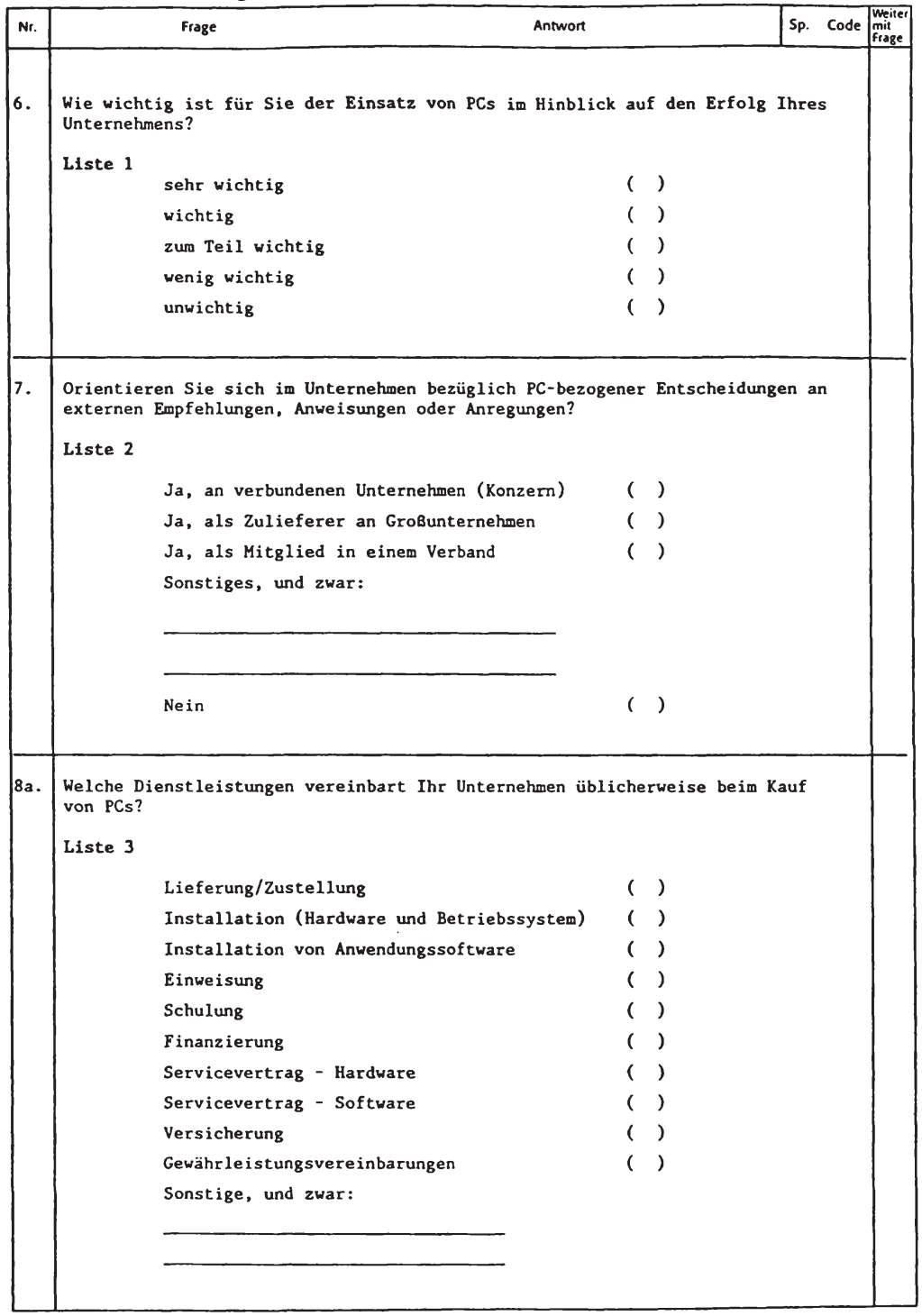


91-2529/vg - Juni 1991

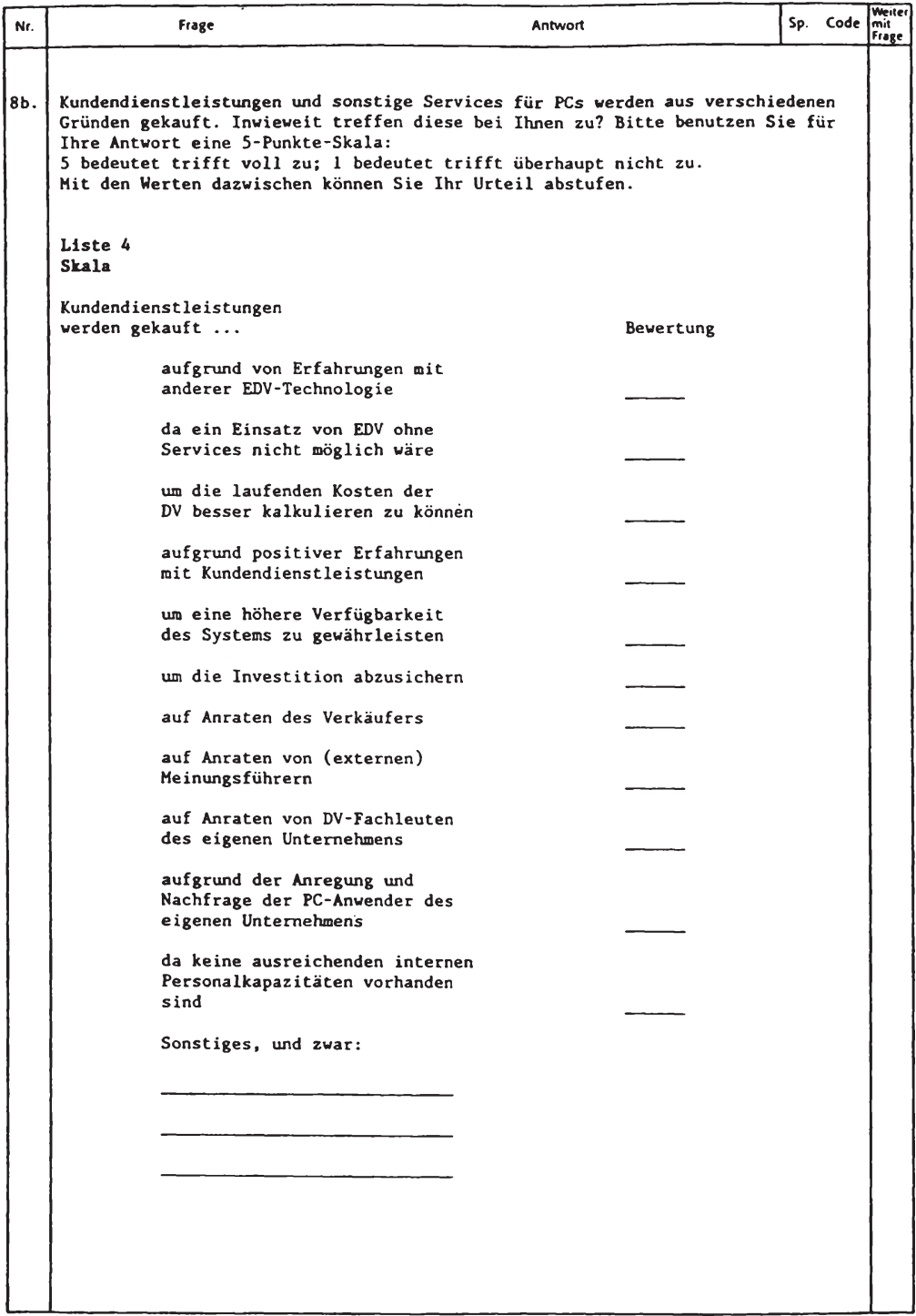


$91-2529 / v g-J u n i 1991$

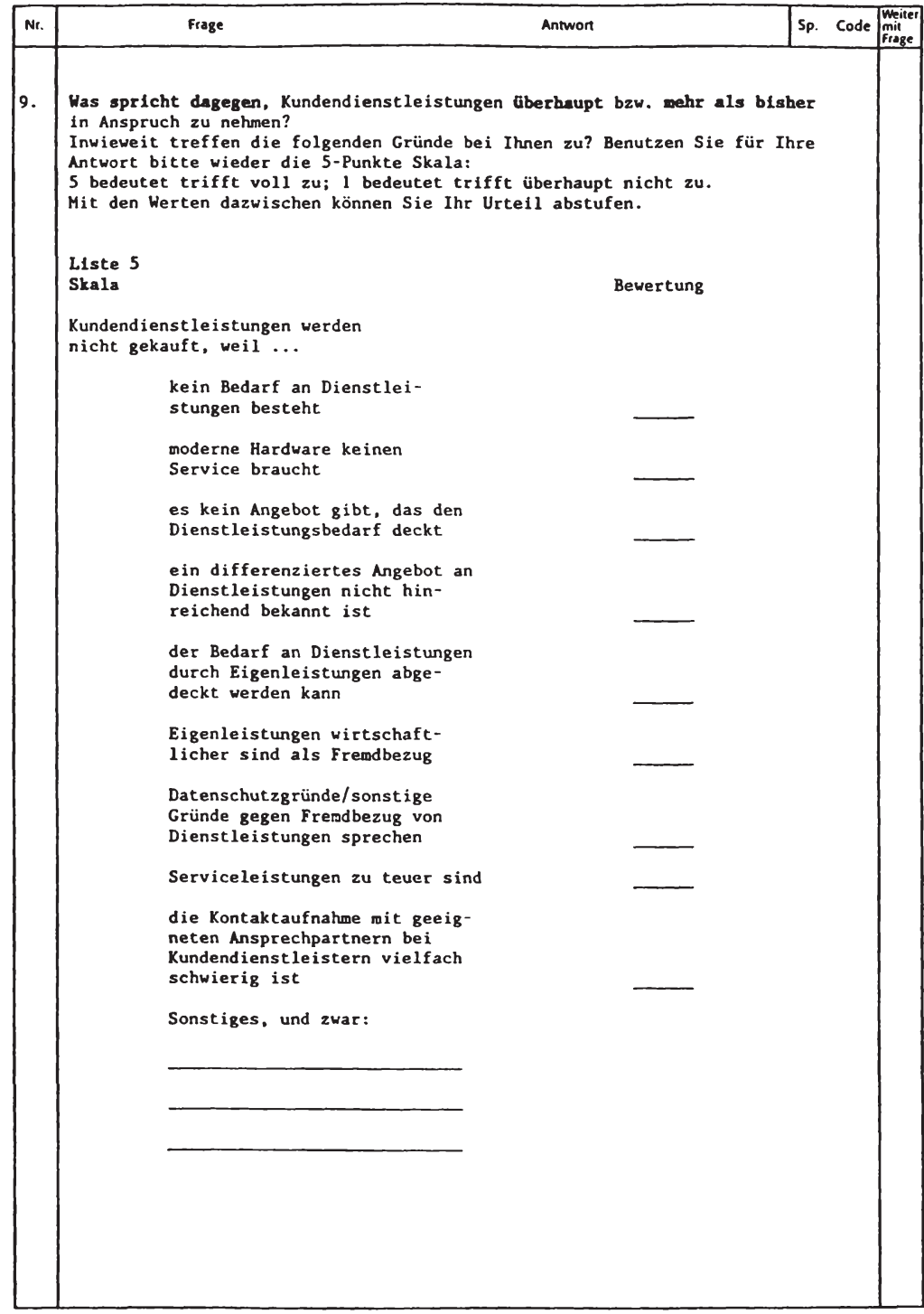


$91-2529 / v g-J u n i 1991$

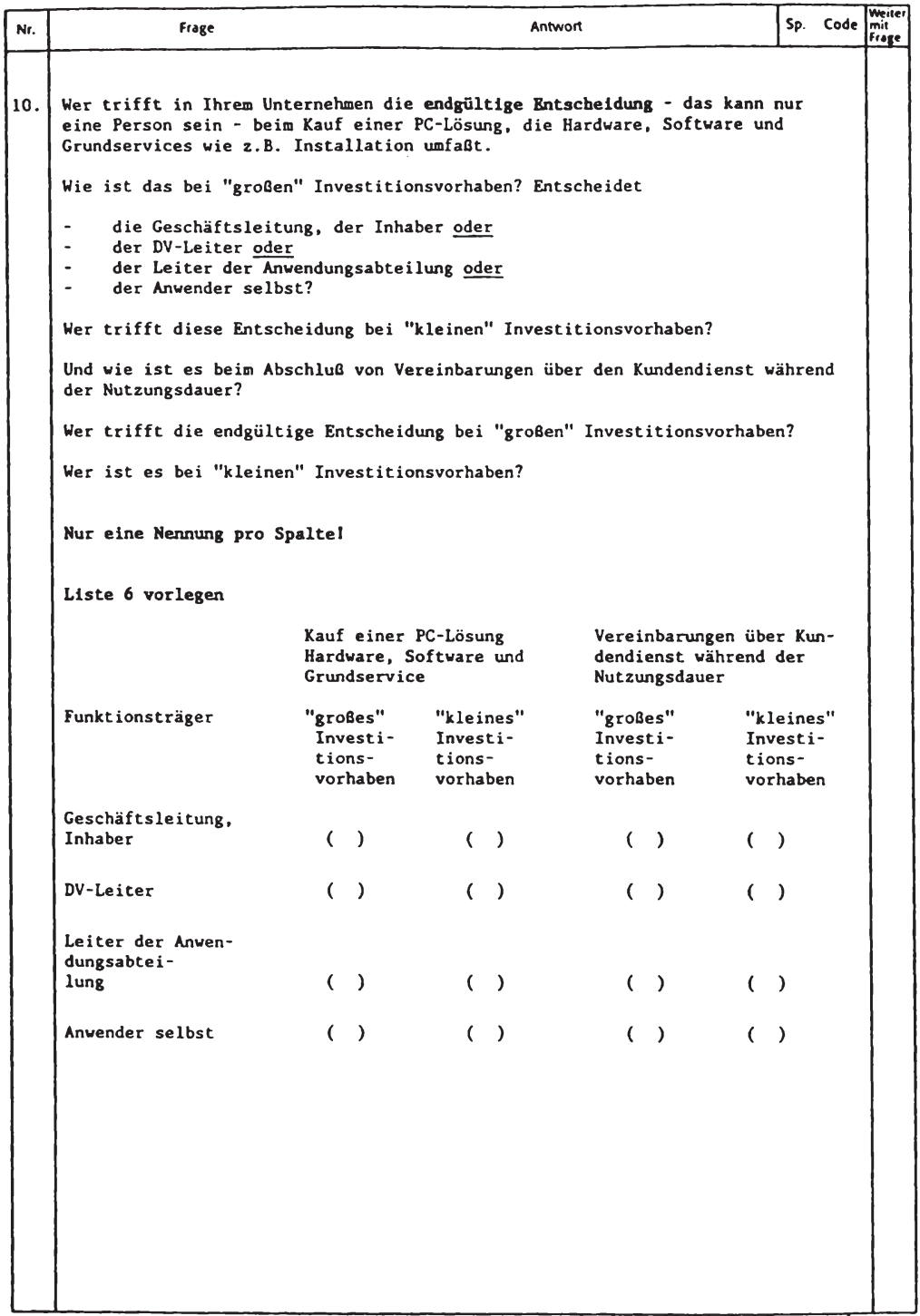


$91-2529 / v g-$ Juni 1991

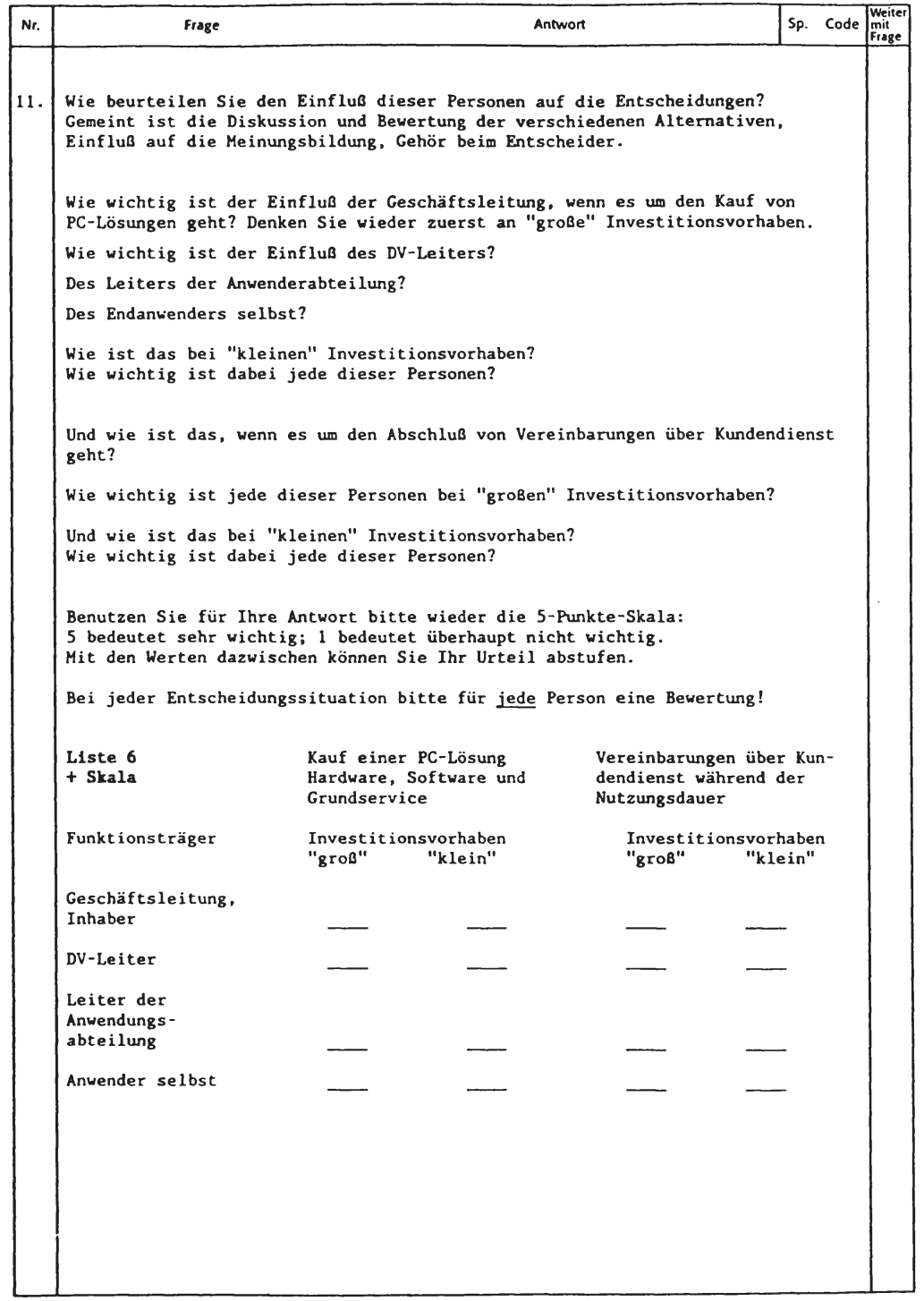


$91-2529 / \mathrm{vg}$ - Juni 1991

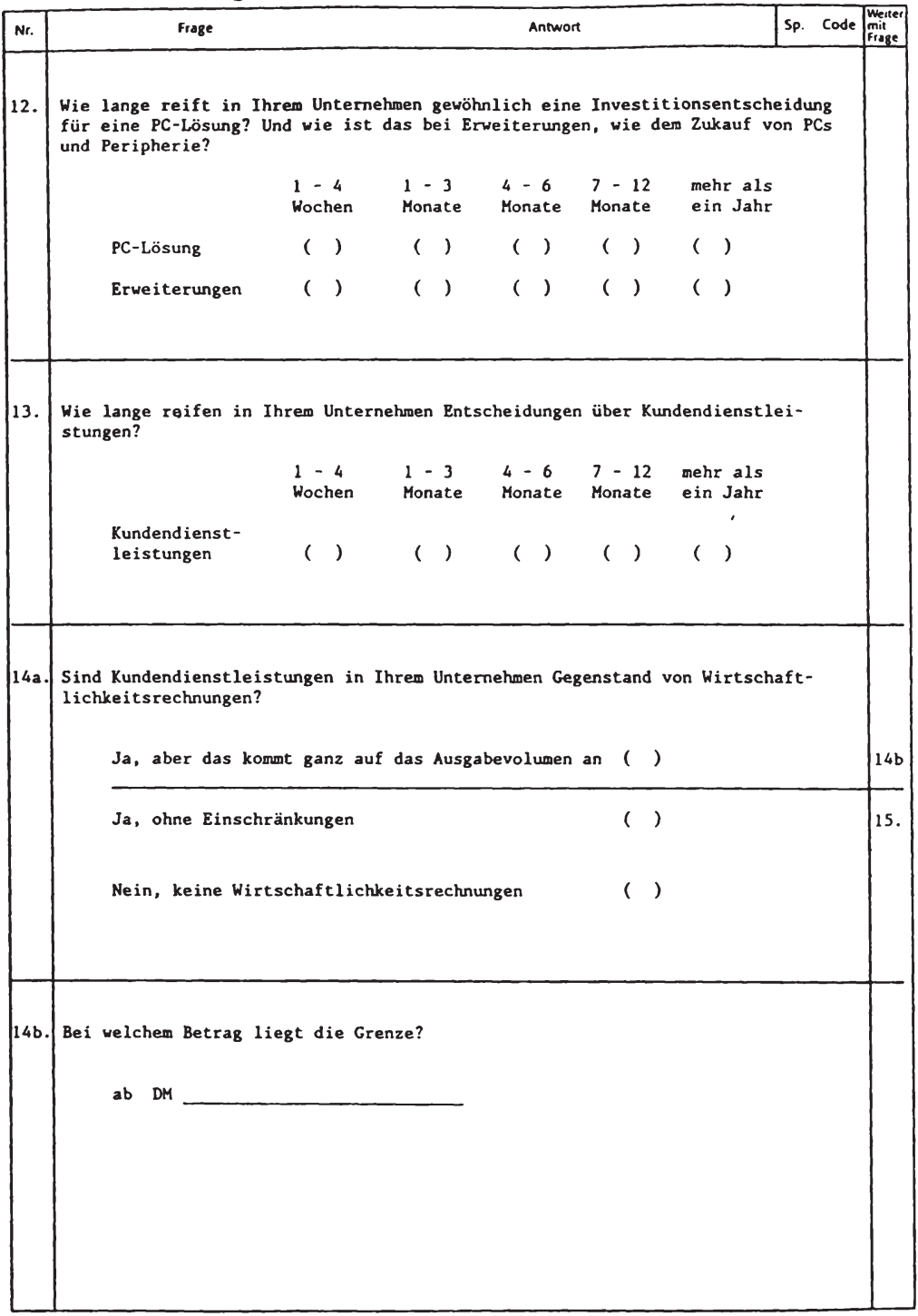


$91-2529 / v g$ - Juni 1991

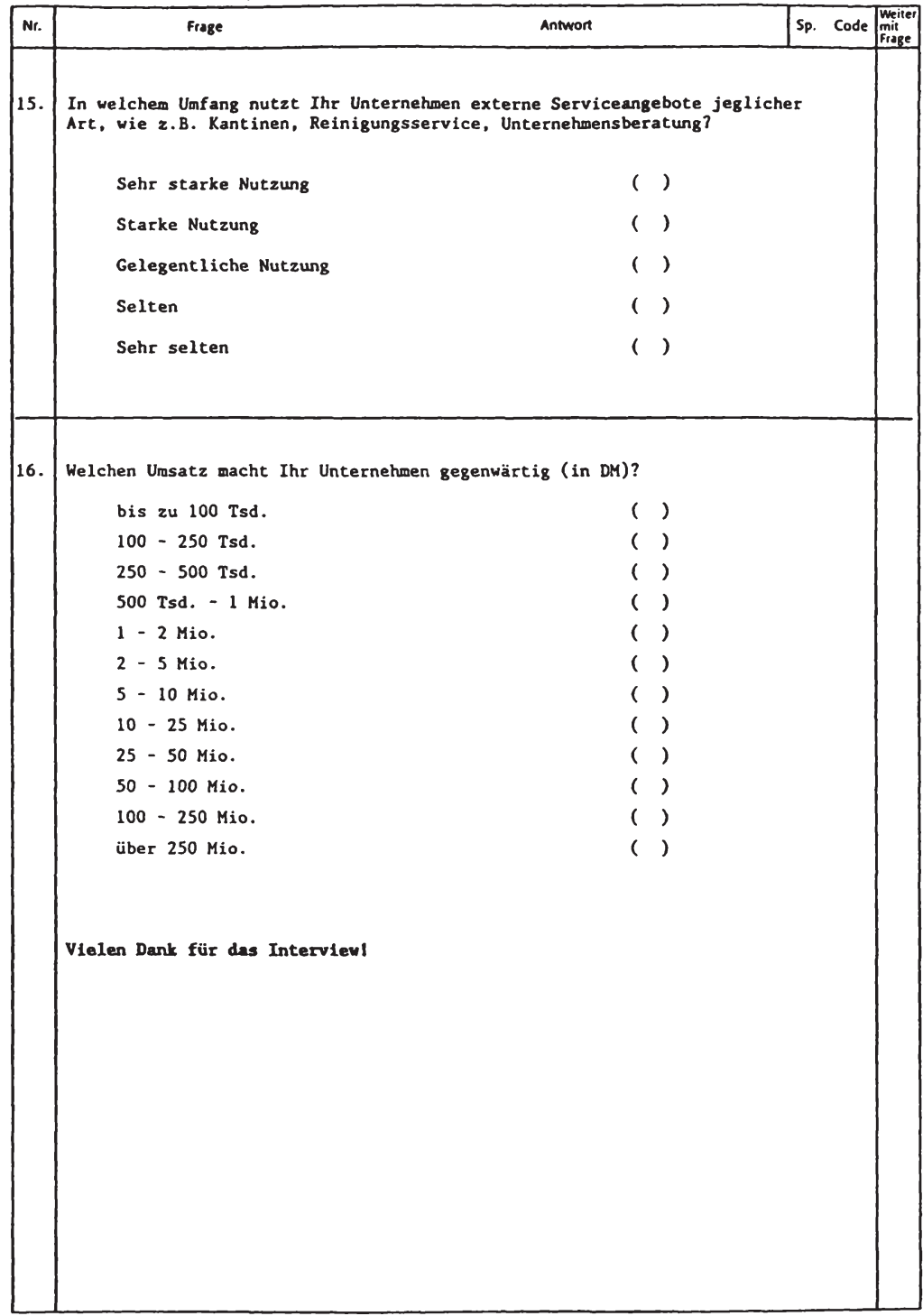




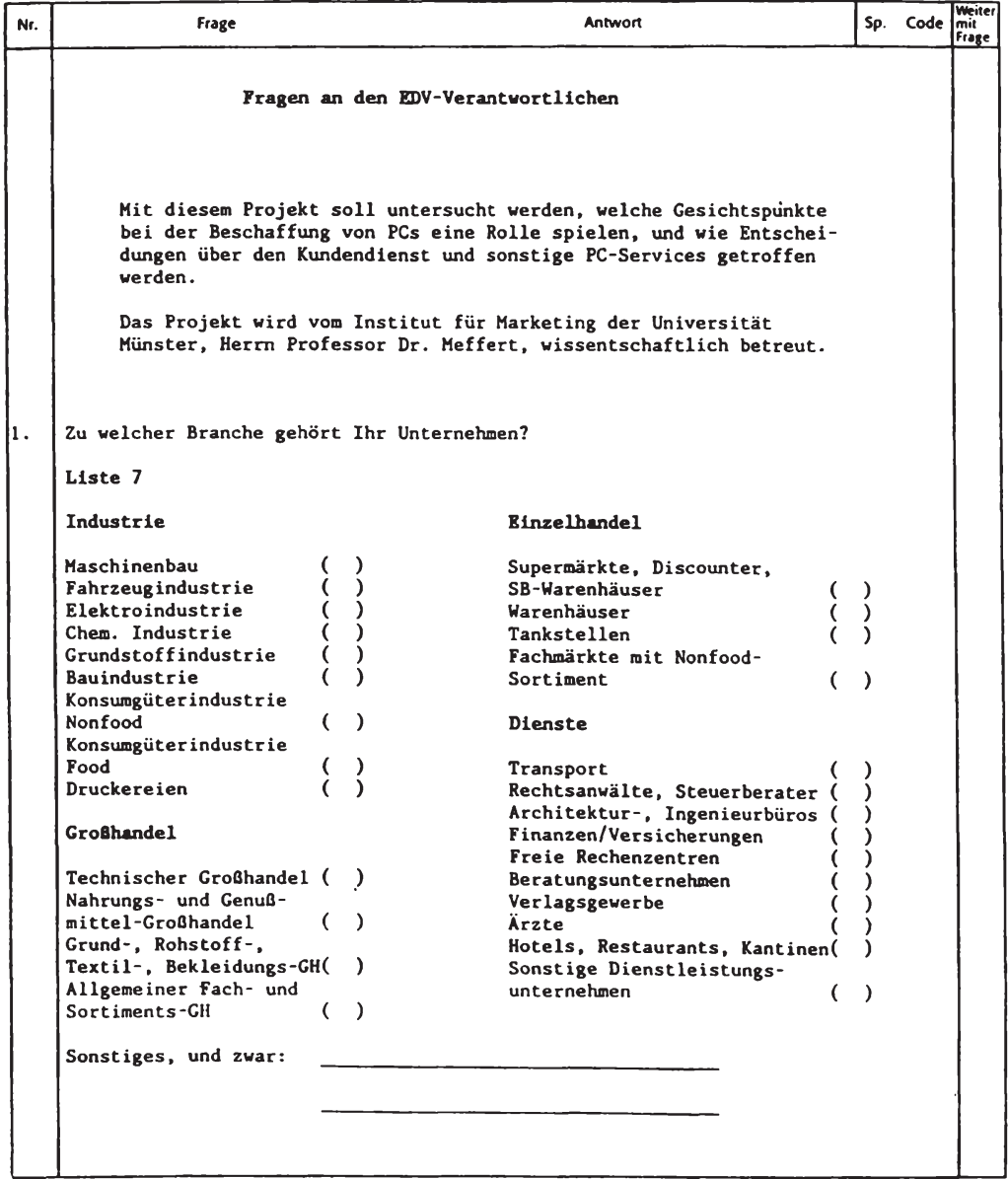


$91-2529 / v g$ - Juni 1991

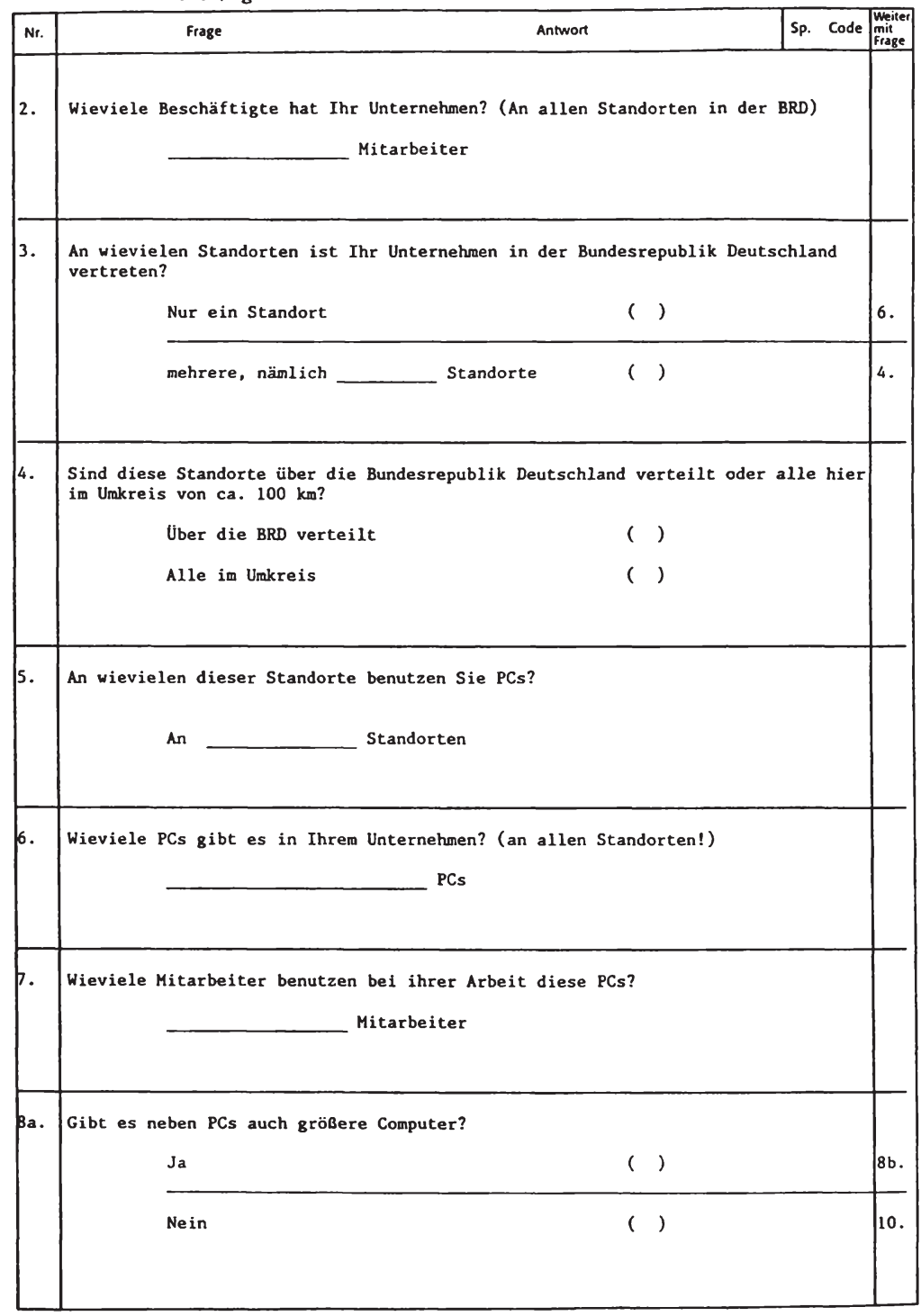


$91-2529 / v g$ - Juni 1991

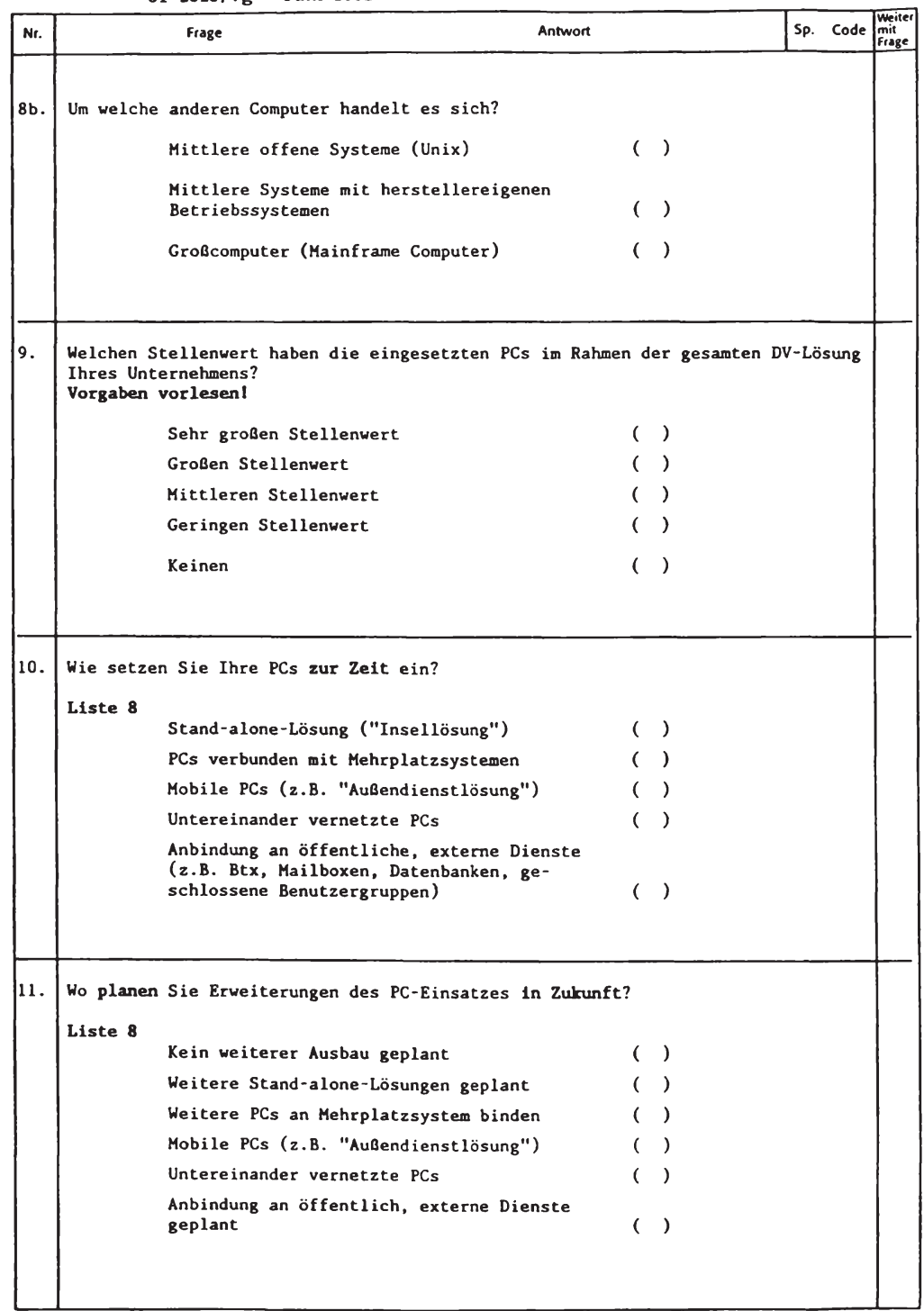


$91-2529 / v g$ - Juni 1991

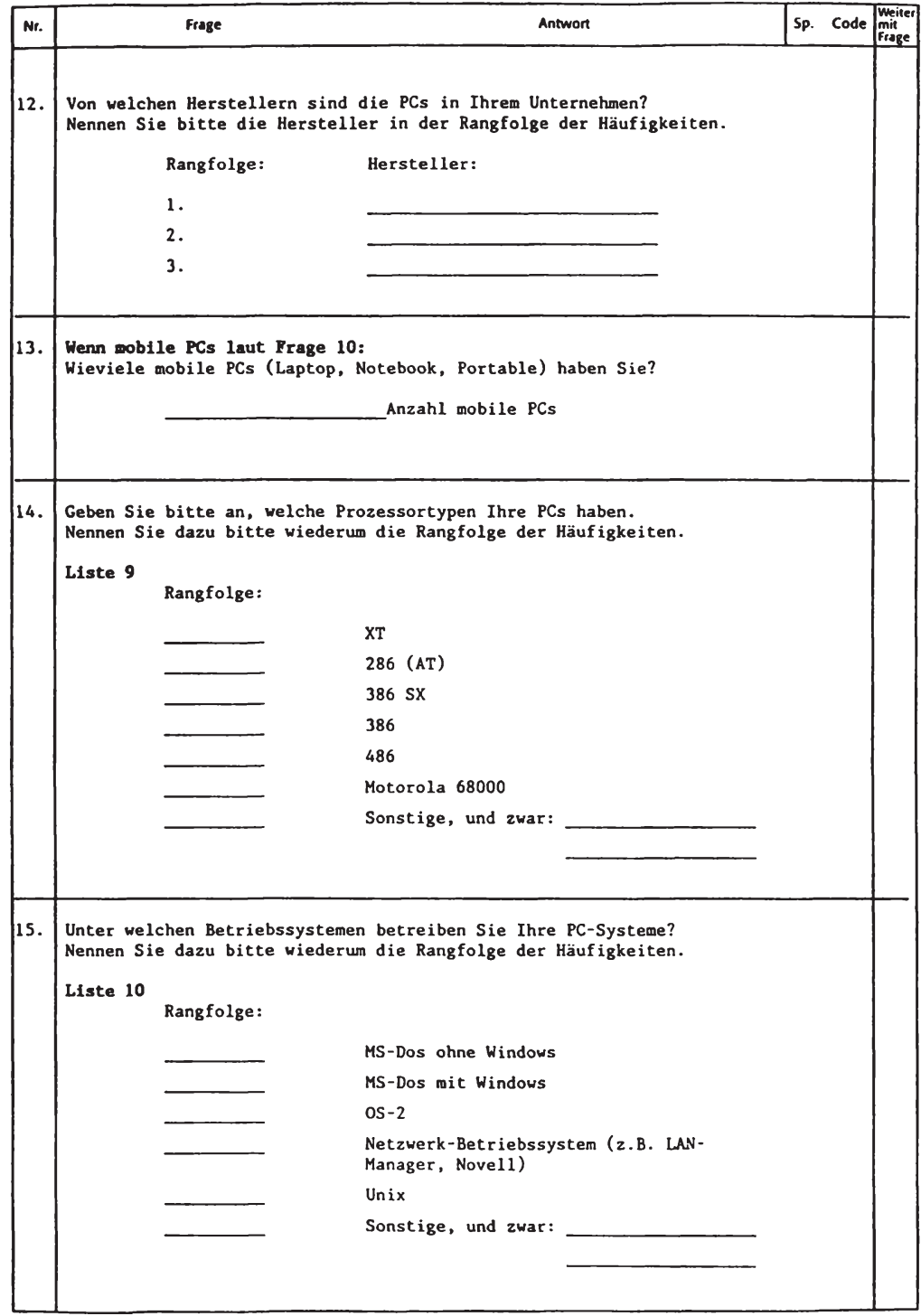


$91-2529 / \mathrm{vg}$ - Juni 1991

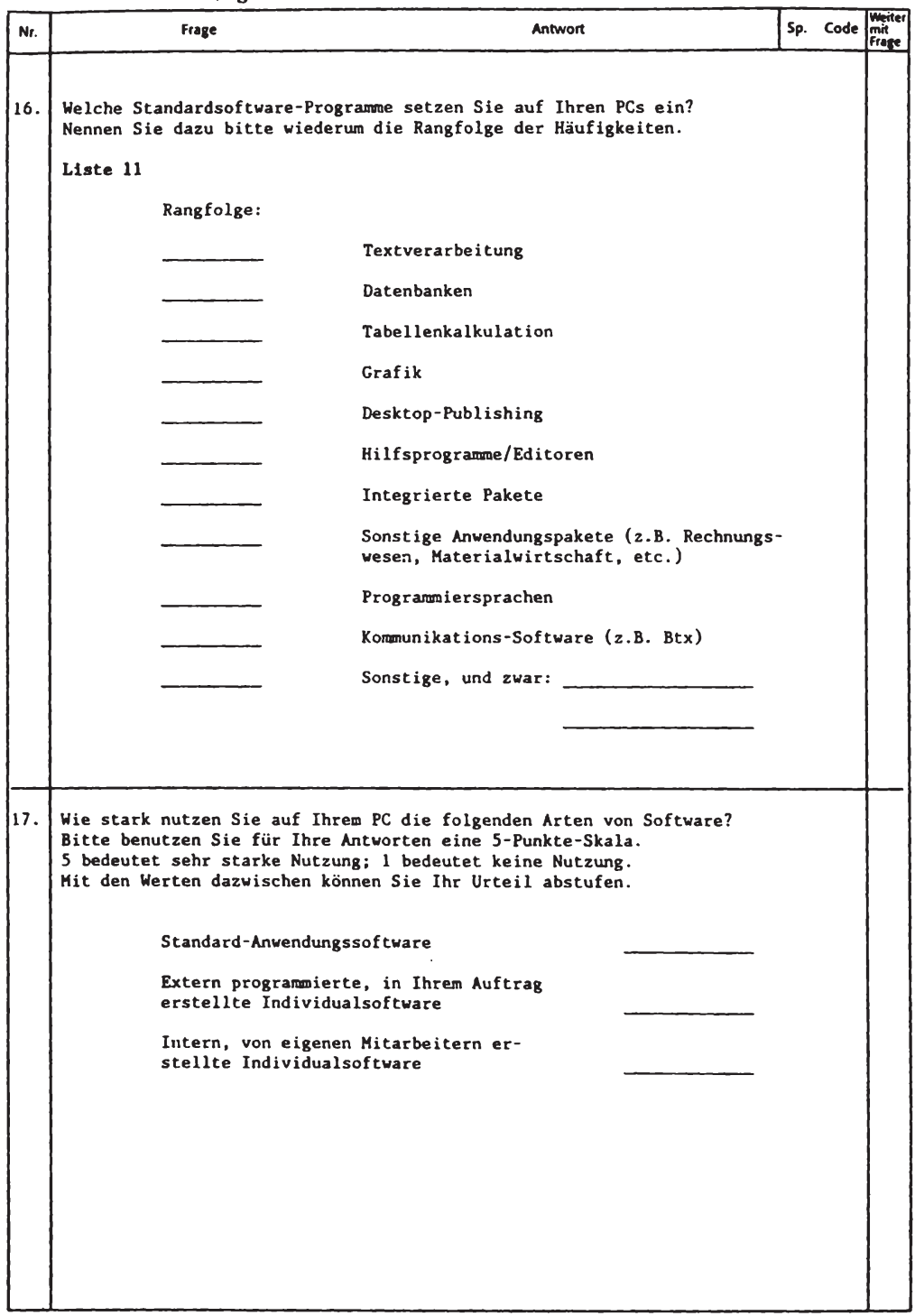


$91-2529 / v g-J u n i 1991$

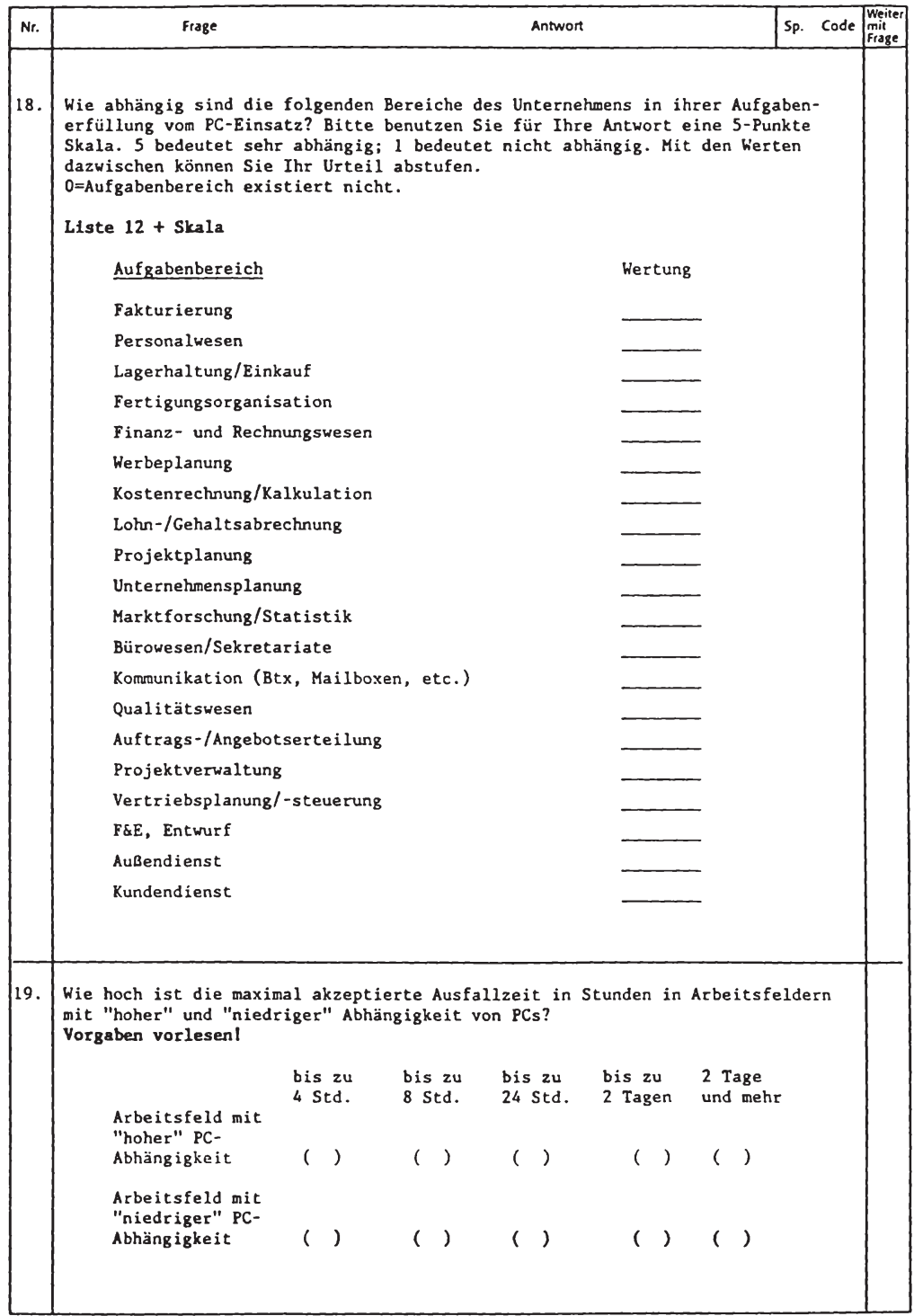


$91-2529 / v g$ - Juni 1991

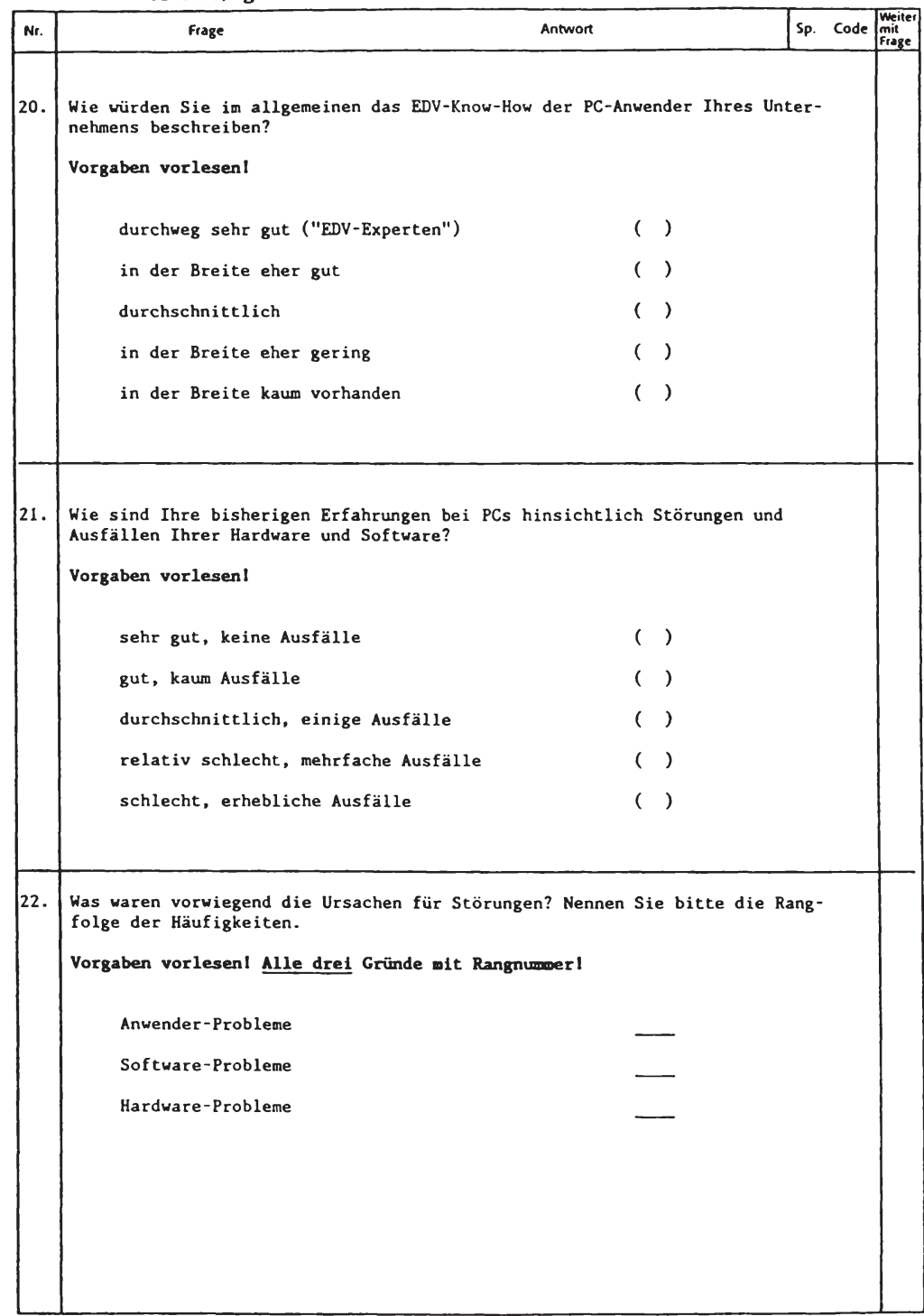


$91-2529 / v g$ - Juni 1991

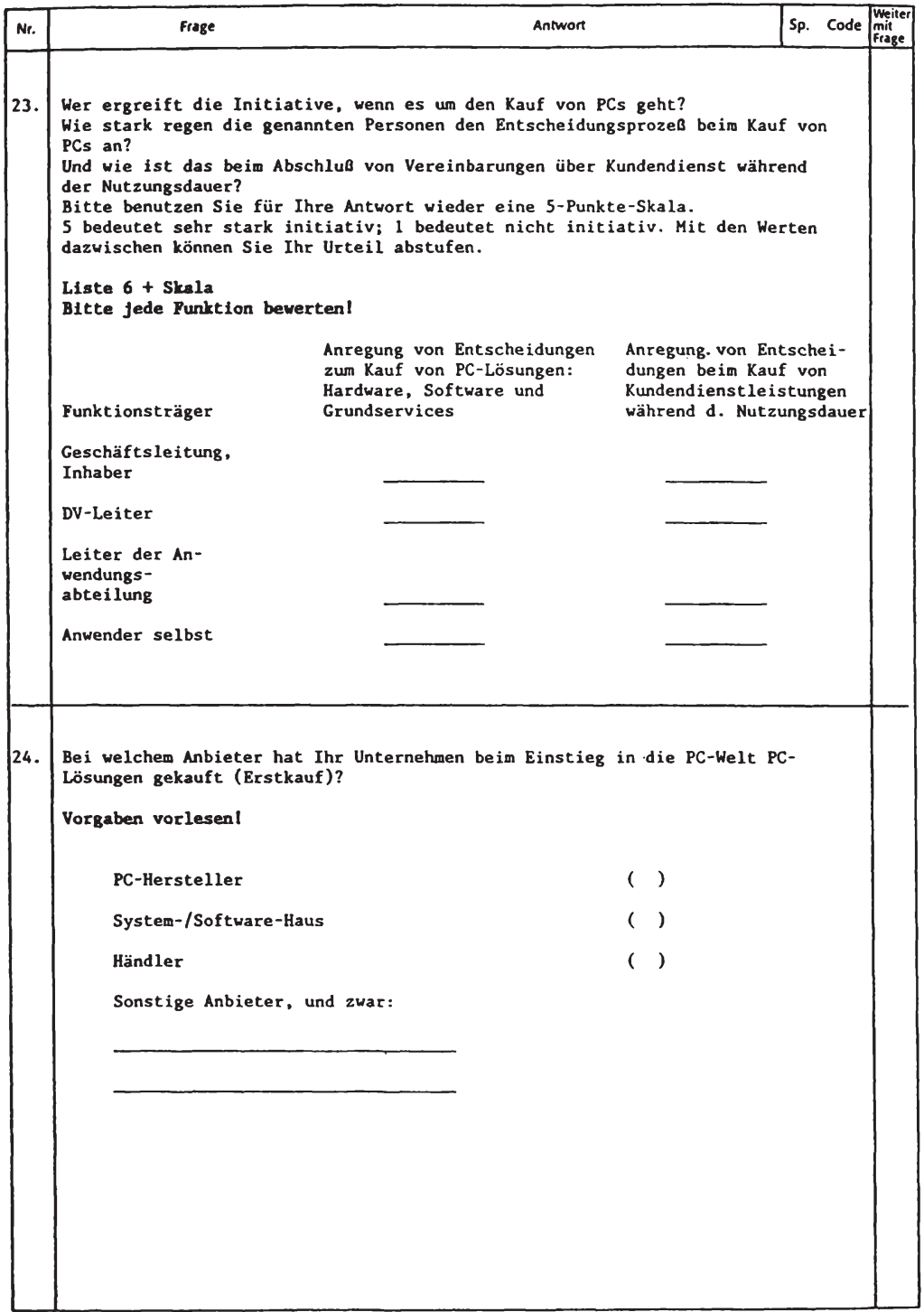


91-2529/vg - Juni 1991

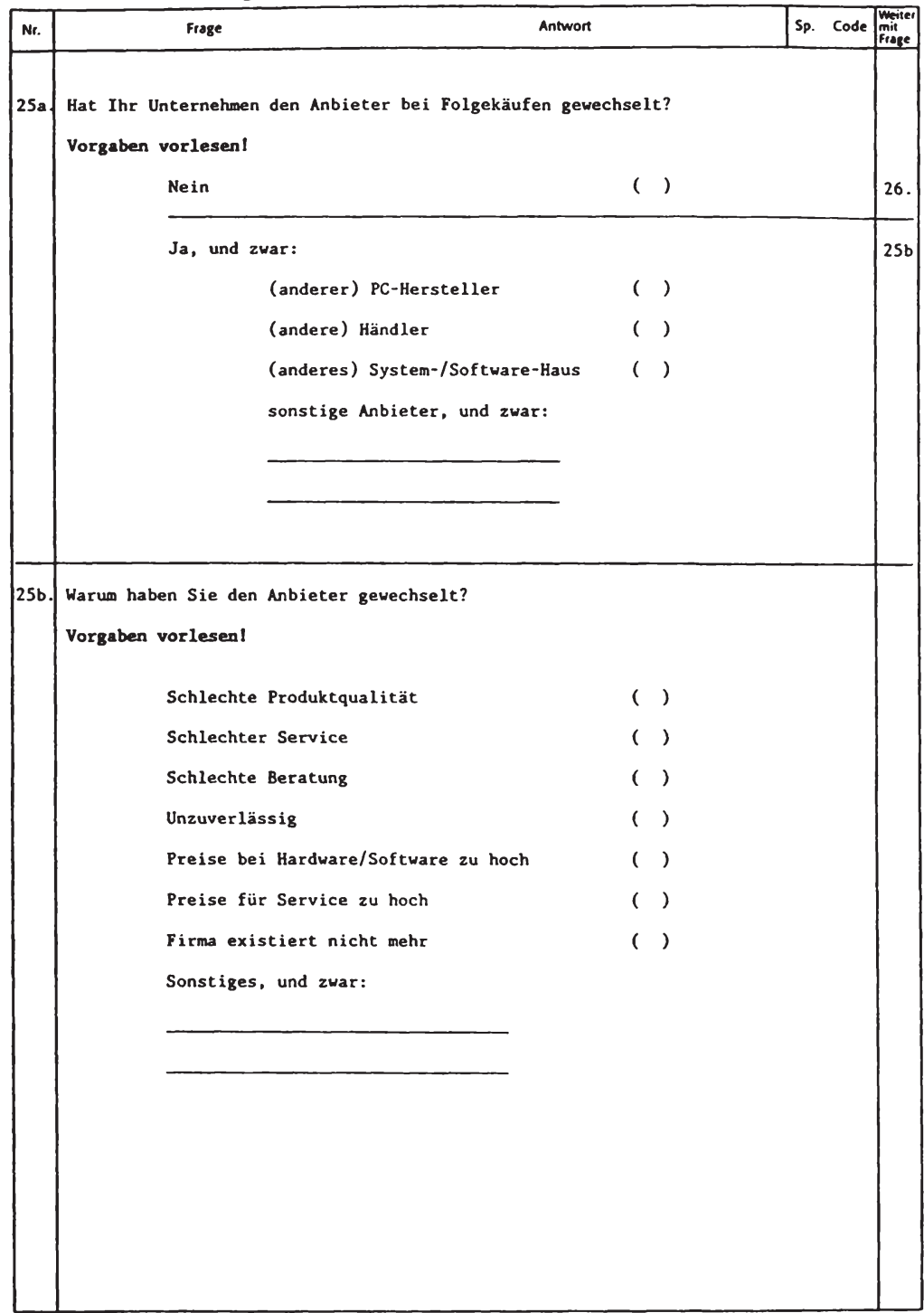


$91-2529 / v g-J u n i 1991$

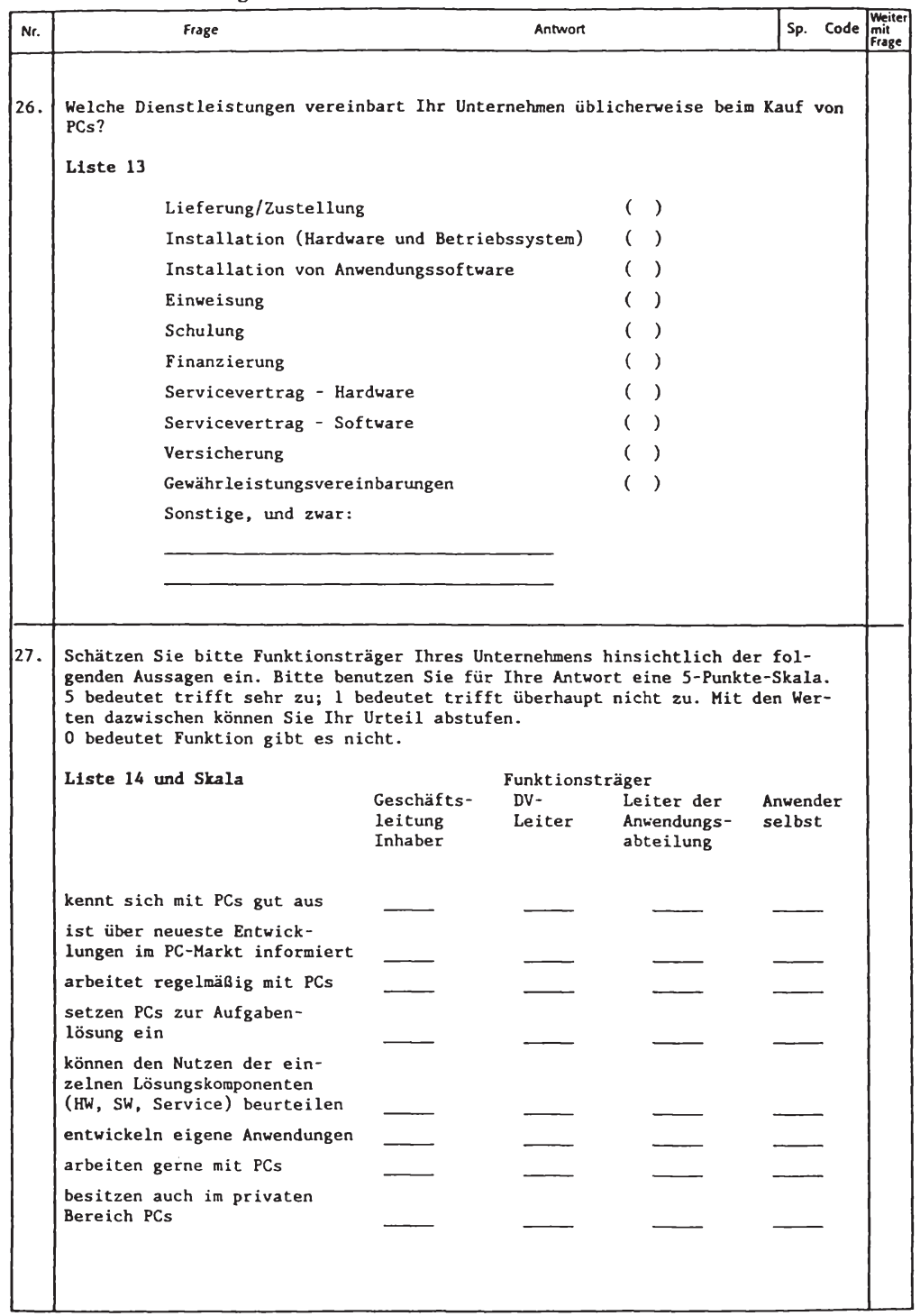


$91-2529 / v g-J u n i \quad 1991$

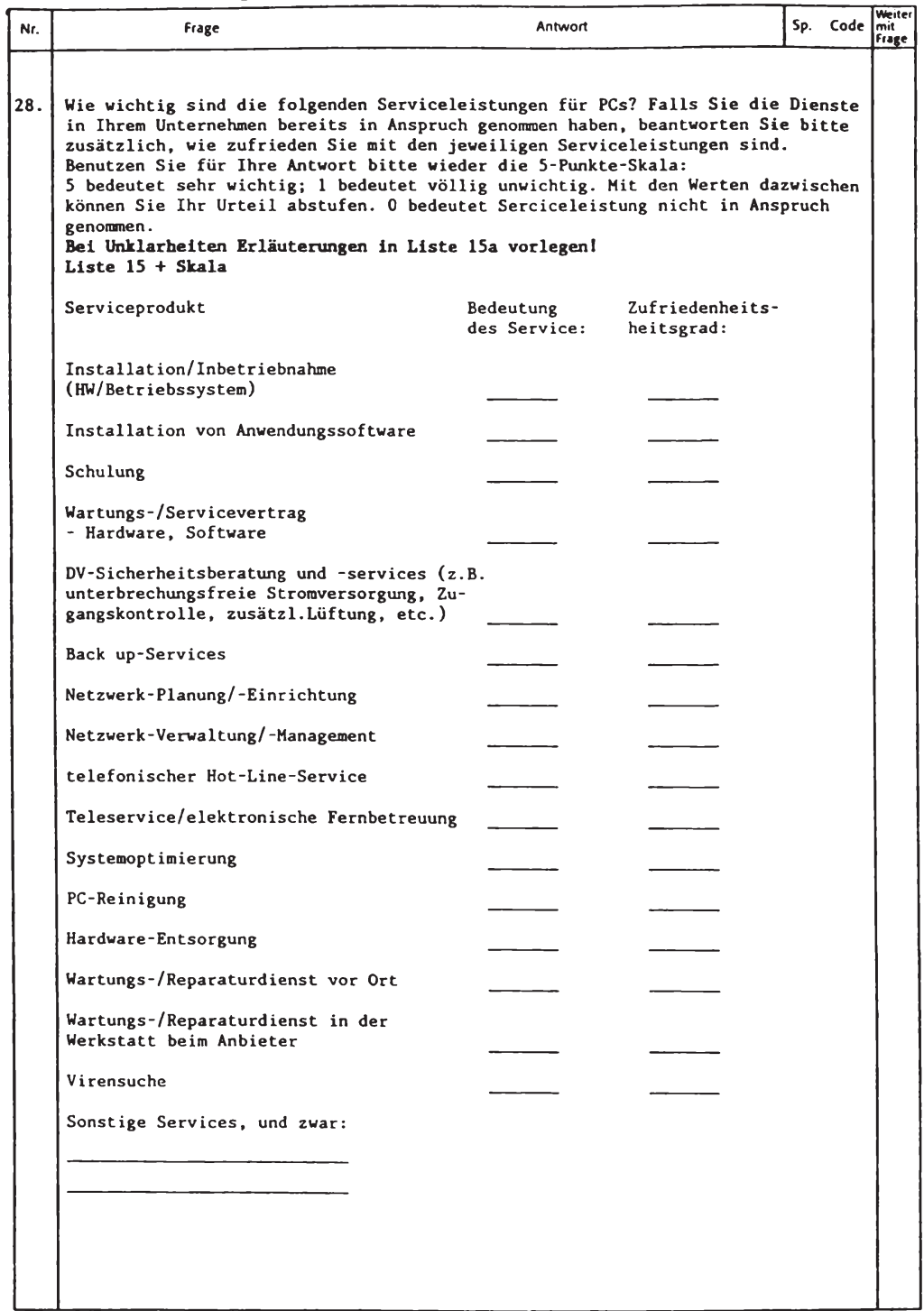


$91-2529 / v g-J u n i 1991$

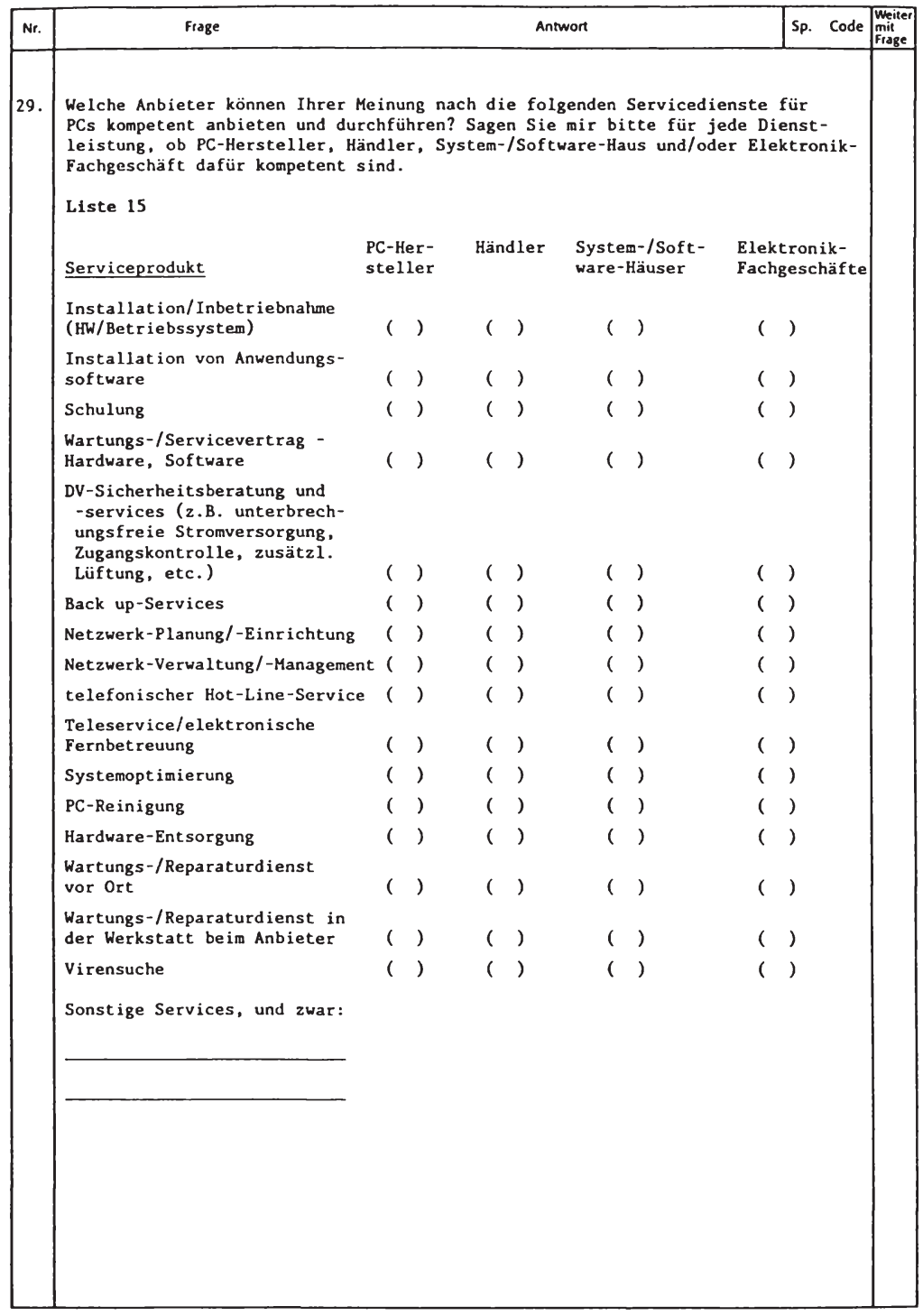




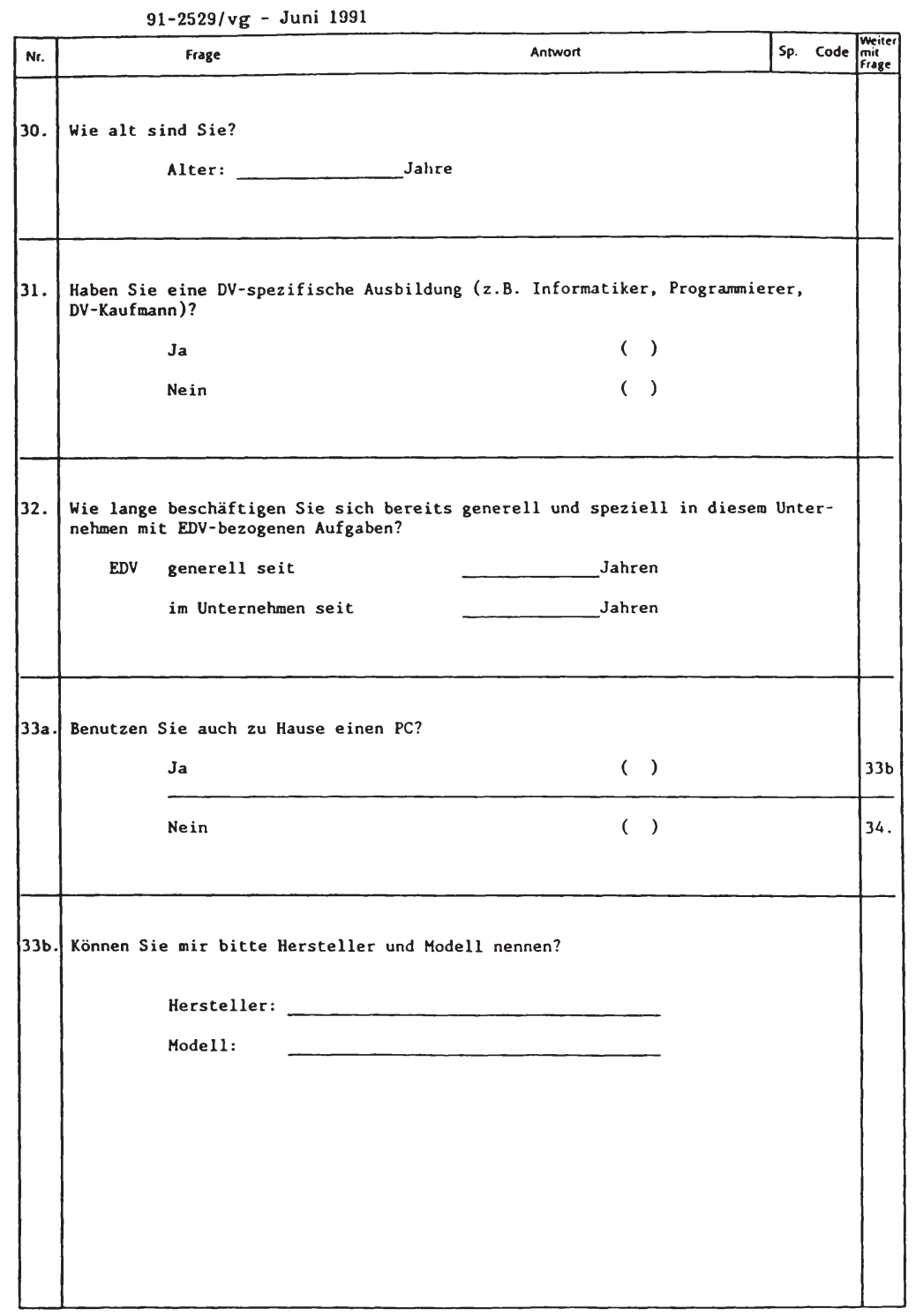


91-2529/vg - Juni 1991

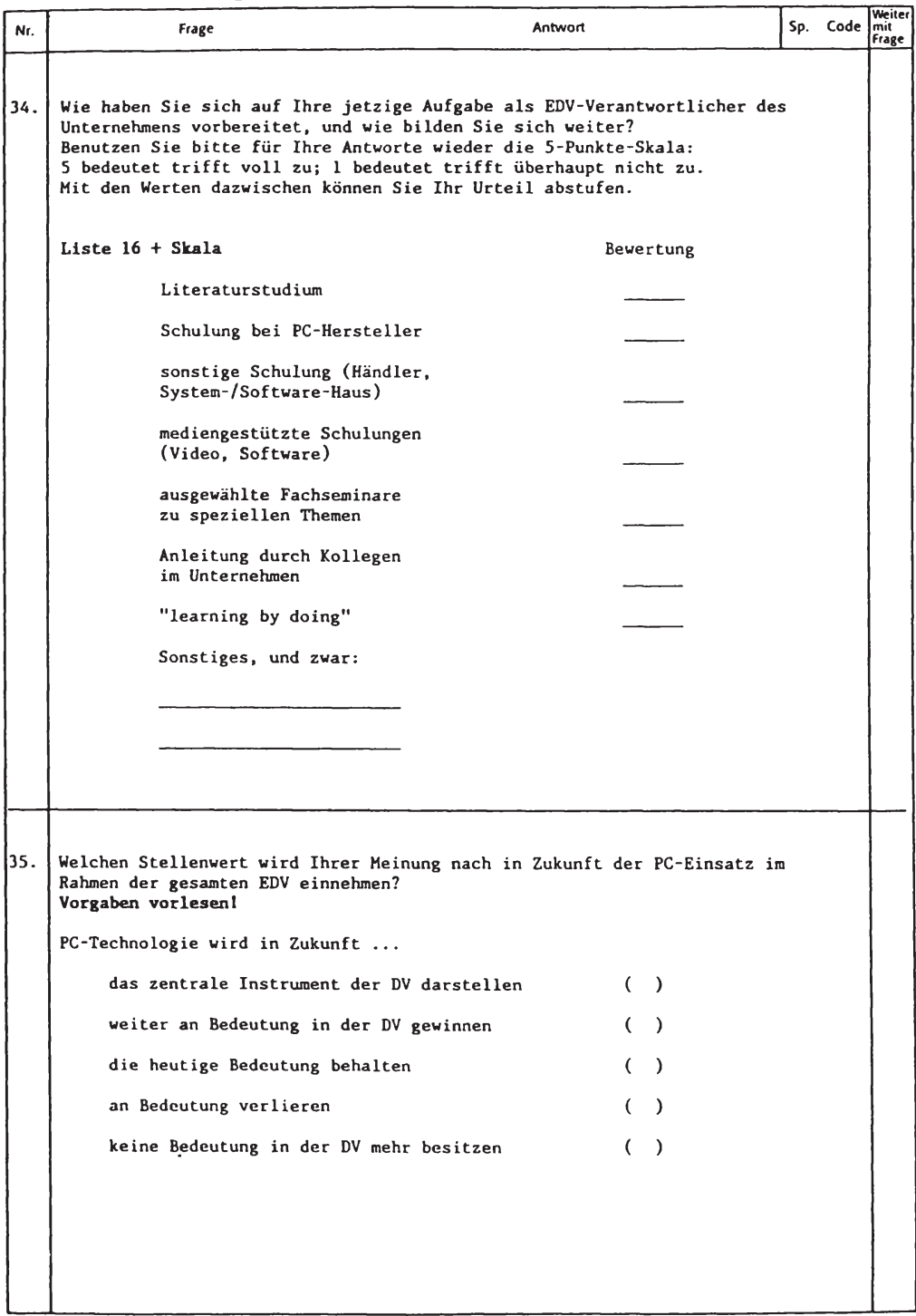


$91-2529 / v g-J u n i ~ 1991$

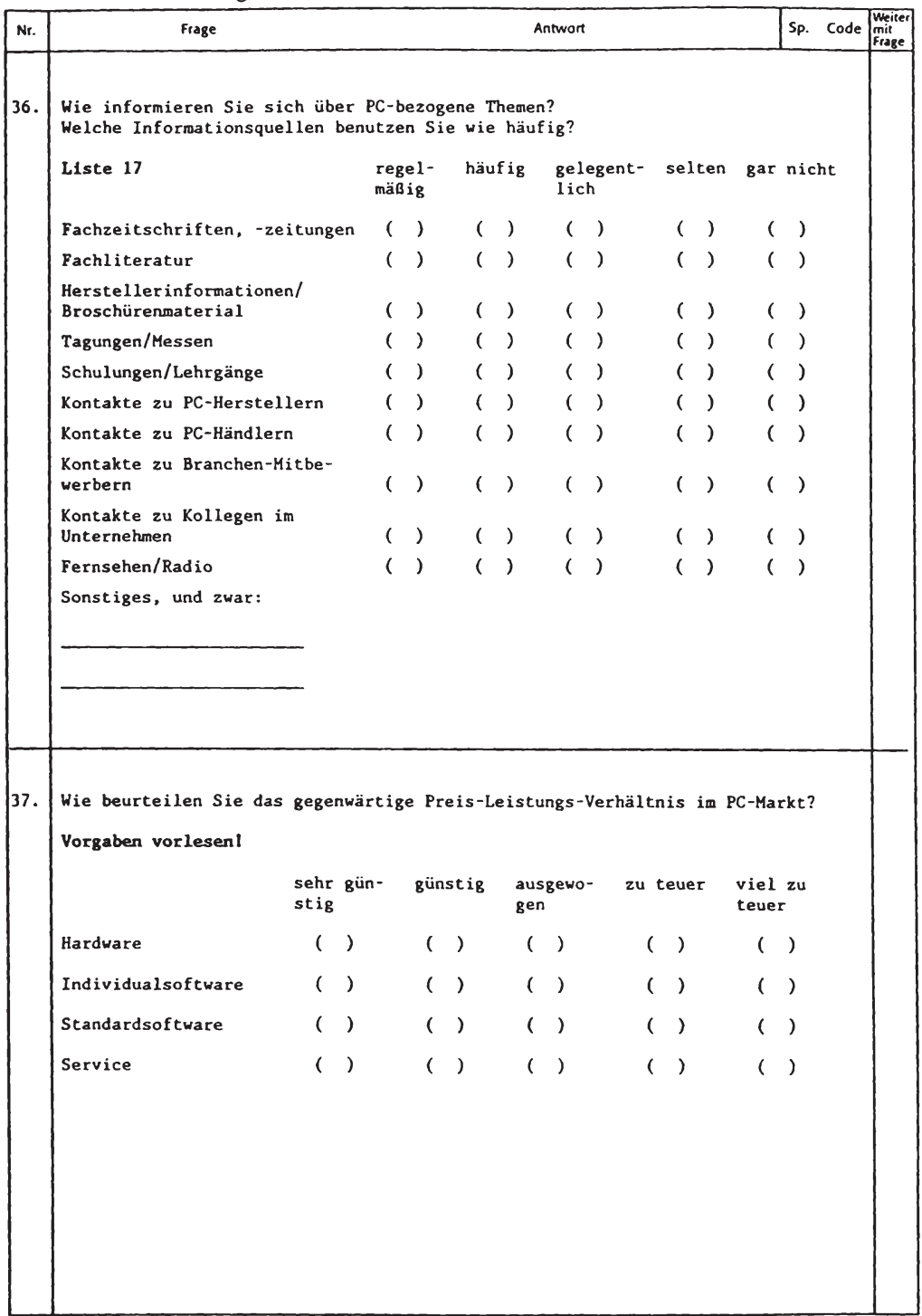


$91-2529 / v g-J u n i 1991$

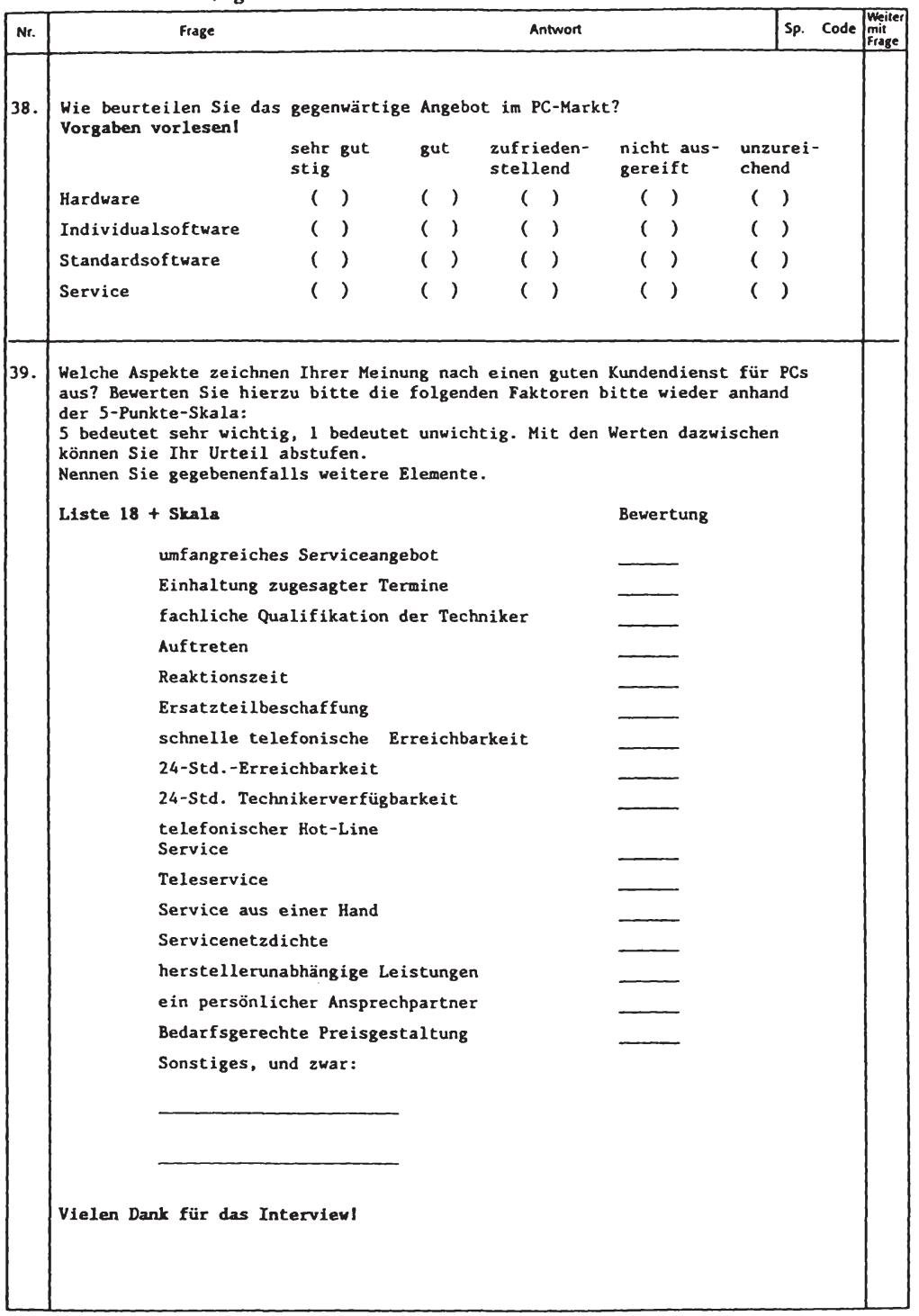




\section{LITERATURVERZEICHNIS}

Abratt, R.

Industrial Buying in High-Tech Markets, in: Industrial Marketing Management, 1986, Nr. 15, S. 293-298.

Ahlert, D.

Die Probleme der Abnehmerselektion und der differenzierten Absatzpolitik auf der Grundlage der segmentierenden Markterfassung, in: Der Markt, 1973, Nr. 4, S. 103-112.

Angehrn, 0 .

Marktsegmentierung als Absatzmethode, in: GFM, 14. Jg., 1968, Nr. 2, S. 38-42.

Atkinson, J.W.

Einführung in die Motivationsforschung, Stuttgart 1975.

Backhaus, $\mathrm{K}$.

Investitionsgütermarketing, 2. Auff., München 1990.

Backhaus, $\mathrm{K}$.

Multivariate Analysemethoden, 5. Aufi., Berlin, et al. Heidelberg 1990.

Backhaus, $\mathrm{K}$. Marktsegmentierungsprobleme in sich verānWeiber, R. dernden Märkten, in: VDI-Berichte, 1986, Nr. 616, S. 139-155.

Backhaus, $\mathrm{K}$. Systemtechnologie - Herausforderung des inWeiber, R. vestitionsgütermarketinn, in: HARVARDmanager, 1987, Nr. 4, S. 70-80.

Backhaus, K. Entwicklung einer Marketingkonzeption mit Weiber, $\mathrm{R}$. SPSS/PC +, Berlin u.a. 1989. 
Backhaus, $\mathrm{K}$.

Weiss, P.A.

Barnett, N.L.

Bass, F.M.

Tigert, D.J.

Lonsdale, R.T.

Bauer, E.

Bauer, E.

Bauer, $F$.

Baum, J.G.

Bazzi, R.

Pelz, N.

Becker, J.

Belz, Ch.

Benkenstein, $M$.
Kompetenz: Neue Dimension beim Marketing von Systemtechnologien, in: HARVARDmanager, 1989, Nr. 3, S. 107-114.

Beyond market segmentation, in: HBR, Vol. 47, January-February 1969, S. 152-166.

Market Segmentation: Group versus Individual Behavior, in: JoMR, Vol. 5, 1968, No. 3, S. 264-270.

Marktsegmentierung als Marketing-Strategie, Berlin 1976.

Markt-Segmentierung, Stuttgart 1977.

Datenanalyse mit SPSS, Berlin u.a. 1984.

Marktsegmentierung und Marktlücken, in: ZV+ZV, 67. Jg., 1970, S. 1243-1247.

Investitionsgüterservice - Direkt am Puls des Marketing, in: THEXIS, 1986, Nr. 1, S. 15-18.

Marketing-Konzeption: Grundlagen des strategischen Marketing-Managements, 2. Aufl., München 1988.

Kundendienst als Marketing-Instrument, in: THEXIS, 1986, Nr. 1, S. 2-6.

High Tech-Marketing, in: DBW, 50. Jg., 1990, S. 397-398. 
Bennewitz, H.I.

Bergs, S.

Berry, D.

Bleicher, $\mathrm{K}$.

Bleymüller, J.

et al.

Bleymüller, J.

Gehlert, G.

Gülicher, H.

Böhler, $\mathrm{H}$.

Bonoma, T.V.

Bonoma, T.V.

Shapiro, B.P.

Boyd, H.W.

Massy, W.F.

Braun, $\mathrm{K}$.

Lawrence, Ch.
Die Eigenständigkeit des absatzpolitischen Instrumentes Kundendienst und seine Bedeutung im modernen Marketingdenken, München 1968.

Optimalität bei Cluster-Analysen, Münster 1981.

Value added marketing emerging as key to competitiveness, in: Marketing News, 1987, Nr. 7, S. 3-14.

Kompetenz, in: Hrsg.: Grochla, E., Handwörterbuch der Organisation, 2. Aufl., Stuttgart 1980, Sp. 1056-1078.

Multivariate Analyse für Wirtschaftswissenschaftler, Münster 1983.

Statistik für Wirtschaftswissenschaften, 5. Aufl., München 1988.

Methoden und Modelle der Marktsegmentierung, Stuttgart 1977.

Major Sales: Who Really Does the Buying?, in: Harvard Business Review, May/June 1982, S. 111-119.

Segmenting the Industrial Market, Lexington, Mass./Toronto 1983.

Marketing Management, New York 1972.

TQM I: Ohne gemeinsame Werte wird es nichts, in: HARVARDmanager, 1993, Nr. 2, S. 77-85. 
Brockhoff, $\mathrm{K}$.

Buchner, D.

Bühner, R.

Büker, B.

Cordroch, C.

Corsten, $\mathbf{H}$.

Crone, B.

Davidow, W.H.

Davidow, W.H.

Uttal, B.

Dichtl, E.

Dichtl, E.

Müller-Heimann, G.
Technologischer Wandel und Unternehmenspolitik, in: ZfbF, 1984, S. 619-636.

Marketing und Diffusionsforschung, in: Der Marktforscher, 14. Jg., 1970, S. 12-16.

Betriebswirtschaftliche Organisationslehre, 3. Auff., München 1987.

Qualitätsbeurteilung investiver Dienstleistungen, Frankfurt/M., Bern, New York, Paris 1991.

Kundendienst unter Druck, in: Online, Nr. 1, 1988, S. 25-28.

Betriebswirtschaftslehre für Dienstleistungsunternehmungen, München u.a. 1988.

Marktsegmentierung, Bern 1977.

High Tech Marketing, Frankfurt/M. 1987.

So wird ihr Kundendienst unschlagbar, in: HARVARDmanager, 1990, Nr. 2, S. 14-21.

Ansatzpunkte zur Messung der Bedürfnisbefriedigung, in: Die Unternehmung, 29. Jg., 1975, Nr. 1, S. 1-21.

Konsumententypologische und produktorientierte Marktsegmentierung, in: Jahrbuch der Absatz- und Verbrauchsforschung, 1972, Nr. 4, S. 249-265. 
Dörner, D.

Egli, M.

Stutz, H.-R.

Eimer, E.

Engelhardt, W.H. Günter, B.

Finkelstein, W.

Guertin, R.J.A.

Forschner, G.

Frank, R.E.

Massy, W.F.

Wind, $Y$.

Freter, $\mathrm{H}$.

Frisch, W.

Fußbahn, K.-H.
Intention Memory and Intention Regulation, in: Klinx, F., Hagedorn, H., (Hrsg.), Human Memory and Cognitive Capability, North-Holland, Elsevier 1986.

Es lebe die Kundennutzenforschung, in: io-management Zeitschrift, 58.Jg., 1989, Nr. 1, S. 48-51.

Varianzanalyse, Stuttgart u.a. 1979.

Investitionsgütermarketing. Anlagen, Einzelaggregate, Teile, Roh- und Einsatzstoffe, Energieträger, Stuttgart, Berlin, Köln, Mainz 1981.

Integrated Logistics Support-The Design Engineering Link, Berlin u.a. 1988.

Investitionsgüter-Marketing mit funktionellen Dienstleistungen, Freiburg 1988.

Market Segmentation, Englewood Cliffs, N.J. 1972.

Marktsegmentierung, Stuttgart, Berlin, Köln, Mainz 1983.

Service-Management, Wiesbaden 1989.

Organisation und Steuerung des Technischen Kundendienstes - Profit- oder Costcenter?, in: Kundendienst-Management, Hrsg.: Meffert, $H_{\text {., }}$ Frankfurt/M., Bern 1982. 
Gemünden, H.G.

Wahrgenommenes Risiko und Informationsnachfrage, in: Marketing ZFP, 1985, Nr. 1, S. 27 38.

Gerstung, F.

Die Servicepolitik von Konsumgüterherstellern, Zürich 1974.

Gerstung, F.

Die Servicepolitik im Großhandel, Göttingen 1975.

Gerstung, F.

Die Servicepolitik als Instrument des Handelsmarketing, Göttingen 1978.

Gerth, E.

Betriebswirtschaftliche Absatz- und Marktforschung, Wiesbaden 1970.

GfK (Hrsg.)

Marktsegmentierung - ein Instrument zur Zielgruppenbestimmung, in: GfK-Sonderdienst, 1971, 2. Doppelheft, S. 39-80.

Ghemawat, P.

Dauerhafte Wettbewerbsvorteile aufbauen, in: HARVARDmanager, 1987, Nr. 2, S. 104-108.

Graumann, J.

Die Managementaufgabe der Zukunft: DienstGebhardt,P. leistungsmarketing, in: Markenartikel, 1987, Nr. 10, S. $486-492$.

Green, P.E.

Benefit Bundle Analysis, in: JoAR, Wind, $Y$. Jain, A. Vol. 12, 1972, Nr. 2, S. 31-36.

Gröne, A.

Marktsegmentierung bei Investitionsgütern, Wiesbaden 1977.

Guilford, J.P.

Persönlichkeit, Weinheim 1954. 
Håkansson, $\mathrm{H}$. Johanson, J. Wootz, B.

Haley, R.I.

Haller, $M$.

Hammann, P.

Hammann, P.

Hammann, P.

Hammann, P. Erichson, B.

Hampl, R.K.

Hansen, $U$.
Influence Tactics in Buyer-Seller

Processes, in: Industrial Marketing

Management, Vol. 5, 1976, Nr. 6, S. 319-332.

Benefit Segmentation: A Decisionoriented Research Tool, in: JoM, 32. Jg., 1968, Nr. 7, S. $30-$ 35.

Kundenorientierung zwischen High Tech und High Touch, in: THEXIS, 1986, Nr. 1, S. 10-14.

Marktforschung für Investitionsgüter, in: Anlagen-Marketing, Sonderheft Nr. 7 der ZfbF, Hrsg.: Engelhardt, W.H., Lassmann, G., Opladen 1977.

Das Optimierungsproblem im Kundendienst Aussagewert und Stand der Diskussion, in: Kundendienst-Management, Hrsg.: Meffert, H., Frankfurt/M., Bern 1982.

Marktsegmentierung, in: Technischer Vertrieb, Hrsg.: Plinke, W., Projektgruppe Technischer Vertrieb, Freie Universitāt Berlin, Berlin 1985.

Marktforschung, Stuttgart, New York 1978.

Zunehmende Bedeutung des technischen Kundendienstes (TKD), in: Maschinenbau-Nachrichten (NDMA), 1988, Nr. 9, S. 7-19.

Absatz- und Beschaffungsmarketing des Einzelhandels. Eine Aktionsanalyse, Teil 1 und 2, Göttingen 1976. 
Hart, Ch.W.

Heskett, J.L.

Dasser, W.E.

Heinemann, G.

Hilke, W.

Hink, A.

Stiller, $H$.

Hofer, Ch.W.

Schendel, D.

Horst, B.

Hummel, S.

Mānnel, W.

Input (Hrsg.)

Jacob, R.

Jacob, R.
Wie Sie aus Pannen Profit ziehen, in: HARVARDmanager, 1991, Nr. 1, S. 128-136.

Betriebstypenprofilierung und Erlebnishandel, Wiesbaden 1989.

Dienstleistungs-Marketing, Schriften zur Unternehmensführung, Bd. 35, Hrsg.: Jacob, H. U.a., Wiesbaden 1989.

Consumer Electronic Show Chicago Abenteuer Chicago, in: ASM, 7. Jg., 1992, Nr. 8, S. $40-45$.

Strategy Formulation, St. Paul, Minnesota 1978.

Ein mehrdimensionaler Ansatz zur Segmentierung von Investitionsgütermärkten, Köln 1988.

Kostenrechung 1 - Grundlagen, Aufbau und Anwendung, 4. Aufl., Wiesbaden 1986.

Value Added Customer Service Products, 0.O. 1985.

Marktsegmentierung auf Investitionsgütermärkten als Grundlage der Steuerung absatzpolitischen Mitteleinsatzes. Dargestellt am Beispiel des Lastkraftwagenmarktes, Bern, Frankfurt/M. 1972.

Gezielte Investgüter-Politik für Marktsegmente, in: Marketing Journal, 1973, Nr. 6, S. 476-479. 
Jacoby, A.

Kähler, W.-M.

Kaiser, $\mathbf{A}$.

Kern, E.

Kirchgeorg, $M$.

Koch, W.

Kols, P.

Konrad, E.

Kotler, P.

Kotler, $\mathrm{P}$.

Kratz, J.
Kundendienst - Der erfolgreiche Verkäufer im Detailhandel, Schweizer Schriften für rationelles Wirtschaften, Nr. 4, Zürich 1927.

Statistische Datenanalyse mit SPSS/PC ${ }^{+}$, Braunschweig 1990.

Die Identifikation von Marktsegmenten, Berlin 1978.

Der Interaktionsansatz im Investitionsgütermarketing, Berlin 1990.

Ökologieorientiertes Unternehmensverhalten Typologien und Erklärungsansätze auf empirischer Grundlage, Wiesbaden 1989.

Grundlagen und Technik des Vertriebs, Bd. 1, Berlin 1958.

Bedarfsorientierte Martsegmentierung auf Produktivgütermärkten, Frankfurt/M. 1986.

Kundendienstpolitik als Marketinginstrument von Konsumgüterherstellern, Zürich 1974.

Marketing Management: Analysis, Planning and Control, 4th ed., Pretice Hall, Englewood Cliffs, N.J. 1978.

Marketing Management, 4. Aufl., Englewood Cliffs, N.J. 1980.

Der Interaktionsprozess beim Kauf von einzeln gefertigten Investitionsgütern, Bochum 1975. 
Kroeber-Riel, W.

Lawer, E.E.

Levitt, Th.

Lienert, G.A.

Lignel, J.

Cadet, $\mathbf{A}$.

Lingenfelder, $\mathbf{M}$.

Schneider, W.

Little, A.D.

Little, A.D.

Lo, L.

Lovelock, Ch.

Lux, P.G.C.
Konsumentenverhalten, 4. Aufl., München 1990.

Motivation in Work Organisations, Montrey, Cal. 1973.

Der Verkaufsabschluß ist erst ein Anfang, in: HARVARDmanager, 1985, Nr. 1, S. 15-21.

Testaufbau und Testanalyse, 3. Aufl., Weinheim 1969.

The Problems of Market Segmentation, in: The European Marketing Research Review, Vol. 2, 1967, No. 2, S. 24-30.

Die Kundenzufriedenheit, in: Marketing ZFP, 1991, Nr. 2, S. 109-119.

Innovation als Führungsaufgabe, Frankfurt/M., New York 1988.

Management der F\&E-Strategie, Wiesbaden 1991.

Prognoseinformationen für kundendienstpolitische Entscheidungen - dargestellt am Beispiel des Fotomarktes, Münster 1979.

Dienstleister können Effizienz und Kundenzufriedenheit verbinden, in: HARVARDmanager, 1993, Nr. 2, S. 68-75.

Zur Durchführung von Corporate Identity Programmen, in: Birkigt, K., Stadler, M.M., Funcke, H.J., (Hrsg.), Corporate Identity: Grundlagen, Funktionen, Fallbeispiele, 4. Aufl., Landsberg am Lech 1988. 
Lynn, S.A.

Martin, C.R.

Wright, R.L.

Massy, W.F.

Meder, N.,

Scheuber, P.

Meffert, $\mathrm{H}$.

Meffert, $H$.

Meffert, $H$.

Meffert, $\mathrm{H}$.

Meffert, $\mathrm{H}$.

Meffert, $H$.
Segmenting a Business Market for a Professional Service, in: Industrial Marketing Management, Vol. 15, 1986, S. 13-21.

Profit-Oriented Data Analysis for Market Segmentation, in: JoMR, Vol. 11, 1974, No. 8, S. 237-242.

Marketing Management, New York 1972.

MS-OS/2-Presentation-Manager: Einführung und Überblick, Haar bei München 1990.

Der Kundendienst als Marketinginstrument Einführung in die Problemkreise des Kundendienst-Managements, in: Kundendienst-Management, Hrsg.: Meffert, H., Frankfurt/M., Bern 1982.

Interdependenzen und Koordination zwischen Marketing und Kundendienst, in: KundendienstManagement, Hrsg.: Meffert, H., Frankfurt/M., Bern 1982.

Die Bedeutung von Konkurrenzstrategien im Marketing, in: Marketing ZfP, Heft 1, 1985, S. 13-21.

Marketing, 7. Aufl., Wiesbaden 1986.

Kundendienstpolitik, in: Marketing ZFP, 9. Jg., 1987, Nr. 2, S. 93-102.

Medien 2000 - Thesen zu den Entwicklungsperspektiven elektronischer Medien im Marketing, in: Strategische Unternehmensführung und Marketing, Hrsg.: Meffert, H., Wiesbaden 1988. 
Meffert, $\mathrm{H}$.

Meffert, $\mathrm{H}$.

Meffert, $\mathrm{H}$.

Mertens, P.

Schumann, $M$.

Hohe, U.

Meyer, $\mathbf{A}$.

Meyer, A.

Millar, V.E.

Mühlbacher, $\mathrm{H}$.

Botschen, $\mathrm{G}$.

Müller-Hagedorn, L.

Myers, J.H.
Strategische Unternehmensführung und Marketing, Wiesbaden 1988.

Marketingforschung und Käuferverhalten, 2. Aufl., Wiesbaden 1992.

Marktorientierte Führung von Dienstleistungsunternehmen - neue Entwicklungen in Theorie und Praxis, Arbeitspapier Nr. 78 der wissenschaftlichen Gesellschaft für Marketing und Unternehmensführung e.V., Hrsg.: Meffert, H., Wagner, H., Backhaus, K., Münster 1993.

Informationstechnik als Mittel zur Verbesserung der Wettbewerbsposition - Erkenntnisse aus einer Beispielsammlung, in: Informationstechnologie und strategische Führung, Hrsg.: Spremann, K., Zur, E., Wiesbaden 1989.

Produktdifferenzierung durch Dienstleistungen, in: Marketing ZFP, 1985, S. 99-107.

Dienstleistungsmarketing, 3. Aufl., Augsburg 1988.

Wettbewerbsvorteile durch Information, in: HARVARDmanager, 1986, Nr. 1, S. 149-156.

Benefit-Segmentierung von Dienstleistungsmärkten, in: Marketing ZFP, 12. Jg., 1990, Nr. 3, S. $159-168$.

Das Konsumentenverhalten, Wiesbaden 1986.

Benefit Structure Analysis: A new Tool for Product Planning, in: JoM, Vol. 40, 1969, No. 4, S. 23-32. 
Nagel, K.

Nixdorf

Computer AG (Hrsg.)

O.V.

O.V.

O.V.

O.V.

O.V.

O.V.

O.V.

O.V.

O.V.
Bewertung stategischer Wettbewerbsvorteile durch Informationssysteme, in: Informationstechnologie und strategische Führung, Hrsg.: Spremann K., Zur, W., Wiesbaden 1989.

Kundendienst - Kaufmännischer Produktbericht 6/89, Paderborn 1989.

Marktforscher warnen die Industrie: PC wird härter, in: Chip, 1990, Nr. 11, S. 42.

Service bringt das Geld, in: Chip, 1991, Nr. 4, S. 13.

PC-Markt in Deutschland, Vobis ist spitze, in: Chip, 1991, Nr. 5, S. 14.

Das Geschäft der 90er Jahre, in: Chip, 1991, Nr. 7, S. 11.

Bus-Produkte stark gefragt, in: Chip, 1991, Nr. 10, S. 13.

Windows stärkt die Nachfrage, in: Chip, 1992, Nr. 1, S. 14.

Barometer Prognose für Betriebssysteme - DOS bleibt Spitze, in: Chip, 1992, Nr. 2, S. 14.

EDV-Markt in Deutschland - Lage nicht schlecht, in: Chip, 1992, Nr. 4, S. 10-11.

Wachstum durch Netze, in: Chip, 1992, Nr. 9, S. 14. 
O.V.

Ostmeier, $\mathrm{H}$.

Plank, R.E.

Poggenpohl, M.

Porter, M.E.

Porter, M.E.

Porter, M.E.

Millar, V.E.

Quinn, J.B.

Gagnon, Ch.E.

Quinn J.B.

Baruch, J.J.

Paquette, P.C.

Raffio, T.
Rückgang bei Großrechnern - Verlierer IBM, in: Chip, 1993, Nr. 3, S. $26-27$.

Ökologieorientierte Produktinnovationen, Frankfurt/M., Bern, New York, Paris 1990.

A Critical Review of Industrial Market Segmentation, in: Industrial Marketing Management, 1985, No. 14, S. 79-91.

Verbundanalyse im Einzelhandel auf Grundlage von Kundenkarteninformationen. Eine empirische Untersuchung von Verbundbeziehungen zwischen Abteilungen, (unveröffentliches Manuskript), Münster 1993.

Wettbewerbsstrategie, Frankfurt/M. 1983.

Wettbewerbsvorteile, Frankfurt/M., New York 1986.

Wettbewerbsvorteile durch Information, in: HARVARDmanager, 1986, Nr. 1, S. 149-160.

Die Dienstleisungen werden automatisiert, in: HARVARDmanager, 1987, Nr. 2, S. 74-81.

Exploiting a Manufacturing-Service Interfaces, in: Sloan Management Review, 1988, No. 4, S. 45-56.

TQM II: Wie Delta Dental Plan Service Excellence erreichte, in: HARVARDmanager, 1993, Nr. 2, S. 86-97. 
Reichheld, F.F.

Sasser, W.E.

Remmerbach, K.U.

Reuter, E.

Rodgers, E.M.

Sahal, D.

Schlesinger, L.A.

Heskett, J.L.

Scheuch, F.

Scheuch, F.

Scheuch, F.

Schierenbeck, $H$.

Schönrock, A.
Zero-Migration: Dienstleister im Sog der Qualitätsrevolution, in: HARVARDmanager, 1991, Nr. 4, S. 108-116.

Value Added Marketing, in: Marktforschung \& Management, 1990, Nr. 2, S. 13-21.

Wertschöpfung durch Informationstechnologie, Hrsg.: Spremann, K., Zur, E., Wiesbaden 1989.

Diffusion of Innovations, 3. Aufl., New York 1983.

Patterns of Technological Innovation, London u.a. 1981.

The Service-Driven Service Company, in:

Harvard Business Review, 1991, No. 10/11, S. 71-81.

Logische Struktur und pragmatische Bedeutung der Marktsegmentierung, in: Die Unternehmung, 28. Jg., 1974, Nr. 3, S. 213-230.

Investitionsgŭter-Marketing, Opladen 1975.

Dienstleistungsmarketing, Mūnchen 1982.

Grundzüge der Betriebswirtschaftslehre, 9. Aufl., München 1987.

Die Gestaltung des Leistungsmix im marktorientierten Kundendienst, in: Kundendienst-Management, Hrsg.: Meffert, H., Frankfurt/M., Bern 1982. 
Schubert, F.

Schubö, W.

et. al.

Schüler, W.

Schulz, D.

Schwenker, B.

Sheth, J.

Smith, W.R.

Speckmann, R.E.

Spremann, K. Reichling, $P$.
Akzeptanz von Bildschirmtext in Unternehmen und im Markt, Münster 1986.

Handbuch der Programmversion 4.0 und SPSS-X 3.0, Stuttgart, New York 1991.

Informationsmanagement: Gegenstand und organisatorische Konsequenzen, in: Informationstechnologie und strategische Führung, Hrsg.: Spremann, K., Zur, E., Wiesbaden 1989.

Kaufentscheidungsprozesse des Konsumenten, Wiesbaden 1972.

Dienstleistungsunternehmen im Wettbewerb, Wiesbaden 1989.

Marktsegmentierung als relvante Planungshilfe des Marketing - Marktsegmentierung als Strategie des Marketing, in: Jahrbuch der Absatzund Verbraucherforschung, 1972, Nr. 2, S. 129144.

Product Differentation and Market Segmentation as Alternative Marketing Stategies, in: JoM, Vol. 21, 1956, No. 1, S. 1-8.

Segmenting Buyers in Different Types of Organisations, in: Industrial Marketing Management, Vol. 10, 1981 , No. 10, S. 43-48.

Schwachstellen und Konstruktionsprinzipien beim Systemdesign, in: Informationstechnologie und strategische Führung, Hrsg.: Spremann, K., Zur, E., Wiesbaden 1989. 
Stauss, $\mathbf{B}$.

Stăudel, Th.

Stäudel, Th.

Stäudel, Th.

Steinhausen, D.

Langer, $\mathrm{K}$.

Steinhausen, D.

Zörkendörfer, S.

Strothmann, K.-H.

Strothmann, K.-H.

Kliche, $M$.

Theisen, $\mathbf{P}$.

Thiesing, E.-O.
Kundendienstqualität als Erfolgsfaktor im Wettbewerb, in: THEXIS, 1991, Nr. 2, S. 47-51.

Problemlösen, Emotion und Kompetenz, Regensburg 1987.

Der Kompetenzfragebogen, in: Diagnostica, 1988, Nr. 34, S. 136-148.

Emotion, Kompetenz - Vertrauen in sich selbst, in: Absatzwirtschaft, Sondernummer Oktober 1988, S. 148-154.

Clusteranalyse, Berlin, New York 1977.

Statistische Datenanalyse mit dem Programmsystem SPSS-X und SPSS/PC ${ }^{+}, 2$. Aufl., München, Wien 1990.

Investitionsgütermarketing, München 1979.

Marktsegmentierung für High-Tech-Anbieter, in: Marktforschung \& Management, 1989, Nr. 3 , S. 82-87.

Kundendienstpolitik, in: HWA, Hrsg.: Tietz, B., Stuttgart 1974, Sp. 1155-1165.

Strategische Marketingplanung in filialisierten Universalbanken. Integrierte Filial- und Kundengruppenstrategien auf der Grundlage erfolgsbeeinflussender Schlüsselfaktoren, Frankfurt/M., Bern, New York, Paris 1986. 
Töpfer, A.

Vogel, F.

Weber, M.R.

Wegwart, J.

Weiss, P.A.

Wilkie, W.L.

Wilson, D.T.

Wind, $Y$.

Wind, Y.

Cardozo, R.
Industrial Market Segmentation, in: Industrial

Marketing für Start-up-Geschäfte mit Technologieprodukten, in: Technologie Marketing, Hrsg.: Töpfer, A., Sommerlatte, T., Landsberg am Lech 1991.

Probleme und Verfahren der numerischen Klassifikation, Göttingen 1975.

Erfolgreiches Service-Management: Gewinnbringende Vermarktung von Dienstleistungen,

Preis- und Kontrahierungspolitik im Kundendienst unter besonderer Berücksichtigung von Wartungs- und Call-Service, in: KundendienstManagement, Hrsg.: Meffert, H., Frankfurt/M., Bern 1982.

Die Kompetenz von Systemanbietern, Berlin 1992.

The "Product Stream" of Market Segmentation. A Research Approach, in: Relevance in Marketing. Marketing in Motion. Combined Proceedings of the Spring and Fall Conferences 1971, Hrsg.: Allvine, F.C., Chicago 1972, S. 317-321.

Industrial Buyers Decision-Making Styles, in: JoMR, Vol. 8, 1971, Nr. 4, S. 433-436.

Issues and Advances in Segmentation Research, in: JoMR, Vol. 15, 1978, No. 8, S. 317-337.

Marketing Management, Vol. 3, Oktober 1974, Nr. 1, S. 153-166. Landsberg am Lech 1989. 
Winkler, A.

Winter, F.W.

Witte, E.

Witte, E.

Wöhe, G.

Yankelovich, D.

Young, $A$.

Evens, $M$.

Orr, J.

Zayac, B.P.

Summers, R.C.

Zerr, K.

Jugel, S.

Zerr, K.

Jugel, $\mathrm{S}$.
Praktische Anwendungen der Marktsegmentierung. Marktsegmentierung als Methode der Marktforschung, in: Jahrbuch der Absatz- und Verbraucherforschung, 1972, Nr. 2, S. 101-120.

A Cost-Benefit Approach to Market Segmentation, in: JoM, Vol. 43, 1979, No. 4, S. 103-111.

Organisation für Innovationsentscheidungen das Promotorenmodell, Göttingen 1973.

Kraft und Gegenkraft im Entscheidungsprozeß, in: ZfB, 1976, Nr. 46, S. 319-326.

Einführung in die allgemeine Betriebswirtschaftslehre, 14. Aufl., München 1981.

Neue Kriterien der Marktsegmentierung, in: Marketing-Management und Organisation, Hrsg.: Britt, S.H., Boyd, H.W., München 1971, S. 209-225.

Management Awareness Of Computer Risks, o.O. 1987.

Computers and Security, o.O. 1988.

Dienstleistung als strategisches Element eines Technologie Marketing. Arbeitspapier Nr. 68 des Instituts für Marketing der Universität Mannheim, Mannheim 1989.

Dienstleistungen als strategisches Element eines Technologie Marketing, in: Marketing ZFP, 1989, Nr. 3, S. 162-164. 
Zimmermann, G.

Grundzüge der Kostenrechung, 2. Aufl., Stuttgart 1982.

Zörgiebel, W.W.

Technologie in der Wettbewerbsstrategie, Berlin 1983. 


\section{SCHRIFTEN ZUM MARKETING}

Band 1 Friedrich Wehrle: Strategische Marketingplanung in Warenhäusern. Anwendung der Portfolio-Methode. 1981. 2. Auflage. 1984.

Band 2 Jürgen Althans: Die Übertragbarkeit von Werbekonzeptionen auf internationale Märkte. Analyse und Exploration auf der Grundlage einer Befragung bei europaweit tătigen Werbeagenturen. 1982 .

Band 3 Günter Kimmeskamp: Die Rollenbeurteilung von Handelsvertretungen. Eine empirische Untersuchung zur Einschătzung des Dienstleistungsangebotes durch Industrie und Handel. 1982.

Band 4 Manfred Bruhn: Konsumentenzufriedenheit und Beschwerden. Erklärungsansătze und Ergebnisse einer empirischen Untersuchung in ausgewählten Konsumbereichen. 1982.

Band 5 Heribert Meffert (Hrsg.): Kundendienst-Management. Entwicklungsstand und Entscheidungsprobleme der Kundendienstpolitik. 1982.

Band 6 Ralf Becker: Die Beurteilung von Handelsvertretem und Reisenden durch Hersteller und Kunden. Eine empirische Untersuchung zum Vergleich der Funktionen und Leistungen. 1982.

Band 7 Gerd Schnetkamp: Einstellungen und Involvement als Bestimmungsfaktoren des sozialen Verhaltens. Eine empirische Analyse am Beispiel der Organspendebereitschaft in der Bundesrepublik Deutschland. 1982.

Band 8 Stephan Bentz: Kennzahlensysteme zur Erfolgskontrolle des Verkaufs und der MarketingLogistik. Entwicklung und Anwendung in der Konsumgüterindustrie. 1983.

Band 9 Jan Honsel: Das Kaufverhalten im Antiquitätenmarkt. Eine empirische Analyse der Kaufmotive, ihrer Bestimmungsfaktoren und Verhaltenswirkungen. 1984.

\section{SCHRIFTEN ZU MARKETING UND MANAGEMENT}

Band 10 Matthias Krups: Marketing innovativer Dienstleistungen am Beispiel elektronischer Wirtschaftsinformationsdienste. 1985.

Band 11 Bernd Faehsler: Emotionale Grundhaltungen als Einflußfaktoren des Kăuferverhaltens. Eine empirische Analyse der Beziehungen zwischen emotionalen Grundhaltungen und ausgewählten Konsumstrukturen. 1986.

Band 12 Ernst-Otto Thiesing: Strategische Marketingplanung in filialisierten Universalbanken. Integrierte Filial- und Kundengruppenstrategien auf der Grundlage erfolgsbeeinflussender Schlüsselfaktoren. 1986.

Band 13 Rainer Landwehr: Standardisierung der internationalen Werbeplanung. Eine Untersuchung der Prozeßstandardisierung am Beispiel der Werbebudgetierung im Automobilmarkt. 1988.

Band 14 Paul-Josef Patt: Strategische Erfolgsfaktoren im Einzelhandel. Eine empirische Analyse am Beispiel des Bekleidungsfachhandels. 1988. 2. Auflage. 1990.

Band 15 Elisabeth Tolle: Der Einfluß ablenkender Tătigkeiten auf die Werbewirkung. Bestimmungsfaktoren der Art und Höhe von Ablenkungseffekten bei Rundfunkspots. 1988.

Band 16 Hanns Ostmeier: Ökologieorientierte Produktinnovationen. Eine empirische Analyse unter besonderer Berücksichtigung ihrer Erfolgseinschătzung. 1990.

Band 17 Bernd Büker: Qualitătsbeurteilung investiver Dienstleistungen. Operationalisienungsansătze an einem empirischen Beispiel zentraler EDV-Dienste. 1991.

Band 18 Kerstin Ch. Monhemius: Umweltbewußtes Kaufverhalten von Konsumenten. Ein Beitrag zur Operationalisierung, Erklärung und Typologie des Verhaltens in der Kaufsituation. 1993. 
Band 19 Uwe Schürmann: Erfolgsfaktoren der Werbung im Produktlebenszyklus. Ein Beitrag zur Werbewirkungsforschung. 1993.

Band 20 Ralf Birkelbach: Qualitätsmanagement in Dienstleistungscentern. Konzeption und typenspezifische Ausgestaltung unter besonderer Berücksichtigung von Verkehrsflughäfen. 1993.

Band 21 Simone Frömbling. Zielgruppenmarketing im Fremdenverkehr von Regionen. Ein Beitrag zur Marktsegmentierung auf der Grundlage von Werten, Motiven und Einstellungen. 1993.

Band 22 Marcus Poggenpohl: Verbundanalyse im Einzelhandel auf der Grundlage von Kundenkarteninformationen. Eine empirische Untersuchung von Verbundbeziehungen zwischen Abteilungen. 1994.

Band 23 Kai Bauche: Segmentierung von Kundendienstleistungen auf investiven Märkten. Dargestellt am Beispiel von Personal Computern. 1994. 


\section{Cornelia Meurer}

\section{Strategisches internationales Marketing für Dienstleistungen Dargestellt am Beispiel des Management-Consulting}

Frankfurt/M., Berlin, Bern, New York, Paris, Wien, 1993. VI, 360 S., 27 Abb. Marktorientierte Unternehmensführung. Herausgegeben von H. Freter. Bd. 16 ISBN 3-631-46012-0 br. DM 89.--

Auch für Dienstleistungen gewinnen Fragen des internationalen wie des strategischen Marketing zunehmend an Bedeutung. Die Arbeit analysiert systematisch und umfassend die Schnittstellen zwischen dem Dienstleistungs-, dem internationalen und dem strategischen Marketing. Sie konkretisiert dies am Beispiel des Management-Consulting. Im Mittelpunkt der Betrachtung steht die Fundierung sowie die Entwicklung und Ausgestaltung von internationalen Marketingstrategien für Consultingleistungen. Hierbei finden Berücksichtigung: 1. Strategien für das Unternehmen als Gesamtheit, 2. Basisstrategien für einzelne Geschäftseinheiten, 3. Instrumentalstrategien für einzelne Geschäftseinheiten. Die Arbeit stellt einen innovativen Beitrag zur Strukturierung und Analyse eines bislang nicht bearbeiteten Themas dar.

Aus dem Inhalt: Begriffliche und sachliche Grundlagen - Unternehmenspolitik Strategische Analyse und Prognose - Grundsatzstrategien für das Unternehmen als Gesamtheit - Basisstrategien für Geschäftseinheiten - Instrumentalstrategien für Geschäftseinheiten

Die Dissertation wurde von Prof. H. Freter an der Universität Siegen betreut. Die Autorin arbeitet in einer Abteilung für Marketingstrategien einer deutschen GroBbank.

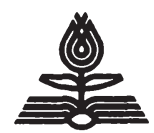

Verlag Peter Lang Frankfurt a.M. Berlin • Bern $\cdot$ New York $\cdot$ Paris $\cdot$ Wien Auslieferung: Verlag Peter Lang AG, Jupiterstr. 15, CH-3000 Bern 15 Telefon (004131) 9411122, Telefax (004131) 9411131

- Preisänderungen vorbehalten - 
Kai Bauche - 978-3-631-75089-6

Downloaded from PubFactory at 01/11/2019 08:30:54AM

via free access 